\title{
Collision Avoidance in Biological Systems using Collision Cones
}

\author{
Beth L Boardman
}

\author{
A thesis \\ submitted in partial fulfillment of the \\ requirements for the degree of

\begin{abstract}
Master of Science in Aeronautics and Astronautics
\end{abstract}
University of Washington

2012

\section{Committee:}

Kristi Morgansen

Juri Vaugners

Program Authorized to Offer Degree:

Aeronautics \& Astronautics 



\section{TABLE OF CONTENTS}

Page

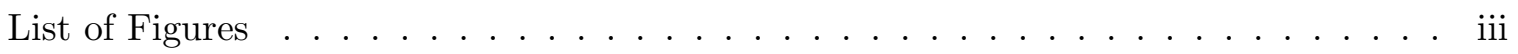

List of Tables . . . . . . . . . . . . . . . . . . . . xviii

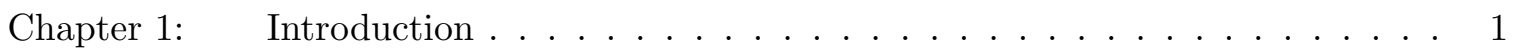

1.1 Engineered Deconfliction . . . . . . . . . . . . . . . . . 1

1.2 Biological Systems . . . . . . . . . . . . . . . . . . . . . . . . . . . . . . .

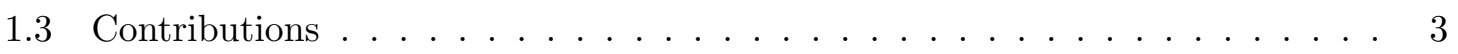

1.4 Outline of Thesis . . . . . . . . . . . . . . . 3

Chapter 2: Biological Species . . . . . . . . . . . . . . . 4

2.1 Giant Danios - Schooling Fish . . . . . . . . . . . . . . . . . . 4

2.2 Barn Swallows - Maneuvering Birds . . . . . . . . . . . . . . . 5

2.3 Cave Bats - Hunting Bats . . . . . . . . . . . . . . . . 7

Chapter 3: Perception and Reaction . . . . . . . . . . . . . 11

3.1 Zonal Model . . . . . . . . . . . . . . . . . . . . . 11

3.2 Number of Influential Nearest Neighbors . . . . . . . . . . . . . . . . . . 13

3.3 Hybrid Criteria: Merging of Maximum Range and Nearest Neighbors . . . . . 14

Chapter 4: Collision Avoidance . . . . . . . . . . . . . . 16

4.1 Coordinate Systems . . . . . . . . . . . . . . . . . 17

4.2 Checking Conflict . . . . . . . . . . . . . . . . . 19

4.3 Moving Out of Conflict . . . . . . . . . . . . . . 20

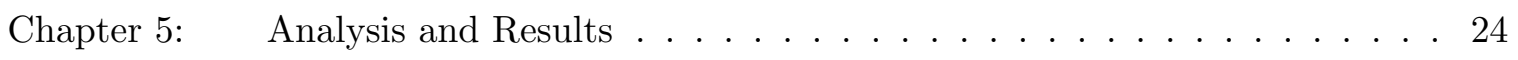

5.1 Metrics and Correlations . . . . . . . . . . . . . . . . . 24

5.2 Giant Danios . . . . . . . . . . . . . . . . . . 25

5.3 Barn Swallows . . . . . . . . . . . . . . . . 66

5.4 Cave Bats . . . . . . . . . . . . . . . . . . . . . 121 
Chapter 6: Conclusion and Future Work . . . . . . . . . . . . . 140

6.1 Future Work . . . . . . . . . . . . . . . . . . . . 141

Bibliography . . . . . . . . . . . . . . . . . . . 142 


\section{LIST OF FIGURES}

Figure Number

Page

2.1 This figure shows the three dimensional position data for the giant danios during the beginning segment of the 6-9 data set. Each colored line represents a trajectory of a different fish. The $x-y$ plane forms the horizontal while the $z$ - direction points antiparallel to gravity. This data segment contains trajectories for 15 giant danios. . . . . . . . . . . . . 5

2.2 The $x-, y-$, and $z$-velocity data for a typical giant danio during the beginnning segment of the 6-9 data set. The topmost plot is the $x$-velocity, the center plot is the $y$-velocity, and the bottom plot is the $z$-velocity. The data segment is one minute long at 30 frames per second. . . . . . . . .

2.3 One of the data sets for position data of barn swallows. The trajectories of 23 barn swallows are shown. Each colored line represents the trajectory of a different bird. The $x-y$ plane is the horizontal plane, while the $z$-direction is antiparallel to gravity. . . . . . . . . . . . . . . .

2.4 This figure plots the different comonents of the velocity data for a typical barn swallow during the flock of 23 birds data set. The topmost plot is the $x$-velocity, the center plot is the $y$-velocity, and the bottom plot is the $z$-velocity. The Flock data set is about 4.5 seconds long at 100 frames per second. . . . . . . . . . . . . . . . .

2.5 This figure shows the three dimensional position data for bats during the data set. A total of 20 bat trajectories form this data set. Each colored line represents a different bat's trajectory. The black lines represent the static obstacles, a vine and a pole. The $x-y$ plane forms the horizontal plane while the $z$-direction is antiparallel to gravity. . . . . . . . . . .

2.6 This figure shows plots of the different components of the velocity data for a typical cave bat during the data set. The topmost plot is the $x$-velocity, the center plot is the $y$-velocity, and the bottom plot is the $z$-velocity. This data set is about four seconds long at 131.5 frames per second. . . . . 10 
3.1 This figure is a depiction of an example of the biological zonal model. The central blue ellipsoid is the biological animal. The green line is the outer boundary of the repulsion zone. The alignment zone's outer boundery is the red line. The outer boundary of the attraction zone is represented by the cyan colored line. The gap in the oval at the bottom of the biological represents the sensing blind spot. Everything outside the attraction zone is ignored. . . . . . . . . . . . . . . . . . 12

4.1 This figure is an example of a global projection. The blue arrow is the original vector, and the green is the vector projected into the horizontal $x-y$ plane. . . . . . . . . . . . . . . . 18

4.2 This figure is an example of a body coordinate projection. The blue arrow represents the original velocity vector and the green vector is the body coordinate velocity vector. The red arrow is the original velocity vector of the obstacle. The cyan colored vector is the body coordinate obstacle velocity vector, and the magenta is that vector projected into the $x_{b}-y_{b}$ plane. . . . . . . . . . . . . . . . . . 2 2

4.3 This figure is typical graphical representation of the constant speed maneuver. The black vector is the current velocity, the green vectory is the next velocity vector, and the red vector is the optimal solution. . . . . . . . . . 21

4.4 This figure is typical graphical representation of the variable speed maneuver. The black vector is the current velocity, the green vectory is the next velocity vector, and the red vector is the optimal solution. . . . . . . . . . 23

5.1 The $\Delta V$ distribution for three influential neighbors and $\delta_{\text {attraction }}=$ infinity for the 0-15 giant danio data set under the global coordinate system with constant speed maneuver. . . . . . . . . . . . . . . 29

5.2 The $\Delta V$ distribution for seven influential neighbors and $\delta_{\text {attraction }}=$ infinity for the 0-15 giant danio data set under the global coordinate system with the constant speed maneuver. . . . . . . . . . . . . . . . . . . 30

5.3 The $\Delta V$ distribution for the maximum number of influential neighbors and $\delta_{\text {attraction }}=$ infinity for the $0-15$ giant danio data set under the global coordinate system with the constant speed maneuver. . . . . . . . . . . . 30

5.4 The $\Delta V$ distribution for three influential neighbors and $\delta_{\text {attraction }}=$ infinity for the 6-9 giant danio data set under the global coordinate system with the constant speed maneuver. . . . . . . . . . . . . . 31

5.5 The $\Delta V$ distribution for seven influential neighbors and $\delta_{\text {attraction }}=$ infinity for the 6-9 giant danio data set under the global coordinate system with the constant speed maneuver. . . . . . . . . . . . . . . . . . 31 
5.6 The $\Delta V$ distribution for the maximum number of influential neighbors and $\delta_{\text {attraction }}=$ infinity for the 6-9 giant danio data set under the global coordinate system with the constant speed maneuver. . . . . . . . . . . . 32

5.7 The $\Delta V$ distribution for three influential neighbors and $\delta_{\text {attraction }}=$ infinity for the 9-6 giant danio data set under the global coordinate system with the constant speed maneuver. . . . . . . . . . . . . . . . . . 32

5.8 The $\Delta V$ distribution for seven influential neighbors and $\delta_{\text {attraction }}=$ infinity for the 9-6 giant danio data set under the global coordinate system with the constant speed maneuver. . . . . . . . . . . . . . . . . . . 33

5.9 The $\Delta V$ distribution for the maximum number of influential neighbors and $\delta_{\text {attraction }}=$ infinity for the 9-6 giant danio data set under the global coordinate system with the constant speed maneuver. . . . . . . . . . . . . 33

5.10 The $\Delta V$ distribution for three influential neighbors and $\delta_{\text {attraction }}=$ infinity for the 12-3 giant danio data set under the global coordinate system with the constant speed maneuver.. . . . . . . . . . . . . . . . . . . 34

5.11 The $\Delta V$ distribution for seven influential neighbors and $\delta_{\text {attraction }}=$ infinity for the 12-3 giant danio data set under the global coordinate system with the constant speed maneuver. . . . . . . . . . . . . . . . . . . 34

5.12 The $\Delta V$ distribution for the maximum number of influential neighbors and $\delta_{\text {attraction }}=$ infinity for the 12-3 giant danio data set under the global coordinate system with the constant speed maneuver. . . . . . . . . . . . 35

5.13 The $\Delta V$ distribution for three influential neighbors and $\delta_{\text {attraction }}=$ infinity for the 15-0 giant danio data set under the global coordinate system with the constant speed maneuver. . . . . . . . . . . . . . . 35

5.14 The $\Delta V$ distribution for seven influential neighbors and $\delta_{\text {attraction }}=$ infinity for the 15-0 giant danio data set under the global coordinate system with the constant speed maneuver. . . . . . . . . . . . . . . . . . 36

5.15 The $\Delta V$ distribution for the maximum number of influential neighbors and $\delta_{\text {attraction }}=$ infinity for the 15 -0 giant danio data set under the global coordinate system with the constant speed maneuver. . . . . . . . . . . 36

5.16 The $\Delta V$ distribution for three influential neighbors and $\delta_{\text {attraction }}=$ infinity for the 0-15 giant danio data set under the global coordinate system with the variable speed maneuver. . . . . . . . . . . . . . . . . 37

5.17 The $\Delta V$ distribution for seven influential neighbors and $\delta_{\text {attraction }}=$ infinity for the 0-15 giant danio data set under the global coordinate system with the variable speed maneuver. . . . . . . . . . . . . . . 38

5.18 The $\Delta V$ distribution for the maximum number of influential neighbors and $\delta_{\text {attraction }}=$ infinity for the $0-15$ giant danio data set under the global coordinate system with the variable speed maneuver. . . . . . . . . . . 38 
5.19 The $\Delta V$ distribution for three influential neighbors and $\delta_{\text {attraction }}=$ infinity for the 6-9 giant danio data set under the global coordinate system with the variable speed maneuver. . . . . . . . . . . . . . . . . . 39

5.20 The $\Delta V$ distribution for seven influential neighbors and $\delta_{\text {attraction }}=$ infinity for the 6-9 giant danio data set under the global coordinate system with the variable speed maneuver. . . . . . . . . . . . . . . . . . . . . 39

5.21 The $\Delta V$ distribution forthe maximum number of influential neighbors and $\delta_{\text {attraction }}=$ infinity for the 6-9 giant danio data set under the global coordinate system with the variable speed maneuver. . . . . . . . . . . . . 40

5.22 The $\Delta V$ distribution for three influential neighbors and $\delta_{\text {attraction }}=$ infinity for the 9-6 giant danio data set under the global coordinate system with the variable speed maneuver. . . . . . . . . . . . . . . . . . 40

5.23 The $\Delta V$ distribution for seven influential neighbors and $\delta_{\text {attraction }}=$ infinity for the 9-6 giant danio data set under the global coordinate system with the variable speed maneuver. . . . . . . . . . . . . . . . . . . 4

5.24 The $\Delta V$ distribution for the maximum number of influential neighbors and $\delta_{\text {attraction }}=$ infinity for the 9-6 giant danio data set under the global coordinate system with the variable speed maneuver. . . . . . . . . . . . . . 4

5.25 The $\Delta V$ distribution for three influential neighbors and $\delta_{\text {attraction }}=$ infinity for the 12-3 giant danio data set under the global coordinate system with the variable speed maneuver. . . . . . . . . . . . . . . . . . . 42

5.26 The $\Delta V$ distribution for seven influential neighbors and $\delta_{\text {attraction }}=$ infinity for the 12-3 giant danio data set under the global coordinate system with the variable speed maneuver. . . . . . . . . . . . . . . . . 42

5.27 The $\Delta V$ distribution for the maximum number of influential neighbors and $\delta_{\text {attraction }}=$ infinity for the 12-3 giant danio data set under the global coordinate system with the variable speed maneuver. . . . . . . . . . . . 43

5.28 The $\Delta V$ distribution for seven influential neighbors and $\delta_{\text {attraction }}=20 \mathrm{~cm}$ for the 12-3 giant danio data set under the global coordinate system with the variable speed maneuver. . . . . . . . . . . . . . . . . . 43

5.29 The $\Delta V$ distribution for seven influential neighbors and $\delta_{\text {attraction }}=15 \mathrm{~cm}$ for the 12-3 giant danio data set under the global coordinate system with the variable speed maneuver. . . . . . . . . . . . . . . . . . . . 44

5.30 The $\Delta V$ distribution for seven influential neighbors and $\delta_{\text {attraction }}=10 \mathrm{~cm}$ for the 12-3 giant danio data set under the global coordinate system with the variable speed maneuver. . . . . . . . . . . . . . . . . . . . . . 44

5.31 The $\Delta V$ distribution for three influential neighbors and $\delta_{\text {attraction }}=$ infinity for the 15-0 giant danio data set under the global coordinate system with the variable speed maneuver. . . . . . . . . . . . . . . . . 45 
5.32 The $\Delta V$ distribution for seven influential neighbors and $\delta_{\text {attraction }}=$ infinity for the 15-0 giant danio data set under the global coordinate system with the variable speed maneuver. . . . . . . . . . . . . . . . . 45

5.33 The $\Delta V$ distribution for the maximum number of influential neighbors and $\delta_{\text {attraction }}=$ infinity for the 15 -0 giant danio data set under the global coordinate system with the variable speed maneuver.. . . . . . . . . . . . . 46

5.34 The $\Delta V$ distribution for seven influential neighbors and $\delta_{\text {attraction }}=20 \mathrm{~cm}$ for the 15-0 giant danio data set under the global coordinate system with the variable speed maneuver. . . . . . . . . . . . . . . . . 46

5.35 The $\Delta V$ distribution for seven influential neighbors and $\delta_{\text {attraction }}=15 \mathrm{~cm}$ for the 15-0 giant danio data set under the global coordinate system with the variable speed maneuver. . . . . . . . . . . . . . . . . . . 47

5.36 The $\Delta V$ distribution for seven influential neighbors and $\delta_{\text {attraction }}=10 \mathrm{~cm}$ for the 15-0 giant danio data set under the global coordinate system with the variable speed maneuver. . . . . . . . . . . . . . . . . . . 47

5.37 The $\Delta V$ distribution for three influential neighbors and $\delta_{\text {attraction }}=$ infinity for the 0-15 giant danio data set under the body-fixed coordinate system with the constant speed maneuver. . . . . . . . . . . . . . . . . . . . 48

5.38 The $\Delta V$ distribution for seven influential neighbors and $\delta_{\text {attraction }}=$ infinity for the 0-15 giant danio data set under the body-fixed coordinate system with the constant speed maneuver. . . . . . . . . . . . . . . . .

5.39 The $\Delta V$ distribution for maximum number of influential neighbors and $\delta_{\text {attraction }}=$ infinity for the $0-15$ giant danio data set under the body-fixed coordinate system with the constant speed maneuver. . . . . . . . . . . 49

5.40 The $\Delta V$ distribution for three influential neighbors and $\delta_{\text {attraction }}=$ infinity for the 6-9 giant danio data set under the body-fixed coordinate system with the constant speed maneuver. . . . . . . . . . . . . . . 50

5.41 The $\Delta V$ distribution for seven influential neighbors and $\delta_{\text {attraction }}=$ infinity for the 6-9 giant danio data set under the body-fixed coordinate system with the constant speed maneuver. . . . . . . . . . . . . . . 50

5.42 The $\Delta V$ distribution for the maximum number of influential neighbors and $\delta_{\text {attraction }}=$ infinity for the 6-9 giant danio data set under the body-fixed coordinate system with the constant speed maneuver. . . . . . . . . . 51

5.43 The $\Delta V$ distribution for three influential neighbors and $\delta_{\text {attraction }}=$ infinity for the 9-6 giant danio data set under the body-fixed coordinate system with the constant speed maneuver. . . . . . . . . . . . . . . . 51

5.44 The $\Delta V$ distribution for seven influential neighbors and $\delta_{\text {attraction }}=$ infinity for the 9-6 giant danio data set under the body-fixed coordinate system with the constant speed maneuver. . . . . . . . . . . . . . . . 52 
5.45 The $\Delta V$ distribution for the maximum number of influential neighbors and $\delta_{\text {attraction }}=$ infinity for the 9-6 giant danio data set under the body-fixed coordinate system with the constant speed maneuver. . . . . . . . . . . 52

5.46 The $\Delta V$ distribution for three influential neighbors and $\delta_{\text {attraction }}=$ infinity for the 12-3 giant danio data set under the body-fixed coordinate system with the constant speed maneuver. . . . . . . . . . . . . . . 53

5.47 The $\Delta V$ distribution for seven influential neighbors and $\delta_{\text {attraction }}=$ infinity for the 12-3 giant danio data set under the body-fixed coordinate system with the constant speed maneuver. . . . . . . . . . . . . 53

5.48 The $\Delta V$ distribution for the maximum number of influential neighbors and $\delta_{\text {attraction }}=$ infinity for the 12-3 giant danio data set under the body-fixed coordinate system with the constant speed maneuver. . . . . . . . . . . 54

5.49 The $\Delta V$ distribution for three influential neighbors and $\delta_{\text {attraction }}=$ infinity for the 15-0 giant danio data set under the body-fixed coordinate system with the constant speed maneuver. . . . . . . . . . . . . . 54

5.50 The $\Delta V$ distribution for seven influential neighbors and $\delta_{\text {attraction }}=$ infinity for the 15-0 giant danio data set under the body-fixed coordinate system with the constant speed maneuver. . . . . . . . . . . . . . 55

5.51 The $\Delta V$ distribution for the maximum number of influential neighbors and $\delta_{\text {attraction }}=$ infinity for the $15-0$ giant danio data set under the body-fixed coordinate system with the constant speed maneuver. . . . . . . . . 55

5.52 The $\Delta V$ distribution for the maximum number of influential neighbors and $\delta_{\text {attraction }}=20 \mathrm{~cm}$ for the 15-0 giant danio data set under the body-fixed coordinate system with the constant speed maneuver. . . . . . . . . 56

5.53 The $\Delta V$ distribution for the maximum number of influential neighbors and $\delta_{\text {attraction }}=15 \mathrm{~cm}$ for the 15-0 giant danio data set under the body-fixed coordinate system with the constant speed maneuver. . . . . . . . . 56

5.54 The $\Delta V$ distribution for the maximum number of influential neighbors and $\delta_{\text {attraction }}=10 \mathrm{~cm}$ for the $15-0$ giant danio data set under the body-fixed coordinate system with the constant speed maneuver. . . . . . . . . . 57

5.55 The $\Delta V$ distribution for three influential neighbors and $\delta_{\text {attraction }}=$ infinity for the 0-15 giant danio data set under the body-fixed coordinate system with the variable speed maneuver. . . . . . . . . . . . . 58

5.56 The $\Delta V$ distribution for seven influential neighbors and $\delta_{\text {attraction }}=$ infinity for the 0-15 giant danio data set under the body-fixed coordinate system with the variable speed maneuver. . . . . . . . . . . . . . . 59

5.57 The $\Delta V$ distribution for the maximum number of influential neighbors and $\delta_{\text {attraction }}=$ infinity for the $0-15$ giant danio data set under the body-fixed coordinate system with the variable speed maneuver. . . . . . . . . . . 59 
5.58 The $\Delta V$ distribution for three influential neighbors and $\delta_{\text {attraction }}=$ infinity for the 6-9 giant danio data set under the body-fixed coordinate system with the variable speed maneuver. . . . . . . . . . . . . . . . 60

5.59 The $\Delta V$ distribution for seven influential neighbors and $\delta_{\text {attraction }}=$ infinity for the 6-9 giant danio data set under the body-fixed coordinate system with the variable speed maneuver. . . . . . . . . . . . . . . . . . 60

5.60 The $\Delta V$ distribution for the maximum number of influential neighbors and $\delta_{\text {attraction }}=$ infinity for the 6-9 giant danio data set under the body-fixed coordinate system with the variable speed maneuver. . . . . . . . . . . . 61

5.61 The $\Delta V$ distribution for three influential neighbors and $\delta_{\text {attraction }}=$ infinity for the 9-6 giant danio data set under the body-fixed coordinate system with the variable speed maneuver. . . . . . . . . . . . . . 61

5.62 The $\Delta V$ distribution for seven influential neighbors and $\delta_{\text {attraction }}=$ infinity for the 9-6 giant danio data set under the body-fixed coordinate system with the variable speed maneuver. . . . . . . . . . . . . . . 62

5.63 The $\Delta V$ distribution for the maximum number of influential neighbors and $\delta_{\text {attraction }}=$ infinity for the 9-6 giant danio data set under the body-fixed coordinate system with the variable speed maneuver. . . . . . . . . . . . . 62

5.64 The $\Delta V$ distribution for three influential neighbors and $\delta_{\text {attraction }}=$ infinity for the 12-3 giant danio data set under the body-fixed coordinate system with the variable speed maneuver. . . . . . . . . . . . . . 63

5.65 The $\Delta V$ distribution for seven influential neighbors and $\delta_{\text {attraction }}=$ infinity for the 12-3 giant danio data set under the body-fixed coordinate system with the variable speed maneuver. . . . . . . . . . . . . . 63

5.66 The $\Delta V$ distribution for the maximum number of influential neighbors and $\delta_{\text {attraction }}=$ infinity for the 12-3 giant danio data set under the body-fixed coordinate system with the variable speed maneuver. . . . . . . . . . . . 64

5.67 The $\Delta V$ distribution for three influential neighbors and $\delta_{\text {attraction }}=$ infinity for the 15-0 giant danio data set under the body-fixed coordinate system with the variable speed maneuver. . . . . . . . . . . . . . . . 64

5.68 The $\Delta V$ distribution for seven influential neighbors and $\delta_{\text {attraction }}=$ infinity for the 15-0 giant danio data set under the body-fixed coordinate system with the variable speed maneuver. . . . . . . . . . . . . 65

5.69 The $\Delta V$ distribution for the maximum number of influential neighbors and $\delta_{\text {attraction }}=$ infinity for the 15-0 giant danio data set under the body-fixed coordinate system with the variable speed maneuver. . . . . . . . . 65

5.70 The trajectory data for Trial03-2012 showing the two barn swallows and the truck. The stars mark the beginning of the trajectories. . . . . . . . . 67 
5.71 The $\Delta V$ distribution for three influential neighbors and $\delta_{\text {attraction }}=$ infinity for the Flock barn swallow data set under the global coordinate system with the constant speed maneuver. . . . . . . . . . . . . . . . . 72

5.72 The $\Delta V$ distribution for seven influential neighbors and $\delta_{\text {attraction }}=$ infinity for the Flock barn swallow data set under the global coordinate system with the constant speed maneuver. . . . . . . . . . . . . . . . . . 73

5.73 The $\Delta V$ distribution for the maximum number of influential neighbors and $\delta_{\text {attraction }}=$ infinity for the Flock barn swallow data set under the global coordinate system with the constant speed maneuver. . . . . . . . . . 73

5.74 The $\Delta V$ distribution for seven influential neighbors and $\delta_{\text {attraction }}=5 \mathrm{~m}$ for the Flock barn swallow data set under the global coordinate system with the constant speed maneuver. . . . . . . . . . . . . . . . . . . . . 74

5.75 The $\Delta V$ distribution for seven influential neighbors and $\delta_{\text {attraction }}=3 \mathrm{~m}$ for the Flock barn swallow data set under the global coordinate system with the constant speed maneuver. . . . . . . . . . . . . . . . . . . . . . 74

5.76 The $\Delta V$ distribution for seven influential neighbors and $\delta_{\text {attraction }}=1.5 \mathrm{~m}$ for the Flock barn swallow data set under the global coordinate system with the constant speed maneuver. . . . . . . . . . . . . . . . . . . . 75

5.77 The $\Delta V$ distribution for three influential neighbors and $\delta_{\text {attraction }}=$ infinity for the Trial03 barn swallow data set under the global coordinate system with the constant speed maneuver. . . . . . . . . . . . . . . . 75

5.78 The $\Delta V$ distribution for seven influential neighbors and $\delta_{\text {attraction }}=$ infinity for the Trial03 barn swallow data set under the global coordinate system with the constant speed maneuver. . . . . . . . . . . . . . . 76

5.79 The $\Delta V$ distribution for the maximum number of influential neighbors and $\delta_{\text {attraction }}=$ infinity for the Trial03 barn swallow data set under the global coordinate system with the constant speed maneuver. . . . . . . . . 76

5.80 The $\Delta V$ distribution for seven influential neighbors and $\delta_{\text {attraction }}=5 \mathrm{~m}$ for the Trial03 barn swallow data set under the global coordinate system with the constant speed maneuver. . . . . . . . . . . . . . . . . . 77

5.81 The $\Delta V$ distribution for seven influential neighbors and $\delta_{\text {attraction }}=3 \mathrm{~m}$ for the Trial03 barn swallow data set under the global coordinate system with the constant speed maneuver. . . . . . . . . . . . . . . . . . 77

5.82 The $\Delta V$ distribution for seven influential neighbors and $\delta_{\text {attraction }}=1.5 \mathrm{~m}$ for the Trial03 barn swallow data set under the global coordinate system with the constant speed maneuver. . . . . . . . . . . . 78

5.83 The $\Delta V$ distribution for three influential neighbors and $\delta_{\text {attraction }}=$ infinity for the Trial04 barn swallow data set under the global coordinate system with the constant speed maneuver. . . . . . . . . . . . . . . . . . 79 
5.84 The $\Delta V$ distribution for three influential neighbors and $\delta_{\text {attraction }}=5 \mathrm{~m}$ for the Trial04 barn swallow data set under the global coordinate system with the constant speed maneuver. . . . . . . . . . . . . . . . . 80

5.85 The $\Delta V$ distribution for three influential neighbors and $\delta_{\text {attraction }}=3 \mathrm{~m}$ for the Trial04 barn swallow data set under the global coordinate system with the constant speed maneuver. . . . . . . . . . . . . . . . . . . . 80

5.86 The $\Delta V$ distribution for three influential neighbors and $\delta_{\text {attraction }}=1.5 \mathrm{~m}$ for the Trial04 barn swallow data set under the global coordinate system with the constant speed maneuver. . . . . . . . . . . . . . . . . . 81

5.87 The $\Delta V$ distribution for three influential neighbors and $\delta_{\text {attraction }}=$ infinity for the Trial02-2012 barn swallow data set under the global coordinate system with the constant speed maneuver. . . . . . . . . . . . . 82

5.88 The $\Delta V$ distribution for three influential neighbors and $\delta_{\text {attraction }}=5 \mathrm{~m}$ for the Trial02-2012 barn swallow data set under the global coordinate system with the constant speed maneuver. . . . . . . . . . . . . . . . . 83

5.89 The $\Delta V$ distribution for three influential neighbors and $\delta_{\text {attraction }}=3 \mathrm{~m}$ for the Trial02-2012 barn swallow data set under the global coordinate system with the constant speed maneuver. . . . . . . . . . . . . . . 83

5.90 The $\Delta V$ distribution for three influential neighbors and $\delta_{\text {attraction }}=1.5 \mathrm{~m}$ for the Trial02-2012 barn swallow data set under the global coordinate system with the constant speed maneuver. . . . . . . . . . . . . . . . .

5.91 The $\Delta V$ distribution for three influential neighbors and $\delta_{\text {attraction }}=$ infinity for the Flock barn swallow data set under the global coordinate system with the variable speed maneuver. . . . . . . . . . . . . . 85

5.92 The $\Delta V$ distribution for seven influential neighbors and $\delta_{\text {attraction }}=$ infinity for the Flock barn swallow data set under the global coordinate system with the variable speed maneuver. . . . . . . . . . . . . 86

5.93 The $\Delta V$ distribution for the maximum number of influential neighbors and $\delta_{\text {attraction }}=$ infinity for the Flock barn swallow data set under the global coordinate system with the variable speed maneuver. . . . . . . . . 86

5.94 The $\Delta V$ distribution for seven influential neighbors and $\delta_{\text {attraction }}=5 \mathrm{~m}$ for the Flock barn swallow data set under the global coordinate system with the variable speed maneuver. . . . . . . . . . . . . . . . . 87

5.95 The $\Delta V$ distribution for seven influential neighbors and $\delta_{\text {attraction }}=3 \mathrm{~m}$ for the Flock barn swallow data set under the global coordinate system with the variable speed maneuver. . . . . . . . . . . . . . . . . . . 87

5.96 The $\Delta V$ distribution for seven influential neighbors and $\delta_{\text {attraction }}=1.5$ for the Flock barn swallow data set under the global coordinate system with the variable speed maneuver. . . . . . . . . . . . . . . . 88 
5.97 The $\Delta V$ distribution for three influential neighbors and $\delta_{\text {attraction }}=$ infinity for the Trial03 barn swallow data set under the global coordinate system with the variable speed maneuver. . . . . . . . . . . . . . . 89

5.98 The $\Delta V$ distribution for seven influential neighbors and $\delta_{\text {attraction }}=$ infinity for the Trial03 barn swallow data set under the global coordinate system with the variable speed maneuver. . . . . . . . . . . . . . . . . 90

5.99 The $\Delta V$ distribution for the maximum number of influential neighbors and $\delta_{\text {attraction }}=$ infinity for the Trial03 barn swallow data set under the global coordinate system with the variable speed maneuver. . . . . . . . . . 90

5.100 The $\Delta V$ distribution for seven influential neighbors and $\delta_{\text {attraction }}=5 \mathrm{~m}$ for the Trial03 barn swallow data set under the global coordinate system with the variable speed maneuver. . . . . . . . . . . . . . . . . . . 91

5.101 The $\Delta V$ distribution for seven influential neighbors and $\delta_{\text {attraction }}=3 \mathrm{~m}$ for the Trial03 barn swallow data set under the global coordinate system with the variable speed maneuver. . . . . . . . . . . . . . . . . 91

5.102 The $\Delta V$ distribution for seven influential neighbors and $\delta_{\text {attraction }}=1.5 \mathrm{~m}$ for the Trial03 barn swallow data set under the global coordinate system with the variable speed maneuver. . . . . . . . . . . . . . . . . 92

5.103 The $\Delta V$ distribution for three influential neighbors and $\delta_{\text {attraction }}=$ infinity for the Trial04 barn swallow data set under the global coordinate system with the variable speed maneuver. . . . . . . . . . . . . . . 93

5.104 The $\Delta V$ distribution for three influential neighbors and $\delta_{\text {attraction }}=5 \mathrm{~m}$ for the Trial04 barn swallow data set under the global coordinate system with the variable speed maneuver. . . . . . . . . . . . . . . . . . . 94

5.105 The $\Delta V$ distribution for three influential neighbors and $\delta_{\text {attraction }}=3 \mathrm{~m}$ for the Trial04 barn swallow data set under the global coordinate system with the variable speed maneuver. . . . . . . . . . . . . . . . . . 94

5.106 The $\Delta V$ distribution for three influential neighbors and $\delta_{\text {attraction }}=1.5 \mathrm{~m}$ for the Trial04 barn swallow data set under the global coordinate system with the variable speed maneuver. . . . . . . . . . . . . . 95

5.107 The $\Delta V$ distribution for three influential neighbors and $\delta_{\text {attraction }}=$ infinity for the Trial02-2012 barn swallow data set under the global coordinate system with the variable speed maneuver. . . . . . . . . . . 96

5.108 The $\Delta V$ distribution for three influential neighbors and $\delta_{\text {attraction }}=5 \mathrm{~m}$ for the Trial02-2012 barn swallow data set under the global coordinate system with the variable speed maneuver. . . . . . . . . . . . . . . 97

5.109 The $\Delta V$ distribution for three influential neighbors and $\delta_{\text {attraction }}=3 \mathrm{~m}$ for the Trial02-2012 barn swallow data set under the global coordinate system with the variable speed maneuver. . . . . . . . . . . . . . . 97 
5.110 The $\Delta V$ distribution for three influential neighbors and $\delta_{\text {attraction }}=1.5 \mathrm{~m}$ for the Trial02-2012 barn swallow data set under the global coordinate system with the variable speed maneuver. . . . . . . . . . . . . . . . 98

5.111 The $\Delta V$ distribution for three influential neighbors and $\delta_{\text {attraction }}=$ infinity for the Flock barn swallow data set under the body-fixed coordinate system with the constant speed maneuver. . . . . . . . . . . . . . . . 99

5.112 The $\Delta V$ distribution for seven influential neighbors and $\delta_{\text {attraction }}=$ infinity for the Flock barn swallow data set under the body-fixed coordinate system with the constant speed maneuver. . . . . . . . . . . . . . 100

5.113 The $\Delta V$ distribution for the maximum number of influential neighbors and $\delta_{\text {attraction }}=$ infinity for the Flock barn swallow data set under the body-fixed coordinate system with the constant speed maneuver. . . . . . . . . . . 100

5.114 The $\Delta V$ distribution for seven influential neighbors and $\delta_{\text {attraction }}=5 \mathrm{~m}$ for the Flock barn swallow data set under the body-fixed coordinate system with the constant speed maneuver. . . . . . . . . . . . . . . . . 101

5.115 The $\Delta V$ distribution for seven influential neighbors and $\delta_{\text {attraction }}=3 \mathrm{~m}$ for the Flock barn swallow data set under the body-fixed coordinate system with the constant speed maneuver. . . . . . . . . . . . . . . . . . . . . 101

5.116 The $\Delta V$ distribution for seven influential neighbors and $\delta_{\text {attraction }}=1.5 \mathrm{~m}$ for the Flock barn swallow data set under the body-fixed coordinate system with the constant speed maneuver. . . . . . . . . . . . . . . 102

5.117 The $\Delta V$ distribution for three influential neighbors and $\delta_{\text {attraction }}=$ infinity for the Trial03 barn swallow data set under the body-fixed coordinate system with the constant speed maneuver. . . . . . . . . . . . . 103

5.118 The $\Delta V$ distribution for seven influential neighbors and $\delta_{\text {attraction }}=$ infinity for the Trial03 barn swallow data set under the body-fixed coordinate system with the constant speed maneuver. . . . . . . . . . . . . . 104

5.119 The $\Delta V$ distribution for the maximum number of influential neighbors and $\delta_{\text {attraction }}=$ infinity for the Trial03 barn swallow data set under the bodyfixed coordinate system with the constant speed maneuver. . . . . . . . . 104

5.120 The $\Delta V$ distribution for seven influential neighbors and $\delta_{\text {attraction }}=5 \mathrm{~m}$ for the Trial03 barn swallow data set under the body-fixed coordinate system with the constant speed maneuver. . . . . . . . . . . . . . . 105

5.121 The $\Delta V$ distribution for seven influential neighbors and $\delta_{\text {attraction }}=3 \mathrm{~m}$ for the Trial03 barn swallow data set under the body-fixed coordinate system with the constant speed maneuver. . . . . . . . . . . . . . . 105

5.122 The $\Delta V$ distribution for seven influential neighbors and $\delta_{\text {attraction }}=1.5 \mathrm{~m}$ for the Trial03 barn swallow data set under the body-fixed coordinate system with the constant speed maneuver. . . . . . . . . . . . . . . . 106 
5.123 The $\Delta V$ distribution for three influential neighbors and $\delta_{\text {attraction }}=$ infinity for the Trial04 barn swallow data set under the body-fixed coordinate system with the constant speed maneuver. . . . . . . . . . . 106

5.124 The $\Delta V$ distribution for three influential neighbors and $\delta_{\text {attraction }}=5 \mathrm{~m}$ for the Trial04 barn swallow data set under the body-fixed coordinate system with the constant speed maneuver. . . . . . . . . . . . . . . . . 107

5.125 The $\Delta V$ distribution for three influential neighbors and $\delta_{\text {attraction }}=3 \mathrm{~m}$ for the Trial04 barn swallow data set under the body-fixed coordinate system with the constant speed maneuver. . . . . . . . . . . . . . . . 107

5.126 The $\Delta V$ distribution for three influential neighbors and $\delta_{\text {attraction }}=1.5 \mathrm{~m}$ for the Trial04 barn swallow data set under the body-fixed coordinate system with the constant speed maneuver. . . . . . . . . . . . . . . . . . 108

5.127 The $\Delta V$ distribution for three influential neighbors and $\delta_{\text {attraction }}=$ infinity for the Trial02-2012 barn swallow data set under the body-fixed coordinate system with the constant speed maneuver. . . . . . . . . . . . 108

5.128 The $\Delta V$ distribution for three influential neighbors and $\delta_{\text {attraction }}=5 \mathrm{~m}$ for the Trial02-2012 barn swallow data set under the body-fixed coordinate system with the constant speed maneuver. . . . . . . . . . . . . . 109

5.129 The $\Delta V$ distribution for three influential neighbors and $\delta_{\text {attraction }}=3 \mathrm{~m}$ for the Trial02-2012 barn swallow data set under the body-fixed coordinate system with the constant speed maneuver. . . . . . . . . . . . . . . 109

5.130 The $\Delta V$ distribution for three influential neighbors and $\delta_{\text {attraction }}=1.5 \mathrm{~m}$ for the Trial02-2012 barn swallow data set under the body-fixed coordinate system with the constant speed maneuver. . . . . . . . . . . . . . . . 110

5.131 The $\Delta V$ distribution for three influential neighbors and $\delta_{\text {attraction }}=$ infinity for the Flock barn swallow data set under the body-fixed coordinate system with the variable speed maneuver. . . . . . . . . . . . . . . . 110

5.132 The $\Delta V$ distribution for seven influential neighbors and $\delta_{\text {attraction }}=$ infinity for the Flock barn swallow data set under the body-fixed coordinate system with the variable speed maneuver. . . . . . . . . . . . . . . . 111

5.133 The $\Delta V$ distribution for the maximum number of influential neighbors and $\delta_{\text {attraction }}=$ infinity for the Flock barn swallow data set under the body-fixed coordinate system with the variable speed maneuver. . . . . . . . . . . 111

5.134 The $\Delta V$ distribution for seven influential neighbors and $\delta_{\text {attraction }}=5 \mathrm{~m}$ for the Flock barn swallow data set under the body-fixed coordinate system with the variable speed maneuver. . . . . . . . . . . . . . . . . . 112

5.135 The $\Delta V$ distribution for seven influential neighbors and $\delta_{\text {attraction }}=3 \mathrm{~m}$ for the Flock barn swallow data set under the body-fixed coordinate system with the variable speed maneuver. . . . . . . . . . . . . . . . . 112 
5.136 The $\Delta V$ distribution for seven influential neighbors and $\delta_{\text {attraction }}=1.5 \mathrm{~m}$ for the Flock barn swallow data set under the body-fixed coordinate system with the variable speed maneuver. . . . . . . . . . . . . . . . . 113

5.137 The $\Delta V$ distribution for three influential neighbors and $\delta_{\text {attraction }}=$ infinity for the Trial03 barn swallow data set under the body-fixed coordinate system with the variable speed maneuver. . . . . . . . . . . . . . . 113

5.138 The $\Delta V$ distribution for seven influential neighbors and $\delta_{\text {attraction }}=$ infinity for the Trial03 barn swallow data set under the body-fixed coordinate system with the variable speed maneuver. . . . . . . . . . . . . . . 114

5.139 The $\Delta V$ distribution for the maximum number of influential neighbors and $\delta_{\text {attraction }}=$ infinity for the Trial03 barn swallow data set under the bodyfixed coordinate system with the variable speed maneuver. . . . . . . . . . . 114

5.140 The $\Delta V$ distribution for seven influential neighbors and $\delta_{\text {attraction }}=5 \mathrm{~m}$ for the Trial03 barn swallow data set under the body-fixed coordinate system with the variable speed maneuver. . . . . . . . . . . . . . . . . . . 115

5.141 The $\Delta V$ distribution for seven influential neighbors and $\delta_{\text {attraction }}=3 \mathrm{~m}$ for the Trial03 barn swallow data set under the body-fixed coordinate system with the variable speed maneuver. . . . . . . . . . . . . . . . . 115

5.142 The $\Delta V$ distribution for seven influential neighbors and $\delta_{\text {attraction }}=1.5 \mathrm{~m}$ for the Trial03 barn swallow data set under the body-fixed coordinate system with the variable speed maneuver. . . . . . . . . . . . . . . . . 116

5.143 The $\Delta V$ distribution for three influential neighbors and $\delta_{\text {attraction }}=$ infinity for the Trial04 barn swallow data set under the body-fixed coordinate system with the variable speed maneuver. . . . . . . . . . . . 116

5.144 The $\Delta V$ distribution for three influential neighbors and $\delta_{\text {attraction }}=5 \mathrm{~m}$ for the Trial04 barn swallow data set under the body-fixed coordinate system with the variable speed maneuver. . . . . . . . . . . . . . . 117

5.145 The $\Delta V$ distribution for three influential neighbors and $\delta_{\text {attraction }}=3 \mathrm{~m}$ for the Trial04 barn swallow data set under the body-fixed coordinate system with the variable speed maneuver. . . . . . . . . . . . . . . 117

5.146 The $\Delta V$ distribution for three influential neighbors and $\delta_{\text {attraction }}=1.5 \mathrm{~m}$ for the Trial04 barn swallow data set under the body-fixed coordinate system with the variable speed maneuver. . . . . . . . . . . . . . . 118

5.147 The $\Delta V$ distribution for three influential neighbors and $\delta_{\text {attraction }}=$ infinity for the Trial02-2012 barn swallow data set under the body-fixed coordinate system with the variable speed maneuver. . . . . . . . . . . 118

5.148 The $\Delta V$ distribution for three influential neighbors and $\delta_{\text {attraction }}=5 \mathrm{~m}$ for the Trial02-2012 barn swallow data set under the body-fixed coordinate system with the variable speed maneuver. . . . . . . . . . . . . . . . 119 
5.149 The $\Delta V$ distribution for three influential neighbors and $\delta_{\text {attraction }}=3 \mathrm{~m}$ for the Trial02-2012 barn swallow data set under the body-fixed coordinate system with the variable speed maneuver. . . . . . . . . . . . . . . 119

5.150 The $\Delta V$ distribution for three influential neighbors and $\delta_{\text {attraction }}=1.5 \mathrm{~m}$ for the Trial02-2012 barn swallow data set under the body-fixed coordinate system with the variable speed maneuver. . . . . . . . . . . . . . . . . 120

5.151 The $\Delta V$ distribution for three influential neighbors and $\delta_{\text {attraction }}=$ infinity for the bat data set under the global coordinate system with the constant speed maneuver. . . . . . . . . . . . . . . . . . . . . 125

5.152 The $\Delta V$ distribution for five influential neighbors and $\delta_{\text {attraction }}=$ infinity for the bat data set under the global coordinate system with the constant speed maneuver. . . . . . . . . . . . . . . . . . . 125

5.153 The $\Delta V$ distribution for seven influential neighbors and $\delta_{\text {attraction }}=$ infinity for the bat data set under the global coordinate system with the constant speed maneuver. . . . . . . . . . . . . . . . . 126

5.154 The $\Delta V$ distribution for the maximum number of influential neighbors and $\delta_{\text {attraction }}=$ infinity for the bat data set under the global coordinate system with the constant speed maneuver. . . . . . . . . . . . . 126

5.155 The $\Delta V$ distribution for three influential neighbors and $\delta_{\text {attraction }}=$ infinity for the bat data set under the global coordinate system with the variable speed maneuver. . . . . . . . . . . . . . . . . . . . . . 128

5.156 The $\Delta V$ distribution for five influential neighbors and $\delta_{\text {attraction }}=$ infinity for the bat data set under the global coordinate system with the variable speed maneuver. . . . . . . . . . . . . . . . . . . . . 128

5.157 The $\Delta V$ distribution for seven influential neighbors and $\delta_{\text {attraction }}=$ infinity for the bat data set under the global coordinate system with the variable speed maneuver. . . . . . . . . . . . . . . . . 12

5.158 The $\Delta V$ distribution for the maximum number of influential neighbors and $\delta_{\text {attraction }}=$ infinity for the bat data set under the global coordinate system with the variable speed maneuver. . . . . . . . . . . . . . . . . . . 129

5.159 The $\Delta V$ distribution for three influential neighbors and $\delta_{\text {attraction }}=$ infinity for the bat data set under the body-fixed coordinate system with the constant speed maneuver. . . . . . . . . . . . . . . . . . . 130

5.160 The $\Delta V$ distribution for five influential neighbors and $\delta_{\text {attraction }}=$ infinity for the bat data set under the body-fixed coordinate system with the constant speed maneuver. . . . . . . . . . . . . . . . . . . 131

5.161 The $\Delta V$ distribution for seven influential neighbors and $\delta_{\text {attraction }}=$ infinity for the bat data set under the body-fixed coordinate system with the constant speed maneuver. . . . . . . . . . . . . . . . . . 131 
5.162 The $\Delta V$ distribution for the maximum number of influential neighbors and $\delta_{\text {attraction }}=$ infinity for the bat data set under the body-fixed coordinate system with the constant speed maneuver. . . . . . . . . . . . . . . 132

5.163 The $\Delta V$ distribution for the maximum number of influential neighbors and $\delta_{\text {attraction }}=3 \mathrm{~m}$ for the bat data set under the body-fixed coordinate system with the constant speed maneuver. . . . . . . . . . . . . . . . . . . 132

5.164 The $\Delta V$ distribution for the maximum number of influential neighbors and $\delta_{\text {attraction }}=1 \mathrm{~m}$ for the bat data set under the body-fixed coordinate system with the constant speed maneuver. . . . . . . . . . . . . . . . . . 133

5.165 The $\Delta V$ distribution for the maximum number of influential neighbors and $\delta_{\text {attraction }}=0.5 \mathrm{~m}$ for the bat data set under the body-fixed coordinate system with the constant speed maneuver. . . . . . . . . . . . . . . . . . 133

5.166 The $\Delta V$ distribution for seven influential neighbors and $\delta_{\text {attraction }}=3 \mathrm{~m}$ for the bat data set under the body-fixed coordinate system with the constant speed maneuver. . . . . . . . . . . . . . . . . . . . . . . 13

5.167 The $\Delta V$ distribution for three influential neighbors and $\delta_{\text {attraction }}=$ infinity for the bat data set under the body-fixed coordinate system with the variable speed maneuver. . . . . . . . . . . . . . . . . . . . 135

5.168 The $\Delta V$ distribution for five influential neighbors and $\delta_{\text {attraction }}=$ infinity for the bat data set under the body-fixed coordinate system with the variable speed maneuver. . . . . . . . . . . . . . . . . . . . 136

5.169 The $\Delta V$ distribution for seven influential neighbors and $\delta_{\text {attraction }}=$ infinity for the bat data set under the body-fixed coordinate system with the variable speed maneuver. . . . . . . . . . . . . . . . . . . . . 136

5.170 The $\Delta V$ distribution for the maximum number of influential neighbors and $\delta_{\text {attraction }}=$ infinity for the bat data set under the body-fixed coordinate system with the variable speed maneuver. . . . . . . . . . . . . . . . 137

5.171 The $\Delta V$ distribution for the maximum number of influential neighbors and $\delta_{\text {attraction }}=3 \mathrm{~m}$ for the bat data set under the body-fixed coordinate system with the variable speed maneuver. . . . . . . . . . . . . . . . . 137

5.172 The $\Delta V$ distribution for the maximum number of influential neighbors and $\delta_{\text {attraction }}=1 \mathrm{~m}$ for the bat data set under the body-fixed coordinate system with the variable speed maneuver. . . . . . . . . . . . . . . . . 138

5.173 The $\Delta V$ distribution for the maximum number of influential neighbors and $\delta_{\text {attraction }}=0.5 \mathrm{~m}$ for the bat data set under the body-fixed coordinate system with the variable speed maneuver. . . . . . . . . . . . . . . . . 138

5.174 The $\Delta V$ distribution for seven influential neighbors and $\delta_{\text {attraction }}=3 \mathrm{~m}$ for the bat data set under the body-fixed coordinate system with the variable speed maneuver. . . . . . . . . . . . . . . . . . 139 


\section{LIST OF TABLES}

Table Number

Page

3.1 This table gives the zone ranges for the giant danios in body lengths. The maximum repulsion distance is denoted $\delta_{r}$, and the minimum attraction distance is denoted $\delta_{\text {attraction }}$. End-to-end, fish are both within a $30^{\circ}$ cone in front of, or behind, one another. Side-by-side, neither fish is with a $30^{\circ}$ cone in front of, or behind, one another. . . . . . . . . . . . . . 15

5.1 Table outline the different barn swallow data sets. . . . . . . . . . . . 67

5.2 The average number of frames with at least one conflict seen by an individual bat in the global coordinate frame for a constant speed maneuver. The top parameter is the maximum sensing range in meters, and the left parameter is the number of neighbors being sensed. A total of 1179 frames were used in the analysis. . . . . . . . . . . . . . . . . . . . . 124

5.3 The average time, in seconds, in the global coordinate frame scenario with the constant speed maneuver for an individual bat to shift its velocity vector closest to that of the optimal. The top parameter is the maximum sensing range in meters, and the left parameter is the number of neighbors being sensed. . . . . . . . . . . . . . . . . . . 127

5.4 The average number of frames with at least one conflict seen by an individual bat in the global coordinate frame for a variable speed maneuver. The top parameter is the maximum sensing range in meters, and the left parameter is the number of neighbors being sensed. A total of 1179 frames are used in the analysis. . . . . . . . . . . . . . . . . . . 127

5.5 The average time, in seconds, in the global coordinate frame scenario with the variable speed maneuver for an indivual bat uses to shift its velocity vector closest to that of the optimal. The top parameter is the maximum sensing range in meters, and the left parameter is the number of neighbors being sensed. . . . . . . . . . . . . . . . . . . . 127

5.6 The average number of frames with at least one conflict seen by an individual bat in the body coordinate frame for a constant speed maneuver. The top parameter is the maximum sensing range, and the left parameter is the number of neighbors being sensed. A total 1179 frames are used in the analysis.130 
5.7 The average time, in seconds, in the body coordinate frame scenario with the contstant speed maneuver for an individual bat used to shift its velocity vector closest to that of the optimal. The top parameter is the maximum sensing range in meters, and the left parameter is the number of neighbors being sensed. . . . . . . . . . . . . . . . . . . . 134

5.8 The average number of frames with at least one conflict seen by an individual bat in the body coordinate frame for a variable speed maneuver. The top parameter is the maximum sensing range, and the left parameter is the number of neighbors being sensed. A total 1179 frames are used in the analysis.135

5.9 The average time, in seconds, in the body coordinate frame scenario with the variable speed maneuver for an individual bat used to shift its velocity vector closest to that of the optimal. The top parameter is the maximum sensing range in meters, and the left parameter is the number of neighbors being sensed. . . . . . . . . . . . . . . . . . 139 


\section{ACKNOWLEDGMENTS}

I wish to express sincere appreciation to the Animal Inspired Robust Flight with Outer and Inner Loop Strategies, Multidisciplinary University Research Initiative for funding this research. I would also like to thank the Hedrick Lab for the collection and processing of the barn swallow data as well as the Kunz Bat Lab and Video Computing Group for the collection and processing of the cave bat data. I would like to thank Katherine Midkiff for

her help with running all the data sets through the various algorithms. Finally, I wish to thank Kristi Morgansen and the Nonlinear Dynamics Control Lab for all of their support. 


\section{DEDICATION}

to my parents, Mark and LeAnn

and my grandparent, Larry and Shirley 



\section{Chapter 1}

\section{INTRODUCTION}

The use of autonomous vehicles has been growing for several years with system capabilities rapidly improving with respect to endurance, efficiency, onboard sensing and decision making. However, such systems lack the agility and onboard perception demonstrated by many biological species such as fish, birds and bats. In particular, biological systems appear to have better performance in terms of response time on short time scales when avoiding unexpected obstacles than do engineered systems. In order to aid in the development of bio-inspired deconfliction algorithms to inform studies of biological species and to improve the capabilities of engeered systems, one approach is to study the behavior of biological systems relative to models of motion that can be translated to engineering control system design. In particular, the work in this thesis addresses the study of reactive obstacle avoidance on short time scales in a range of biological species. The end goal of this work is not just to explore the particulars of obstacle avoidance in specific species that can be directly emulated in comparable engineered systems, but to abstract general principles that can be applied in a broad range of engineering contexts that may have little physical similarity to the biological systems from which the principles were derived. Three biological species are considered in this thesis; fish (Danio aequipinnatus), barn swallows (Hirundo rustica), and cave bats (Myotis Velifer).

\subsection{Engineered Deconfliction}

Collision avoidance, or conflict resolution, is an active area of research. A collision occurs when two agents end up inside a predetermined minimum seperation distance from one another. A conflict between two agents is detected if at some future time a collision will occur fs the two agents maintain their current heading. In Kuchar and Yang's survey of papers [19], conflict resolution is divided into three methods: prescribed, optimization, and 
force field. A prescribed method is a set of protocols that an agent must follow [17, 23, 27]. The benefit to a prescribed method is that it can decrease response time; no calculations are involved. Prescribed maneuvers can be less effective than real time calculation methods as they cannot be modified to address specific situations. The optimization methods generally combine a kinematic model and a set of cost metrics to solve for trajectories with the lowest cost $[9,25]$. The costs that can be minimized can be economic costs like fuel and money or they can be workload. The complexity of the functions can make optimization functions difficult to understand, and they can become computationally intensive. The third method, force fields, treats the agents like charged particles $[8,16,26,31]$. Modified electrostatic equations are used to generate maneuvers. The force field approach uses fairly simple equations, but discontinuities can develop in the resolution maneuvers.

This thesis uses collision cones, also known as velocity obstacles, as the engineering collision avoidance method for which the biological species collision avoidance is compared. Collision cones are defined as a set of velocities that will cause a collision with another object [5]. In particular, the algorithm seen here is based on the Distributed Reactive Collision Avoidance (DRCA) by Emmitt Lalish [20] and [21]. The portion of the DRCA that this thesis uses is optimization based.

\subsection{Biological Systems}

By comparing multiple species to an engineered model, we gain insight into which animal characteristics are most closely aligned with the model. Also, different species have different outer loop goals to accomplish that are more closely related to the different scenarios that the engineering models were created for, i.e. foraging could be analgous to a searching algorithm. Behavior models for schooling fish have been validated in $[4,15,28,30]$. Network models have been found used to describe information transfer in golden shiners [7]. Groups of locust have been have been compared to a self-propelled particle model [2]. Ant behavior models have been explored in [6, 32].

The three species compared in this thesis, giant Danios, barn swallows, and cave bats, are studied because of their maneuverablity and social interactions. Two main behavioral criteria are explored in this thesis: the number of influential neighbors [1, 29] and a maximum 
sensing range which is based on the zonal model [3, 28, 29, 30].

\subsection{Contributions}

The work in this thesis is one of the few direct analyses of engineering based decision algorithms relative to biological species. In addition, this analysis is performed across multiple species. This comparison indicates the following characteristics: (1) a strong correlation exists between the deconfliction algorithm being considered here, (2) different species demonstrate weaker or stronger tendencies toward the use of variable speed to avoid collisions, and (3) different species demonstrate collision avoidance relative to different data reference frames.

\subsection{Outline of Thesis}

The remainder of the thesis is organized as follows. The biological species analyzed in this work are discussed in Chapter two. In Chapter three, the perception and reaction guidelines to be applied to the biological species are presented. The collision avoidance algorithm considered here is presented in Chapter four along with two coordinate system options for application of the method. Results of algorithm evaluation and discussion are presented in Chapter five, and conclusions and future work are given in Chapter six. 
Chapter 2

\section{BIOLOGICAL SPECIES}

This chapter presents the three biological species that are analyzed in this thesis, giant danios (Danio aequipinnatus), barn swallows (Hirundo rustica) and cave bats (Myotis velifer. Three-dimensional trajectory data were collected from each of the species.

\subsection{Giant Danios - Schooling Fish}

Giant danios are socially gregarious freshwater semitropical fish that are between four and six $\mathrm{cm}$ in length and have a mass of $1.5-3 \mathrm{~g}$. Socially gregarious fish tend to move together and stay in groups. Giant danios have a maximum velocity of approximately $12 B \mathrm{Ls}^{-1}$ [30]. These fish demonstrate agile maneuverablity as well as quick responses on short time and distance scales.

The tank in which the giant danios were filmed was two by three meters with a one meter depth. The data were collected in a lab environment using four video cameras placed above the tank filming at 30 frames per second. Recordings were made of groups of 15 fish in which some of the fish were trained to respond to a food cue. When this cue was initiated, the trained fish would begin searching for food. Five sets of trajectory data were collected, each set having three segments. The five sets correspond to the number of trained and untrained fish in the school: 15-0, 12-3, 9-6, 6-9, 0-15. The three segments are each one minute long and correspond to activity before the cue (Beg), during the cue (Mid), and after the cue (End). These data were collected at the University of Washington in the Birdfish Lab headed by Julia Parrish. The video data were then processed into three dimensional trajectories using the software package tracker3D [11]. Figure 2.1 depics a typical data set of the 15 trajecties where each color represents a different fish trajectory. The velocity data, calculated using a forward difference method, from a typical schooling fish is shown in Figure 2.2. More information about the giant danio data collection can be found in [22]. 


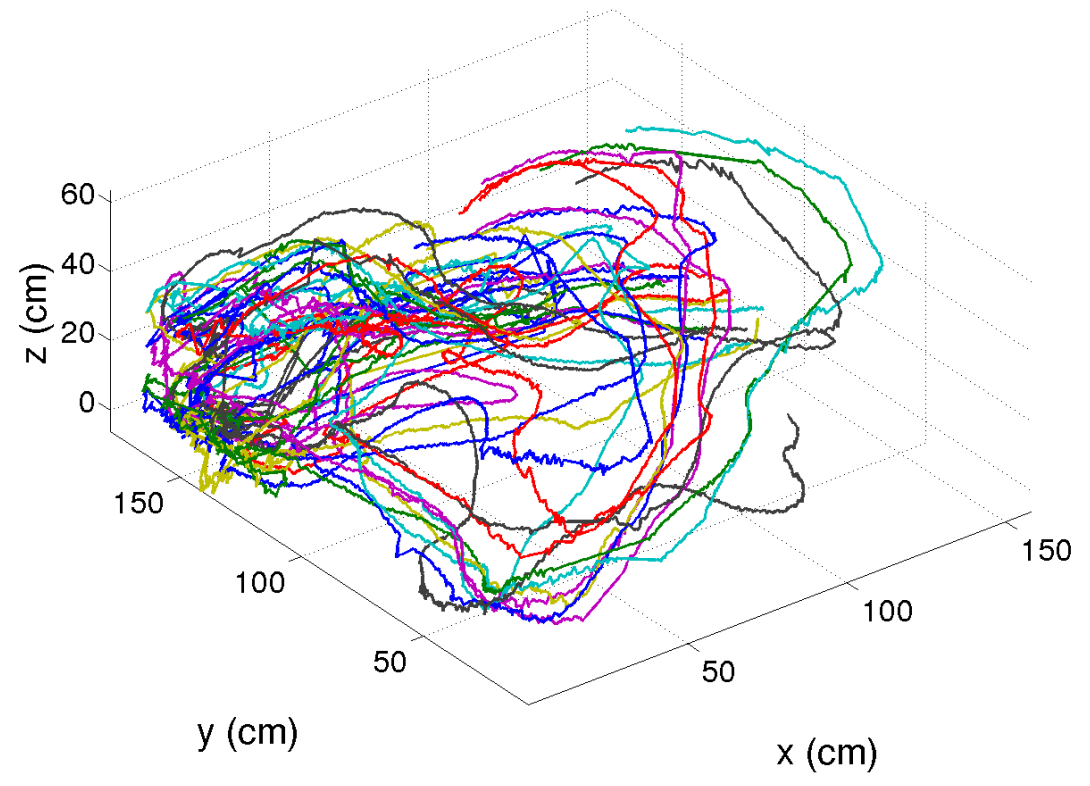

Figure 2.1: This figure shows the three dimensional position data for the giant danios during the beginning segment of the 6-9 data set. Each colored line represents a trajectory of a different fish. The $x-y$ plane forms the horizontal while the $z$-direction points antiparallel to gravity. This data segment contains trajectories for 15 giant danios.

\subsection{Barn Swallows - Maneuvering Birds}

Barn swallows have a body length of approximately 0.18 meters and a wing span of 0.32 meters. The swallows were filmed in the wild at a farm in Yanceyville, North Carolina using three IDT N5 cameras (Integrated Design Tools Inc.) running at $100 \mathrm{~Hz}$ with a frame resolution of 2336 x 1728 pixels. Nikon $20 \mathrm{~mm} \mathrm{f} / 28 \mathrm{AF}$ lenses were used on the three cameras. A 1.0 meter length reference object was used for calibrating the cameras. The three dimensional trajectories were created from custom tracking routines in MATLAB via triangulation from the three cameras [14]. The position errors were estimated from the triangulation residual and used with a smoothing spline to produce minimum-acceleration trajectories for each swallow trajectory. Barn swallow data was collected by the Hedrick Lab at the University of North Carolina at Chapel Hill. The data were collected in July-August 

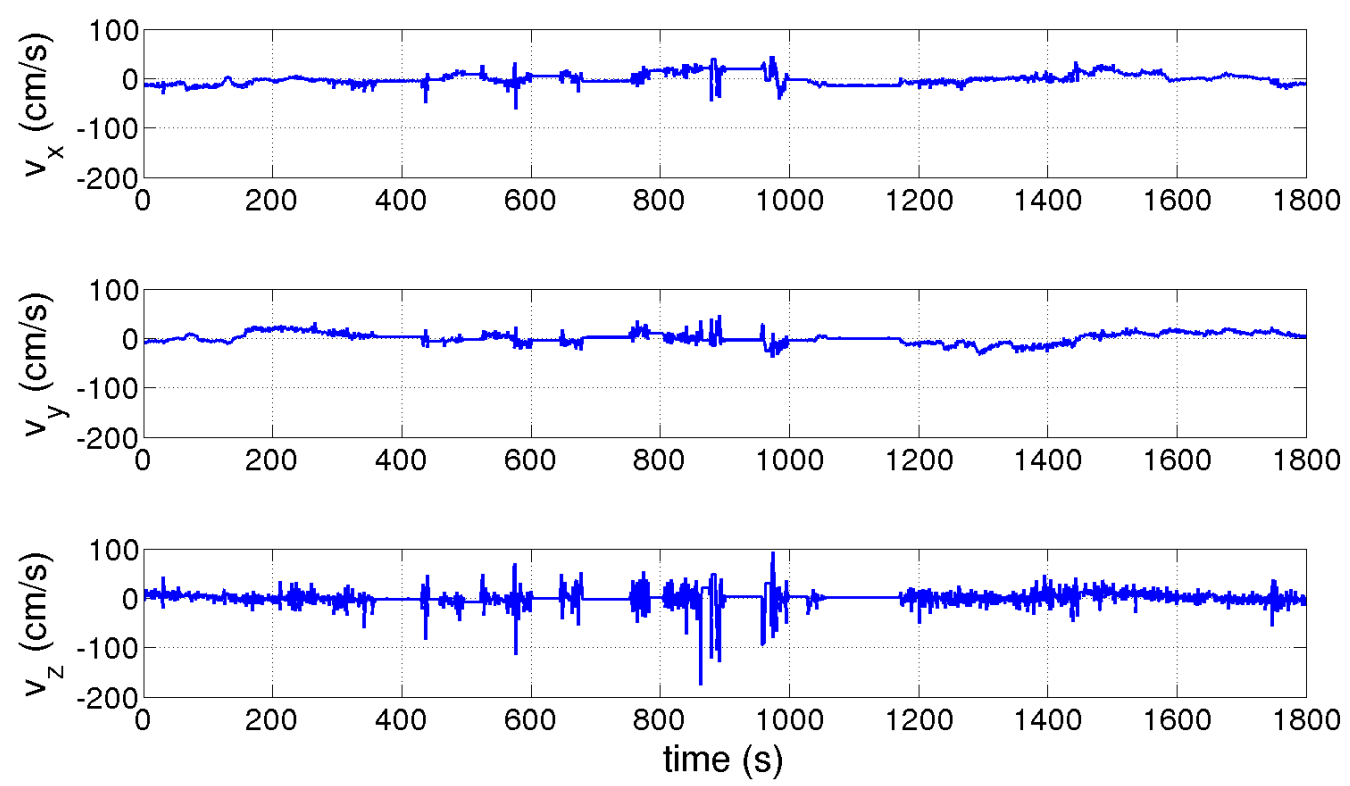

Figure 2.2: The $x-, y-$, and $z$-velocity data for a typical giant danio during the beginnning segment of the 6-9 data set. The topmost plot is the $x$-velocity, the center plot is the $y$-velocity, and the bottom plot is the $z$-velocity. The data segment is one minute long at 30 frames per second.

2011 and April 2012.

Barn swallows are not socially gregarious and are usually found in small groups. Barn swallows do, however, interact with each other, such as through pursuit-evasion. These birds demonstrate fast time scales. When interacting with static obstacles, the barn swallows have short reaction time and distance scales. Data collected for the swallows included both larger and smaller numbers of animals with and without static obstacles. A single flock of 23 birds was recorded for 4.75 minutes at 100 frames per second, and five other data sets were recorded with the number of swallows ranging from two to twelve. Figure 2.3 is a plot of the trajectories of the 23 bird flock, and Figure 2.4 is the velocity data of a single bird from that flock. The velocity data was determined using a finite difference method. The static obstacles included grain silos and roofs. The silos have a geometry that fits inside a cylinder with a six foot height and a radius of 1.35 meters. The roof lines were 38.7 meters and 39.7 
meters in length and were 2.4 meters off the ground. Additionally, one set of data had a truck driving through the field of view. The front right wheel and the front right roof of the truck were recorded in the data.

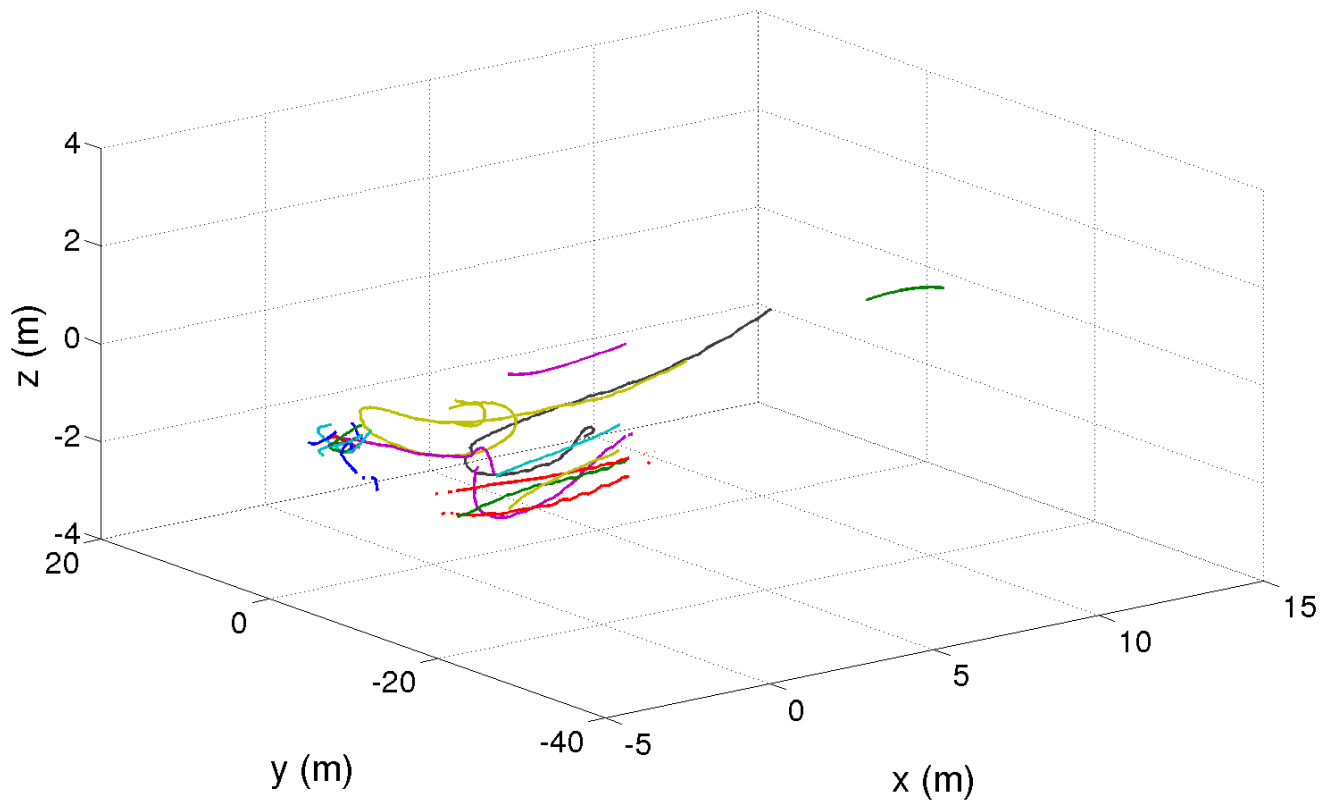

Figure 2.3: One of the data sets for position data of barn swallows. The trajectories of 23 barn swallows are shown. Each colored line represents the trajectory of a different bird. The $x-y$ plane is the horizontal plane, while the $z$-direction is antiparallel to gravity.

\subsection{Cave Bats - Hunting Bats}

The data analyzed here include one set of cave bat trajectory data. The bats are approximately 0.09 meters long, have a wing span of 0.084 meters, a mass of 12 grams, and travel at a maximum velocity around $7 \mathrm{~ms}^{-1}$. The cave bats were filmed in their natural habitat. These bats can be found in large groups when emerging from a cave or in small groups when foraging. When traveling in a group, the cave bats move in a coherant structure, and the bats do not appear to run into one another. The main sensing modality of this species of bat is echolocation. Three mid-infrared FLIR SC8000 cameras filming at 131.5 frames per second and with a resolution of 1024x1024 pixels captured the bat flights in 

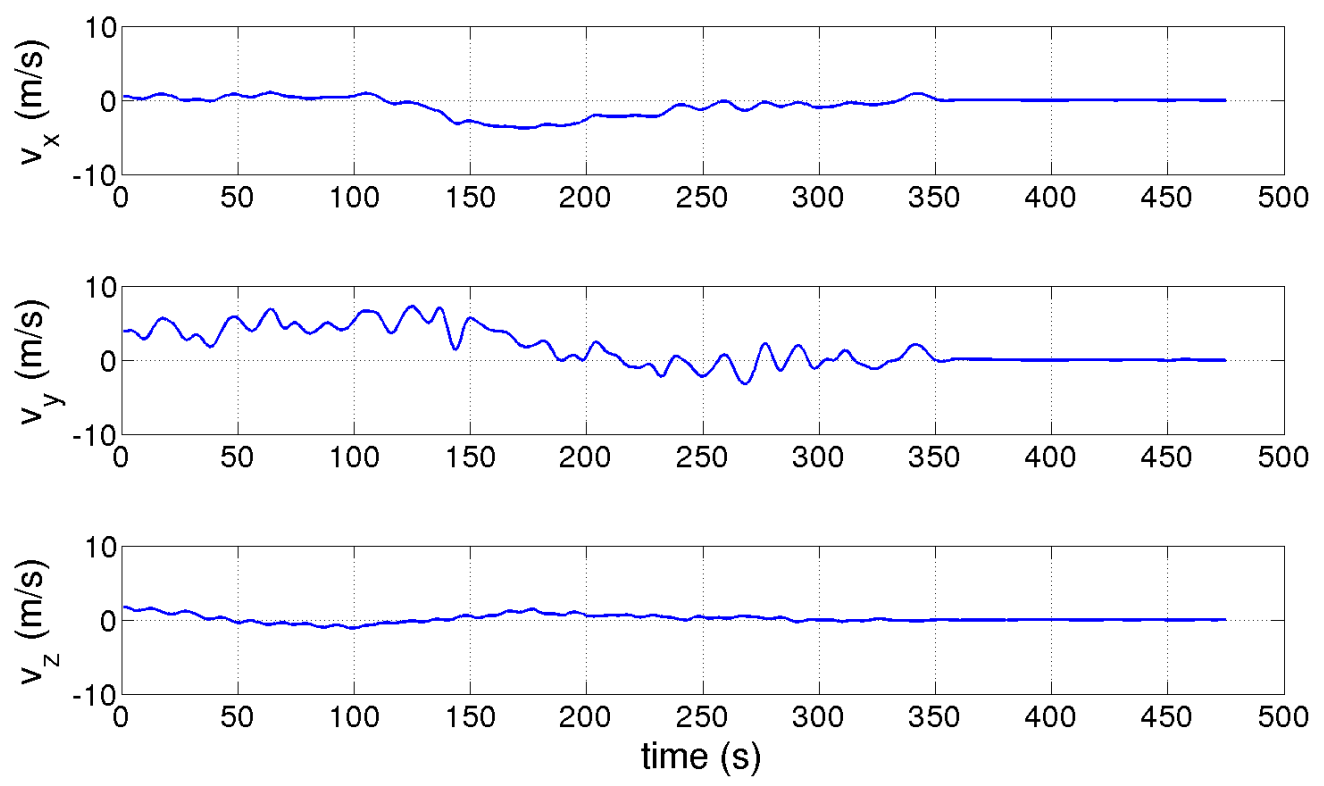

Figure 2.4: This figure plots the different comonents of the velocity data for a typical barn swallow during the flock of 23 birds data set. The topmost plot is the $x$-velocity, the center plot is the $y$-velocity, and the bottom plot is the $z$-velocity. The Flock data set is about 4.5 seconds long at 100 frames per second.

their natural habitat. A calibration wand was waved around in the overlapping fields of view of the cameras to generate calibration point pairs. The across-view and across-time correspondences were established by hand using an in-house semi-automated tool. Once the initial correspondences were established, the tool used a rudimentary tracker to follow the objects through the scene; the annotators could correct the tracker at any point. From the manually annotated correspondences, the three-dimensional points were recontructed using the standard linear algebra based on least squares [12].

Only one bat data set was analyzed in this thesis. The data set contains a total of 20 bat trajectories, with up to 10 bats in the field of view at any one time. The trajectory data for the data set is in Figure 2.5, and the velocity of a typical single bat is in Figure 2.6. Within the field of view were two static obstacles the bats needed to avoided, a pole of length 1.6 meters and a vine of length 0.2 meters. The bats can fly over the pole but not 
the vine. The bat data were collected by the Kunz Bat Lab and processed by the Image and Video Computing Group at Boston University.

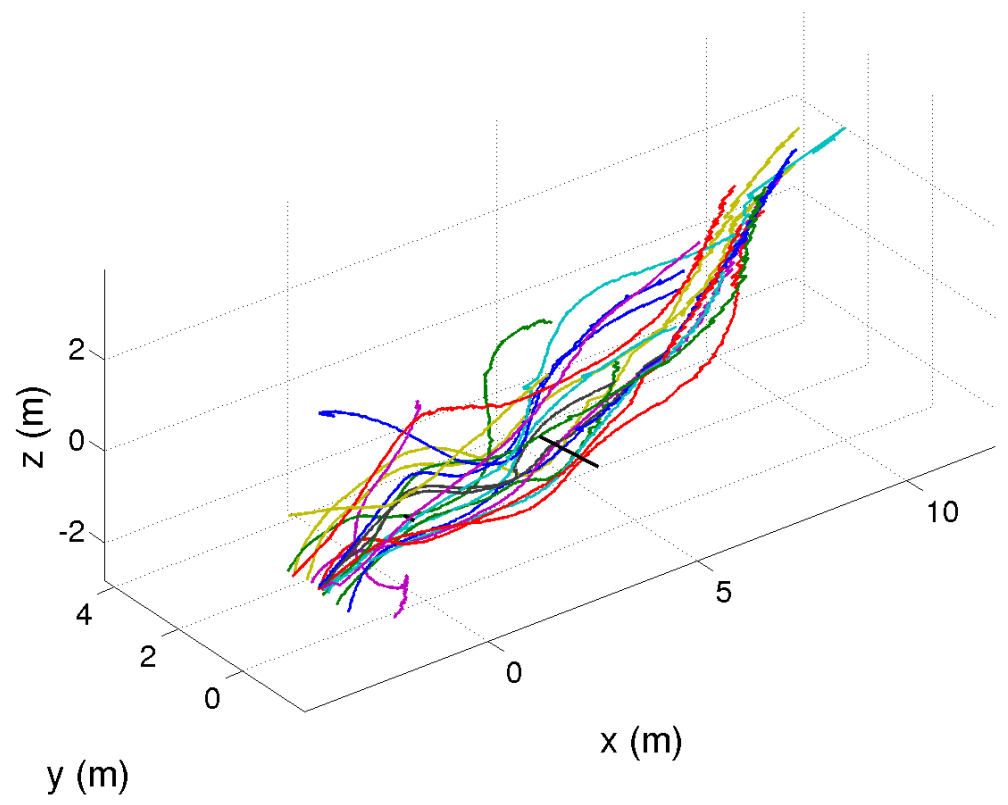

Figure 2.5: This figure shows the three dimensional position data for bats during the data set. A total of 20 bat trajectories form this data set. Each colored line represents a different bat's trajectory. The black lines represent the static obstacles, a vine and a pole. The $x-y$ plane forms the horizontal plane while the $z$-direction is antiparallel to gravity. 

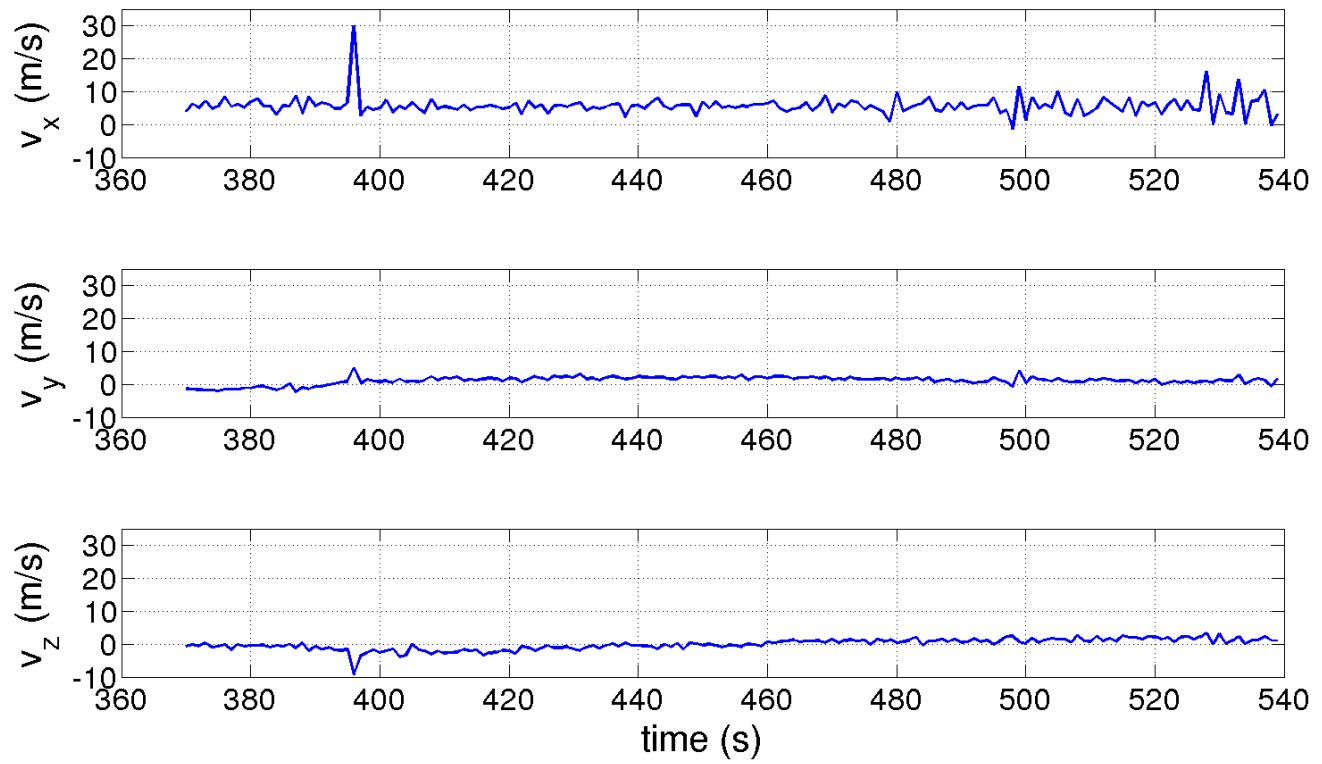

Figure 2.6: This figure shows plots of the different components of the velocity data for a typical cave bat during the data set. The topmost plot is the $x$-velocity, the center plot is the $y$-velocity, and the bottom plot is the $z$-velocity. This data set is about four seconds long at 131.5 frames per second. 


\section{Chapter 3}

\section{PERCEPTION AND REACTION}

Engineered systems and biological systems both need sensors to provide information on what and where obstacles are in the environment around them. In particular for collision avoidance, the sensors need to tell the system how far away, how fast, and in what direction the obstacles are traveling. Visual, auditory and mechano sensors are used in

biological systems. Some of the sensors engineered systems use include cameras, radars, and accelerometers.

One hypothesis that biologist are using to describe how animals stay together without colliding with one another while traveling in a group is that of the zonal model. The zonal model is a set of regions in wach of which animals follow a particular set of behavioral rules with respect to neighbors in that region. Another theory about animal interation behavior revolves around a specific number of influential nieghbors. In this thesis, the zonal model, number of influential neighbors and a hybrid of the two theories is used in the analysis.

\subsection{Zonal Model}

A popular framework in which interactions between animals have been considered in the past several years is that of zonal models of behavior [30]. Zonal models are composed of three regions defined by the radial distance from the animal's center of mass: attraction, alignment and repulsion [3]. A dipiction of the zonal model with all three regions and blind spot is shown in Figure 3.1. The particular aspect of animal interaction being studied here, collision avoidance, lies in the regime of the reactive repulsion and alignment component of zonal models. Any obstacle in the attraction zone is ignored as the animal would want to move toward the object and thus override any collision avoidance behavior.

The inner most zone is the repulsion zone located within a radius of $\delta_{r}$ from the animal's center of mass. If an animal has an object in the repulsion zone, the animal will move away 
from that object. The next zone is the alignment zone. The alignment zone, between $\delta_{r}$ and $\delta_{\text {alignment }}$, contains objects with which the system wishes to align its heading so that the objects become directionally aligned in their movement. The furthest zone is the attraction zone, located between $\delta_{\text {alignment }}$ and $\delta_{\text {attraction. }}$. Any objects in the attraction zone will cause the animal to move toward those objects. Any objects outside of the attraction zone are either not sensed or are ignored.

In particular for giant danios, the repulsive and attraction zone parameters can be found in Figure 3.1. An end-to-end configuration of animals refers to the animals being mutually within a 30 degree cone to the front of, or behind, one another. A side-to-side configuration means that neither of the animals are within a 30 degree cone to the front of, or behind, the other [29].

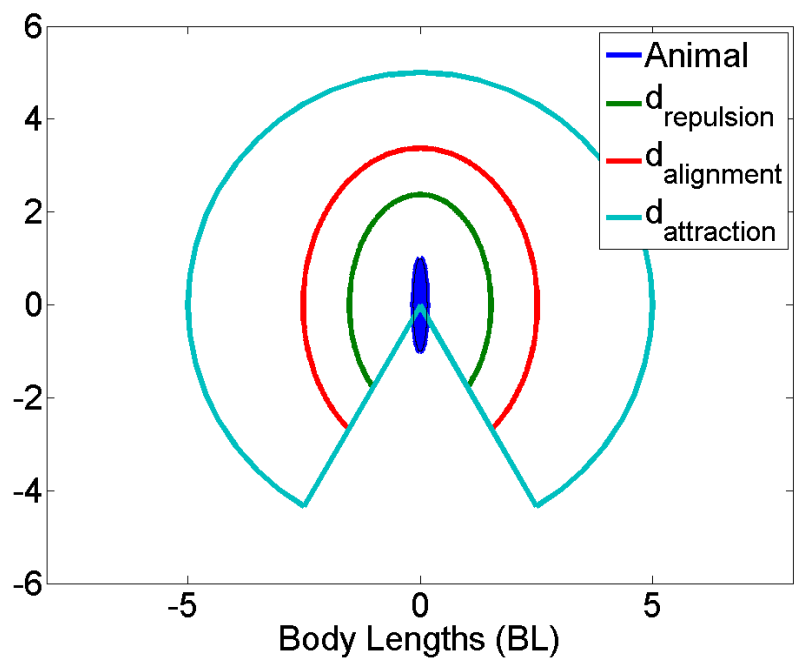

Figure 3.1: This figure is a depiction of an example of the biological zonal model. The central blue ellipsoid is the biological animal. The green line is the outer boundary of the repulsion zone. The alignment zone's outer boundery is the red line. The outer boundary of the attraction zone is represented by the cyan colored line. The gap in the oval at the bottom of the biological represents the sensing blind spot. Everything outside the attraction zone is ignored.

These zones can be mapped to engineered systems that are working in a network. For two vehicles trying to avoid collisions, a repulsion zone can be constructed. For scenarios 
where vehicles need to be within a certain distance of one another such as for sensing or communication purposes, an alignment zone can be constructed based on the optimal relative distances between the vehicles. When a vehicle senses that it is moving too far away from another, an attraction zone can be effected to cause the vehicle to adjust its heading to move back toward its fellow vehicles in the network.

\subsubsection{Maximum Range}

In this approach to obstacle detection, a limit is set on the maximum distance range over which obstacles will be considered. This distance is referred to as the metric range. For the purposes of this thesis, the maximum range means that any object within some radius is considered an obstacle that needs to be avoided. The easiest geometry to use is a circle of constant radius. Other possible geometries include ellipsoids or geometries with missing areas that represent blind spots or other regions without sensing. In this thesis, a constant

radius, $\delta_{\text {attraction }}$, was used for the range of influence. For each species, the maximum radius used in the algorithm was varied to numerically determine the sensing range of the three species.

\subsection{Number of Influential Nearest Neighbors}

The number of nearest neighbors can be thought of as limited processing power for an engineered system. In this characteristic, a fixed number of neighbors are labeled as influential. The animal does not pay attention to all objects around it, just the nearest ones. The number of influential nearest neighbors is referred to as the topological range.

Schooling fish have been shown to be influenced by some number of nearest neighbors [29]. The number of neighbors is based not only on the number of fish in the school but also the number of fish in the group. The school is the total number of fish, and a group is defined as individuals within five body lengths of each other. Using data regression [29], the

nominal group size, $\mathrm{G}$, for a school or size $\rho$ is $G=47.446\left(1-e^{-0.026 \rho}\right)$, and the number of influential neighbors can then be determined from $G=3.10 \lambda-6.69$. Here, the school size was $\rho=15$ giving a group size $G=15$ and a number of influential neighbors of $\lambda=7$. 
In flocking birds, the topological range is more important than the metric range for interactions between neighbors. The average number of nearest neighbors that influence a starling is $6.5 \pm 0.9 \mathrm{SE}$, where $\mathrm{SE}$ is the standard error [1]. This result was determined from flocks ranging from hundreds to thousands of starlings as part of the STARFLAG project.

For a colony of bats flying together, the literature is lacking in information about the number of influential neighbors.

\subsection{Hybrid Criteria: Merging of Maximum Range and Nearest Neighbors}

In this combination of the zonal model and the nearest neighbors approach, only the influential neighbors within the maximum range are analyzed. If an influential neighbor is outside the maximum range, then that neighbor is ignored.

The hybrid approach is the most realistic approach for both biological animals and engineered systems. A maximum distance is set over which sensors can provide usable data. This maximum distance can also be used to limit the amount of data recorded. Too much

information can slow down the calculations and estimations an on-board computer would need to perform. 


\begin{tabular}{l|cr}
\hline Relative orientation & $\delta_{r}$ & $\delta_{\text {attraction }}$ \\
\hline End-to-end & 2.37 & 3.37 \\
Side-by-side & 1.52 & 2.52 \\
\hline
\end{tabular}

Table 3.1: This table gives the zone ranges for the giant danios in body lengths. The maximum repulsion distance is denoted $\delta_{r}$, and the minimum attraction distance is denoted $\delta_{\text {attraction. }}$ End-to-end, fish are both within a $30^{\circ}$ cone in front of, or behind, one another. Side-by-side, neither fish is with a $30^{\circ}$ cone in front of, or behind, one another. 


\section{Chapter 4}

\section{COLLISION AVOIDANCE}

The analytical approach used here is based on a deconfliction algorithm that uses collision cones (also termed velocity obstacles) to determine likelihood of collision. Collision cones are a set of velocities that cause a conflict between two entities. The Distributed Reactive Collision Avoidance (DRCA) algorithm [20], detailed below, provides a behavioral response to detection of likely collision that is optimal in the sense of minimal change in velocity [20]. To compare this algorithm to biological response, we take the following approach using mathematical models of deconfliction which have provable behavior (desirable characteristics for engineered systems) to probe biological obstacle avoidance characteristics. The amount of correlation between the analytical models and biological behavior will be explored in terms of properties such as change in speed, change in flight direction, time of response and different objects being avoided (same species, different species, fixed objects, ect). The details of the collion cone based DRCA algorithm are presented below including how conflict is determined and maneuver specification for avoiding colliion based on constant or variable velocity magnitude changes. Also discussed are implementation variations based on the reference frame in which the conflict determination and avoidance are calculated.

Collision cones are calculated between each biological animal and each obstacle in the field of view. If a conflict exists between the animall and at least one obstacle, this algorithm calculates the optimal velocity vector that would move the animal out of conflict using the smallest change in velocity. The smallest change in velocity is calculated by taking the norm of the difference between the original velocity vector and the velocity vector that would move the animal out of conflict. The calculated optimal velocity vector is then compared to how the velocity vector of the biological animal actually changes. 


\subsection{Coordinate Systems}

The three-dimensional global coordinate system used in this thesis has the $x-$ and $y-$ axes forming the horizontal plane and the $z$-axis pointing in the vertical direction opposite gravity. The collision avoidance algorithm to which the biological systems were compared is a planar algorithm, and all of the guarantees the algorithm provides currently only hold for planar data. In order to use the algorithm, three-dimensional data for biological flight was projected into one of two different planes to make it two-dimensional. The first projection was a simple orthogonal projection into the horizontal plane perpendicular to gravity, which will be called the global coordinate system. The second projection first transformed the three-dimentional data from inertial coordinates to body-fixed coordinates, $x_{b}, y_{b}, z_{b}$, with $x_{b}$ pointing in the direction of motion of the entity, $y_{b}$ dirctly to the left and perpendicular to $x_{b}$, and $z_{b}$ pointing up to form a right-handed system. An orthogonal projection was then applied to restrict the data to the $x_{b}-y_{b}$ plane.

\subsubsection{Two Dimensional Projection: Global Coordinates}

In the first approach, all of the trajectories were projected into the standard Euler horizontal $x-y$ global plane. This projection was done by removing the $z$-component of the position and velocity data, as shown in Figure 4.1. This approach is most likely appropriate when the the biological systems have a small deviation in the $z$-direction of their vectors during flight. While this projection is simple, and requires less processing power than the method discussed below, the information about the vertical relative distance is lost.

\subsubsection{Two Dimensional Projection: Body Coordinates}

To take into consideration the effect of variations in $z$ component of the position and velocity, a second coordinate system and projection were used and compared to the first. This second coordinate system was a body fixed frame, as described above. First the system was transformed to body coordinates, and then it was projected down into the $x_{b}-y_{b}$ body plane, as shown in Figure 4.2 .

From the velocity vector in global coordiantes, the yaw and pitch of the body-fixed 


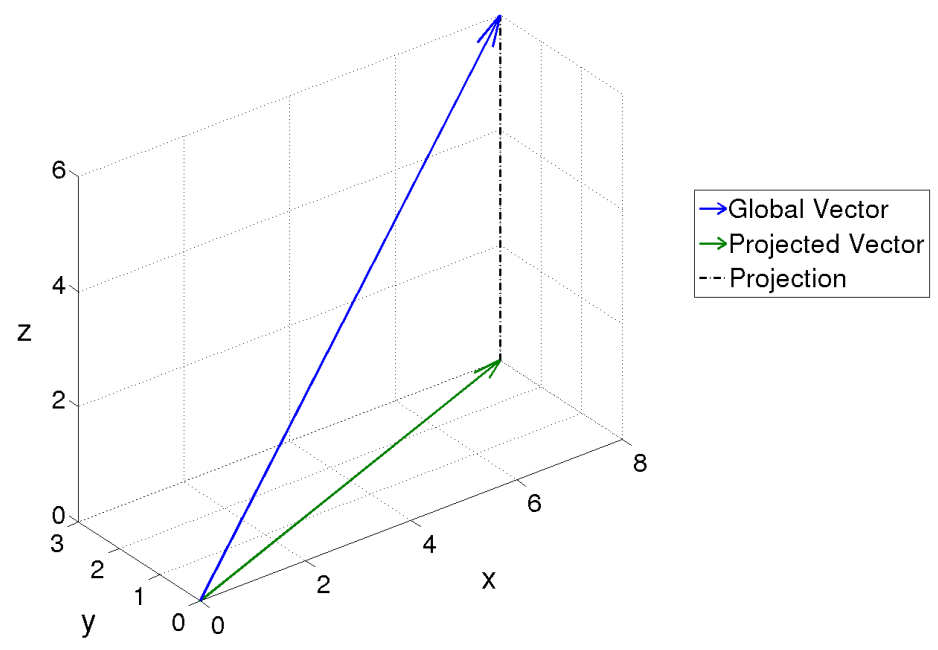

Figure 4.1: This figure is an example of a global projection. The blue arrow is the original vector, and the green is the vector projected into the horizontal $x-y$ plane.

coordinate system can be determined. Yaw is defined as the heading angle of the biological system in the $x-y$ global plane, $y a w=\arctan \left(\frac{\mathbf{v}_{i, y}}{\mathbf{v}_{i, x}}\right)$. Pitch is the angle created between the velocity vector and the $x-y$ global plane, pitch $=-\arctan \left(\frac{\mathbf{v}_{i, z}}{\sqrt{\mathbf{v}_{i, x}^{2}+\mathbf{v}_{i, y}^{2}}}\right)$. Roll was set to zero. This assumption was made because no information was captured about the roll angle of the animal's head. Ideally, the orientation of each animal's head would be used to deteremine the body coordinate frame, but at this time that level of detail is not available.

Given a velocity vector, $v$, in global coordinates, the cooresponding vector represented in body coordinates, $v_{b}$, was constructed using the relation $v_{b}=R v$ where $R=R_{x} R_{y} R_{z}$, $R \in S O(3) \subset \mathbb{R}^{3 \times 3}$, and

$$
\begin{gathered}
R_{x}=\left[\begin{array}{ccc}
1 & 0 & 0 \\
0 & \cos (\text { roll }) & \sin (\text { roll }) \\
0 & -\sin (\text { roll }) & \cos (\text { roll })
\end{array}\right] \\
R_{y}=\left[\begin{array}{ccc}
\cos (\text { pitch }) & 0 & -\sin (\text { pitch }) \\
0 & 1 & 0 \\
\sin (\text { pitch }) & 0 & \cos (\text { pitch })
\end{array}\right]
\end{gathered}
$$




$$
R_{x}=\left[\begin{array}{ccc}
\cos (\text { yaw }) & \sin (\text { yaw }) & 0 \\
-\sin (y a w) & \cos (y a w) & 0 \\
0 & 0 & 1
\end{array}\right]
$$

giving

$$
R=\left[\begin{array}{ccc}
\cos (\text { yaw }) \cos (\text { pitch }) & \sin (\text { yaw }) \cos (\text { pitch }) & -\sin (\text { pitch }) \\
-\sin (\text { yaw }) & \cos (\text { yaw }) & 0 \\
\cos (\text { yaw }) \sin (\text { pitch }) & \sin (\text { yaw }) \sin (\text { pitch }) & \cos (\text { pitch })
\end{array}\right] .
$$

The new vectors must be translated by the position of the biological system to provide relative position and velocity information for the DRCA algorithm:

$$
\begin{gathered}
\mathbf{r}_{i j}=\mathbf{r}_{j}-\mathbf{r}_{i} \\
\mathbf{v}_{i j}=\mathbf{v}_{i}-\mathbf{v}_{j} .
\end{gathered}
$$

Once transformed to the body-fixed coordinate system, the vectors can all be projected into the $x_{b}-y_{b}$ body plane by removing the $z_{b}$ component of the vector. This approach takes into consideration some of the relative vertical distance, but not all of the information is retained. The only way to completely utilize all the relative distance information is to use a three-dimensional algorithm. A drawback to this body-fixed approach is the additional processing power required compared to the first approach.

\subsection{Checking Conflict}

A conflict occurs when the velocity vectors of two agents will cause a collision between those two entities if their behavior does not change. A collision is defined as two entities coming within a minimum separation distance, $d_{s e p, 0}$, of one another. Each entity is modeled as a point mass with volume approximated by a sphere. For two entities, denoted $i$ and $j$, with spherical volumes radius $_{i}$ and radius $_{j}$, the minimum allowable separation distance before a collision occurs is then $d_{s e p}=$ radius $_{i}+$ radius $_{j}+d_{s e p, 0}$.

To analytically determine if a conflict will occur using the DRCA algorithm, the relative position and velocity vectors, $\mathbf{r}_{i j}$ and $\mathbf{v}_{i j}$, are calculated. Next, the calculation of the 


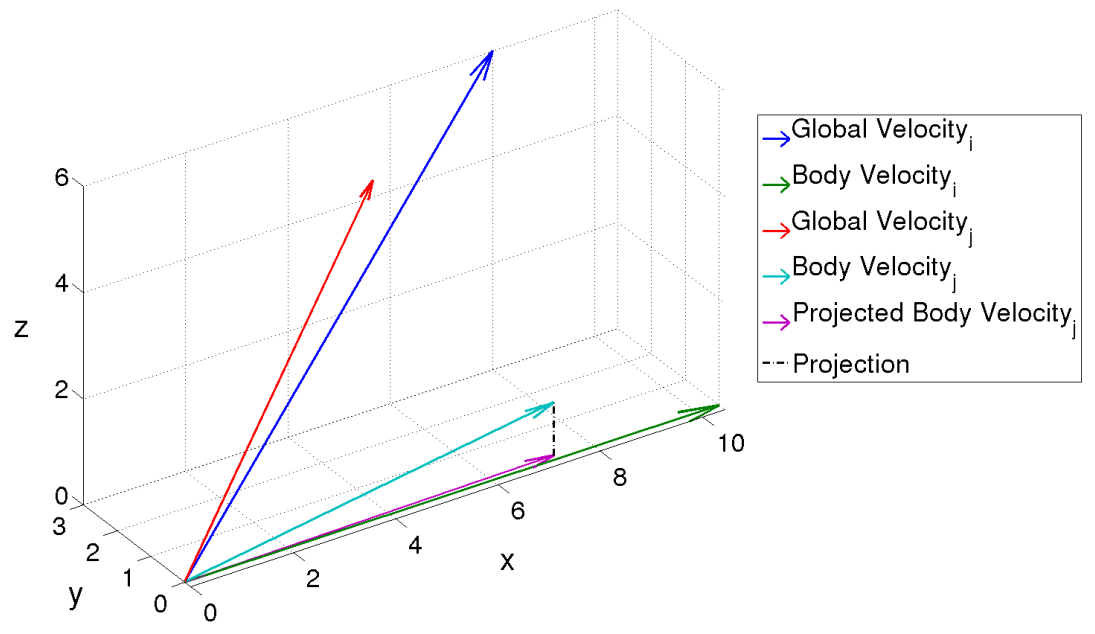

Figure 4.2: This figure is an example of a body coordinate projection. The blue arrow represents the original velocity vector and the green vector is the body coordinate velocity vector. The red arrow is the original velocity vector of the obstacle. The cyan colored vector is the body coordinate obstacle velocity vector, and the magenta is that vector projected into the $x_{b}-y_{b}$ plane.

collision cone parameters $\alpha$ (half the angle of the collision cones) and $\beta$ (the angle between the relative distance and relative velocity vectors), is made:

$$
\begin{gathered}
\alpha=\arcsin \left(\frac{d_{s e p}}{\left\|\mathbf{r}_{i j}\right\|}\right) \\
\beta=\arccos \left(\frac{\mathbf{r}_{i j} \cdot \mathbf{v}_{i j}}{\left\|\mathbf{r}_{i j}\right\|\left\|\mathbf{v}_{i j}\right\|}\right) .
\end{gathered}
$$

If a conflict is predicted, $\beta<\alpha$, then the optimal velocity vector, $\mathbf{v}_{i}^{\prime}$, to bring the entity out of conflict is calculated.

\subsection{Moving Out of Conflict}

Two different maneuvers are considered here to calculate the optimal velocity vector. The first to be discussed is a constant speed maneuver, and the second is a variable speed maneuver. The constant speed maneuver constructs velocity vectors that have the same magnitude as the current velocity vector. This approach is equivalent to only changing the 
heading angle; the vehicle does not slow down or speed up. The variable speed maneuver allows for change in both speed and direction of the velocity and accounts for acceleration or deceleration of an animal or vehicle.

\subsubsection{Constant Speed Maneuver}

The set of all possible solutions of the constant speed maneuver lie on a circle whose radius is equal to the magnitude of the vehicle's velocity (see Figure 4.3). The solutions of interest lie at the intersections between this circle and the edges of the collision cones. A solution is not valid if the intersection between the edge of the cone and the circle is within another collision cone. The solution that is of importance is the one that has the smallest change in velocity. This solution is the optimal choice for this maneuver.

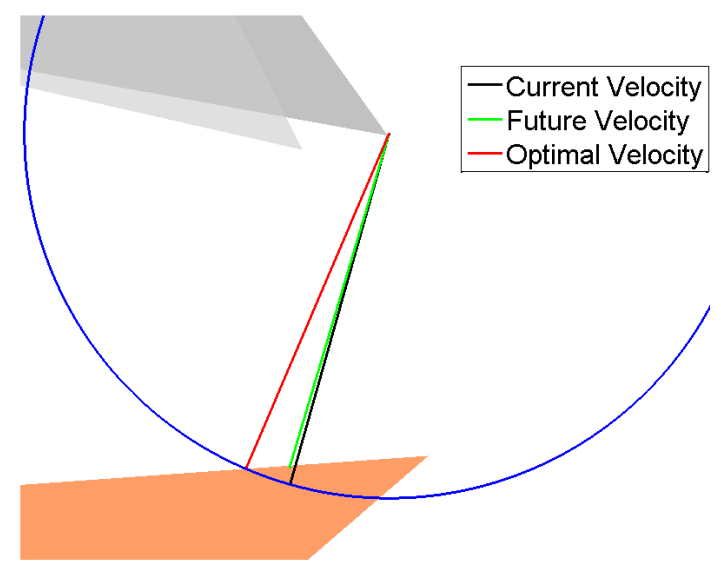

Figure 4.3: This figure is typical graphical representation of the constant speed maneuver. The black vector is the current velocity, the green vectory is the next velocity vector, and the red vector is the optimal solution.

\subsubsection{Variable Speed Maneuver}

For the variable speed maneuver, solutions can be anywhere in the plane so long as a maximum velocity constraint is not broken. The optimal solution is one of the following: 
the nearest point on a single collision cone, the vertex between collision cones, or the vertex between a collision cone and the edge of the allowable space. Note that the space in consideration is velocity space, so these points being considered are velocities.

The nearest point on a single cone is calculated using (4.6) below, where $\hat{\mathbf{c}}$ is the edge of the collision cone and $R$ is a $2 \times 2$ rotation matrix, $R \in S O(2) \subset \mathbb{R}^{2 \times 2}$ :

$$
\begin{aligned}
\hat{\mathbf{c}} & =R( \pm \alpha) \frac{\tilde{\mathbf{r}}}{\|\tilde{\mathbf{r}}\|} \\
\mathbf{v}_{i}^{\prime} & =\hat{\mathbf{c}} \hat{\mathbf{c}}^{\top} \tilde{\mathbf{v}}+\mathbf{v}_{j} .
\end{aligned}
$$

The solution is only valid if the magnitude of the solution is less than the magnitude of the maximum velocity achievable by the system under consideration, $\left\|\mathbf{v}_{i}^{\prime}\right\| \leq v_{i, \max }$. If this solution is not valid, then the next solution is determined by the vertex between the collision cone and the edge of the allowable space, (4.7):

$$
\mathbf{v}_{i}^{\prime}=\mathbf{v}_{j}-\hat{\mathbf{c}} \hat{\mathbf{c}}^{\top} \mathbf{v}_{j} \pm \hat{\mathbf{c}} \sqrt{\left(\hat{\mathbf{c}}^{\top} \mathbf{v}_{j}\right)^{2}-\mathbf{v}_{j}^{\top} \mathbf{v}_{j}+v_{i, \max }^{2}}
$$

The last possible solution to be considered is the vertex between two collision cones, which is found by solving a system of two linear equations. A graphical representation of the variable speed maneuver, Figure 4.4, has the nearest point on a single cone solution. 


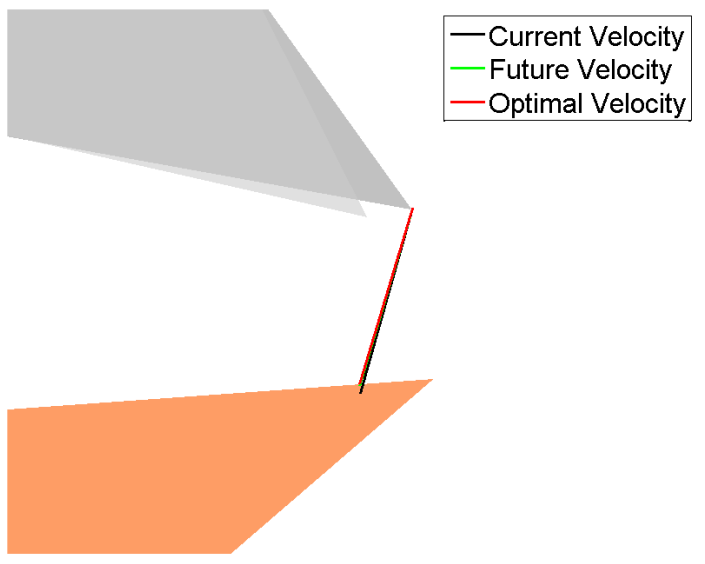

Figure 4.4: This figure is typical graphical representation of the variable speed maneuver. The black vector is the current velocity, the green vectory is the next velocity vector, and the red vector is the optimal solution. 
Chapter 5

\section{ANALYSIS AND RESULTS}

This chapter explores the correlation between biological deconfliction and the analaytical deconfliction method DRCA. The effects on the correlation of characteristics such as coordinate system representation, maneuver types, and sensing range were considered. For each type of animal, giant danios, barn swallows, and bats, the results are shown for both global and body coordinate frame planar projections. For each coordinate frame, the constant and variable speed maneuver were analyzed. Two parameters, number of influential nearest neighbors and the maximum sensing range distance, $\delta_{\text {attraction }}$, were varied to explore their effects. The average time necessary for a biological species to bring itself out of conflict, referred to here as the reaction time, was calculated numerically and compared to values from the literature.

\subsection{Metrics and Correlations}

The main metric used in determining the correlation between biological response and collision cone methods was the norm of the difference in velocity vectors, $\Delta V$, between the predicted optimal velocity vector, as defined by the algorithm, and the actual velocity vector of the biological system:

$$
\Delta V=\left\|V_{i, a c t u a l}^{\prime}-V_{i}^{\prime}\right\|
$$

This metric was applied to each conflict seen by the animal. For each biological species, a $\Delta V$ distribution was created and normalized against the total number of conflicts. These distributions were then averaged to produce a single averaged $\Delta V$ distribution for each data set with particular parameters. The horizontal axis in the plot of each set of results is the range of $\Delta V$ values, and the vertical axis is the percent of time in which the value falls into that particular range. The error bars present on each bin are the standard deviation given by averaging the individual normalized distributions. 


\subsection{Giant Danios}

The typical length of a giant danio was used to choose $d_{s e p}$, in this case it was set to five centimeters [28]. The maximum allowable velocity was set to $30 \mathrm{~cm} / \mathrm{s}$. The topological sensing range was choosen to be three, seven or all nearest neighbors, and the metric range,

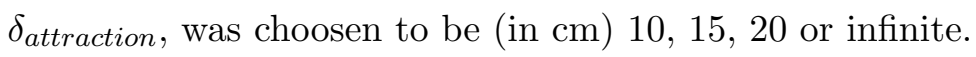

\subsubsection{Global Coordinates}

Constant Speed Maneuver

The constant speed maneuver in the global coordinate system was the simplest approach. The following plots were the average distributions of the norm of the difference between the optimal solution to the algorithm and the actual new velocity vector of the animal. The average has been normalized by the number of conflicts each biological species encountered. The bin size on each graph is $4 \mathrm{~cm} / \mathrm{s}$.

Two parameters were varied in these plots, $\delta_{\text {attraction }}$ and the number of influential nearest neighbors. The first set of graphs, Figures 5.1-5.15, fixed $\delta_{\text {attraction }}$ at infinity and varied the number of influential neighbors for all data sets. Each distribution has three subplots corresponding to the Beginning, Middle, and End (before, immediately after and longer past a food stimulus) segments of each data set. The data set $0-15$ (no trained fish, 15 untrained fish) did not have an End segment.

None of the results with this approach showed a strong correlation between the biological data and the algorithmic predictions. All distributions showed that the average $\Delta V$ for each fish was less $4 \mathrm{~cm} / \mathrm{s}$ less than $40 \%$ of the time. No significant difference between the distributions for the different numbers of influential nearest neighbors was observed. The Middle segment, while the fish were actively searching for food, generally showed worse results than the Beginning and End segments. These results are consistent with the idea that the fish were less likely to care about colliding with one another while searching for food. 
Variable Speed Maneuver

The global coordinate variable speed maneuver results, Figures 5.16-5.33, indicated higher correlation than the global coordinate constant speed maneuver results. The $0-15$ data set plots, Figures 5.16-5.18, showed a much higher correlation than the constant speed maneuver. On average, $70 \%$ of the time $\Delta V$ was $4 \mathrm{~cm} / \mathrm{s}$ or less, compared to the constant speed maneuver which was approximately $30 \%$. The Middle segment also saw an improvement, from $20 \%$ for the constant speed to over $40 \%$ for the variable speed maneuver. Once again, the Middle segment showed poorer results in comparison to the Beginning segment. No significant change with the number of influential nearest neighbors was seen in the results when compared to the $\delta_{\text {attraction }}$ results. The $06-9$ data set distribution, shown in Figures 5.19-5.21, did not vary with the number of influential nearest neighbors. The End segment had the most improvement going from the $\Delta V$ being $4 \mathrm{~cm} / \mathrm{s}$ or less $30 \%$ to $80 \%$ of the time. One feature to note in these distributions is that the Beginning segment had the worst performance with the first bin at 30\%, compared to $40 \%$ for the Middle segment and $80 \%$ for the End segment. In all of the distributions, the End segment was always better than the Middle segment, and the Middle was always better than the Beginning segment. Potentially the fish learned not to run into each other over time, but further data would need to be analyzed to say anything concrete. The distribution of the 9-6 data set showed that $\Delta V$ was $4 \mathrm{~cm} / \mathrm{s}$ or less for the Beginning and End segment for between $60 \%$ and $70 \%$ of all interactions, with the exception of the End segment that corresponded to having only three influential nearest neighbors. These results were a significant improvement from the constant speed maneuver which had $\Delta V 4 \mathrm{~cm} / \mathrm{s}$ or less for less than $40 \%$ of the interations. The middle segment still showed poor performance as expected. A slight increase in the percent of $\Delta V$ values being less than four $\mathrm{cm} / \mathrm{s}$ was found, for the 12-3 data set, when increasing the number of influential nearest neighbors. More change occured going from three to seven neighbors than going from seven to all neighbors. The Beginning and End segments showed that the $\Delta V$ was less than $4 \mathrm{~cm} / \mathrm{s}$ from just less than $70 \%$ of the time to just below $80 \%$ of the time. The constant speed maneuver correlation was much lower, below $40 \%$ of the time. When varying $\delta_{\text {attraction }}$ and keeping the number of influential nearest 
neighbors at seven for the 12-3 data set, one can see that as the $\delta_{\text {attraction }}$ decreased so did the performance of the algorithm (Figures 5.28-5.30). The Beginning and End segments of the 15-0 data set (Figures 5.31-5.33) showed promising results with $\Delta V$ values of less than $4 \mathrm{~cm} / \mathrm{s}$ just less than $80 \%$ or the time for three influential nearest neighbors and just greater than $80 \%$ of the time for seven influential nearest neighbors and the maximum number of neighbors. The Mid segment showed poor performance, with $\Delta V$ being less the $4 \mathrm{~cm} / \mathrm{s}$ close to $20 \%$ of the time indicating that all of the fish in the 15-0 data set had a high correlation between the predicted optimal velocity and the actual velocity vectors. Another indication of good performance was that the small standard deviation on the first bin. The $\Delta V$ values greater than $8 \mathrm{~cm} / \mathrm{s}$ occured less than $10 \%$ of the time. For the 15-0 data set, when keeping the number of influential nearest neighbors at seven and changing the maximum sensing distance, $\delta_{\text {attraction }}$, one can see that the correlation decreased as $\delta_{\text {atrraction }}$ gets smaller (Figure 5.34-5.36). When comparing the data sets to one another, the groups with more trained fish appeared to do a better job of moving themselves out of conflict according to the analytical model of optimal deconfliction than did the groups with more untrained fish. When the number of trained fish was greater then the number of untrained fish, the collision cone algorithm appears highly correlated for the variable velocity global coordinate system maneuver.

\subsubsection{Body Coordinates}

The next set of results consider the giant Danio data using the body-fixed coordinate frame planar data projection. A higher correlation, in comparison to the global coordinate projection, between the predicted and actual velocities is expected.

\section{Constant Speed Maneuver}

The 0-15 data set showed that only around $20 \%$ of the time will a fish have a $\Delta V$ value less than $4 \mathrm{~cm} / \mathrm{s}$. These correlations were significantly lower than in the case of the global coordinate sstem. Similarly, the 06-9 data set used a constant speed maneuver in body coordinates has $\Delta V$ value less than $4 \mathrm{~cm} / \mathrm{s}$ a maximum of $30 \%$ of the time (Figures $5.40-5.42$ ). 
The $\Delta V$ results for the body-fixed coordinate system with constant speed maneuvering were also noticeably less than the global coordinate system with constant speed maneuvering. The 9-6 data set had a $\Delta V$ that was less than $4 \mathrm{~cm} / \mathrm{s}$ barely $20 \%$ of the time for any given parameters (Figures 5.43-5.45). The 12-3 data set for the fixed-body coordinate constant speed maneuver results again showed a low correlation; at best, during the End segments, the $\Delta V$ less than $4 \mathrm{~cm} / \mathrm{s}$ was $40 \%$ of the time (Figures 5.46-5.48). When setting the number of influential nearest neighbors to all and changing the $\delta_{\text {attraction }}$, the distributions showed even poorer results than the global coordinate constant speed maneuver with the same parameter constraints (Figures 5.52-5.54). For all data sets, the constant speed maneuver calculated in body coordinates generated worse results than the global coordinate frame constant speed results. The values of $\Delta V$ that were less than $4 \mathrm{~cm} / \mathrm{s}$ occured from less than $20 \%$ to just over $30 \%$ of the time.

\section{Variable Speed Maneuver}

The following plots, Figures 5.55-5.69, were the $\Delta V$ distributions for the variable speed maneuver in body coordinates. To determine which, if any, of the topological metrics the giant Danio used, the $\delta_{\text {attraction }}$ was fixed at infinity while the number of influential nearest neighbors was varied. The $\Delta V$ distribution for the $0-15$ data set Figures 5.55-5.69, once again had worse results than the global coordinate system results with the average $\Delta V$ being less than $4 \mathrm{~cm} / \mathrm{s}$ less than $40 \%$ of the time. The results for three influential nearest neighbors, Figure 5.55, showed slightly better performance, about a $10 \%$ higher in the less than $4 \mathrm{~cm} / \mathrm{s}$ bin, than the seven or all influential nearest neighbors (Figures 5.56-5.57). The 06-9 data set distribution for the variable speed maneuver in body coordinates did not even reach $40 \%$ for the $\Delta V$ being less than $4 \mathrm{~cm} / \mathrm{s}$ (Figures 5.58-5.60). The 9-6 data set showed that the average $\Delta V$ was more often $4-8 \mathrm{~cm} / \mathrm{s}$ than less than $4 \mathrm{~cm} / \mathrm{s}$, and being within those values not more than $30 \%$ of the time (Figures 5.61-5.63). For the 12-3 data set, Figures 5.64-5.66, the average $\Delta V$ distribution showed that during the End segment for seven and for all influential nearest neighbors just over $40 \%$ of the time, the $\Delta V$ value was less than $4 \mathrm{~cm} / \mathrm{s}$. For the remaining Beginning and End segments, the $\Delta V$ value was less than 4 
$\mathrm{cm} / \mathrm{s}$ less than $40 \%$ of the time. The $15-0$ data set completes the variable speed maneuver in body coordinate analysis for schooling fish. The distribution showed that the average $\Delta V$ value was less than $4 \mathrm{~cm} / \mathrm{s}$ for at most $40 \%$ of the time. Overall, and consistently, the variable speed maneuver in body coordinates produced low correlation for all data sets. Results indicate that giant Danios are not performing deconfliction using collision cones in this way.

\subsubsection{Schooling Fish Discussion}

The algorithm aligns best with what the schooling giant danios were doing when using a global coordinate frame and a variable speed maneuver. The body coordinate frame, for either maneuver, did not show any promising results. The fixed-body frame coordinate system may have produced a lower correlation than the global coordinate system due to how the giant Danios view their surroundings through the water. In the future, a third coordinate system, based on the vision of a fish, could be used in an attempt to get higher coorelated results.
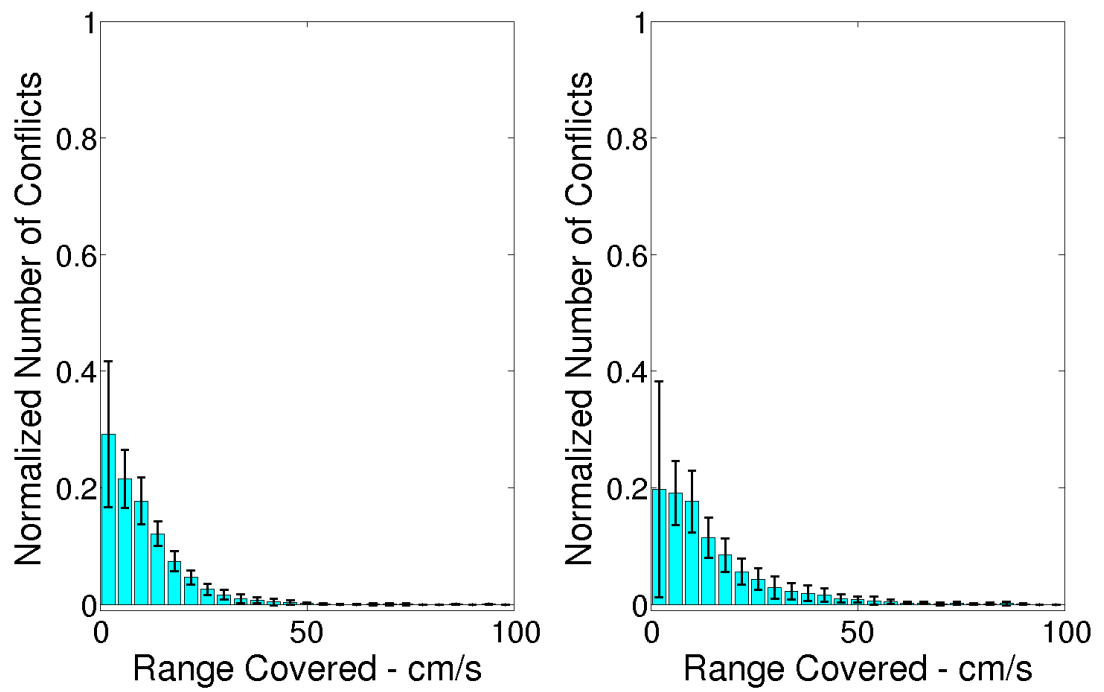

Figure 5.1: The $\Delta V$ distribution for three influential neighbors and $\delta_{\text {attraction }}=$ infinity for the 0-15 giant danio data set under the global coordinate system with constant speed maneuver. 

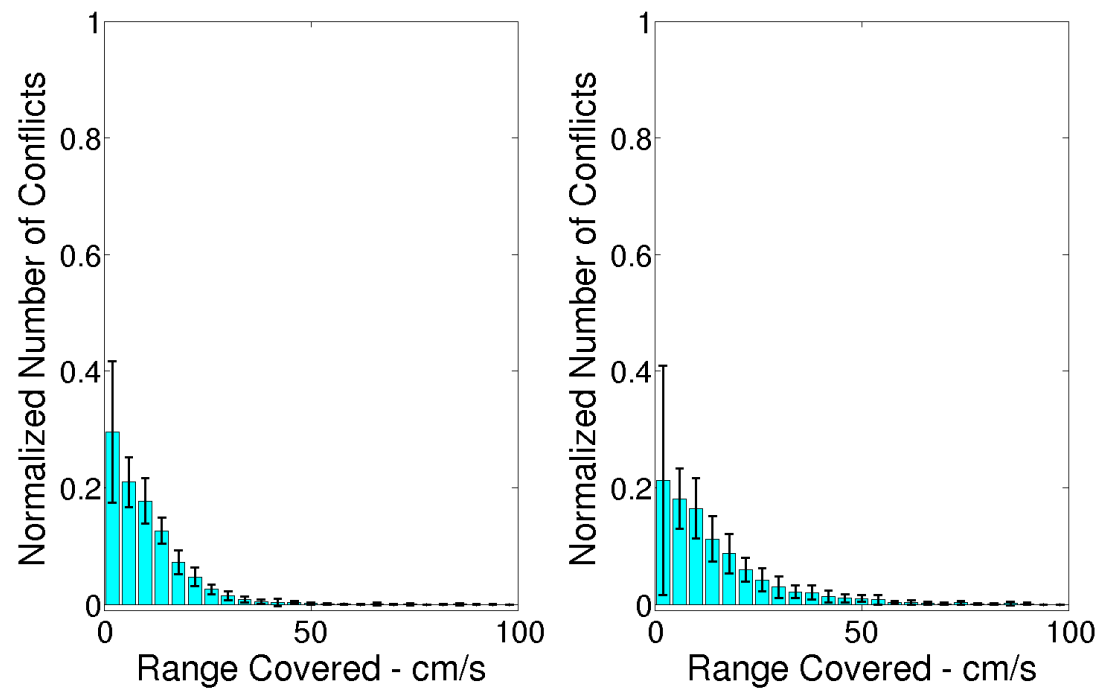

Figure 5.2: The $\Delta V$ distribution for seven influential neighbors and $\delta_{\text {attraction }}=$ infinity for the 0-15 giant danio data set under the global coordinate system with the constant speed maneuver.
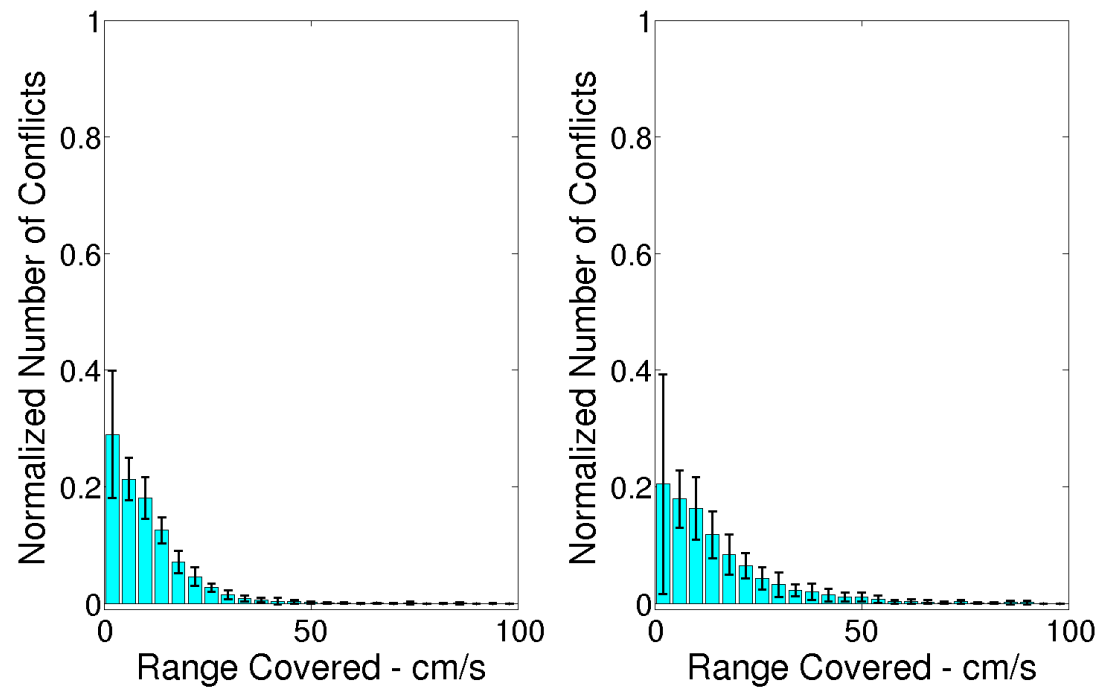

Figure 5.3: The $\Delta V$ distribution for the maximum number of influential neighbors and $\delta_{\text {attraction }}=$ infinity for the $0-15$ giant danio data set under the global coordinate system with the constant speed maneuver. 


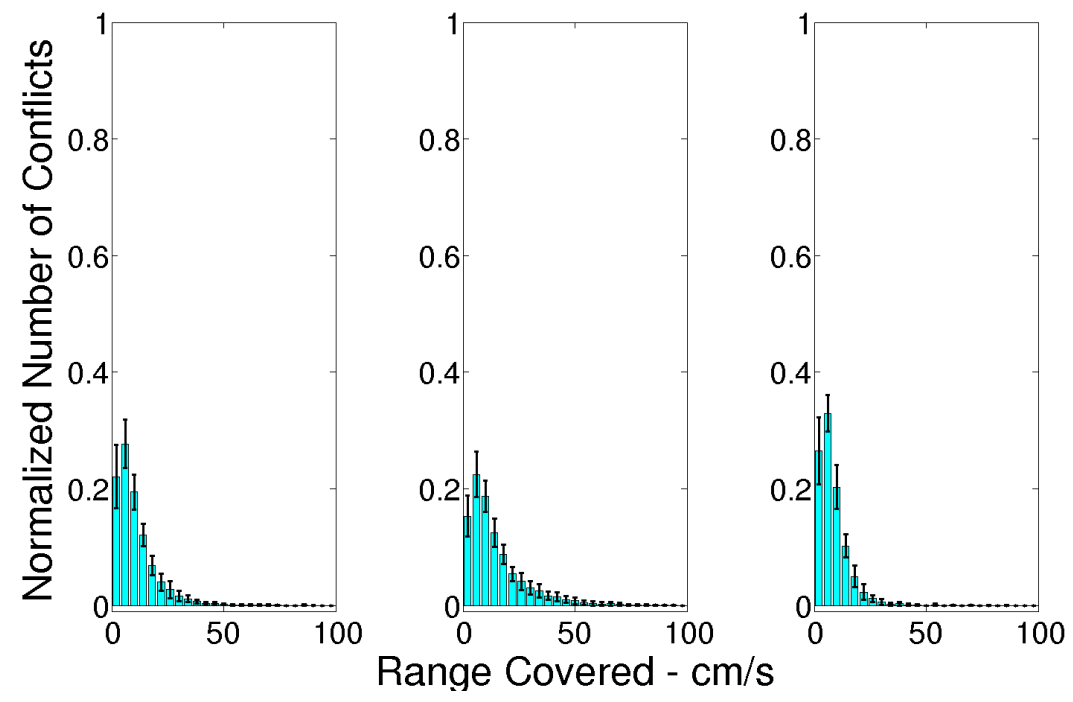

Figure 5.4: The $\Delta V$ distribution for three influential neighbors and $\delta_{\text {attraction }}=$ infinity for the 6-9 giant danio data set under the global coordinate system with the constant speed maneuver.
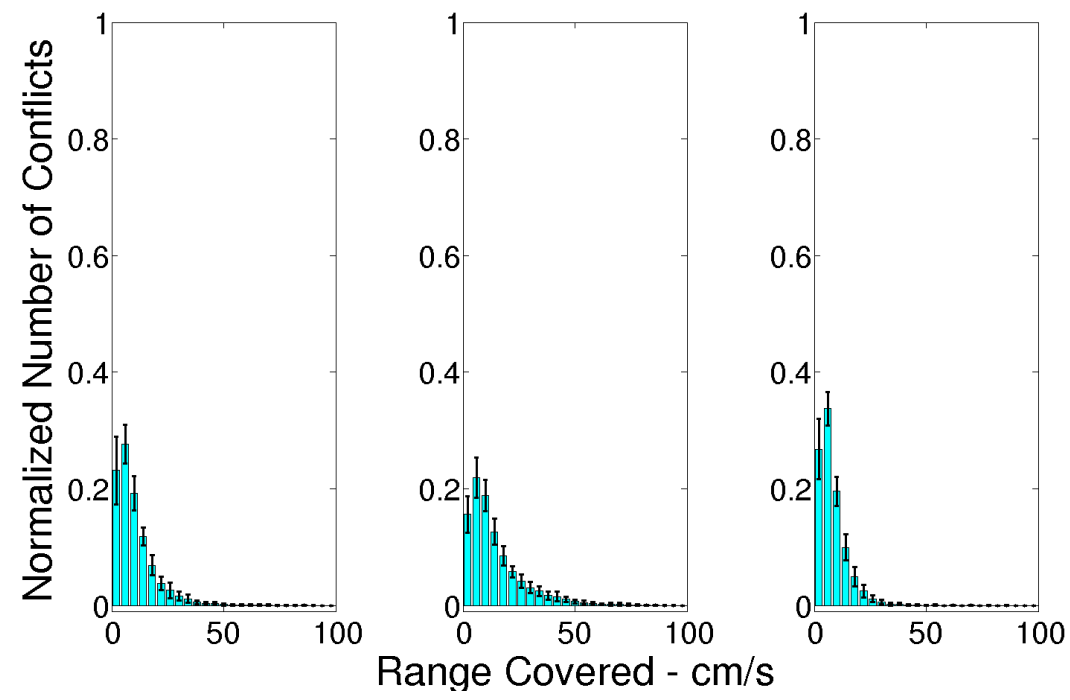

Figure 5.5: The $\Delta V$ distribution for seven influential neighbors and $\delta_{\text {attraction }}=$ infinity for the 6-9 giant danio data set under the global coordinate system with the constant speed maneuver. 


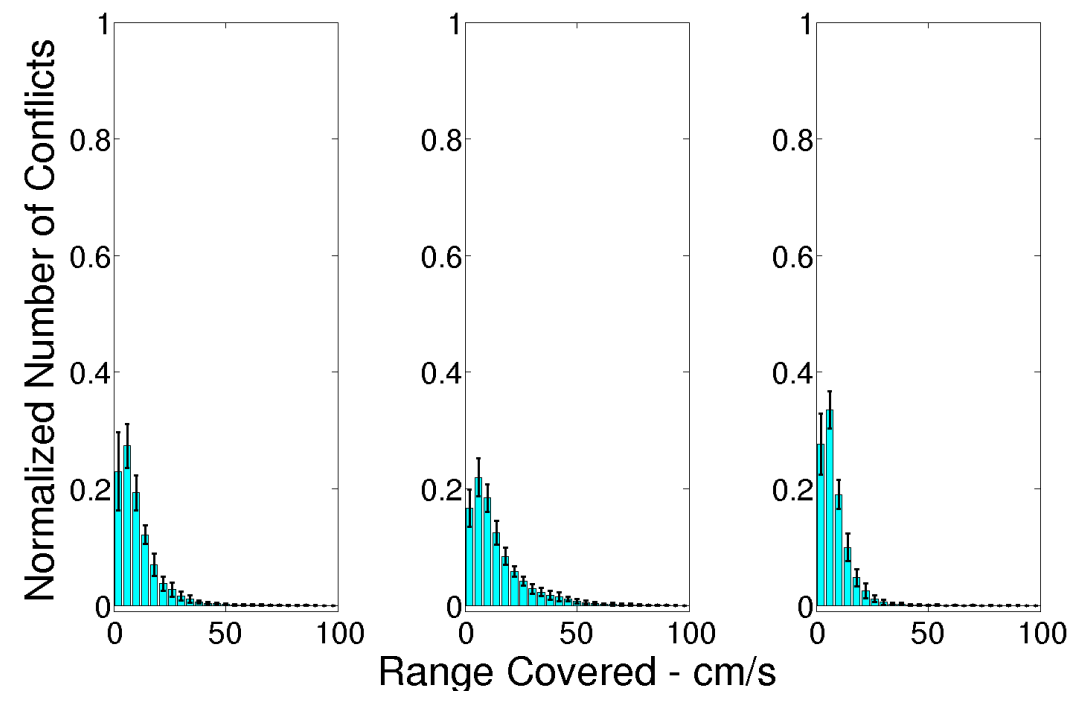

Figure 5.6: The $\Delta V$ distribution for the maximum number of influential neighbors and $\delta_{\text {attraction }}=$ infinity for the 6-9 giant danio data set under the global coordinate system with the constant speed maneuver.
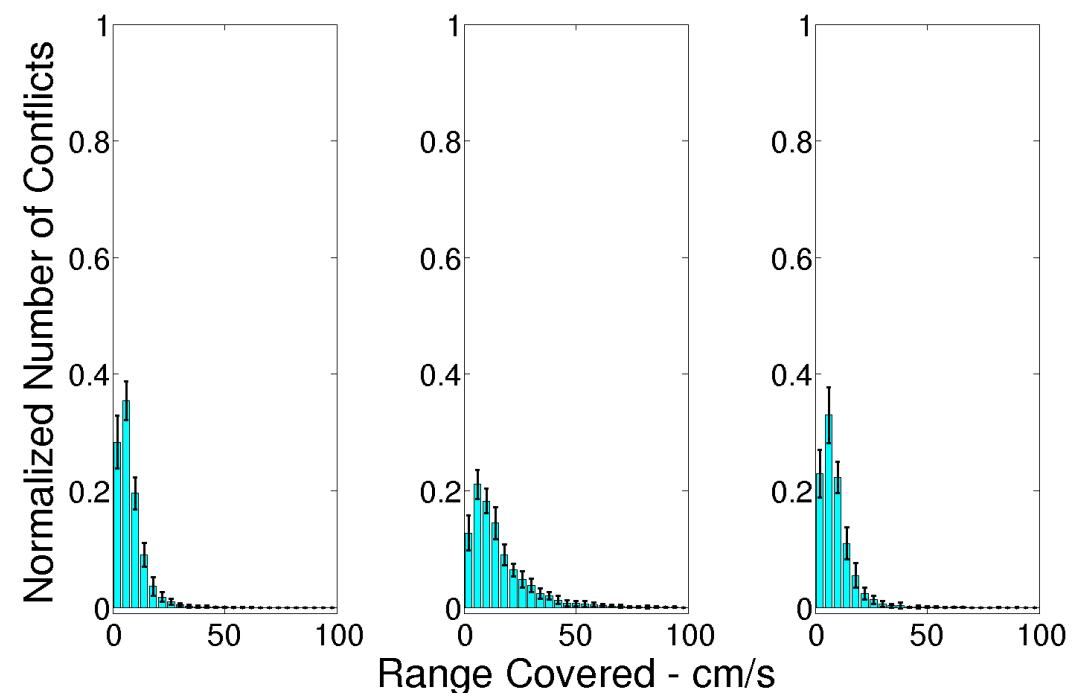

Figure 5.7: The $\Delta V$ distribution for three influential neighbors and $\delta_{\text {attraction }}=$ infinity for the 9-6 giant danio data set under the global coordinate system with the constant speed maneuver. 

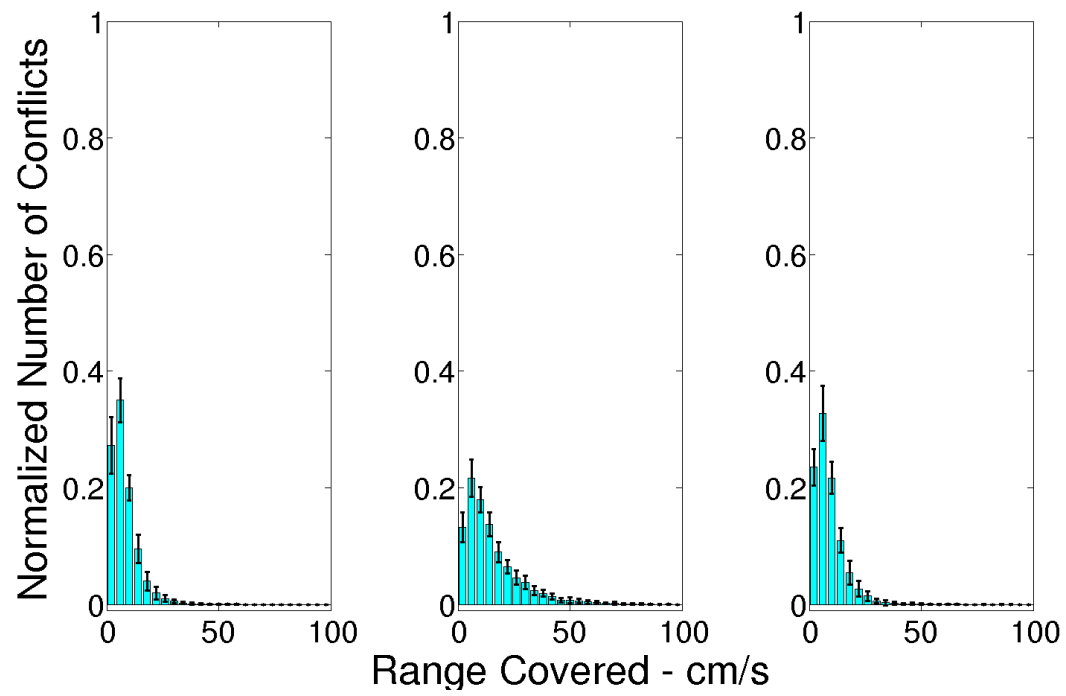

Figure 5.8: The $\Delta V$ distribution for seven influential neighbors and $\delta_{\text {attraction }}=$ infinity for the 9-6 giant danio data set under the global coordinate system with the constant speed maneuver.
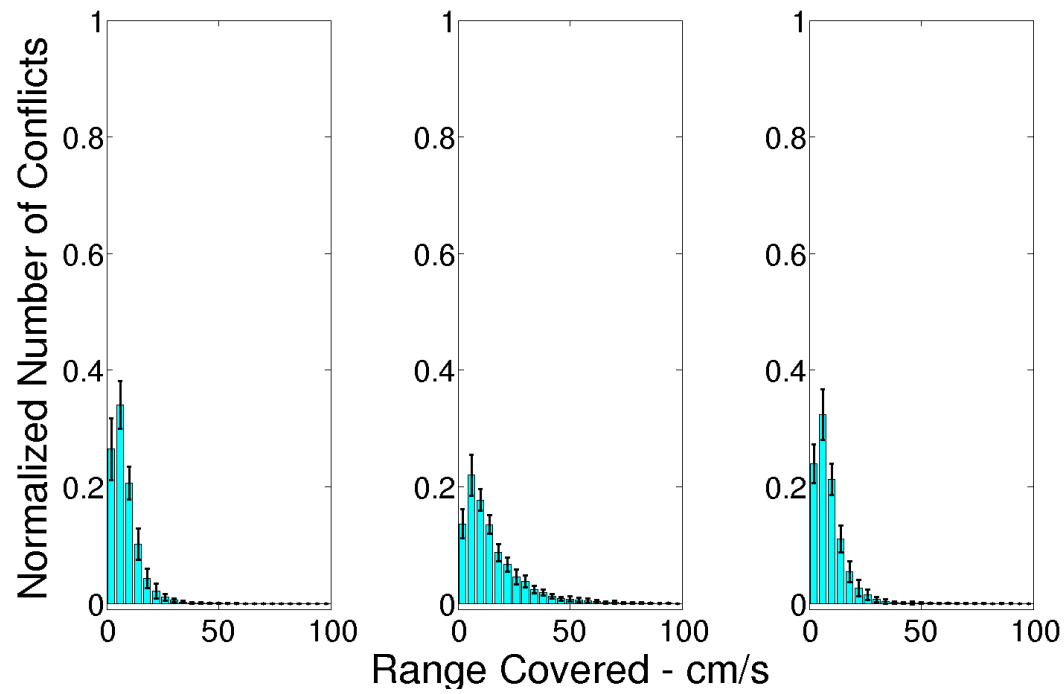

Figure 5.9: The $\Delta V$ distribution for the maximum number of influential neighbors and $\delta_{\text {attraction }}=$ infinity for the 9-6 giant danio data set under the global coordinate system with the constant speed maneuver. 

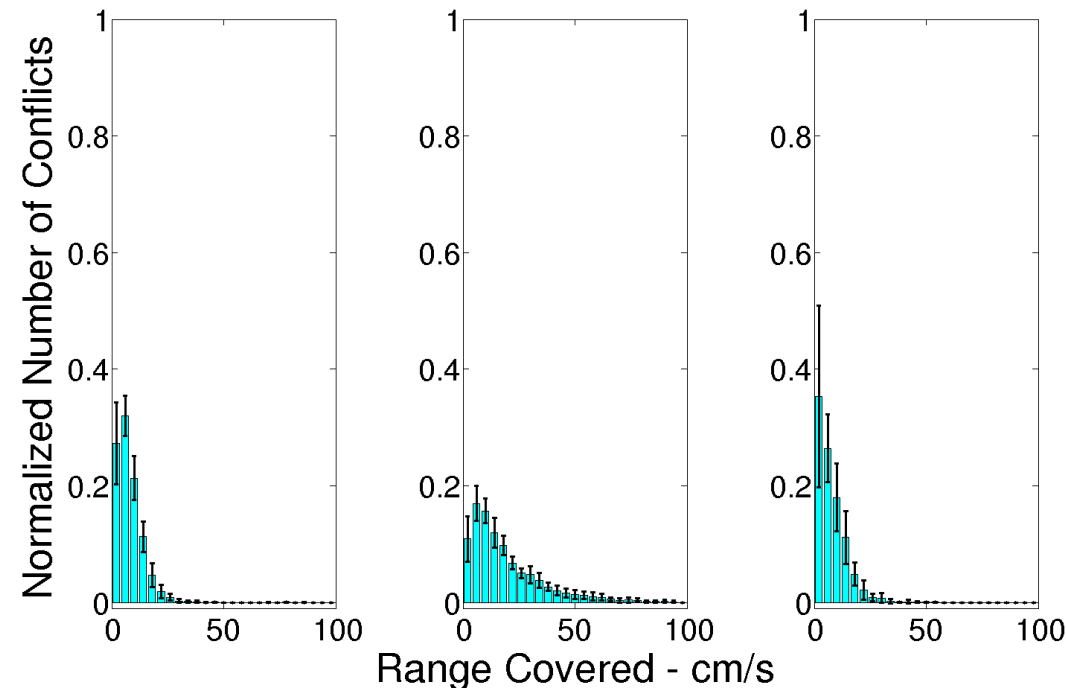

Figure 5.10: The $\Delta V$ distribution for three influential neighbors and $\delta_{\text {attraction }}=$ infinity for the 12-3 giant danio data set under the global coordinate system with the constant speed maneuver..

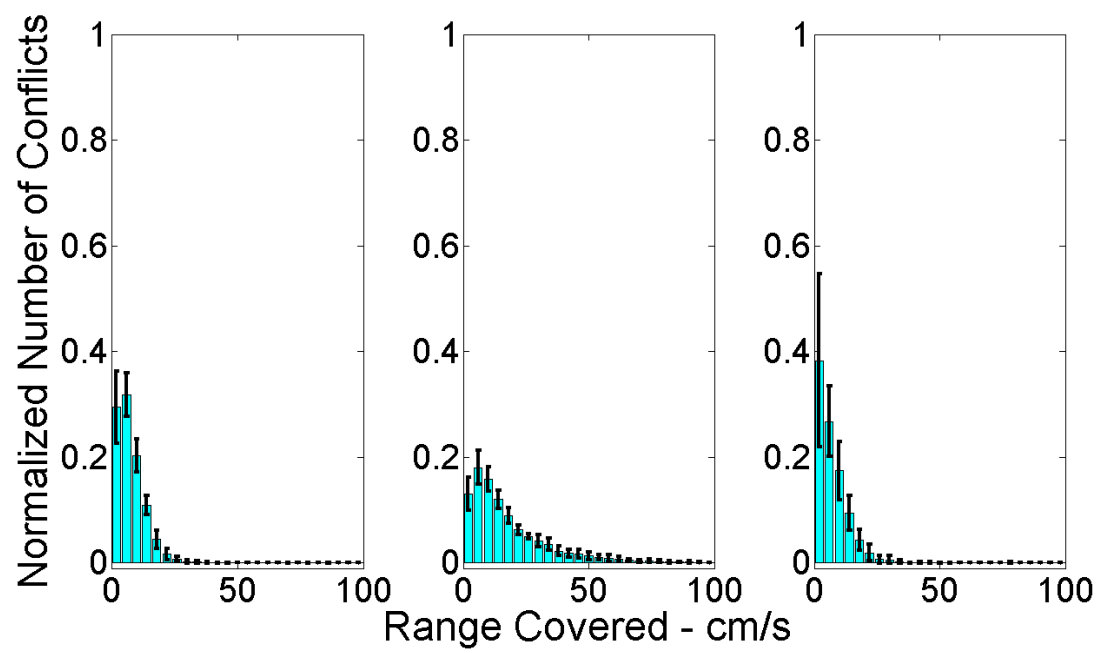

Figure 5.11: The $\Delta V$ distribution for seven influential neighbors and $\delta_{\text {attraction }}=$ infinity for the 12-3 giant danio data set under the global coordinate system with the constant speed maneuver. 

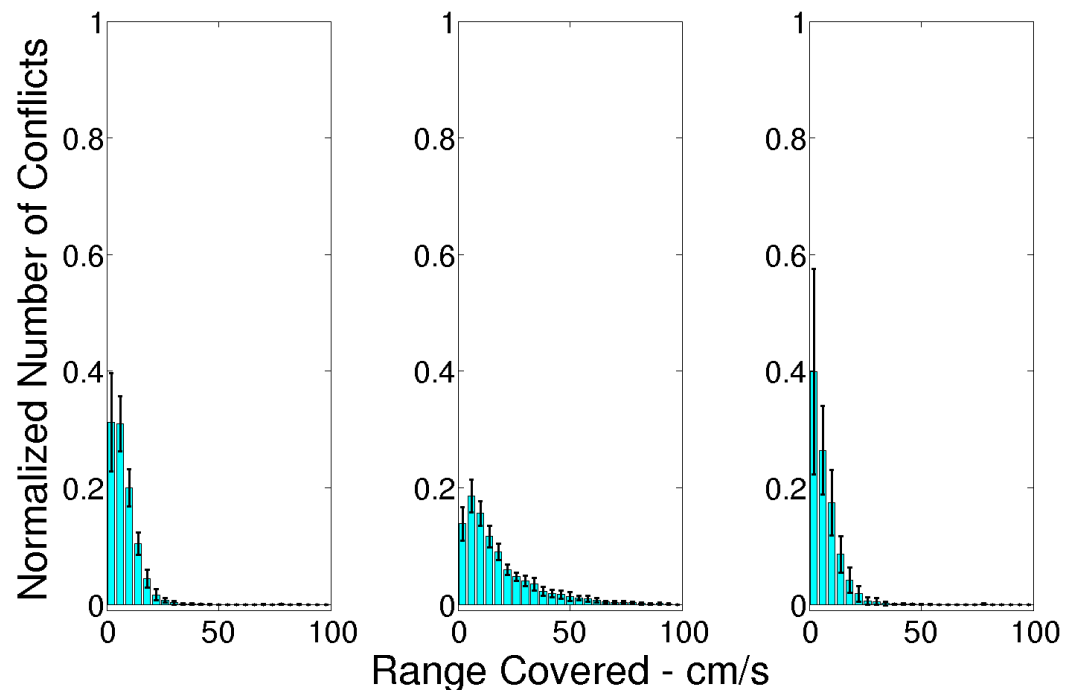

Figure 5.12: The $\Delta V$ distribution for the maximum number of influential neighbors and $\delta_{\text {attraction }}=$ infinity for the 12-3 giant danio data set under the global coordinate system with the constant speed maneuver.
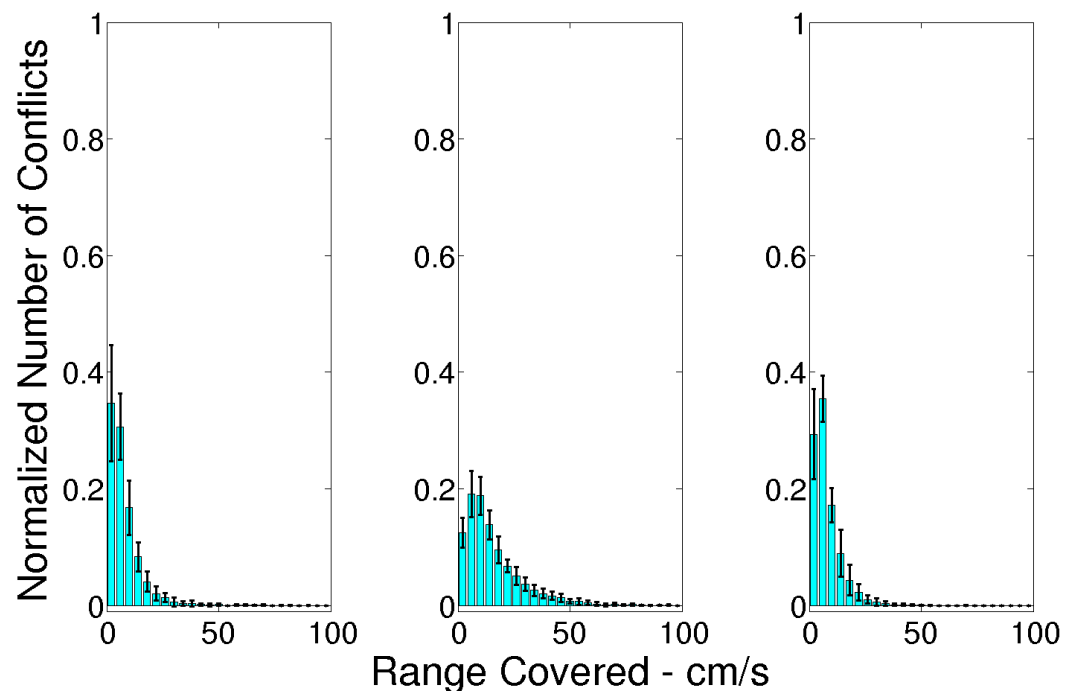

Figure 5.13: The $\Delta V$ distribution for three influential neighbors and $\delta_{\text {attraction }}=$ infinity for the 15-0 giant danio data set under the global coordinate system with the constant speed maneuver. 

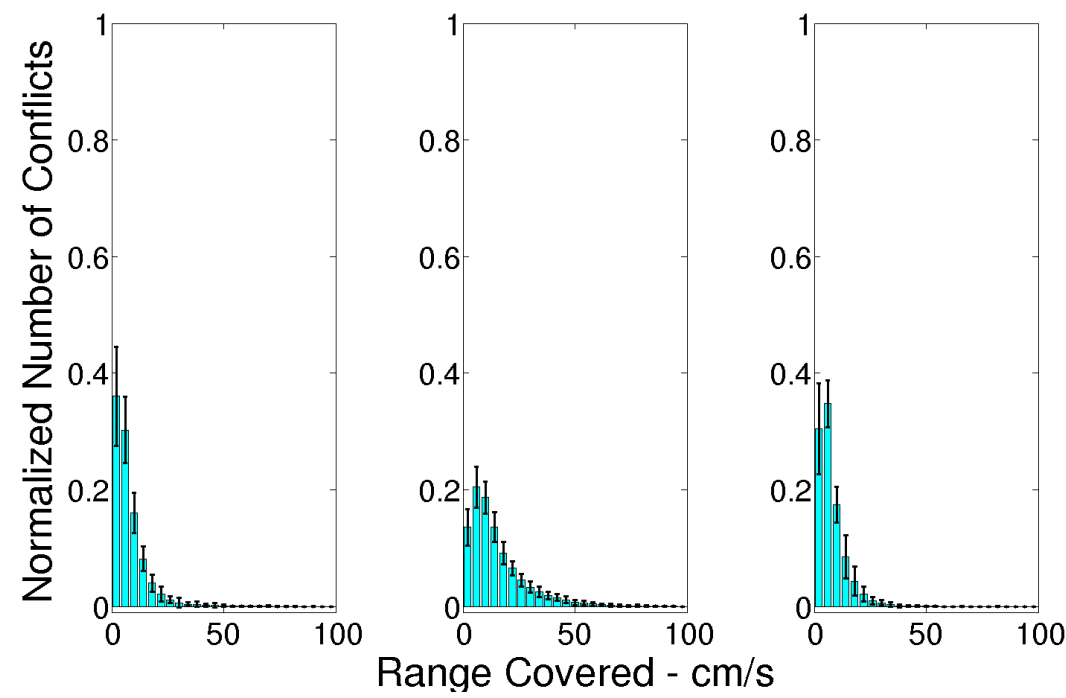

Figure 5.14: The $\Delta V$ distribution for seven influential neighbors and $\delta_{\text {attraction }}=$ infinity for the 15-0 giant danio data set under the global coordinate system with the constant speed maneuver.
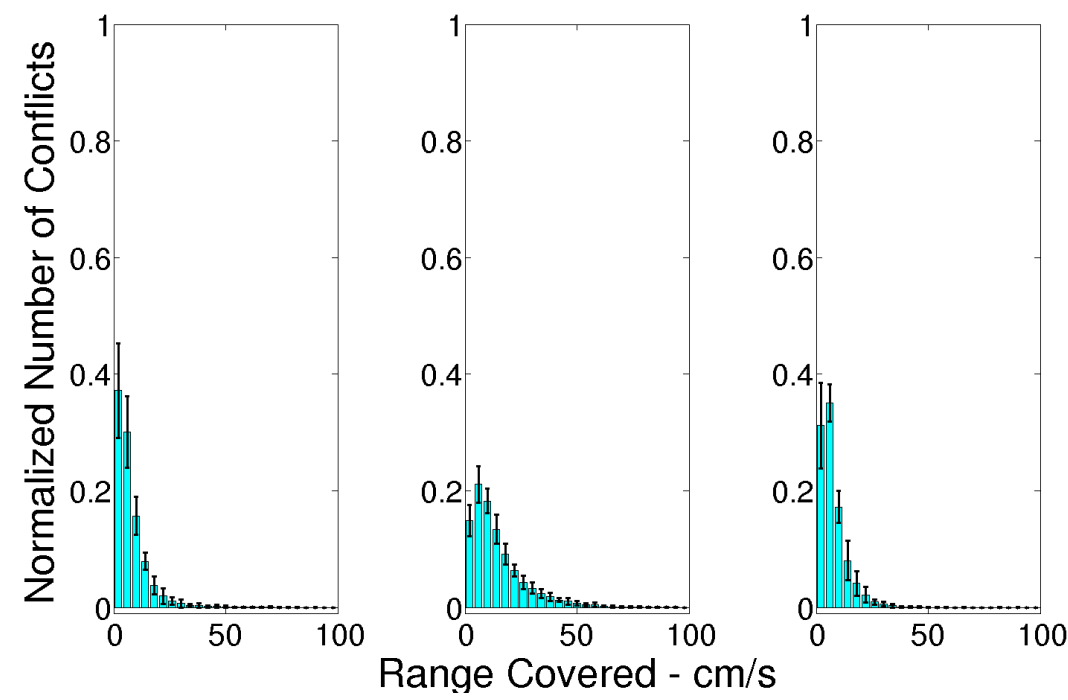

Figure 5.15: The $\Delta V$ distribution for the maximum number of influential neighbors and $\delta_{\text {attraction }}=$ infinity for the $15-0$ giant danio data set under the global coordinate system with the constant speed maneuver. 

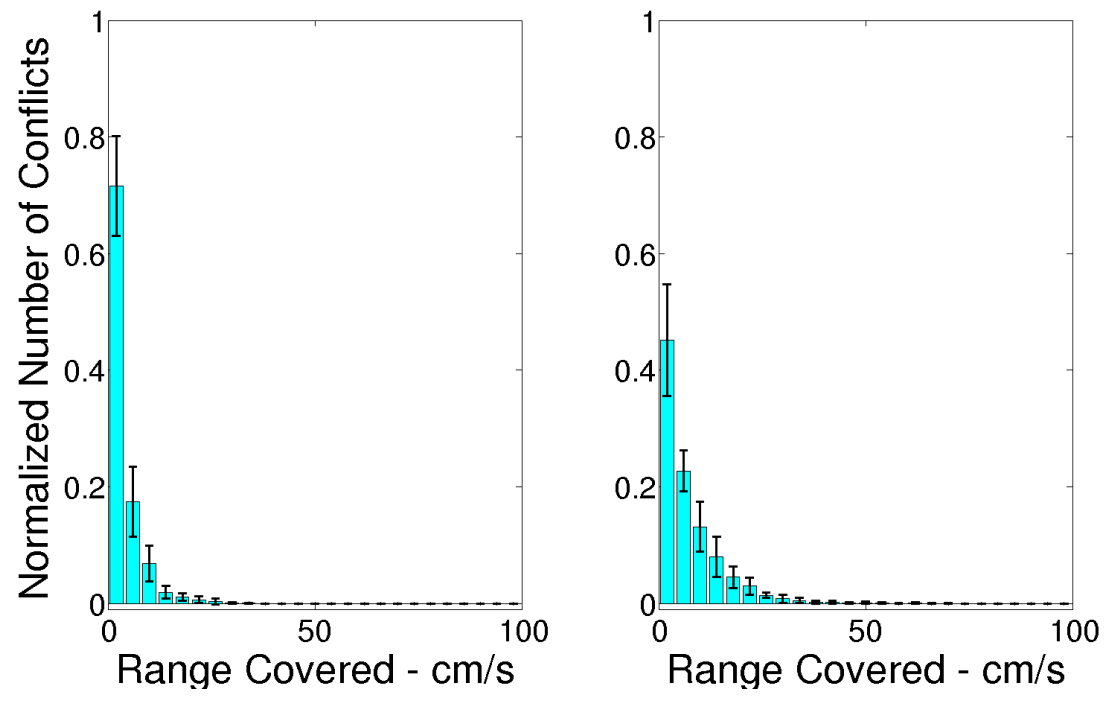

Figure 5.16: The $\Delta V$ distribution for three influential neighbors and $\delta_{\text {attraction }}=$ infinity for the 0-15 giant danio data set under the global coordinate system with the variable speed maneuver. 

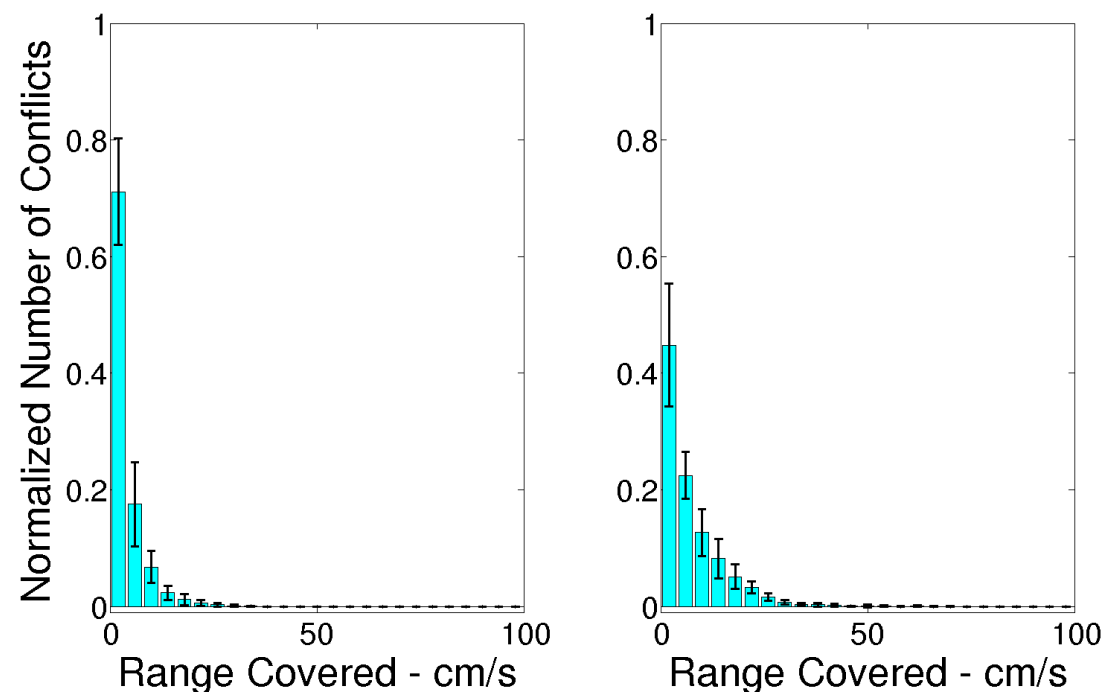

Figure 5.17: The $\Delta V$ distribution for seven influential neighbors and $\delta_{\text {attraction }}=$ infinity for the 0-15 giant danio data set under the global coordinate system with the variable speed maneuver.
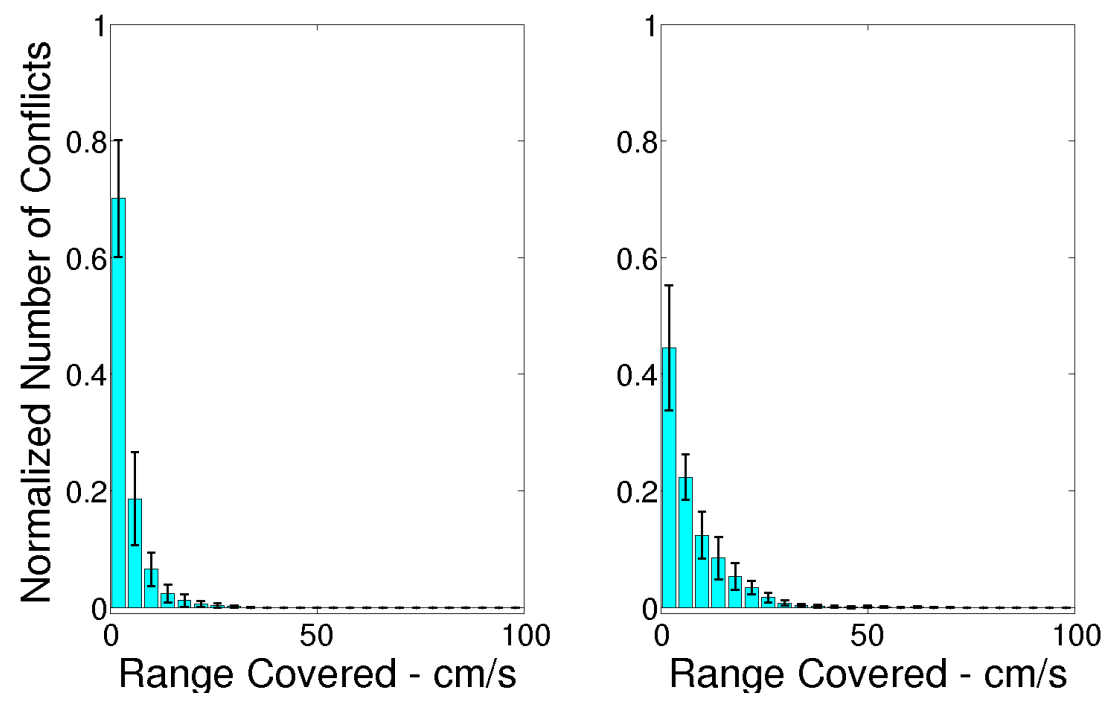

Figure 5.18: The $\Delta V$ distribution for the maximum number of influential neighbors and $\delta_{\text {attraction }}=$ infinity for the $0-15$ giant danio data set under the global coordinate system with the variable speed maneuver. 

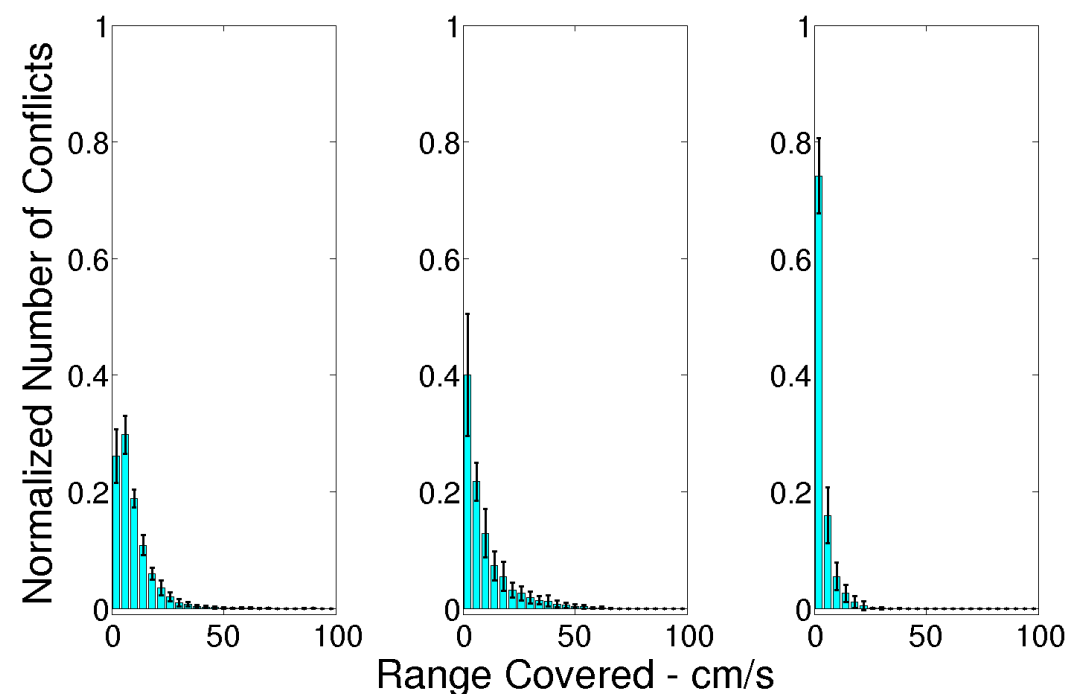

Figure 5.19: The $\Delta V$ distribution for three influential neighbors and $\delta_{\text {attraction }}=$ infinity for the 6-9 giant danio data set under the global coordinate system with the variable speed maneuver.
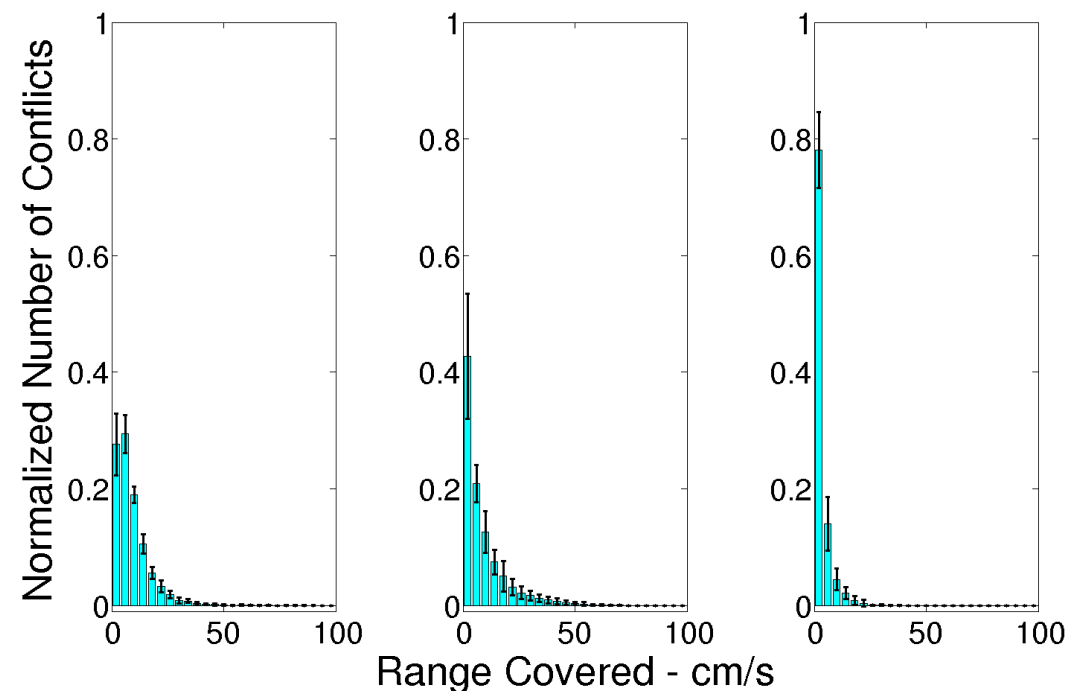

Figure 5.20: The $\Delta V$ distribution for seven influential neighbors and $\delta_{\text {attraction }}=$ infinity for the 6-9 giant danio data set under the global coordinate system with the variable speed maneuver. 


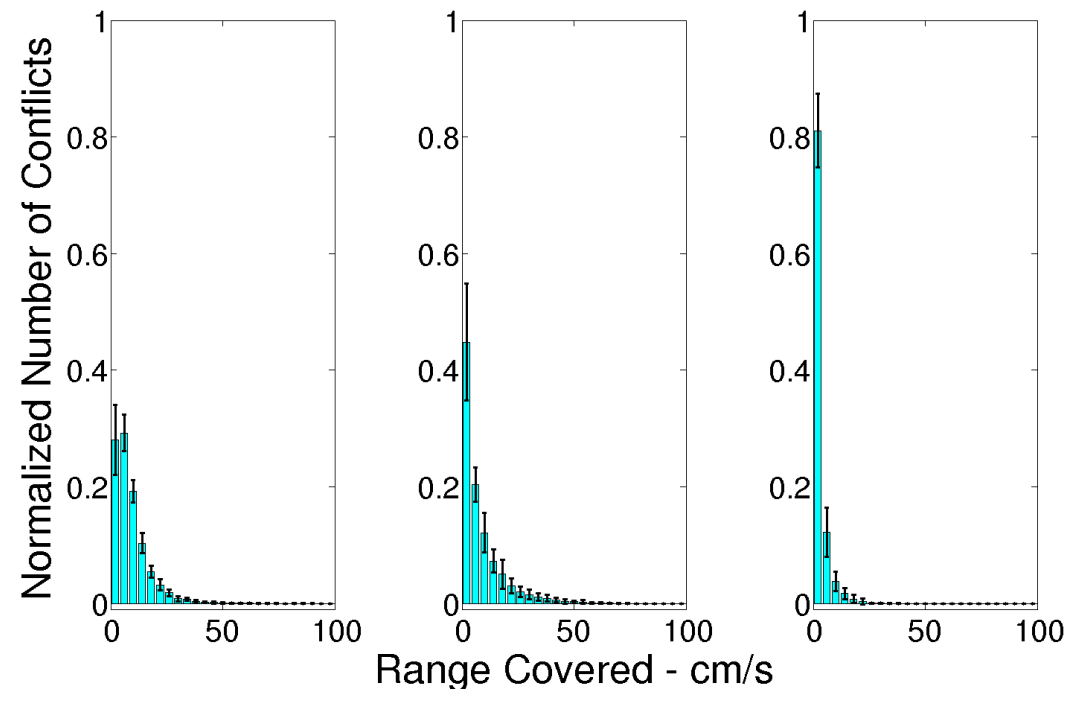

Figure 5.21: The $\Delta V$ distribution forthe maximum number of influential neighbors and $\delta_{\text {attraction }}=$ infinity for the 6-9 giant danio data set under the global coordinate system with the variable speed maneuver.
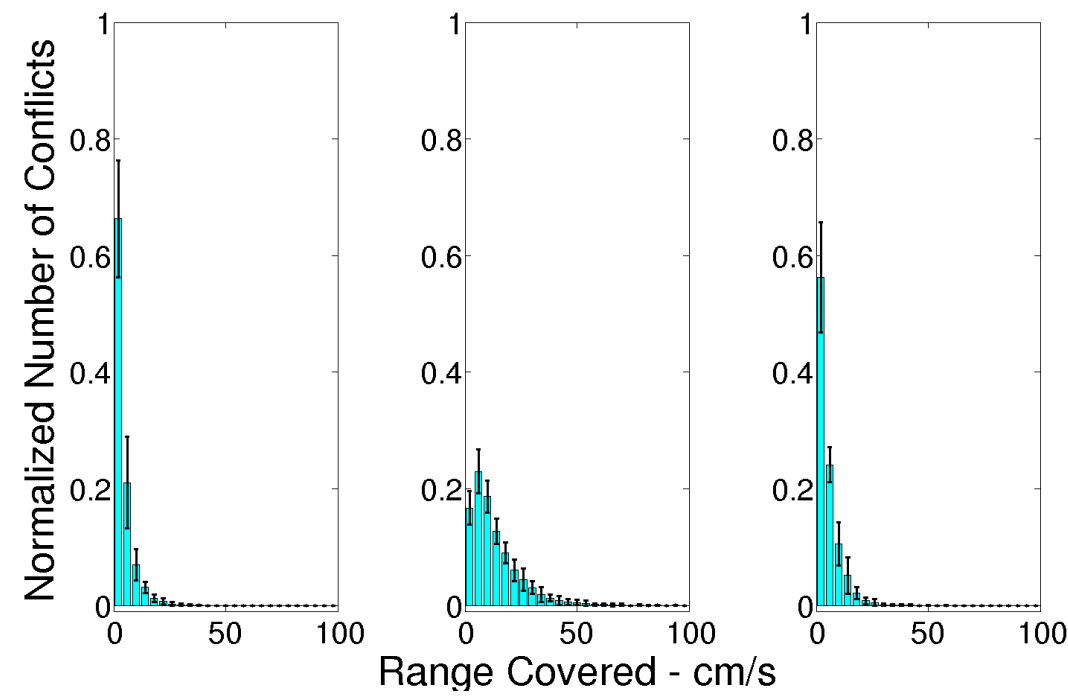

Figure 5.22: The $\Delta V$ distribution for three influential neighbors and $\delta_{\text {attraction }}=$ infinity for the 9-6 giant danio data set under the global coordinate system with the variable speed maneuver. 

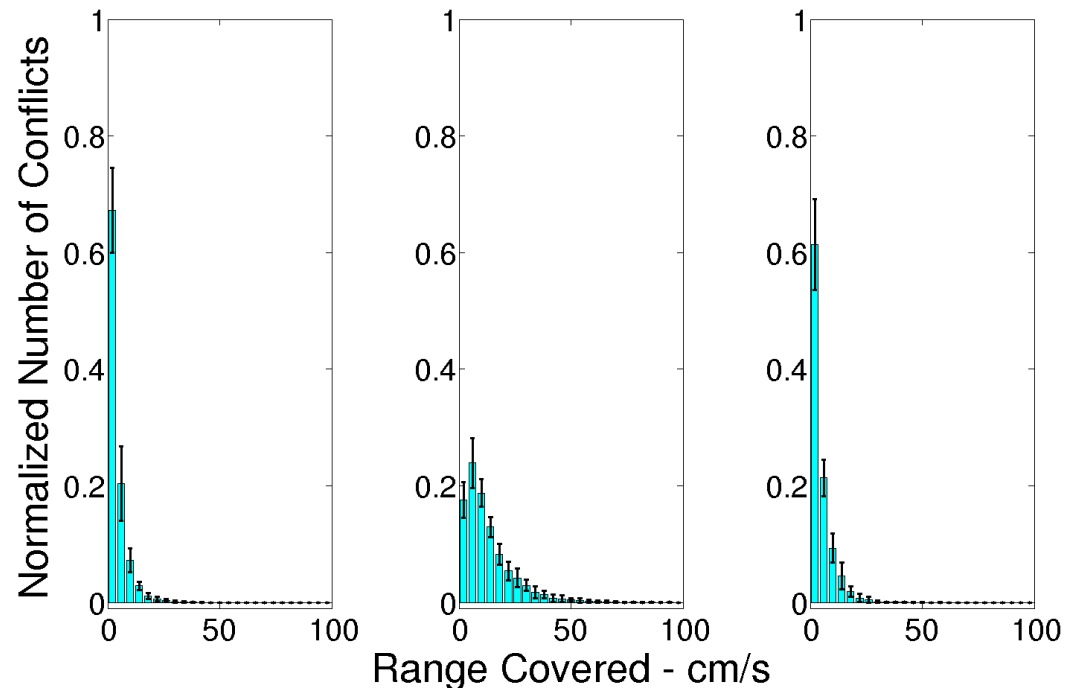

Figure 5.23: The $\Delta V$ distribution for seven influential neighbors and $\delta_{\text {attraction }}=$ infinity for the 9-6 giant danio data set under the global coordinate system with the variable speed maneuver.
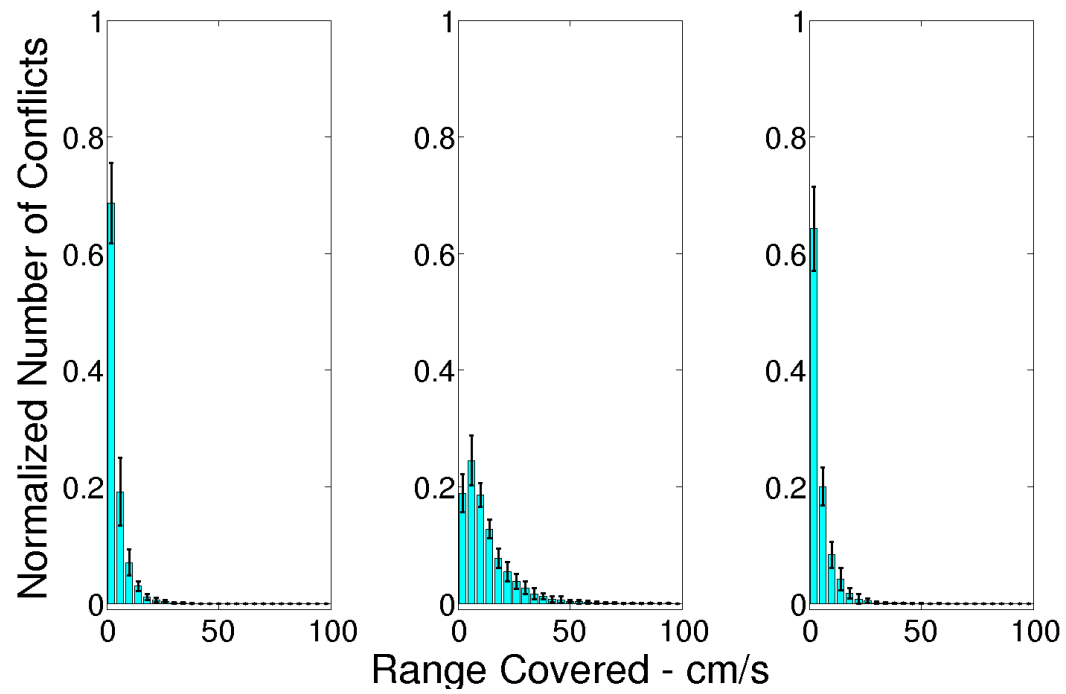

Figure 5.24: The $\Delta V$ distribution for the maximum number of influential neighbors and $\delta_{\text {attraction }}=$ infinity for the 9-6 giant danio data set under the global coordinate system with the variable speed maneuver. 


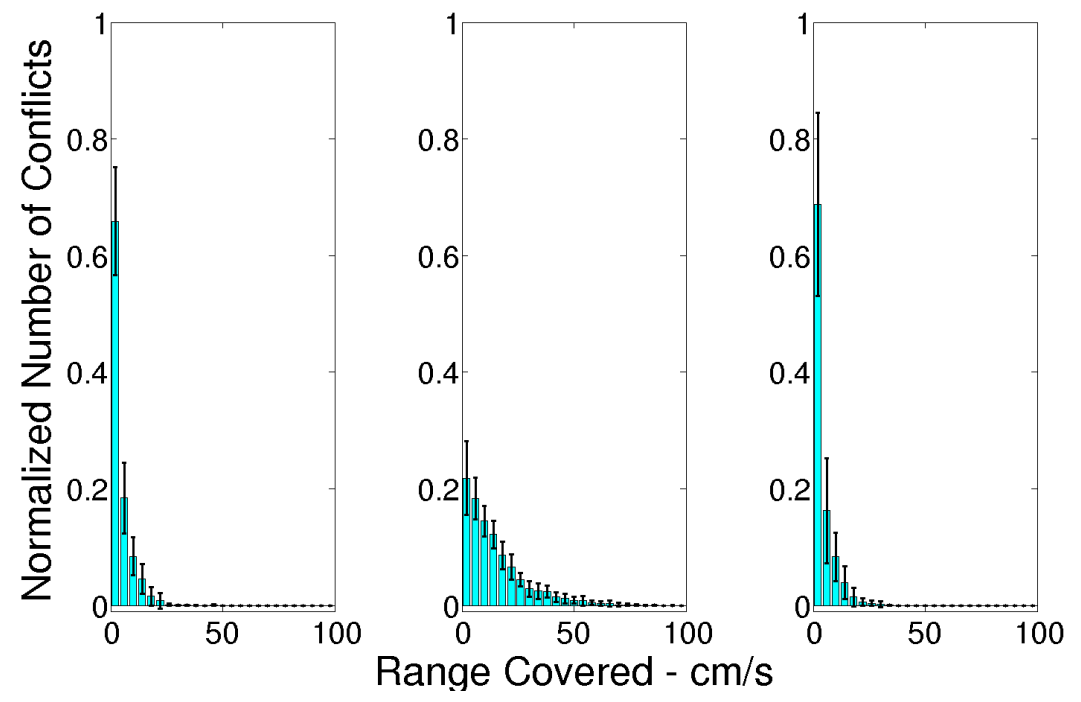

Figure 5.25: The $\Delta V$ distribution for three influential neighbors and $\delta_{\text {attraction }}=$ infinity for the 12-3 giant danio data set under the global coordinate system with the variable speed maneuver.
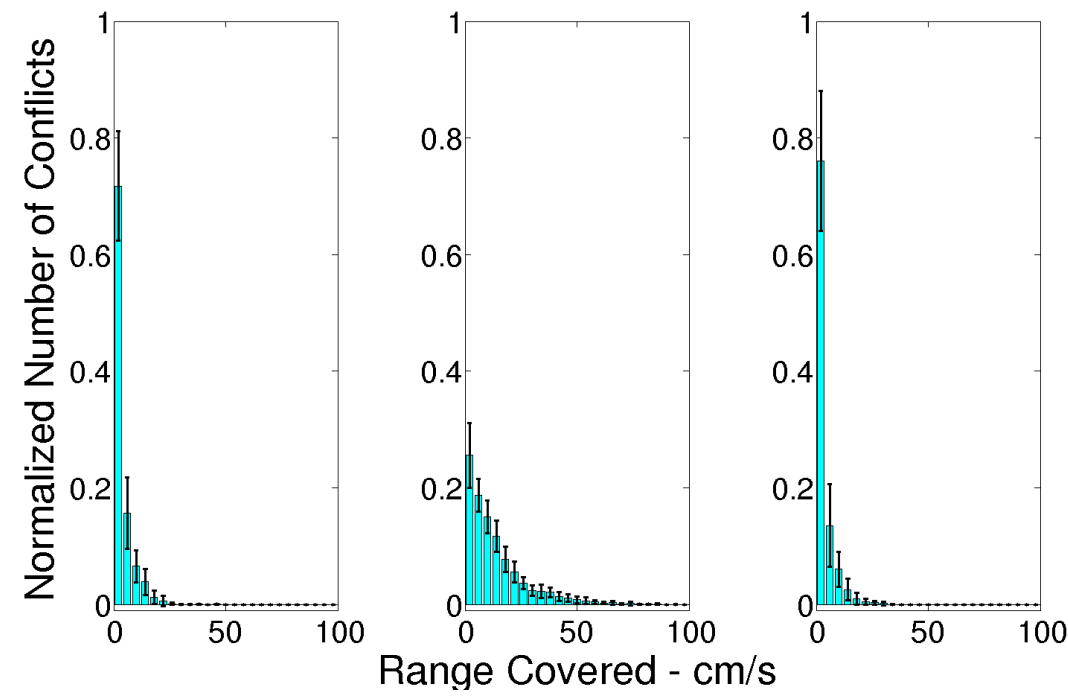

Figure 5.26: The $\Delta V$ distribution for seven influential neighbors and $\delta_{\text {attraction }}=$ infinity for the 12-3 giant danio data set under the global coordinate system with the variable speed maneuver. 

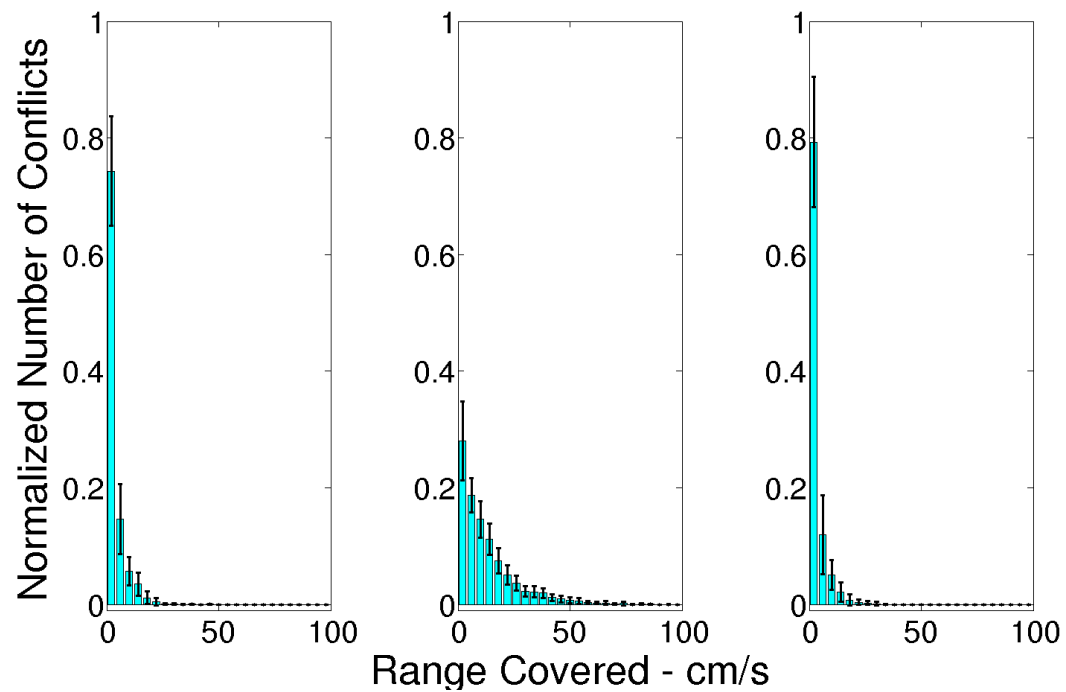

Figure 5.27: The $\Delta V$ distribution for the maximum number of influential neighbors and $\delta_{\text {attraction }}=$ infinity for the 12-3 giant danio data set under the global coordinate system with the variable speed maneuver.
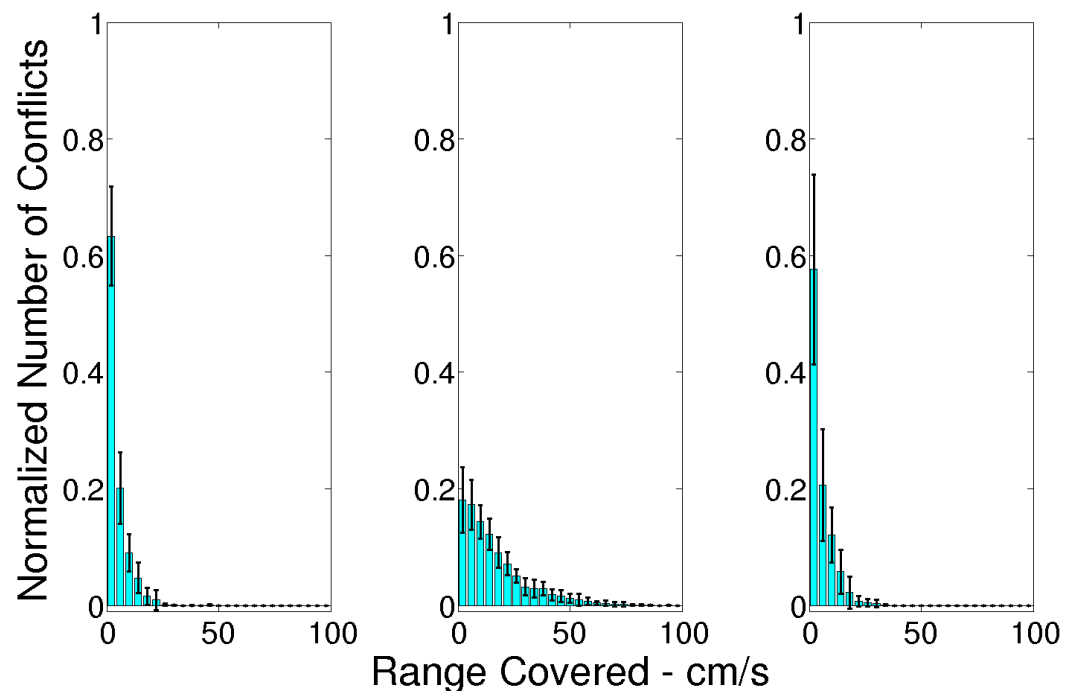

Figure 5.28: The $\Delta V$ distribution for seven influential neighbors and $\delta_{\text {attraction }}=20 \mathrm{~cm}$ for the 12-3 giant danio data set under the global coordinate system with the variable speed maneuver. 

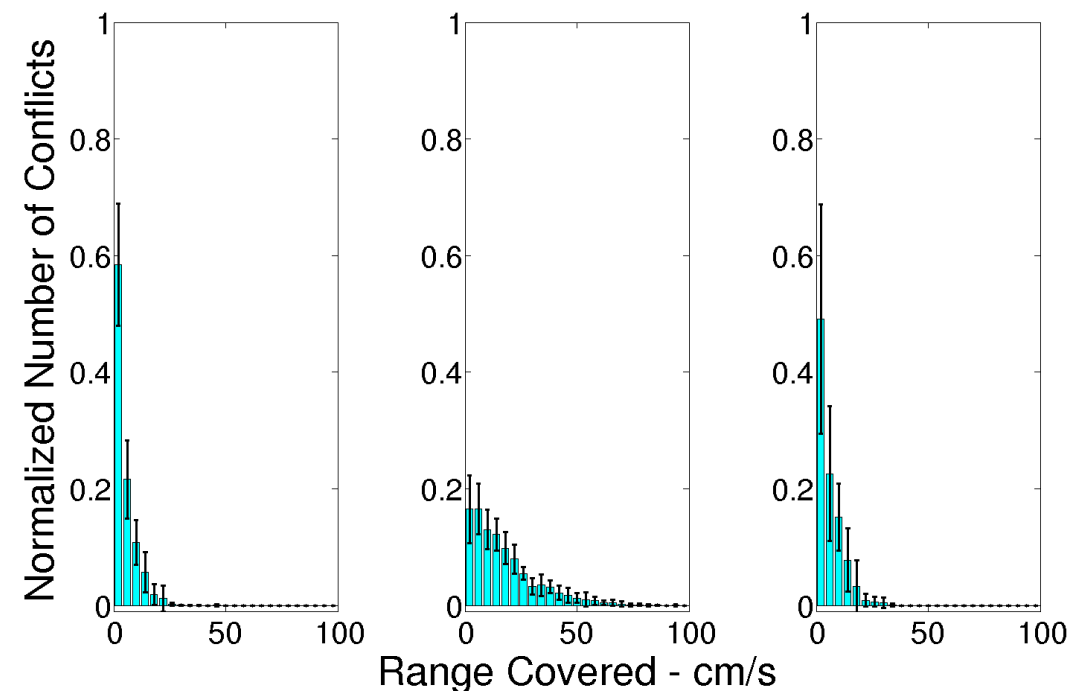

Figure 5.29: The $\Delta V$ distribution for seven influential neighbors and $\delta_{\text {attraction }}=15 \mathrm{~cm}$ for the 12-3 giant danio data set under the global coordinate system with the variable speed maneuver.
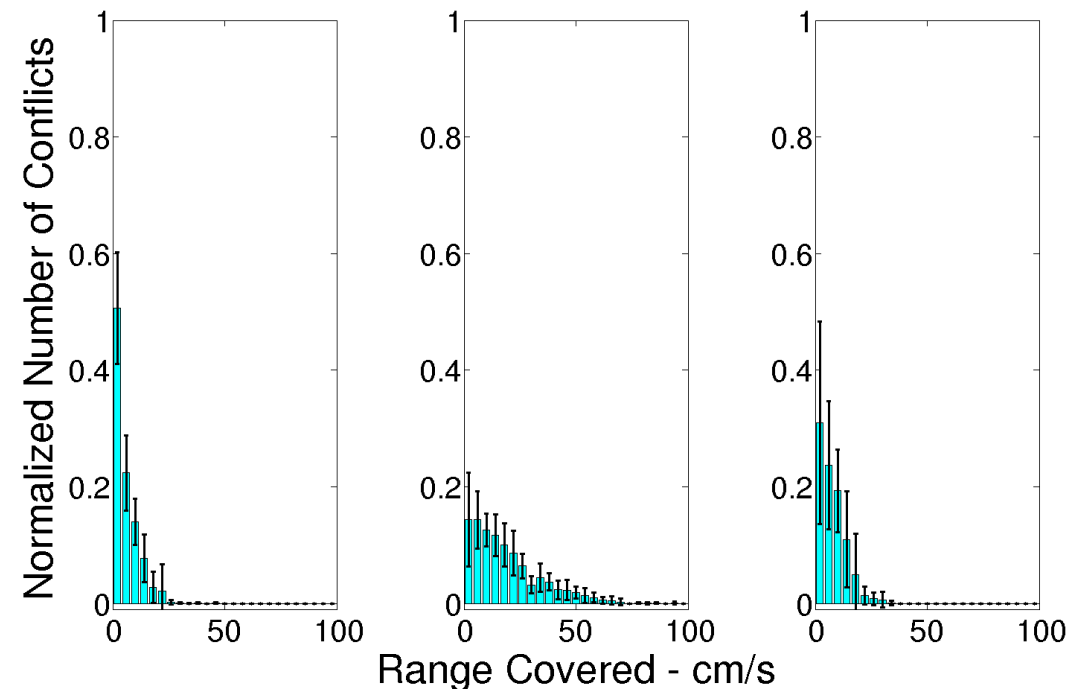

Figure 5.30: The $\Delta V$ distribution for seven influential neighbors and $\delta_{\text {attraction }}=10 \mathrm{~cm}$ for the 12-3 giant danio data set under the global coordinate system with the variable speed maneuver. 


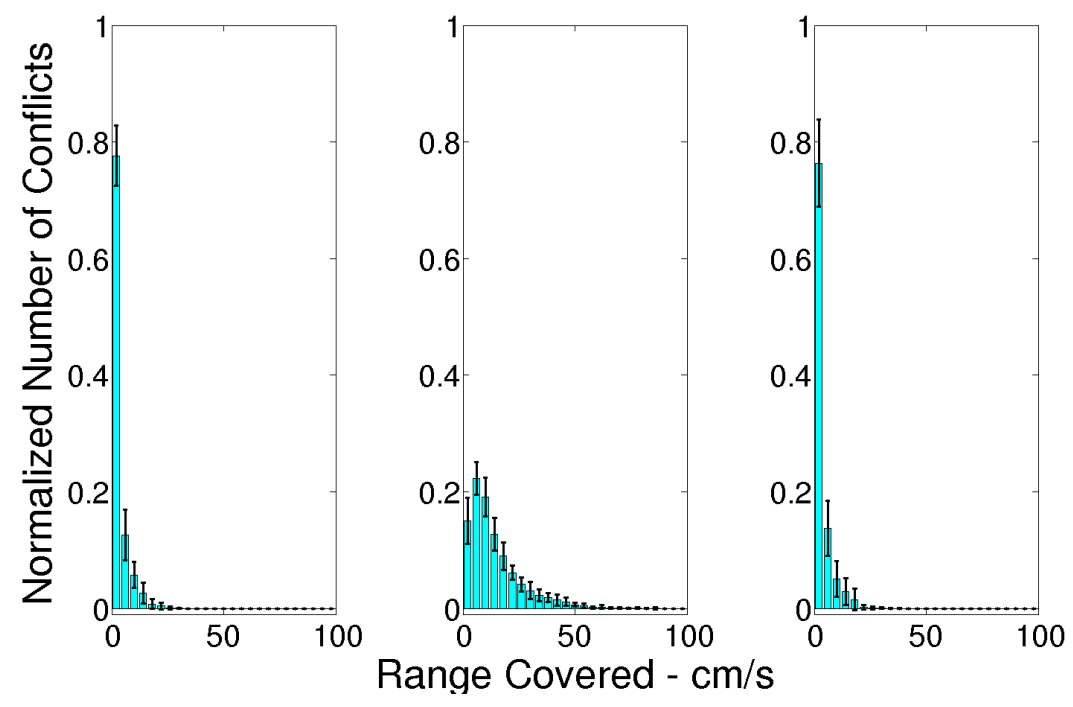

Figure 5.31: The $\Delta V$ distribution for three influential neighbors and $\delta_{\text {attraction }}=$ infinity for the 15-0 giant danio data set under the global coordinate system with the variable speed maneuver.
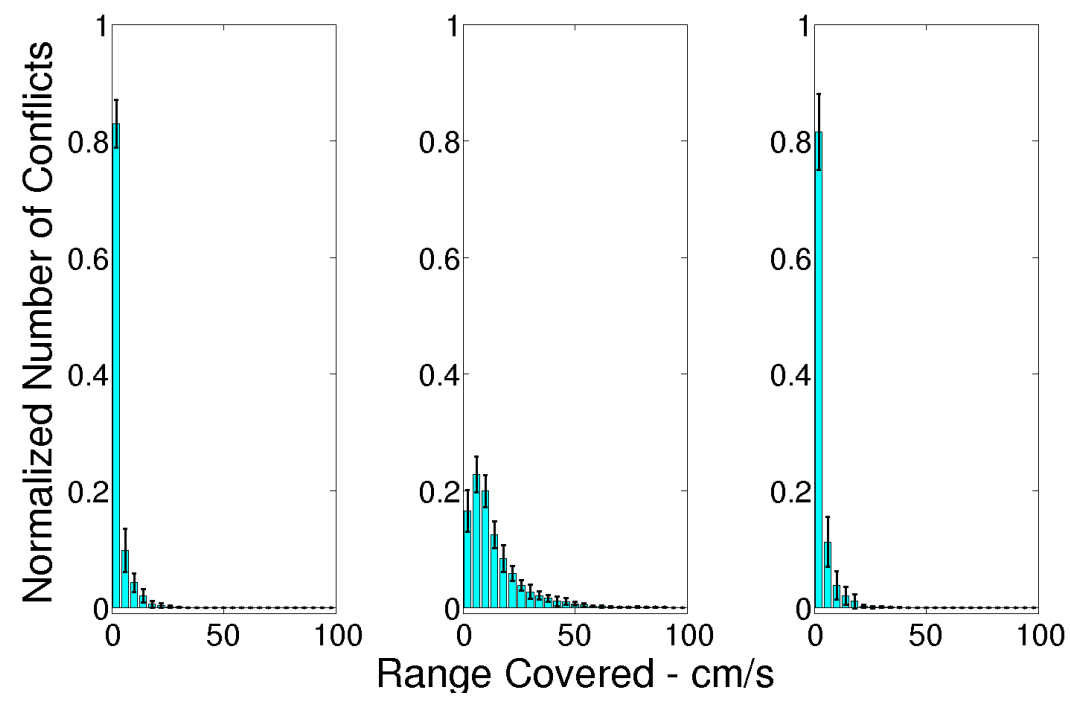

Figure 5.32: The $\Delta V$ distribution for seven influential neighbors and $\delta_{\text {attraction }}=$ infinity for the 15-0 giant danio data set under the global coordinate system with the variable speed maneuver. 


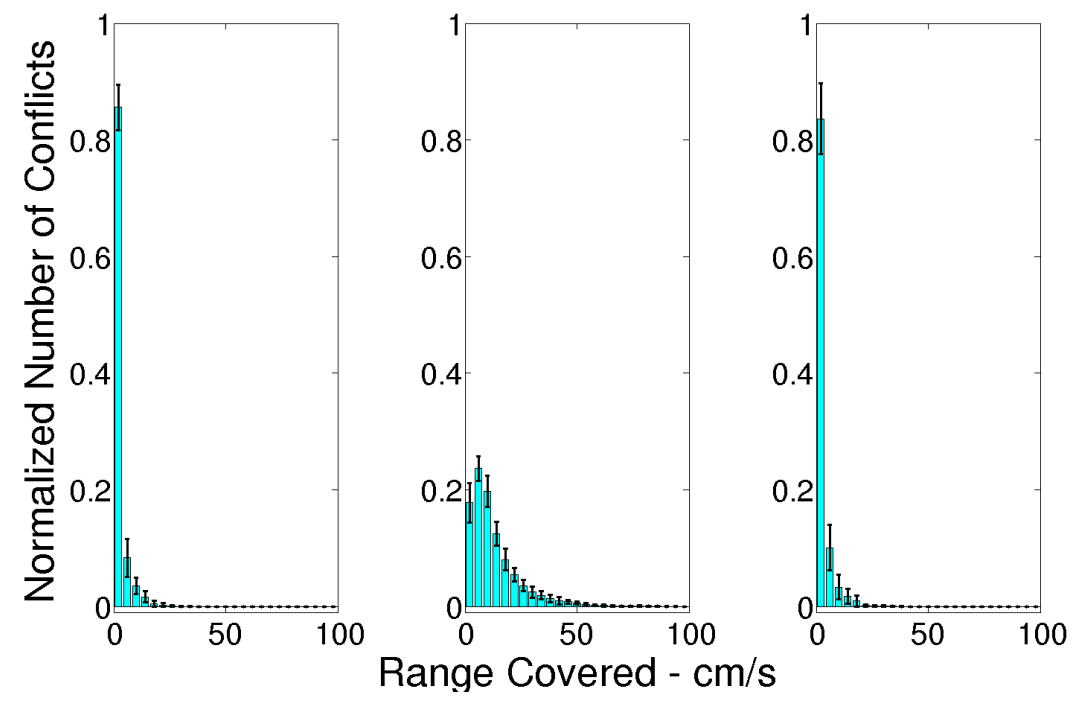

Figure 5.33: The $\Delta V$ distribution for the maximum number of influential neighbors and $\delta_{\text {attraction }}=$ infinity for the 15-0 giant danio data set under the global coordinate system with the variable speed maneuver..
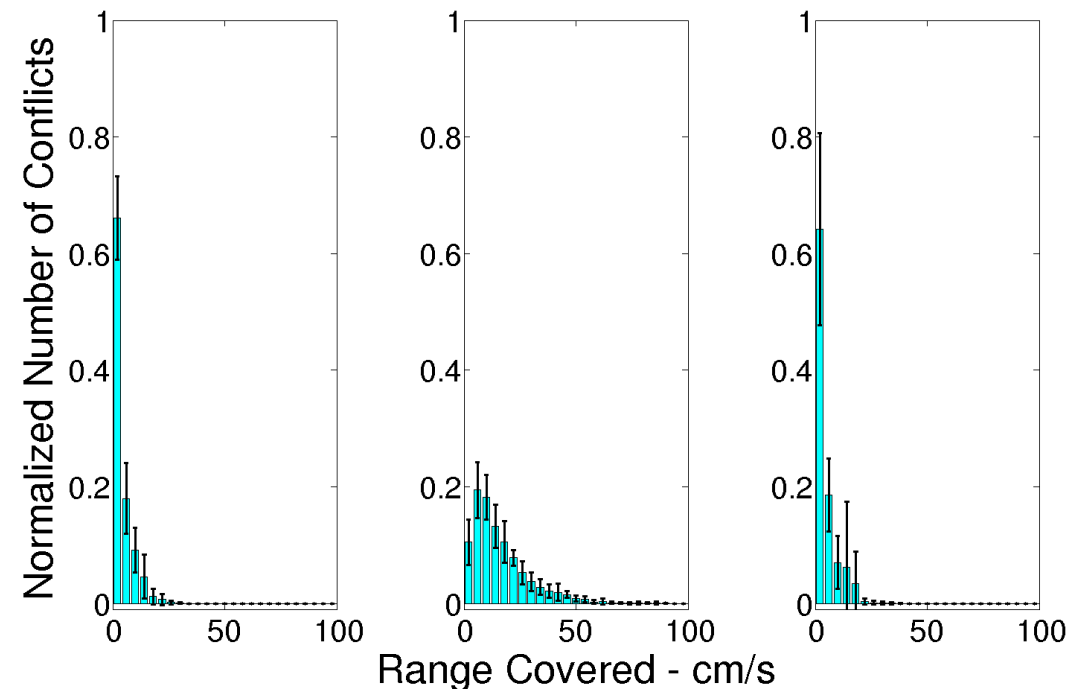

Figure 5.34: The $\Delta V$ distribution for seven influential neighbors and $\delta_{\text {attraction }}=20 \mathrm{~cm}$ for the 15-0 giant danio data set under the global coordinate system with the variable speed maneuver. 


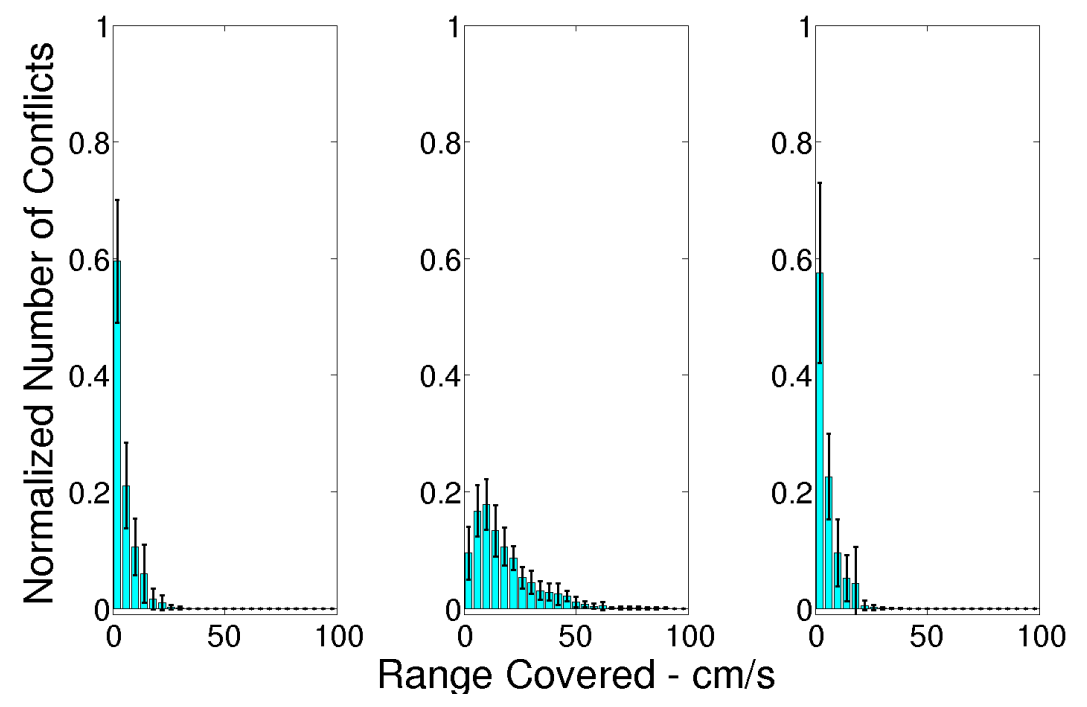

Figure 5.35: The $\Delta V$ distribution for seven influential neighbors and $\delta_{\text {attraction }}=15 \mathrm{~cm}$ for the 15-0 giant danio data set under the global coordinate system with the variable speed maneuver.
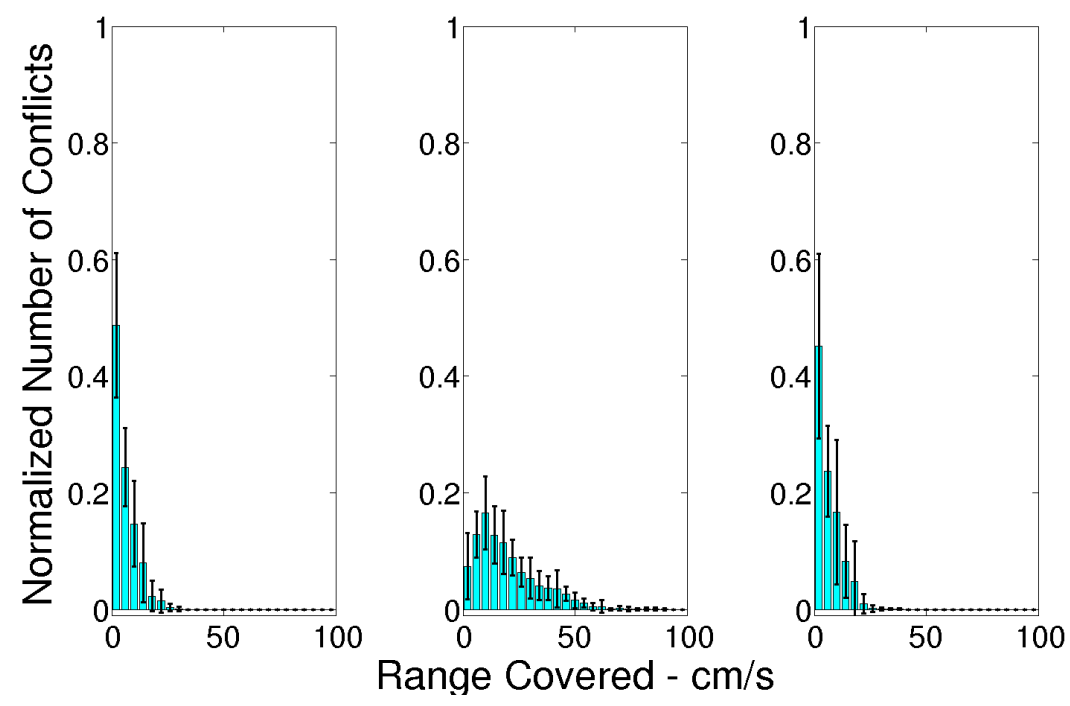

Figure 5.36: The $\Delta V$ distribution for seven influential neighbors and $\delta_{\text {attraction }}=10 \mathrm{~cm}$ for the 15-0 giant danio data set under the global coordinate system with the variable speed maneuver. 

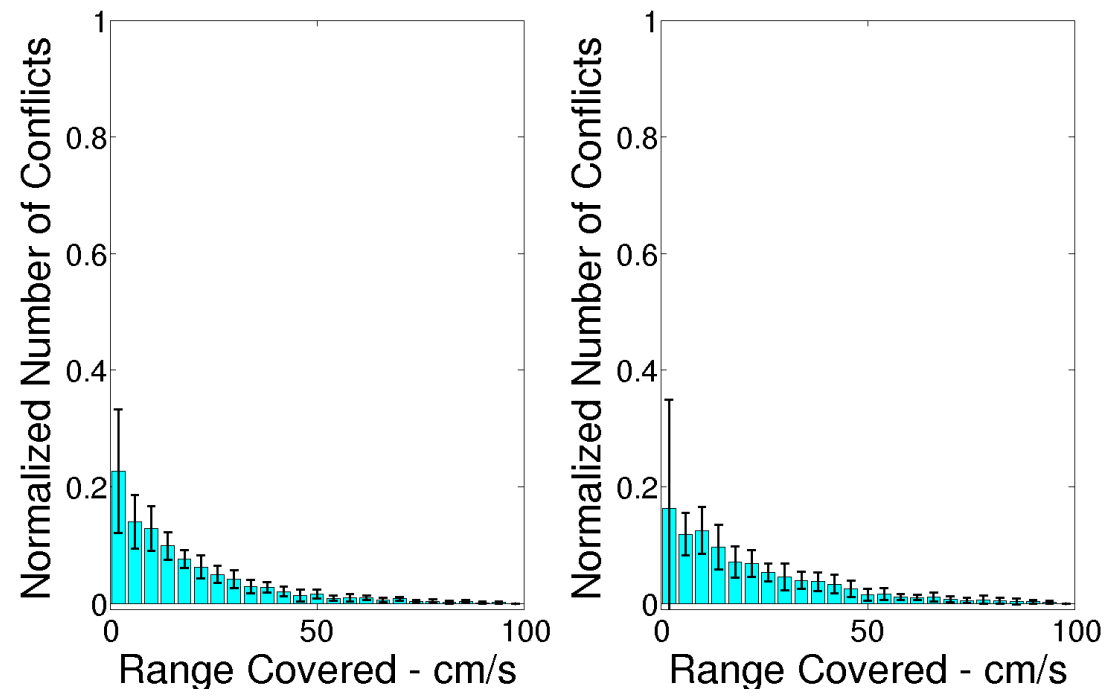

Figure 5.37: The $\Delta V$ distribution for three influential neighbors and $\delta_{\text {attraction }}=$ infinity for the 0-15 giant danio data set under the body-fixed coordinate system with the constant speed maneuver. 

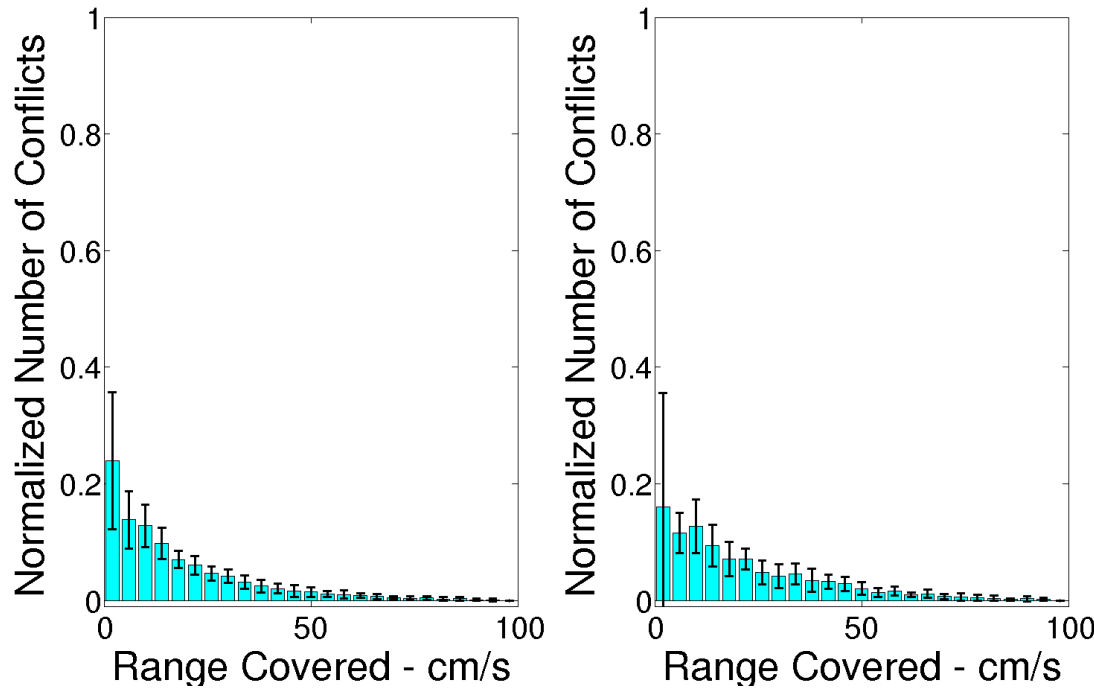

Figure 5.38: The $\Delta V$ distribution for seven influential neighbors and $\delta_{\text {attraction }}=$ infinity for the 0-15 giant danio data set under the body-fixed coordinate system with the constant speed maneuver.
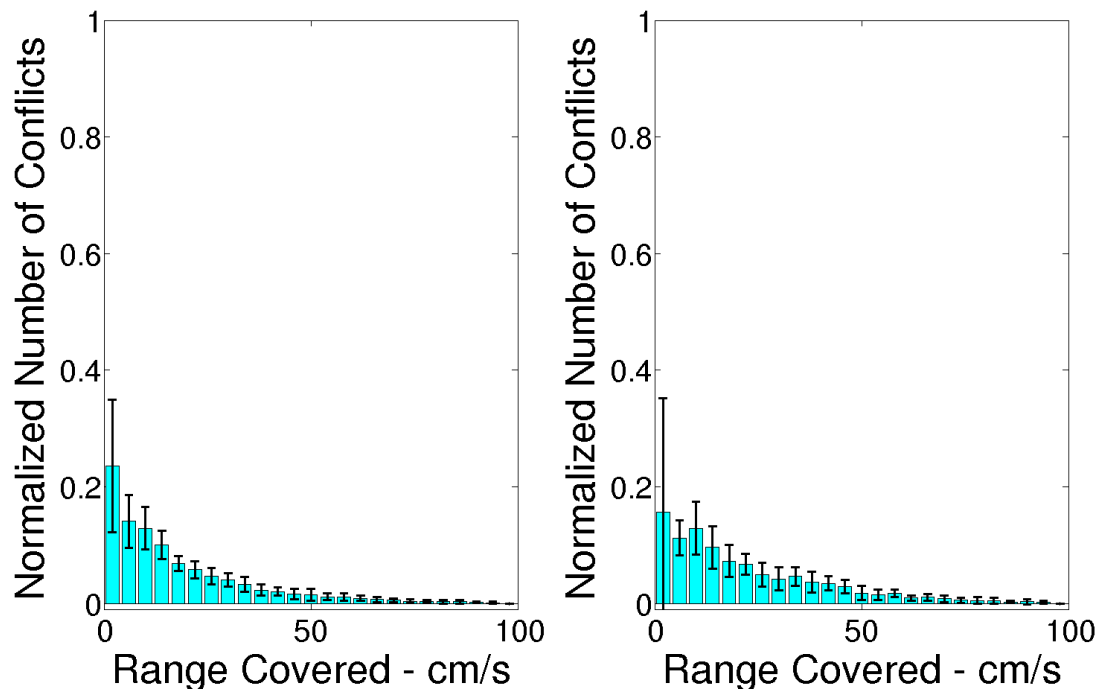

Figure 5.39: The $\Delta V$ distribution for maximum number of influential neighbors and $\delta_{\text {attraction }}=$ infinity for the 0-15 giant danio data set under the body-fixed coordinate system with the constant speed maneuver. 

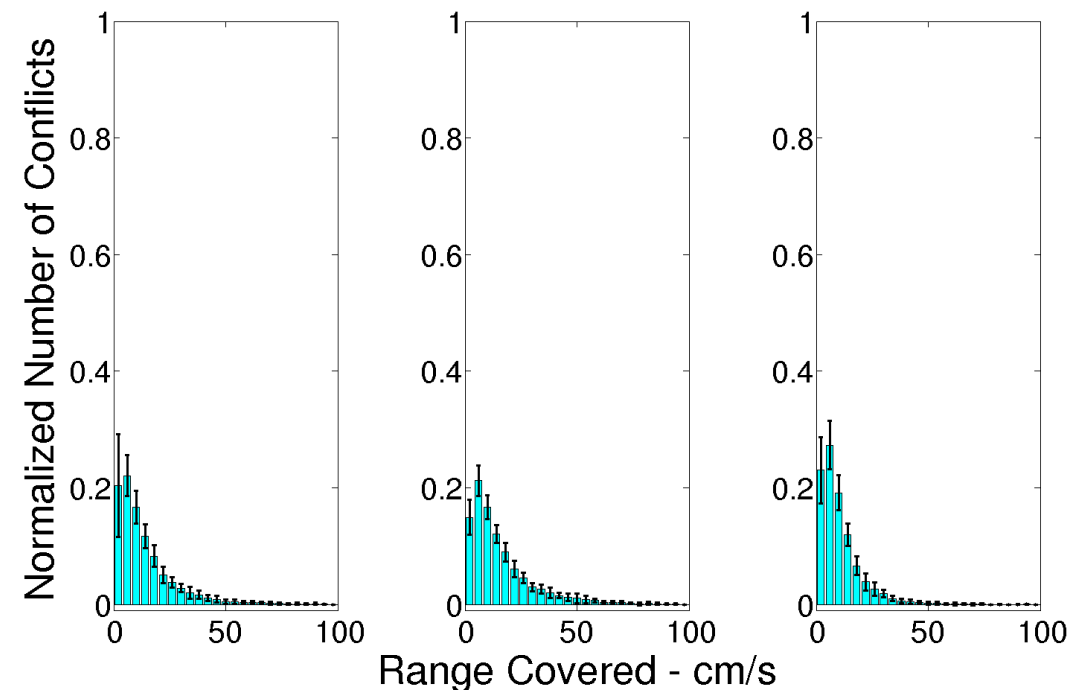

Figure 5.40: The $\Delta V$ distribution for three influential neighbors and $\delta_{\text {attraction }}=$ infinity for the 6-9 giant danio data set under the body-fixed coordinate system with the constant speed maneuver.
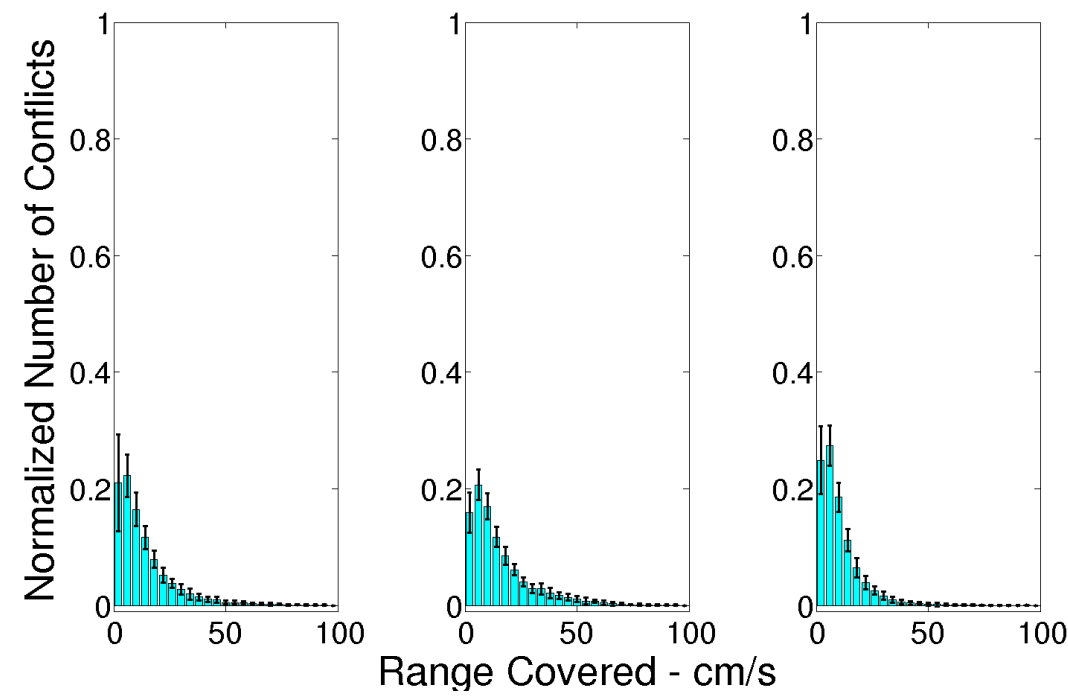

Figure 5.41: The $\Delta V$ distribution for seven influential neighbors and $\delta_{\text {attraction }}=$ infinity for the 6-9 giant danio data set under the body-fixed coordinate system with the constant speed maneuver. 

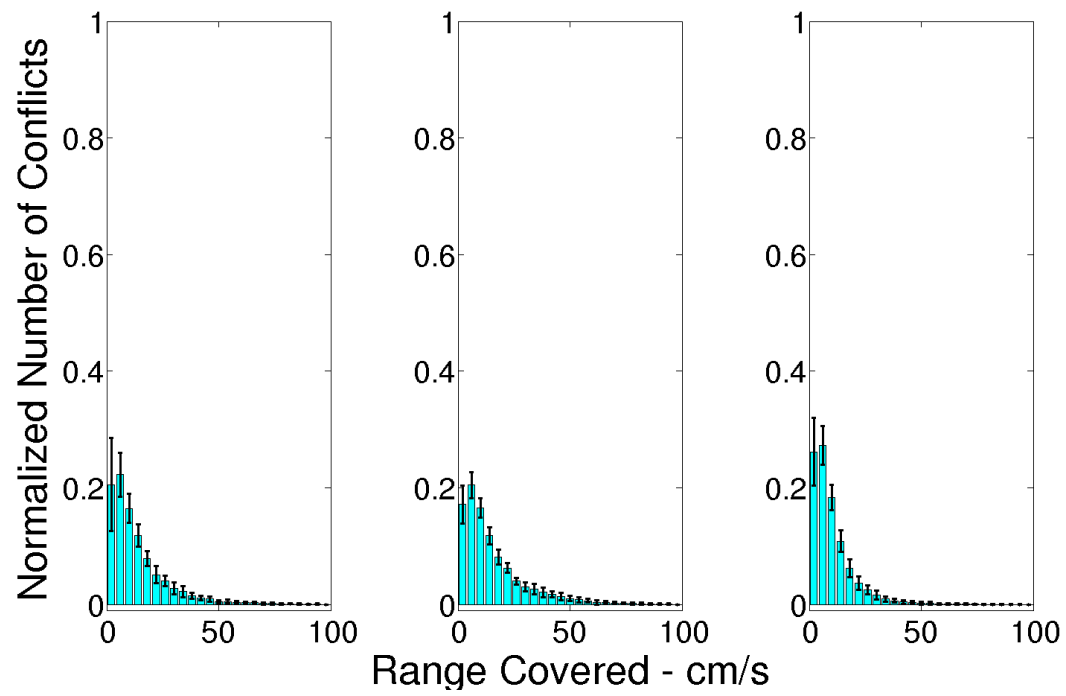

Figure 5.42: The $\Delta V$ distribution for the maximum number of influential neighbors and $\delta_{\text {attraction }}=$ infinity for the 6-9 giant danio data set under the body-fixed coordinate system with the constant speed maneuver.
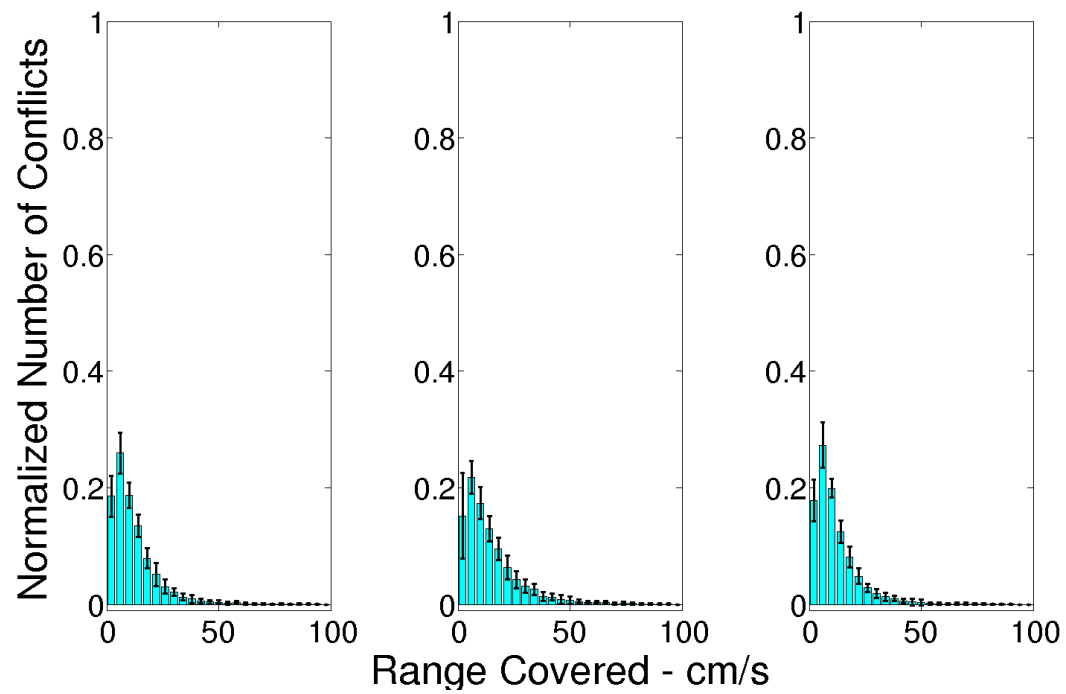

Figure 5.43: The $\Delta V$ distribution for three influential neighbors and $\delta_{\text {attraction }}=$ infinity for the 9-6 giant danio data set under the body-fixed coordinate system with the constant speed maneuver. 

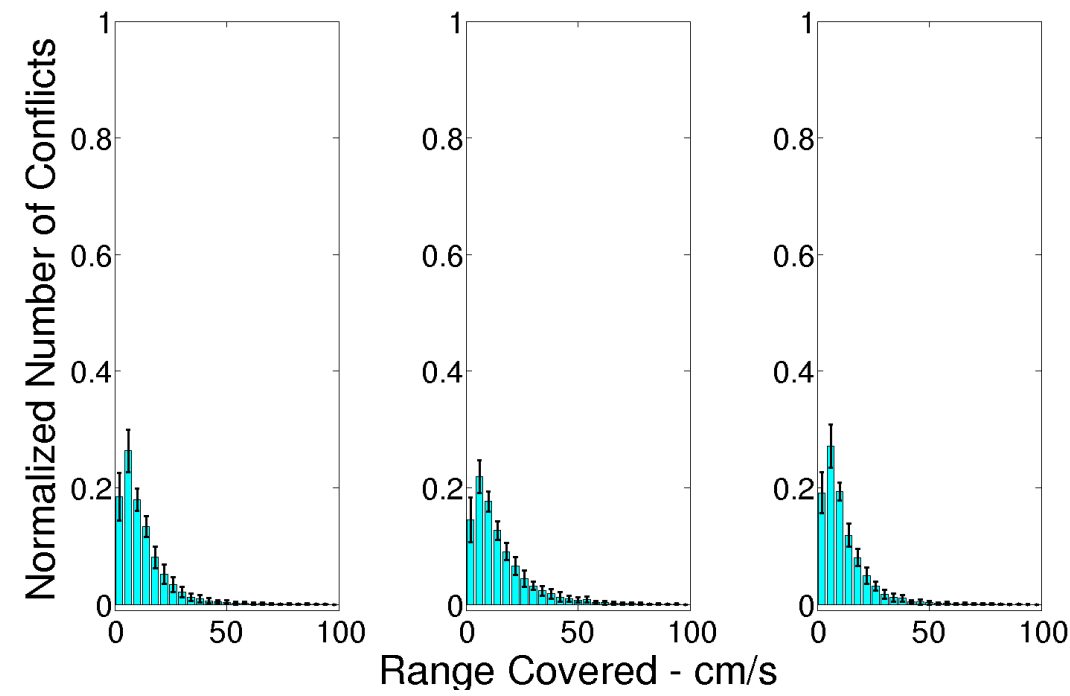

Figure 5.44: The $\Delta V$ distribution for seven influential neighbors and $\delta_{\text {attraction }}=$ infinity for the 9-6 giant danio data set under the body-fixed coordinate system with the constant speed maneuver.
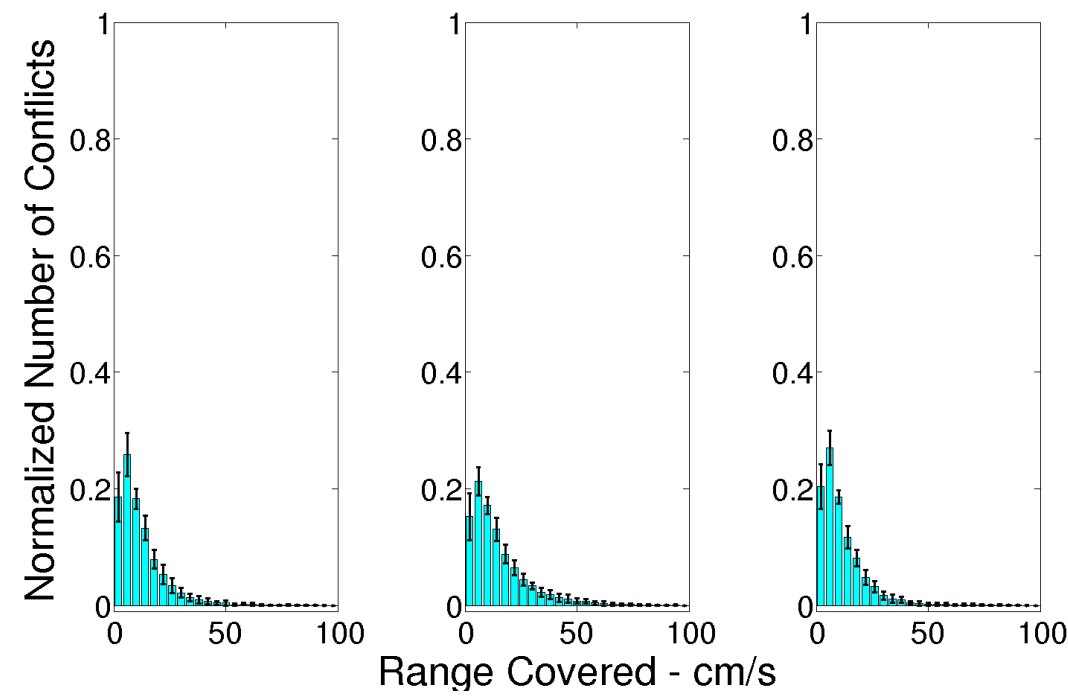

Figure 5.45: The $\Delta V$ distribution for the maximum number of influential neighbors and $\delta_{\text {attraction }}=$ infinity for the 9-6 giant danio data set under the body-fixed coordinate system with the constant speed maneuver. 

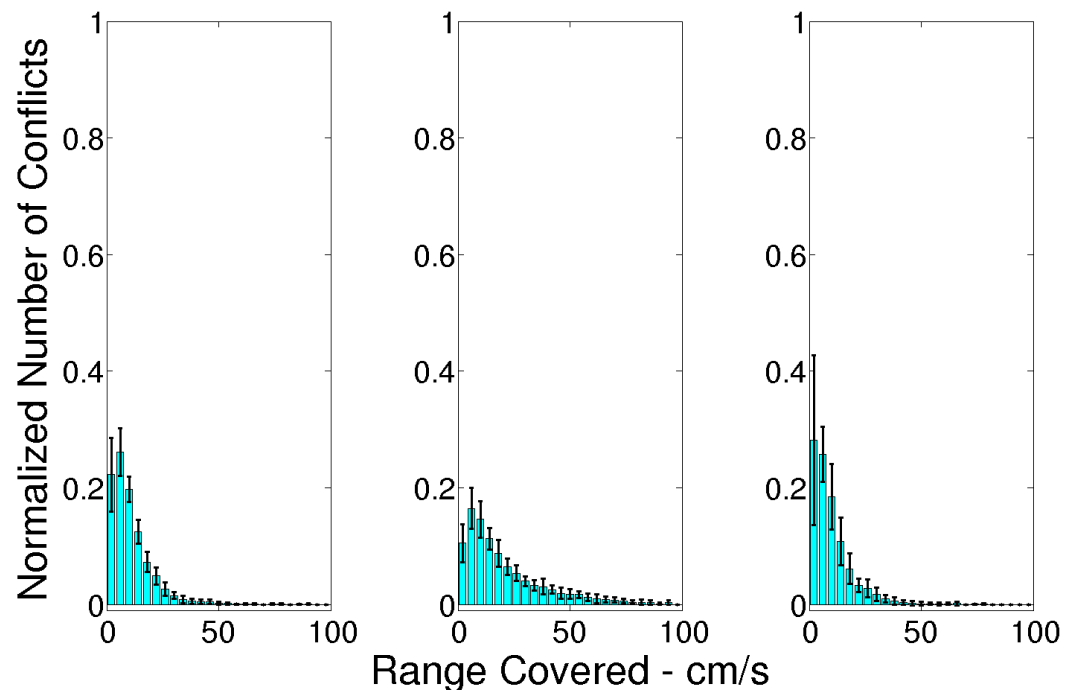

Figure 5.46: The $\Delta V$ distribution for three influential neighbors and $\delta_{\text {attraction }}=$ infinity for the 12-3 giant danio data set under the body-fixed coordinate system with the constant speed maneuver.
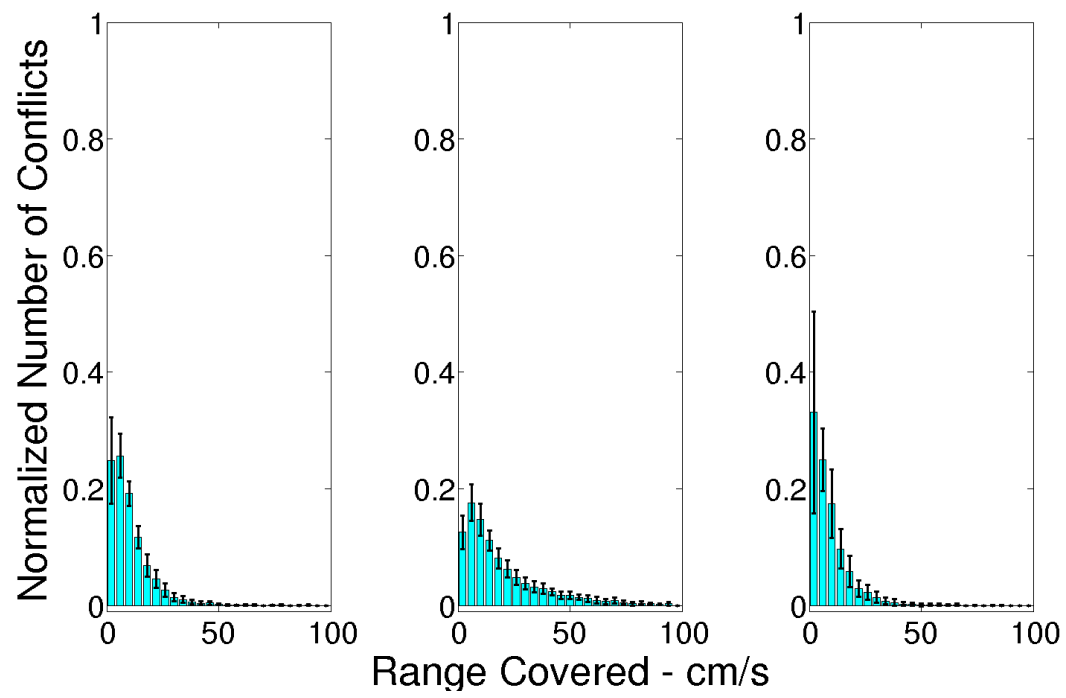

Figure 5.47: The $\Delta V$ distribution for seven influential neighbors and $\delta_{\text {attraction }}=$ infinity for the 12-3 giant danio data set under the body-fixed coordinate system with the constant speed maneuver. 

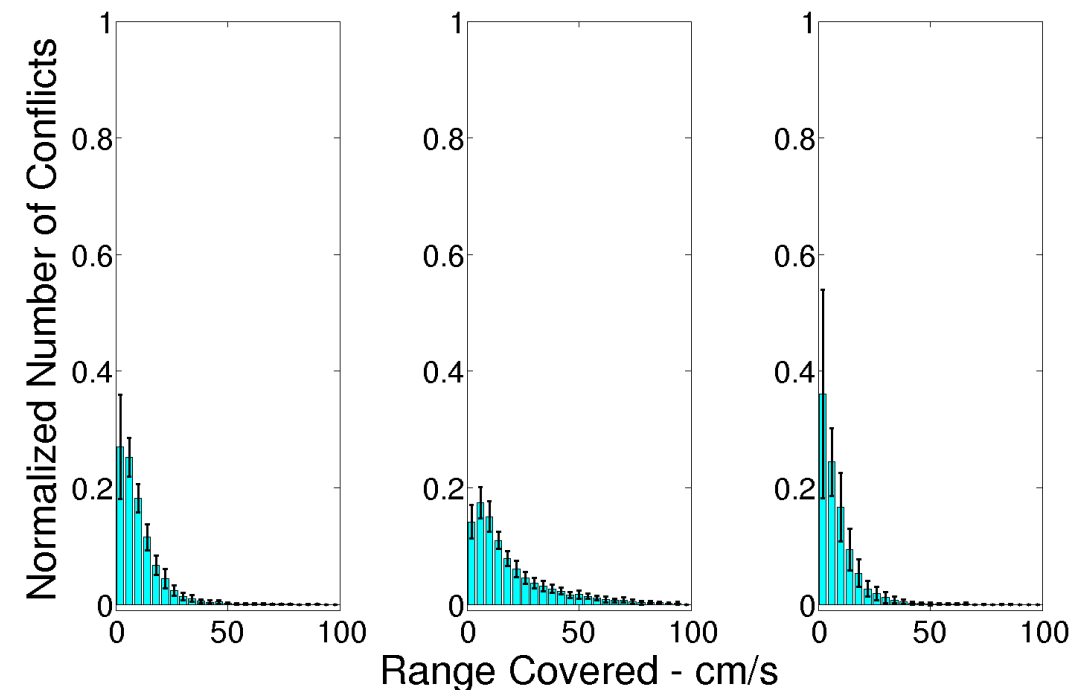

Figure 5.48: The $\Delta V$ distribution for the maximum number of influential neighbors and $\delta_{\text {attraction }}=$ infinity for the 12-3 giant danio data set under the body-fixed coordinate system with the constant speed maneuver.
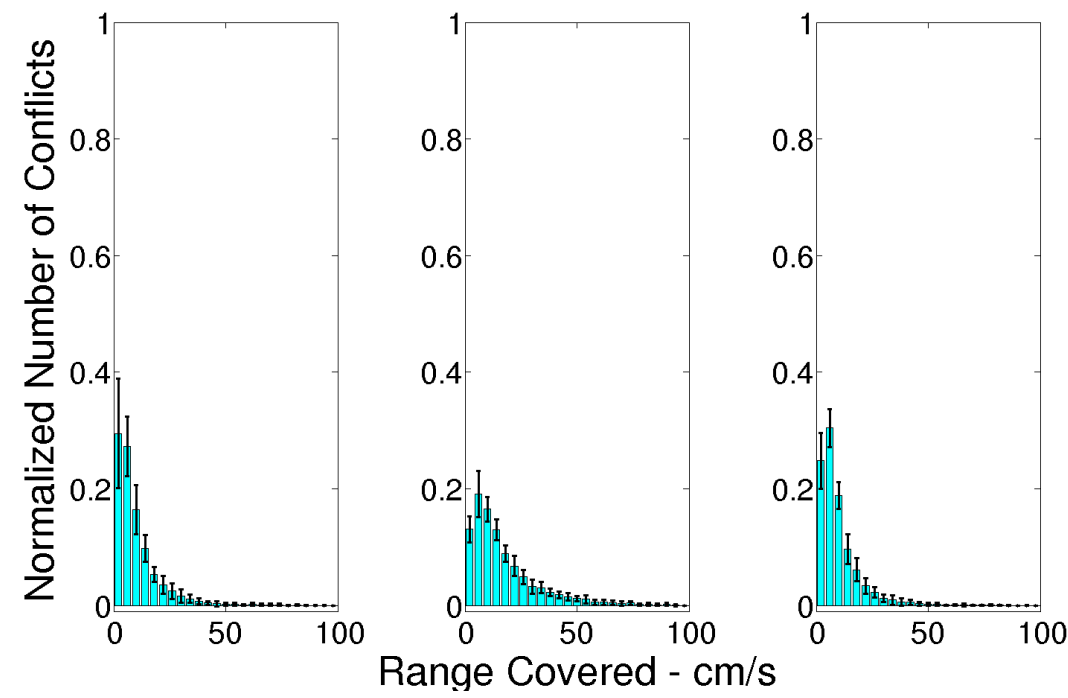

Figure 5.49: The $\Delta V$ distribution for three influential neighbors and $\delta_{\text {attraction }}=$ infinity for the 15-0 giant danio data set under the body-fixed coordinate system with the constant speed maneuver. 

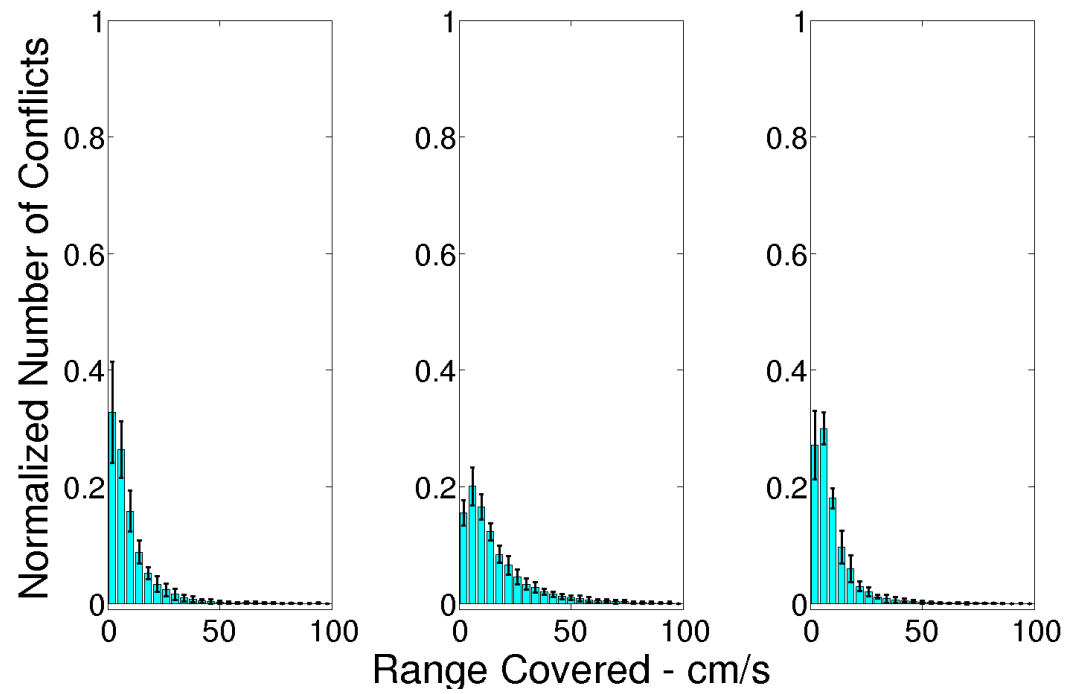

Figure 5.50: The $\Delta V$ distribution for seven influential neighbors and $\delta_{\text {attraction }}=$ infinity for the 15-0 giant danio data set under the body-fixed coordinate system with the constant speed maneuver.
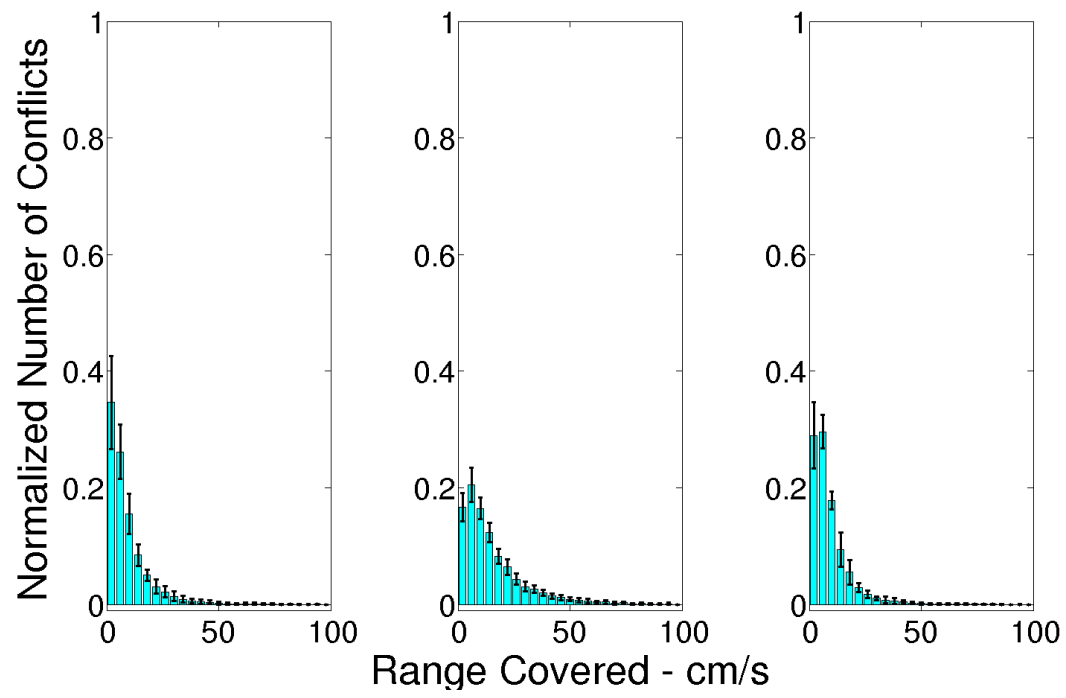

Figure 5.51: The $\Delta V$ distribution for the maximum number of influential neighbors and $\delta_{\text {attraction }}=$ infinity for the 15-0 giant danio data set under the body-fixed coordinate system with the constant speed maneuver. 

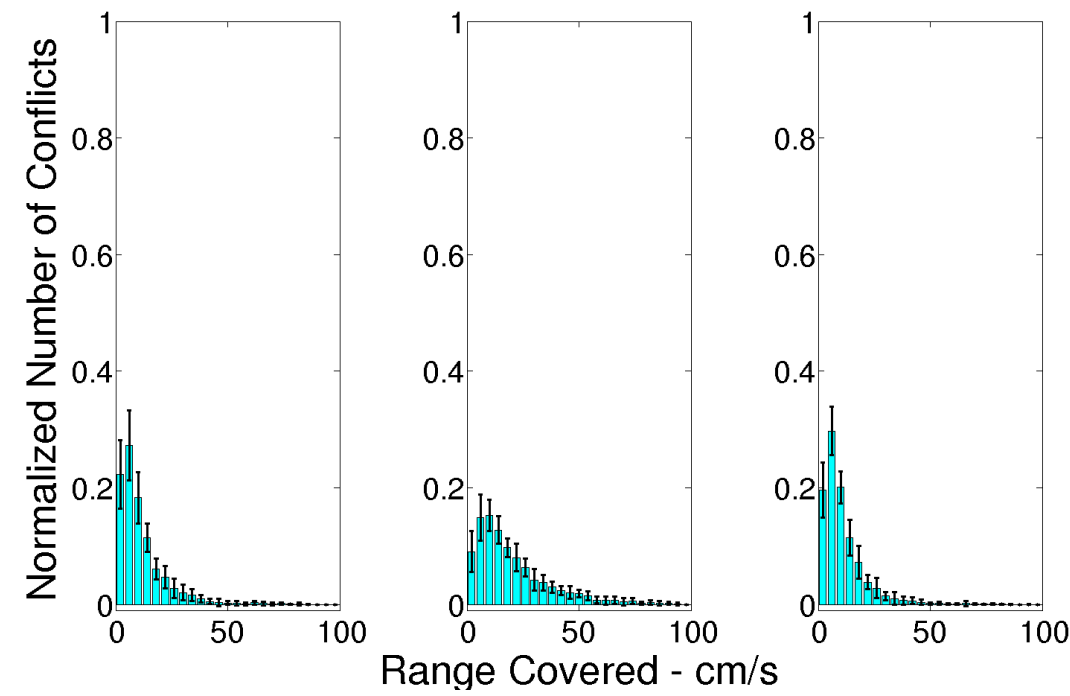

Figure 5.52: The $\Delta V$ distribution for the maximum number of influential neighbors and $\delta_{\text {attraction }}=20 \mathrm{~cm}$ for the $15-0$ giant danio data set under the body-fixed coordinate system with the constant speed maneuver.
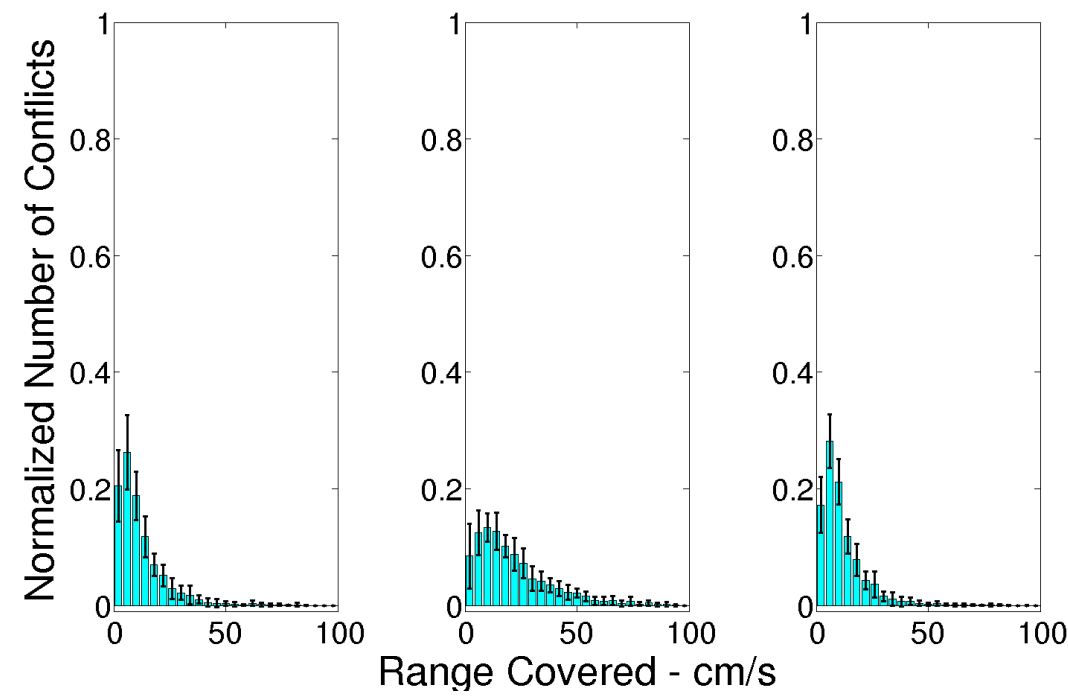

Figure 5.53: The $\Delta V$ distribution for the maximum number of influential neighbors and $\delta_{\text {attraction }}=15 \mathrm{~cm}$ for the 15 -0 giant danio data set under the body-fixed coordinate system with the constant speed maneuver. 

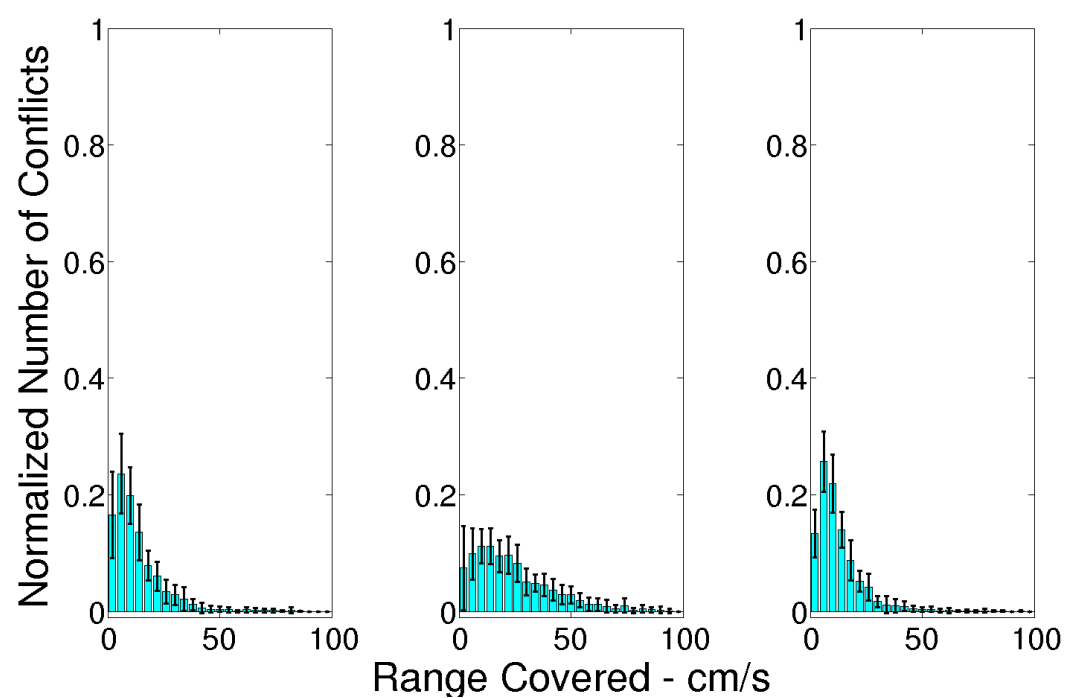

Figure 5.54: The $\Delta V$ distribution for the maximum number of influential neighbors and $\delta_{\text {attraction }}=10 \mathrm{~cm}$ for the 15 -0 giant danio data set under the body-fixed coordinate system with the constant speed maneuver. 

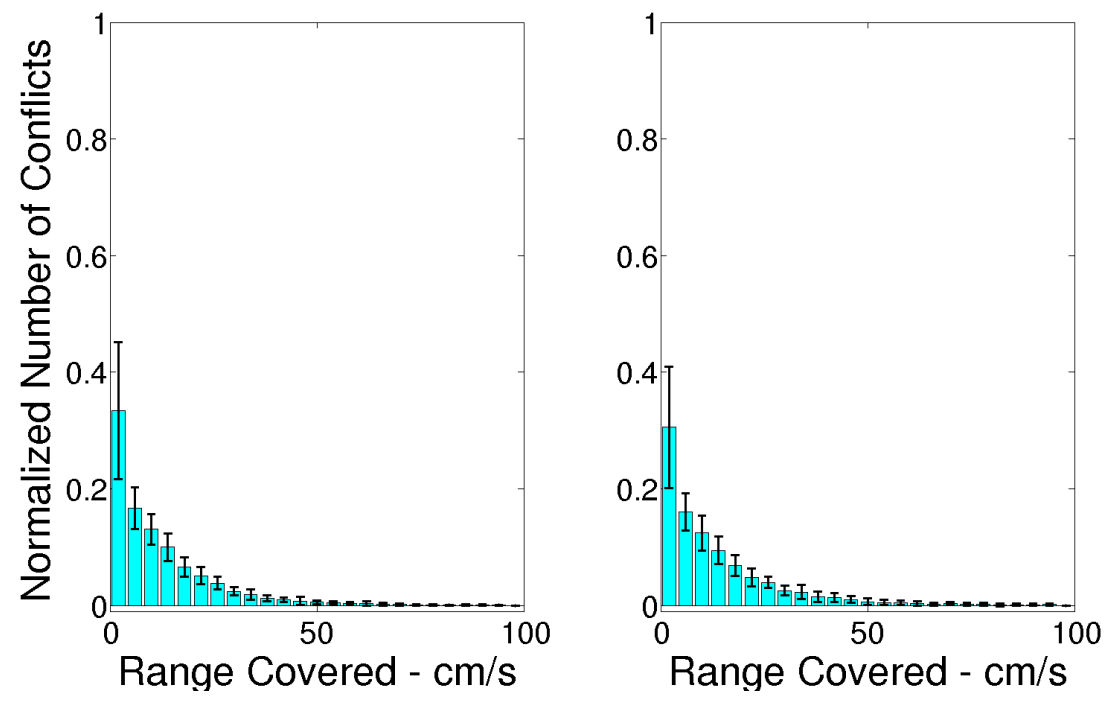

Figure 5.55: The $\Delta V$ distribution for three influential neighbors and $\delta_{\text {attraction }}=$ infinity for the 0-15 giant danio data set under the body-fixed coordinate system with the variable speed maneuver. 

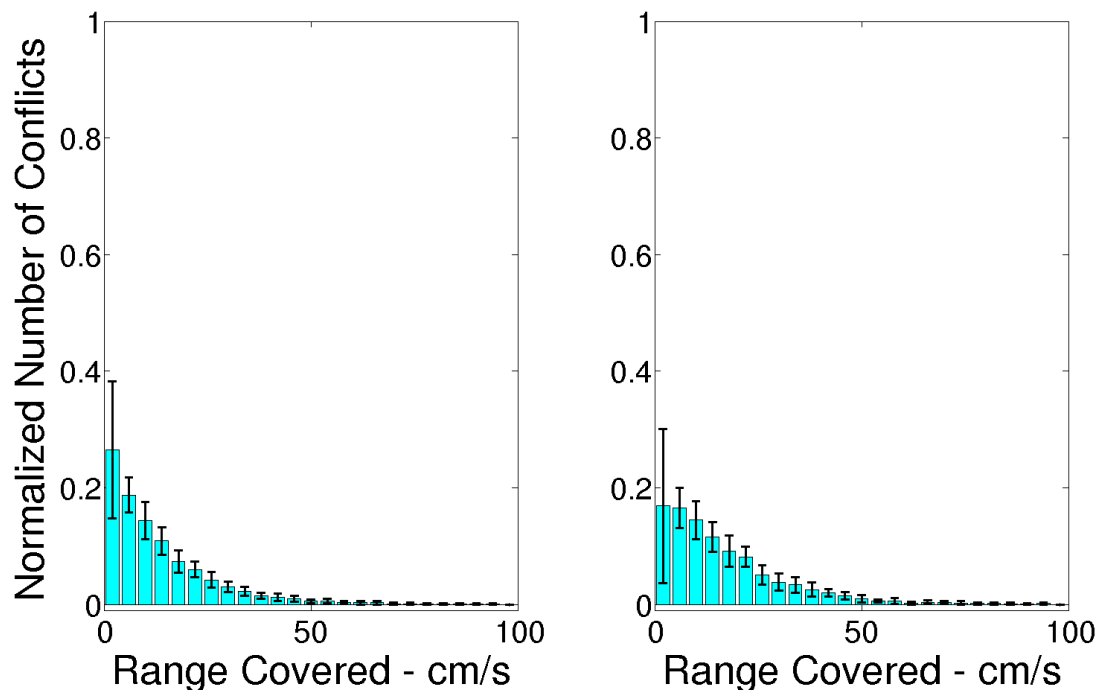

Figure 5.56: The $\Delta V$ distribution for seven influential neighbors and $\delta_{\text {attraction }}=$ infinity for the 0-15 giant danio data set under the body-fixed coordinate system with the variable speed maneuver.
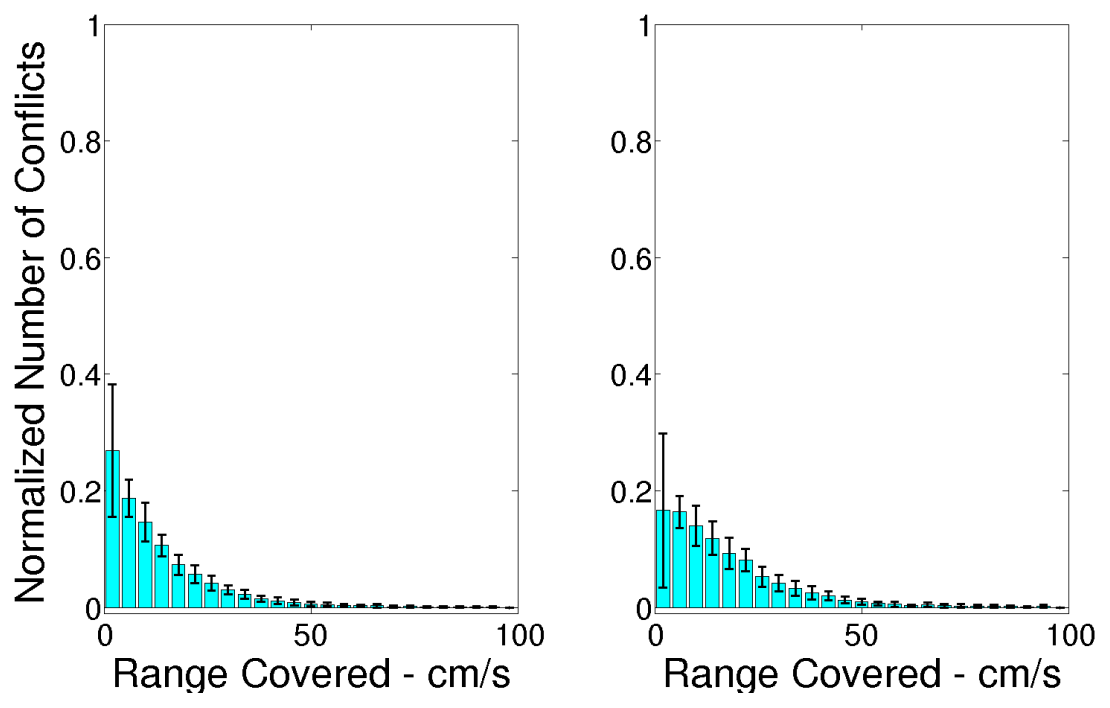

Figure 5.57: The $\Delta V$ distribution for the maximum number of influential neighbors and $\delta_{\text {attraction }}=$ infinity for the 0-15 giant danio data set under the body-fixed coordinate system with the variable speed maneuver. 

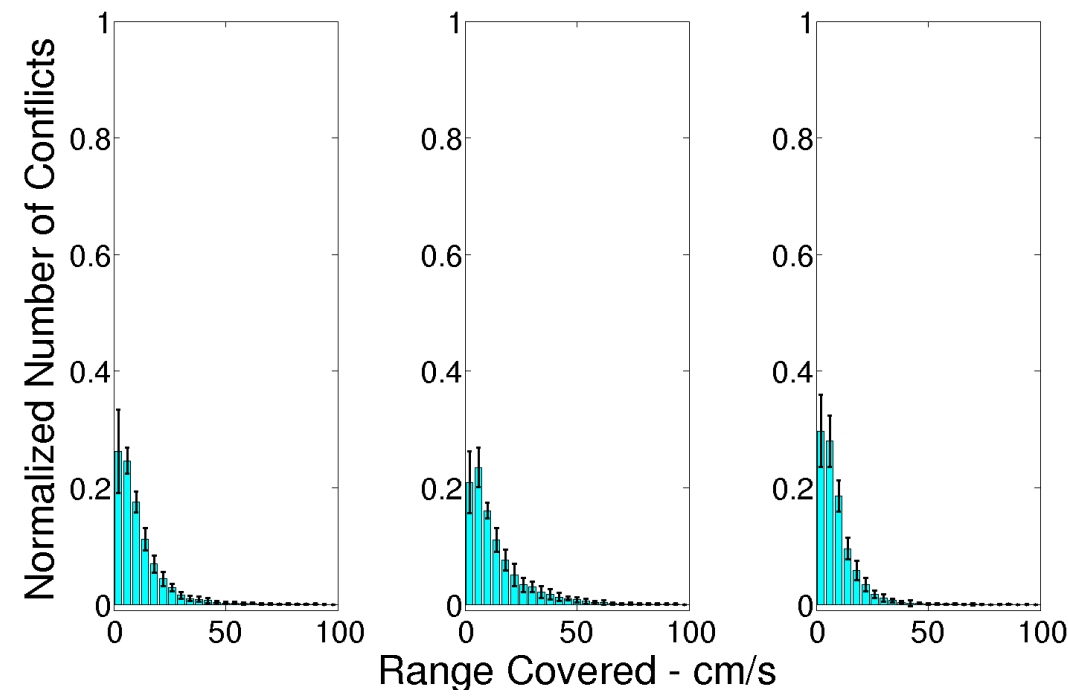

Figure 5.58: The $\Delta V$ distribution for three influential neighbors and $\delta_{\text {attraction }}=$ infinity for the 6-9 giant danio data set under the body-fixed coordinate system with the variable speed maneuver.
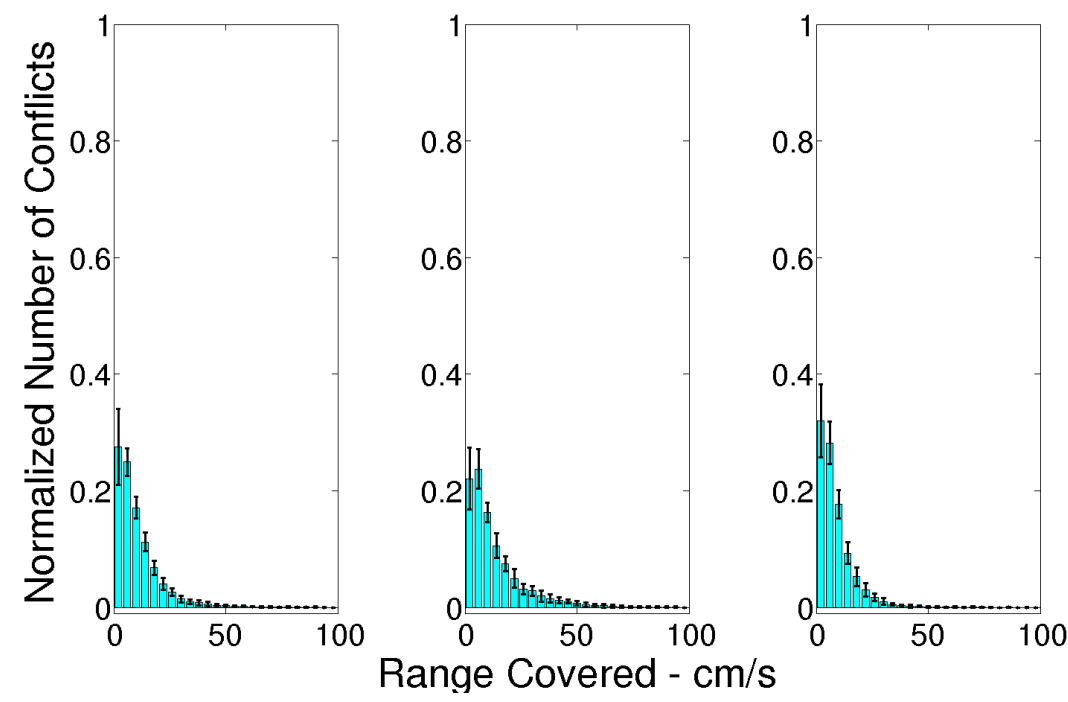

Figure 5.59: The $\Delta V$ distribution for seven influential neighbors and $\delta_{\text {attraction }}=$ infinity for the 6-9 giant danio data set under the body-fixed coordinate system with the variable speed maneuver. 

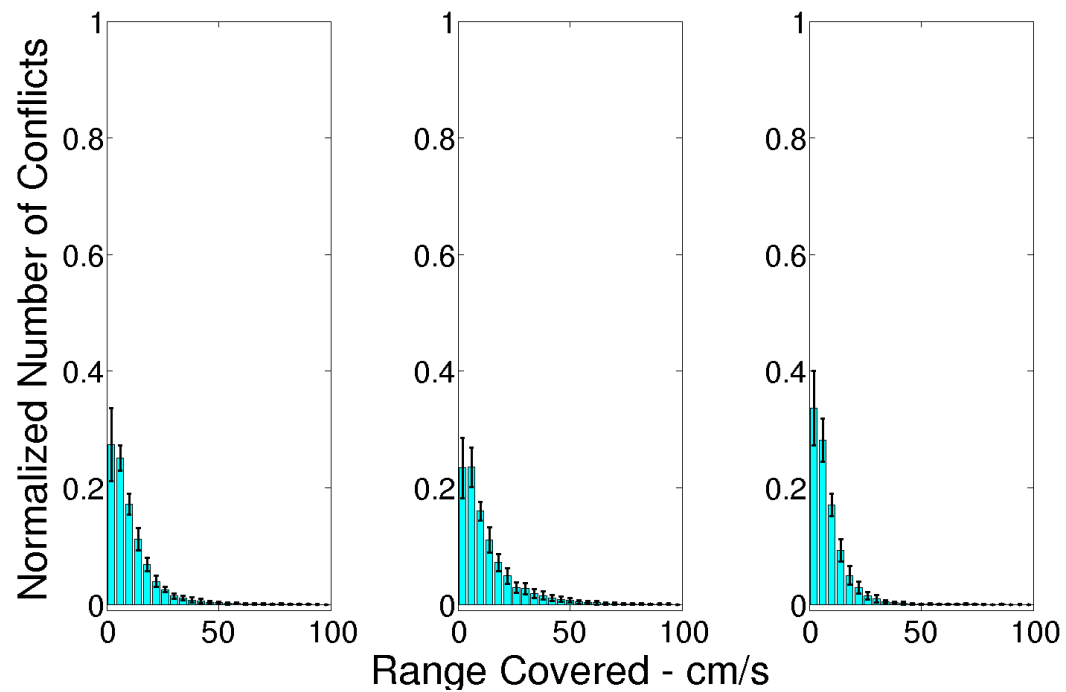

Figure 5.60: The $\Delta V$ distribution for the maximum number of influential neighbors and $\delta_{\text {attraction }}=$ infinity for the 6-9 giant danio data set under the body-fixed coordinate system with the variable speed maneuver.
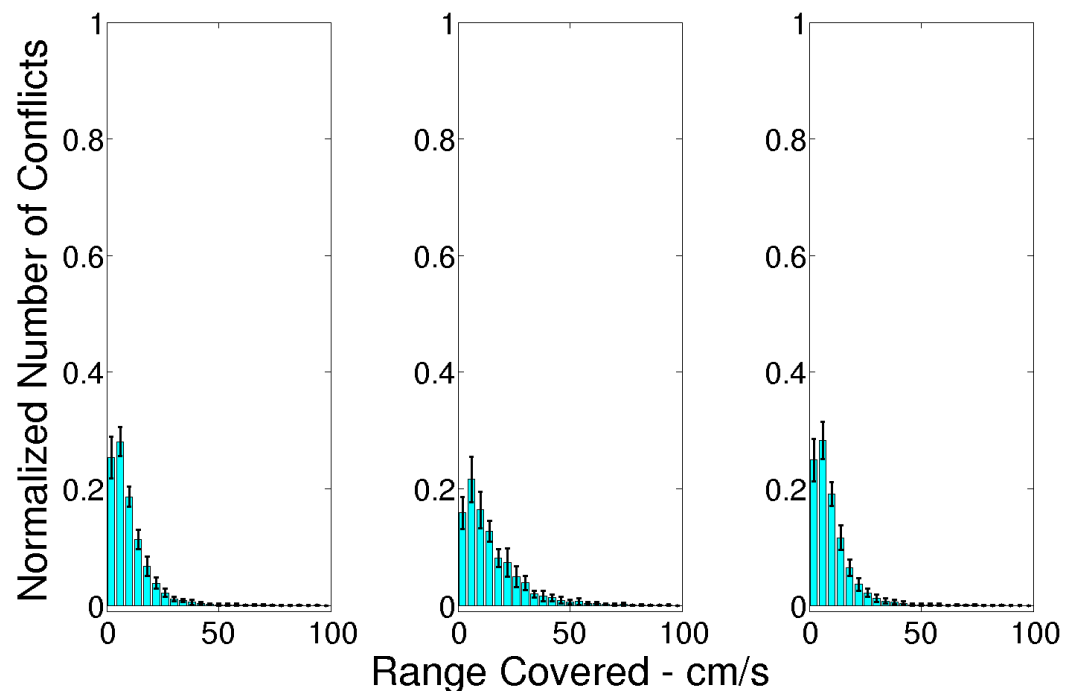

Figure 5.61: The $\Delta V$ distribution for three influential neighbors and $\delta_{\text {attraction }}=$ infinity for the 9-6 giant danio data set under the body-fixed coordinate system with the variable speed maneuver. 


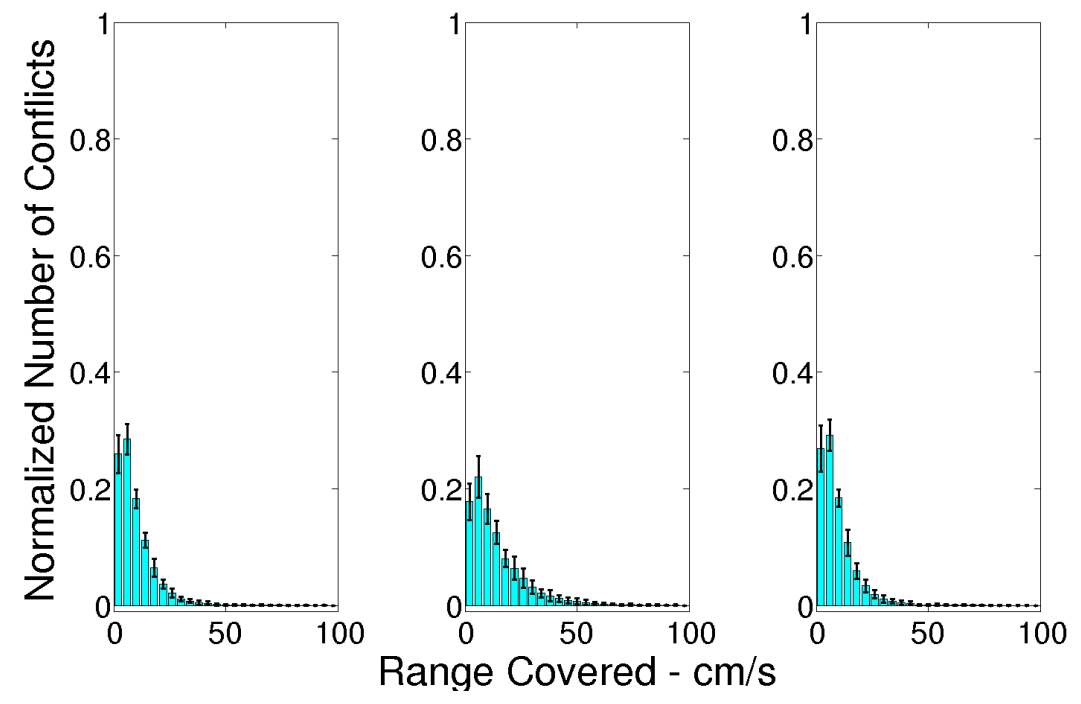

Figure 5.62: The $\Delta V$ distribution for seven influential neighbors and $\delta_{\text {attraction }}=$ infinity for the 9-6 giant danio data set under the body-fixed coordinate system with the variable speed maneuver.
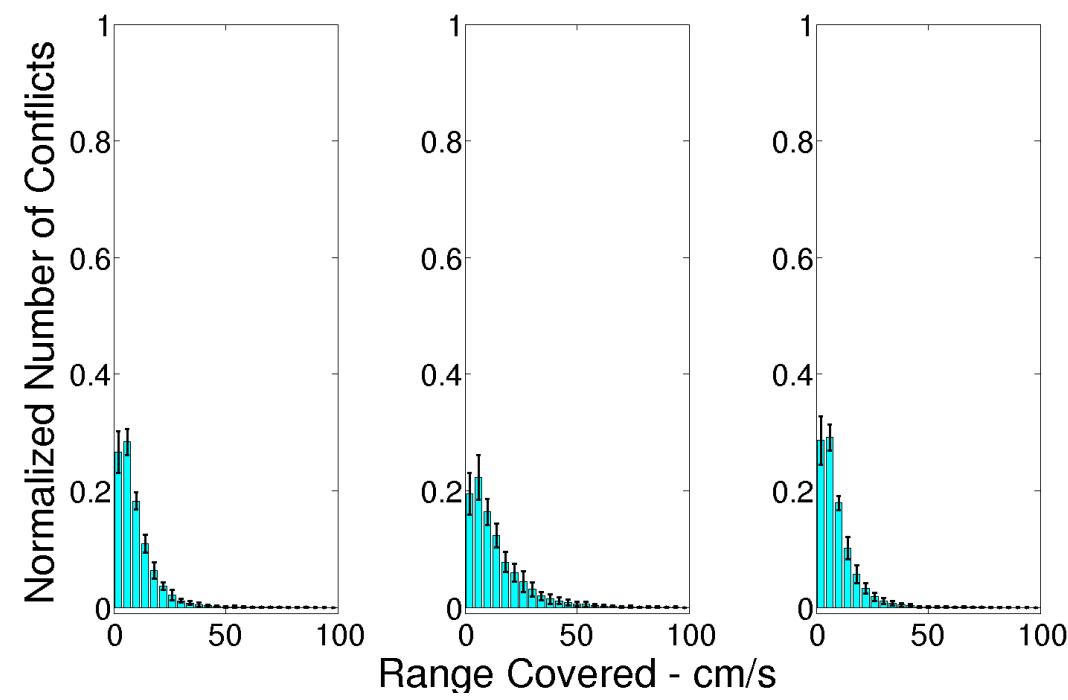

Figure 5.63: The $\Delta V$ distribution for the maximum number of influential neighbors and $\delta_{\text {attraction }}=$ infinity for the 9-6 giant danio data set under the body-fixed coordinate system with the variable speed maneuver. 

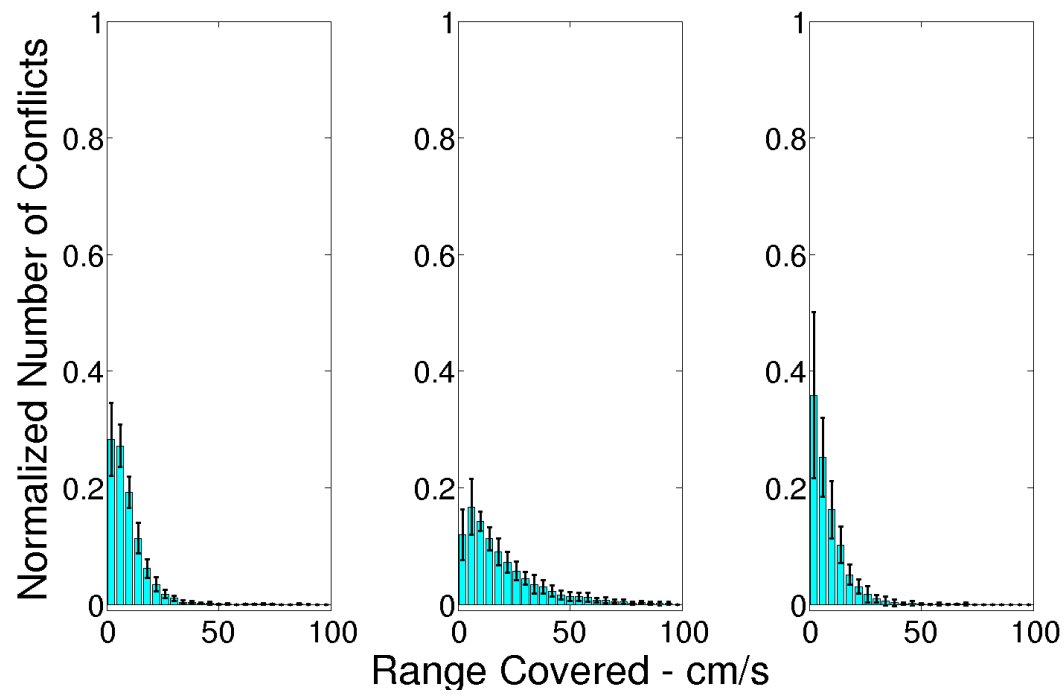

Figure 5.64: The $\Delta V$ distribution for three influential neighbors and $\delta_{\text {attraction }}=$ infinity for the 12-3 giant danio data set under the body-fixed coordinate system with the variable speed maneuver.
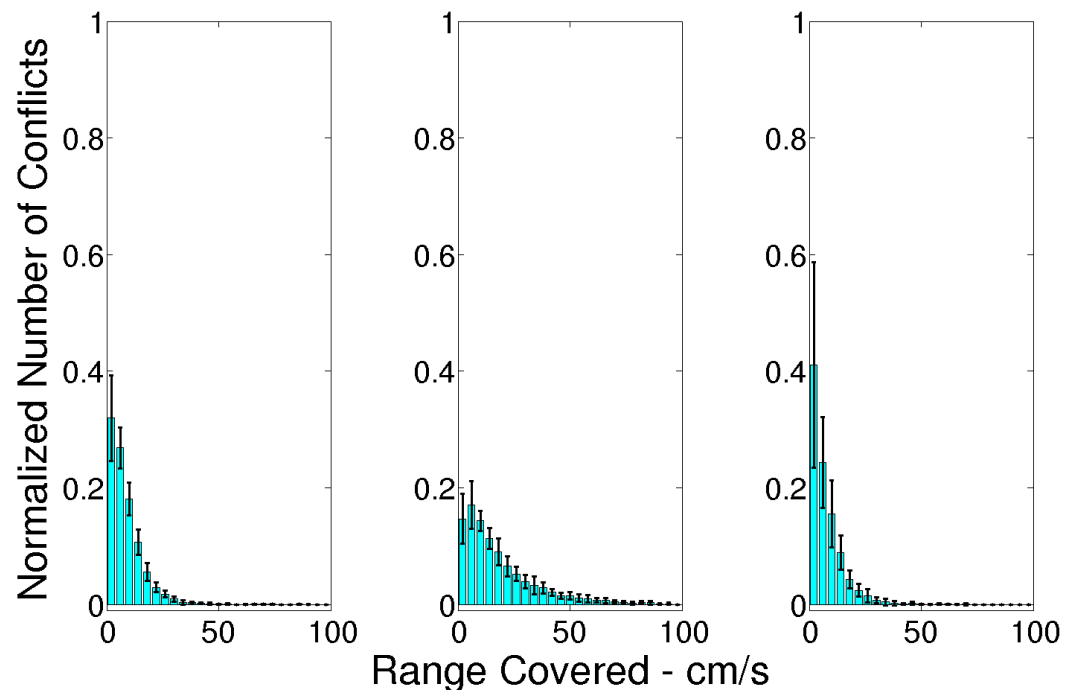

Figure 5.65: The $\Delta V$ distribution for seven influential neighbors and $\delta_{\text {attraction }}=$ infinity for the 12-3 giant danio data set under the body-fixed coordinate system with the variable speed maneuver. 

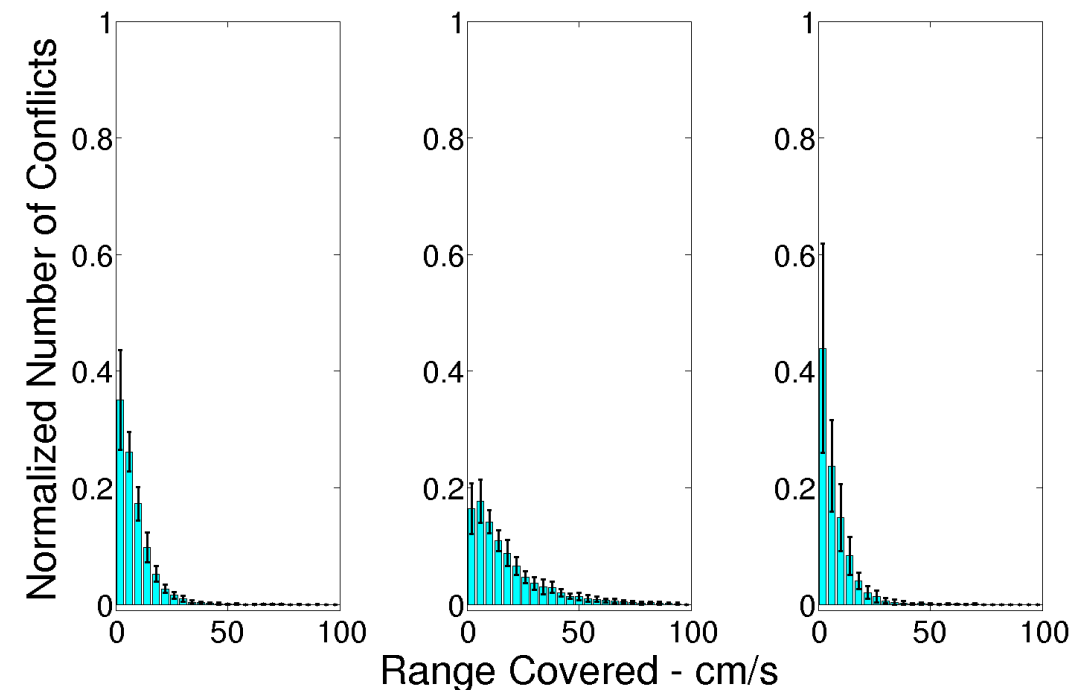

Figure 5.66: The $\Delta V$ distribution for the maximum number of influential neighbors and $\delta_{\text {attraction }}=$ infinity for the 12-3 giant danio data set under the body-fixed coordinate system with the variable speed maneuver.
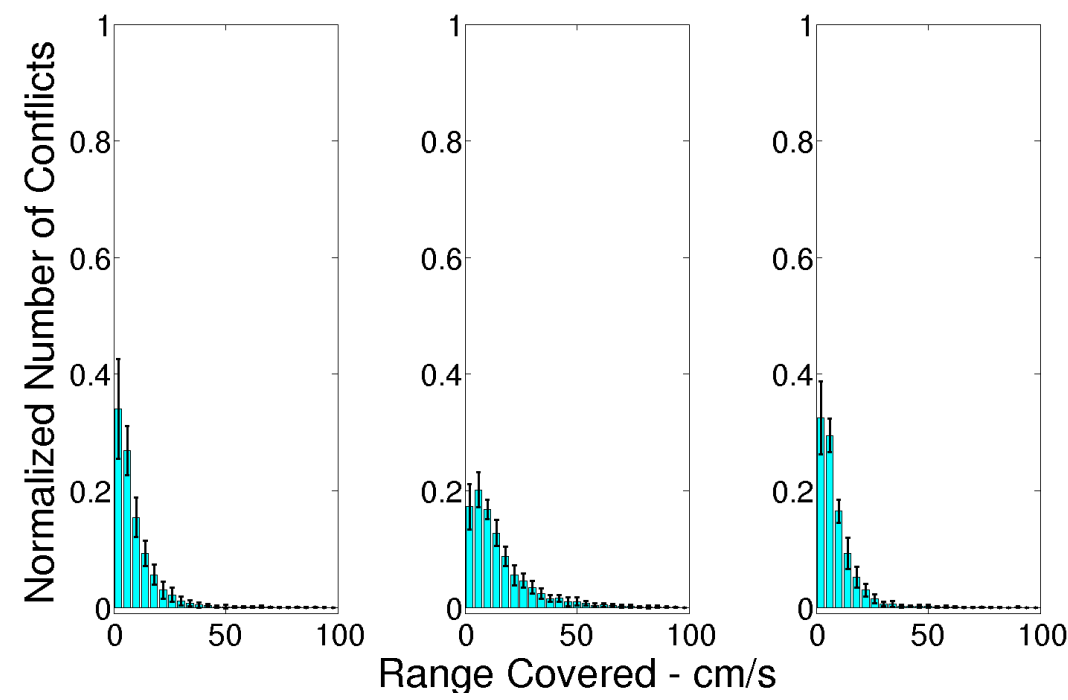

Figure 5.67: The $\Delta V$ distribution for three influential neighbors and $\delta_{\text {attraction }}=$ infinity for the 15-0 giant danio data set under the body-fixed coordinate system with the variable speed maneuver. 


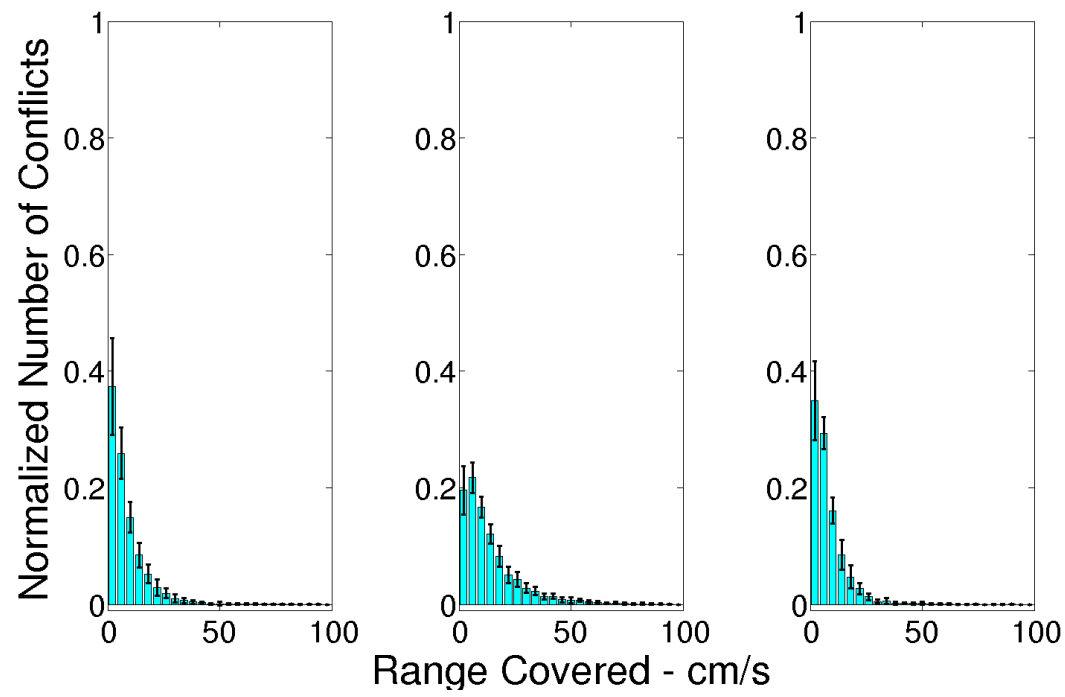

Figure 5.68: The $\Delta V$ distribution for seven influential neighbors and $\delta_{\text {attraction }}=$ infinity for the 15-0 giant danio data set under the body-fixed coordinate system with the variable speed maneuver.
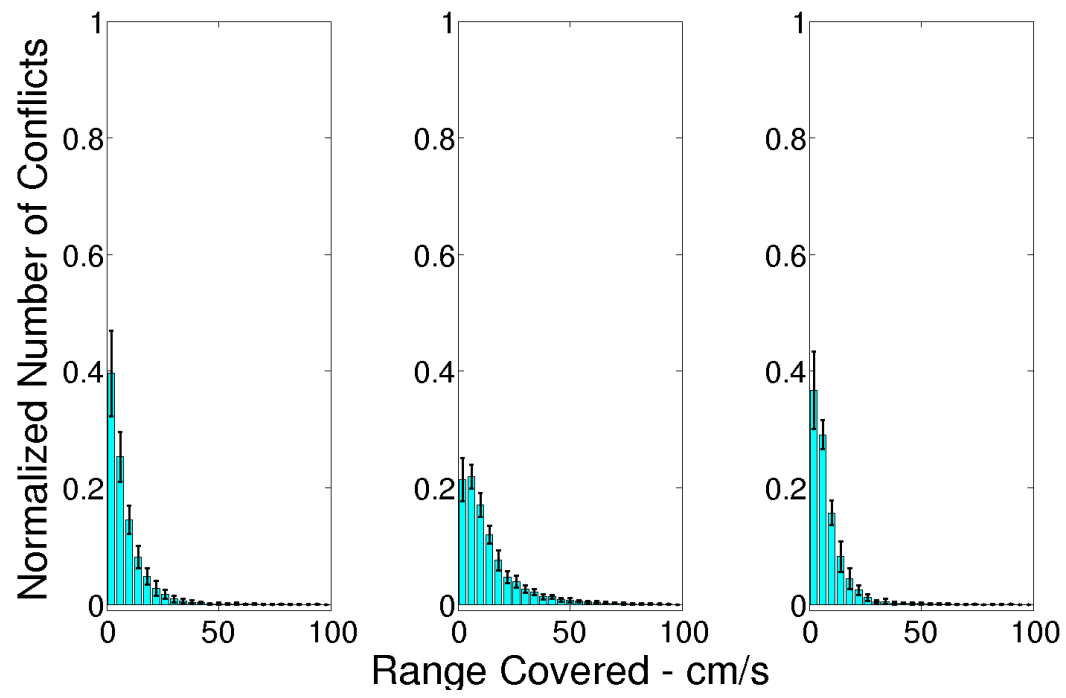

Figure 5.69: The $\Delta V$ distribution for the maximum number of influential neighbors and $\delta_{\text {attraction }}=$ infinity for the 15-0 giant danio data set under the body-fixed coordinate system with the variable speed maneuver. 


\subsection{Barn Swallows}

Six sets of barn swallow data were analyzed. The number of swallows in the field of view changed with each data set, as did the number of frames recorded. For this reason, the maximal number of influential nearest neighbors had different ranges (if only three swallows were in the field of view, at most only two nearest neighbors were considered). The maximum sensing range was chosen from the set of $1.5 \mathrm{~m}, 3 \mathrm{~m}, 5 \mathrm{~m}$, or infinity. All data sets were recorded at 100 frames per second. Table 5.1 outlines the barn swallow data sets. The roofs presented a problem for the two dimensional algorithm considered here. The algorithm, in either coordinate frame, wants to move the animal around the roofs. In reality, the swallow can simply fly over the roof, a motion not allowed in the current collision cone algorithm. In Trial02-2012 and Trial03-2012, the roofs were not considered an obstacle, and the data was analyzed as if the roofs were not present. This situation was also the case for Trial05-2012 in which a swallow were flying close to the ground. In this case, neglecting the obstacle (the ground) would be an extreme simplification of the situation and would not likely generate reasonable results, so Trial05-2012 was not analyzed.

The value of $d_{\text {sep }}$ used for calculating cones with other swallows was 0.4 meters. For the silos, the value of $d_{\text {sep }}$ was 1.95 meters, which is the radius of the silo plus a wingspan. The value of $d_{\text {sep }}$ for the truck obstacle was set to $4 \mathrm{~m}$ to account for the width of the truck. The maximum velocity used in the algorithm was $32 \mathrm{~m} / \mathrm{s}$ to correspond with the maxium velocity of a typical barn swallow. The bin range for each distribution was $2 \mathrm{~m} / \mathrm{s}$. The global coordinate system results were examined first, followed by the results from the body coordinate system. For each coordinate system, the constant and variable speed maneuvers were considered.

Trial03-2012 had two barn swallows in the field of view along with a moving truck. The first swallow, shown in blue in Figure 5.70, did not experience any conflicts. The second swallow, shown in green, experienced three consecutive conflicts with the truck at the beginning of the sequence and experienced six more at the end of the sequence. As can be seen from Figure 5.70, the second swallow did not begin to avoid the truck until the middle of the segment. No conflict occurring during this time indicates, that in this 


\begin{tabular}{l|cccc}
\hline Trial & No. of Frames & No. of Swallows & Obstacles & Influential Neighbors \\
\hline Flock & 475 & 23 & None & 3,7, All \\
Trial03 & 375 & 12 & None & 3,7 , All \\
Trial04 & 301 & 3 & 2 Silos & 3 \\
Trial02-2012 & 151 & 3 & Roof, 2 Silos & 3 \\
Trial03-2012 & 280 & 2 & Roof, Truck & 2 \\
Trial05-2012 & 251 & 2 & Ground & 2 \\
\hline
\end{tabular}

Table 5.1: Table outline the different barn swallow data sets.

instance, swallow two may not have used collision cones to avoid the truck.

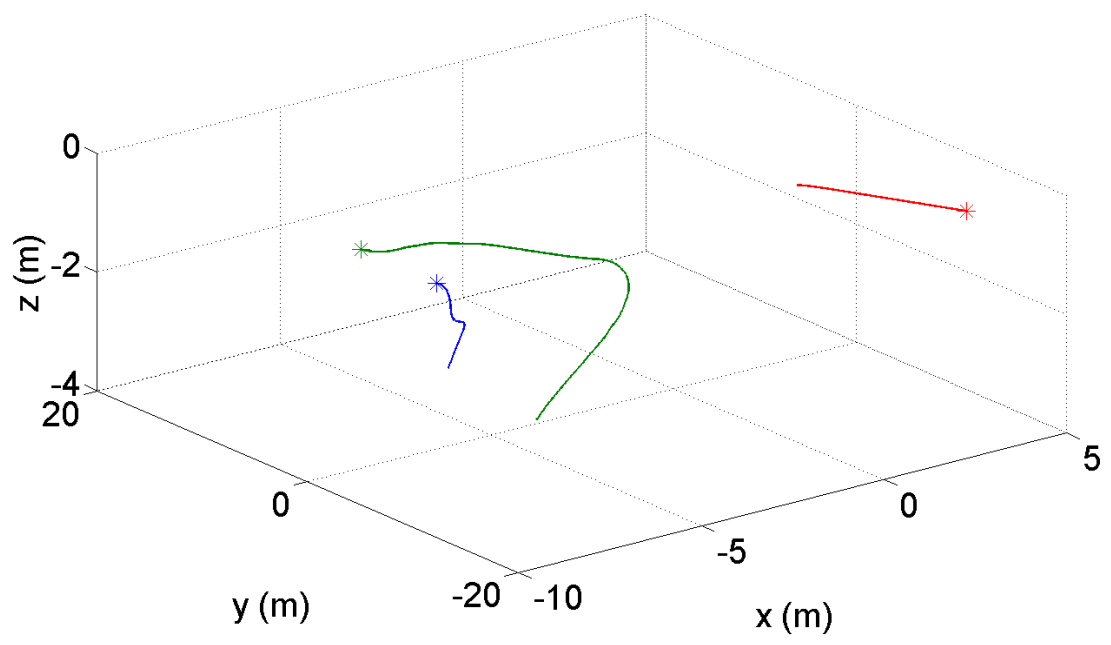

Figure 5.70: The trajectory data for Trial03-2012 showing the two barn swallows and the truck. The stars mark the beginning of the trajectories.

\subsubsection{Global Coordinates}

In the global coordinate system, first the constant speed maneuver was analyzed and then the variable speed maneuver. 


\section{Constant Speed Maneuver}

The first data set to be analyzed was the Flock. First the $\delta_{\text {attraction }}$ was held at infinity while the number of influential nearest neighbors was varied. As can be seen in Figures 5.71-5.73, varying the number of influential nearest neighbors did not appear to have any effect on the correlation distributions. Each plot showed that a little less than $80 \%$ of the time, the swallow's next velocity vector was less than $2 \mathrm{~m} / \mathrm{s}$ away from the optimal velocity vector as predicted by the DRCA algorithm. Next, the number of influential nearest neighbors was held constant at seven, and the maximum sensing range was varied (Figures 5.745.76). Again, the correlation between actual and predicted response was near $80 \%$ for speed within $2 \mathrm{~m} / \mathrm{s}$, and the variation between variations in numbers of nearest neighbors relative to variations in sensing range was not large enough to indicate which parameters have more effect on correlation.

The next set of data to be examined was Trial03. When holding $\delta_{\text {attraction }}$ at infinity and varying the number of influential nearest neighbors (Figures 5.77-5.79), the distributions did not change much. The distributions showed fairly good results with the maximum $\Delta V$ being six meters per second, which was less than $20 \%$ of the maximum velocity. Holding the number of influential nearest neighbors constant at seven and varying $\delta_{\text {attraction }}$ resulted in Figures 5.80-5.82. Lowering the $\delta_{\text {attraction }}$ produced lower correlation results, with the first bin being less than $40 \%$. But overall, the average $\Delta V$ for any conflict was no more than 6 $\mathrm{m} / \mathrm{s}$.

Next the two data sets with the silo obstacles were considered. All the distributions for the Trial04 data set indicated results that fit the algorithm well (Figures 5.83-5.86). These distributions represented results for three influential neighbors and variations in $\delta_{\text {attraction }}$. The best distribution was the one for $\delta_{\text {attraction }}$ equal to $3 \mathrm{~m}$ with the $\Delta V$ value being less than $2 \mathrm{~m} / \mathrm{s}$ almost $90 \%$ of the time. The distributions for Trial02-2012 were shown in Figures 5.87-5.90. The results were not as strongly correlated as the previous data set with the pair of silo obstacles. This tendency could be a result of ignoring the roof. The worst results were from the distribution with the maximum sensing range set to $1.5 \mathrm{~m}$. 


\section{Variable Speed Maneuver}

Now the variable speed maneuver were next examined for the barn swallow data. First, the two data sets without static obstacles were considered. The $\Delta V$ distributions for the Flock data sets are shown in Figures 5.91-5.96. When fixing the maximum sensing range at infinity, varying the number of influential neighbors did not result in a significant change in the distributions. Similarly, varying the maximum sensing range while keeping the number of influential neighbors fixed at seven did not change the distributions.

The $\Delta V$ distributions for the Trial03 data set, Figures 5.97-5.102, all show that the maximum $\Delta V$ value was $4 \mathrm{~cm} / \mathrm{s}$. Varying the number of influential neighbors did not result in a significant change in the distributions (Figures 5.97-5.99) but the distributions decreased for $\delta_{\text {atrraction }}$ less than infinity (Figures 5.100-5.102). Varying the maximum sensing range while keeping the number of influential neighbors fixed at seven did not change the distributions.

Next the data sets Trial04 and Trial02-2012 were analyzed for the global coordinate frame variable speed maneuver. The distributions for Trial04 were shown in Figures 5.103-5.106. The best distribution was produced for $\delta_{\text {attraction }}=3 \mathrm{~m}$. The distributions for Trial02-2012 are shown in Figures 5.107-5.110. The results were once again worse in comparison to the previous data set Trial04. This drop in correlation could be a result of ignoring the roof that was present in Trial02-2012. The worst results were again from the distribution with the maximum sensing range set to $1.5 \mathrm{~m}$.

\subsubsection{Body Coordinates}

Now the data sets were looked at from the body coordinate point of view.

\section{Constant Speed Maneuver}

First, the constant speed maneuver was explored. The Flock data set, Figures 5.111-5.113, showed the worst results for $\delta_{\text {attraction }}$ fixed at infinity when seven influential nearest neighbors were used. Overall, the results for the Flock data set have highly correlated results, greater than $80 \%$ of the time $\Delta V$ being no more than $2 \mathrm{~m} / \mathrm{s}$. When varying the maximum 
sensing range, Figures 5.114-5.116, the best distribution occurred for $\delta_{\text {attraction }}=5 \mathrm{~m}$. In Figure 5.116, which had $\delta_{\text {attraction }}=1.5 \mathrm{~m}$ and seven influential neighbors, a second peak occurred with a $10-12 \mathrm{~m} / \mathrm{s}$ bin that almost reached to $40 \%$. This second peak is the product of the high standard deviation seen in each of the other bins. The high standard deviation leads us to believe that this set of parameters was not a good representation for the barn swallows of this particular data set.

The Trial03 data set, Figures 5.117-5.119, showed the worst results for a $\delta_{\text {attraction }}$ fixed at infinity with three influential nearest neighbors, with $\Delta V$ being less than $2 \mathrm{~m} / \mathrm{s}$ about $70 \%$ of the time. When the number of influential nearest neighbors was seven or all, and $\delta_{\text {attraction }}=$ infinity, $\Delta V$ was less than $2 \mathrm{~m} / \mathrm{s}$ about $80 \%$ of the time. When varying the maximum sensing range, Figures 5.120-5.122, the best distribution occured when $\delta_{\text {attraction }}=5 \mathrm{~m}$.

Next the two data sets with the silo obstacles were analyzed in the body coordinate frame for the constant speed maneuver. All the distributions for the Trial04 data set produced results that fit the algorithm well, (Figures 5.123-5.126). These distributions utilized three influential neighbors and varied $\delta_{\text {attraction. }}$. The distribution with the highest correlation was the one for $\delta_{\text {attraction }}=3 \mathrm{~m}$ where $\Delta V$ was less than $2 \mathrm{~m} / \mathrm{s}$ almost $80 \%$ of the time. The distributions for Trial02-2012 were shown in Figures 5.127-5.130. The results were not as well correlated in comparison to the previous data set, Trial04, for the constant speed maneuver (Figures 5.123-5.126) with the pair of silo obstacles. This difference could be a result of ignoring the roof. The best results are from the distribution with the maximum sensing range set to $5 \mathrm{~m}$, where $\Delta V$ was less than $2 \mathrm{~m} / \mathrm{s}$ approximately $75 \%$ of the time.

\section{Variable Speed Maneuver}

The final senario to be analyzed was the body coordinate variable speed maneuver. The data sets without static obstacles were examined first. Figures 5.131-5.136 showed the $\Delta V$ distributions for the Flock data set. Varying the number of influential neighbors did not result in a change in the distributions when the maximum sensing range was set at infinity. Varying the maximum sensing range while keeping the number of influential neighbors fixed at seven did not lead to enough of a change in the distributions enough to determine which $\delta_{\text {attraction }}$ 
produced better results. A second peak in Figure 5.136, which has $\delta_{\text {attraction }}=1.5 \mathrm{~m}$ and seven influential neighbors, indicated that these set of parameters might not be the best fit for the barn swallows. This second peak was also seen in Figure 5.116 for the body coordinate constant speed maneuver, but the peak and standard deviations were less in the variable speed maneuver scenario.

The $\Delta V$ distributions for the Trial03 data set, Figures 5.137-5.142, all showed that the maximum $\Delta V$ value was $4 \mathrm{~cm} / \mathrm{s}$. Varying the number of influential neighbors did not increase the correlation of the distributions (Figures 5.137-5.139), but the distributions decreased for $\delta_{\text {atrraction }}$ less than infinity (Figures 5.140-5.142). Varying the maximum sensing range while keeping the number of influential neighbors fixed at seven did not change the distributions (Figures 5.138, 5.140-5.142).

The distributions for Trial04 with the body coordinate frame and variable speed maneuver are shown in Figures 5.143-5.146. None of the distributions was significantly better than the others in this scenario. The distributions for Trial02-2012 were shown in Figures 5.147-5.150. The results for the the maximum sensing range at $5 \mathrm{~m}$ and infinity were about the same as the distributions produced by Trial04, but as $\delta_{\text {attraction }}$ decreased so did the correlation of the algorithm and data. The least correlated results were from the distribution with the maximum sensing range set to $1.5 \mathrm{~m}$.

\subsubsection{Discussion of Barn Swallow Data Analysis}

The variable speed results for both coordinate systems seemed to produce slightly better results than the constant speed results. Further exploration is needed to determine if the variable speed maneuver is worth pursuing due to its computationally heavier load. When the roofs were ignored, the distributions showed less correlation. A three-dimensional collision cone algorithm is needed to study the effects of the roof. The silo obstacles did not affect the results of the distributions, suggesting that the barn swallows were avoiding these static obstacle in the same way as they were avoiding other barn swallows. The comparison of the analyses of the global and body coordinate frame choices did not result in an appreciable difference in correlation between algorithm and biological data. 


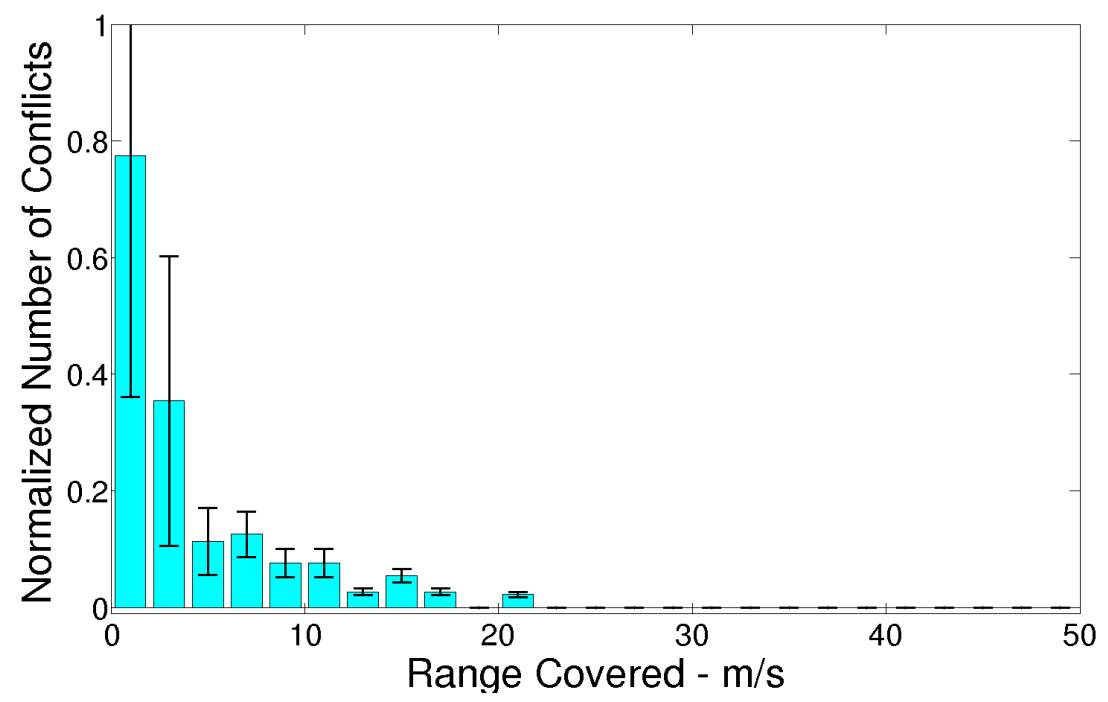

Figure 5.71: The $\Delta V$ distribution for three influential neighbors and $\delta_{\text {attraction }}=$ infinity for the Flock barn swallow data set under the global coordinate system with the constant speed maneuver. 


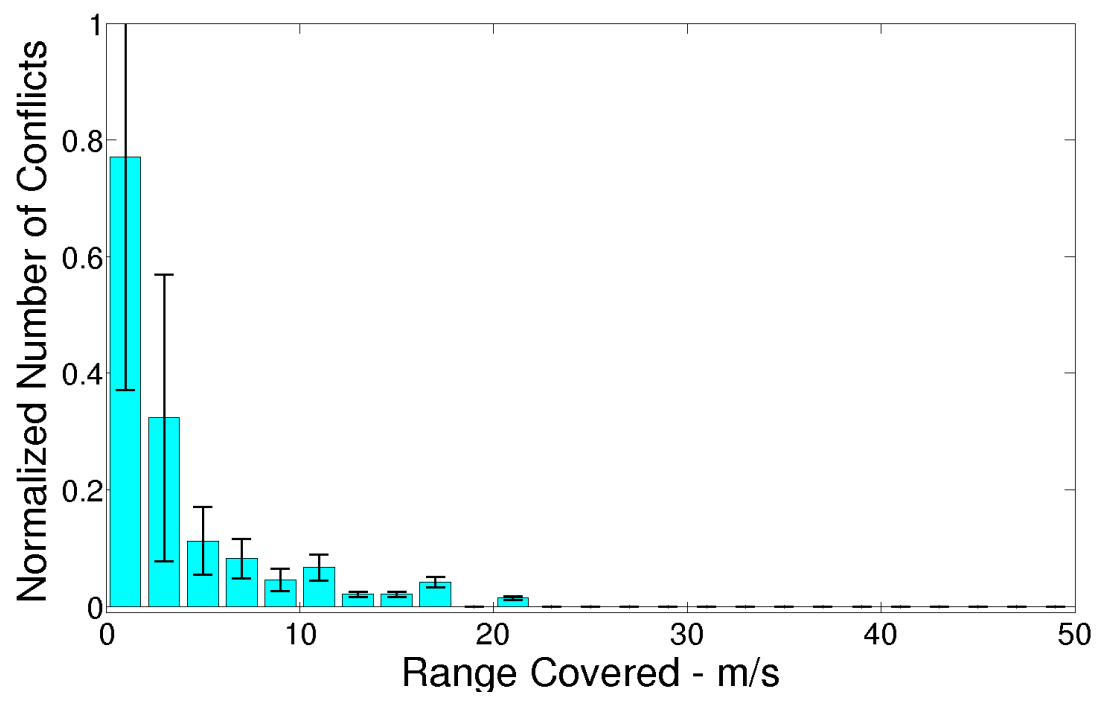

Figure 5.72: The $\Delta V$ distribution for seven influential neighbors and $\delta_{\text {attraction }}=$ infinity for the Flock barn swallow data set under the global coordinate system with the constant speed maneuver.

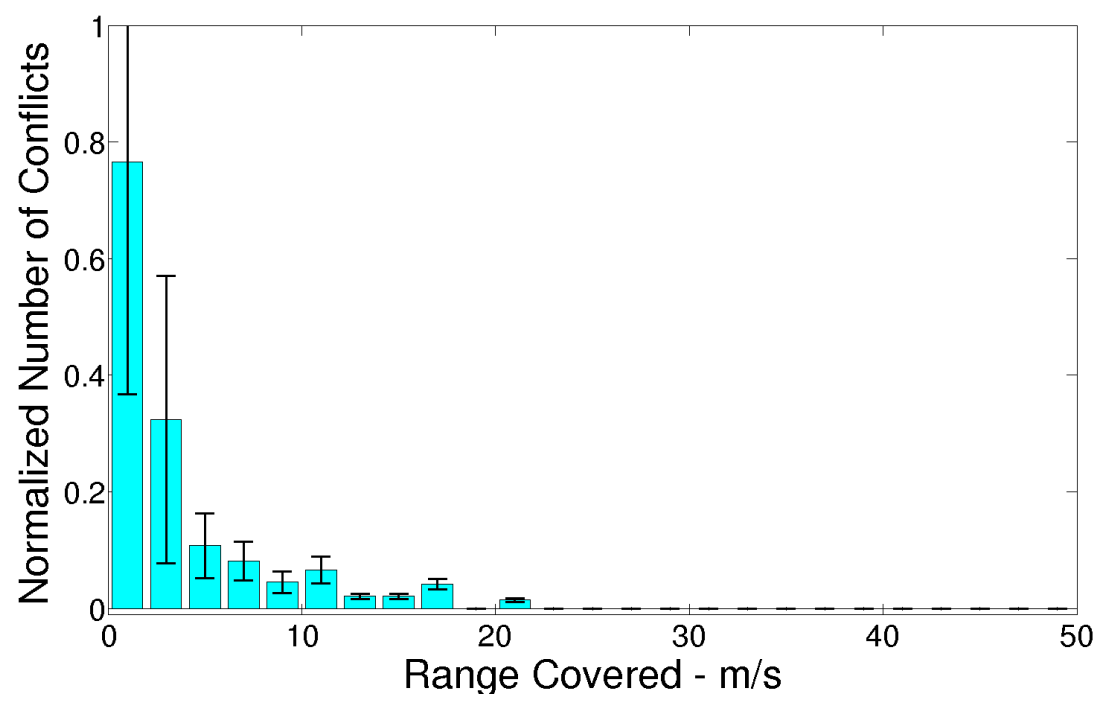

Figure 5.73: The $\Delta V$ distribution for the maximum number of influential neighbors and $\delta_{\text {attraction }}=$ infinity for the Flock barn swallow data set under the global coordinate system with the constant speed maneuver. 


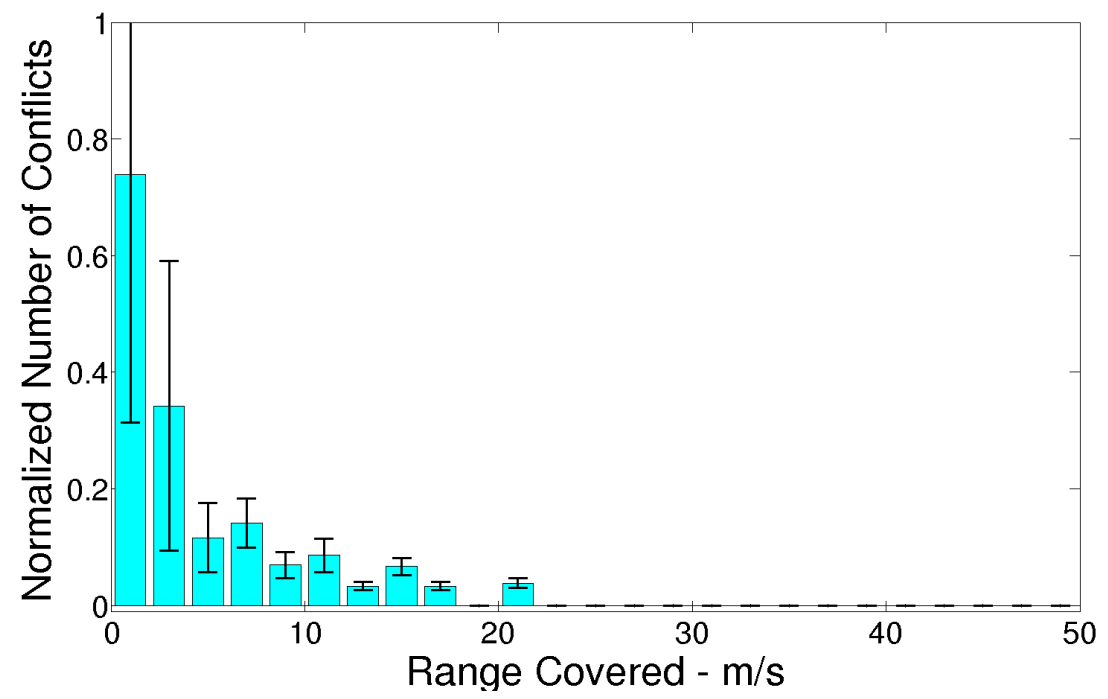

Figure 5.74: The $\Delta V$ distribution for seven influential neighbors and $\delta_{\text {attraction }}=5 \mathrm{~m}$ for the Flock barn swallow data set under the global coordinate system with the constant speed maneuver.

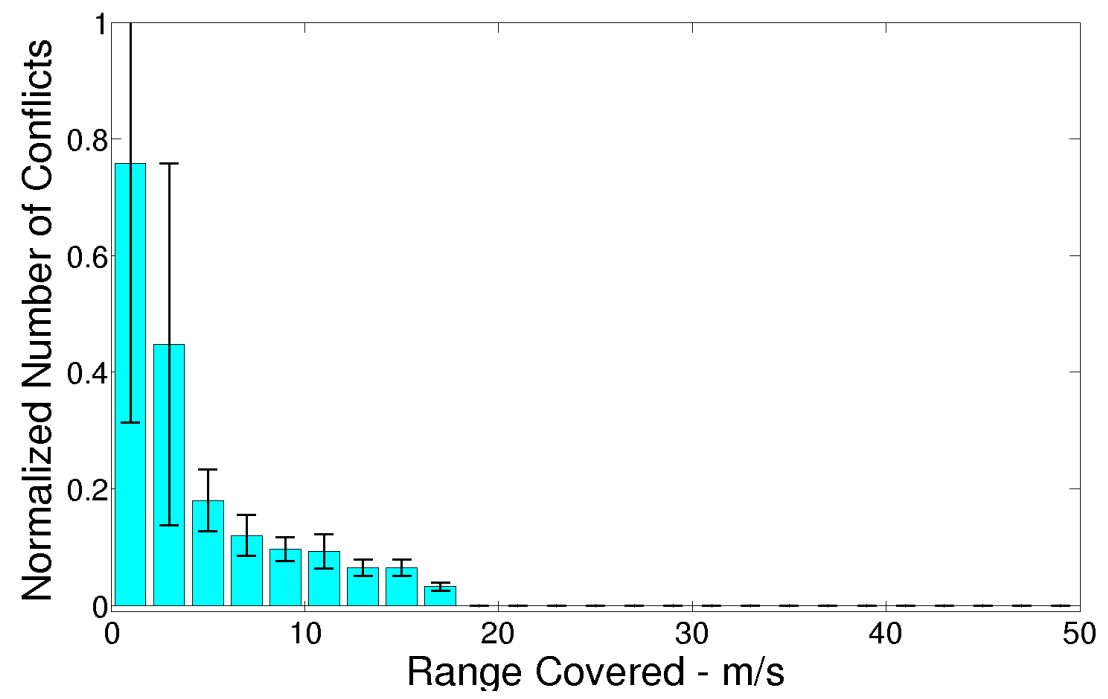

Figure 5.75: The $\Delta V$ distribution for seven influential neighbors and $\delta_{\text {attraction }}=3 \mathrm{~m}$ for the Flock barn swallow data set under the global coordinate system with the constant speed maneuver. 


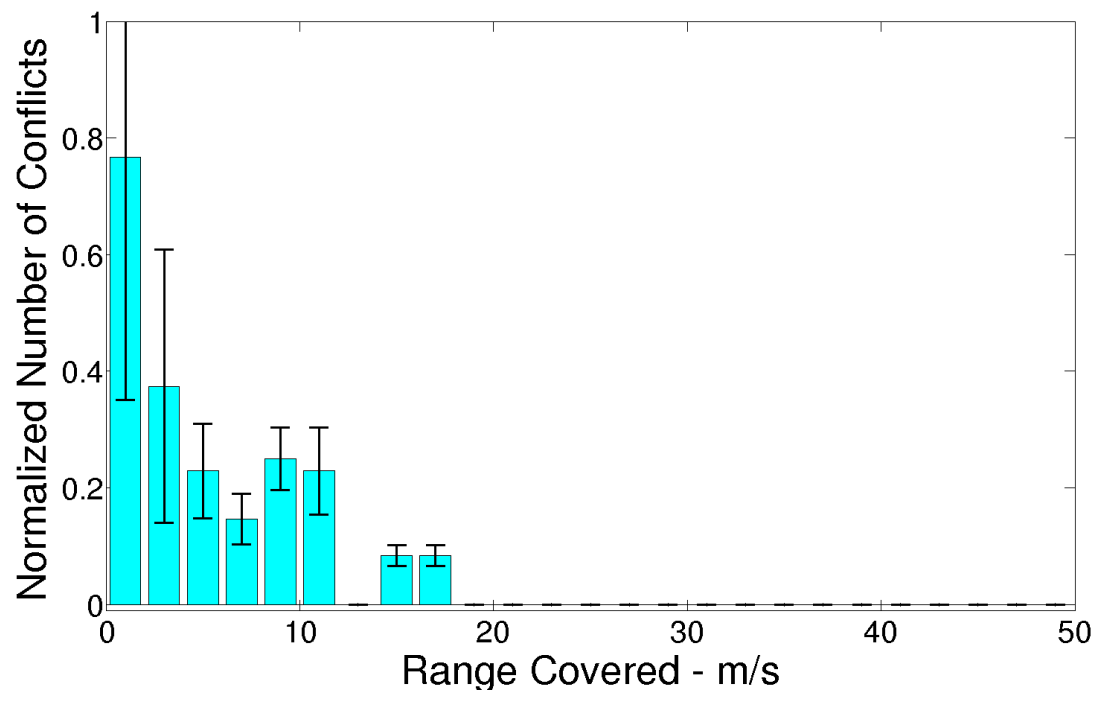

Figure 5.76: The $\Delta V$ distribution for seven influential neighbors and $\delta_{\text {attraction }}=1.5 \mathrm{~m}$ for the Flock barn swallow data set under the global coordinate system with the constant speed maneuver.

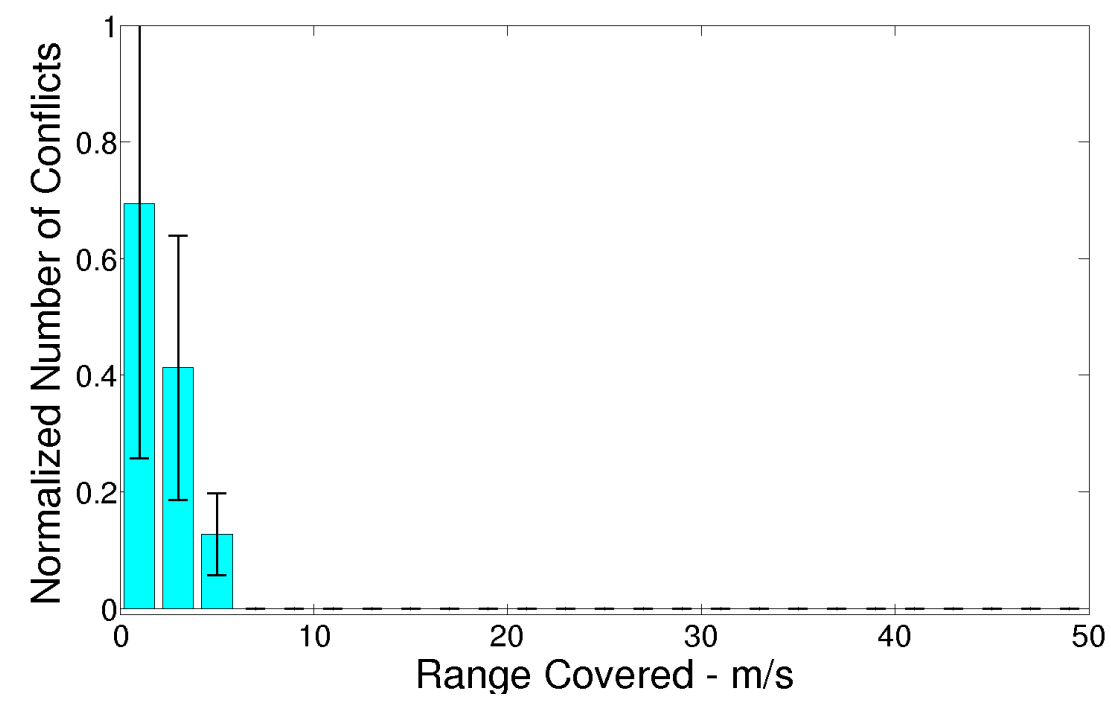

Figure 5.77: The $\Delta V$ distribution for three influential neighbors and $\delta_{\text {attraction }}=$ infinity for the Trial03 barn swallow data set under the global coordinate system with the constant speed maneuver. 


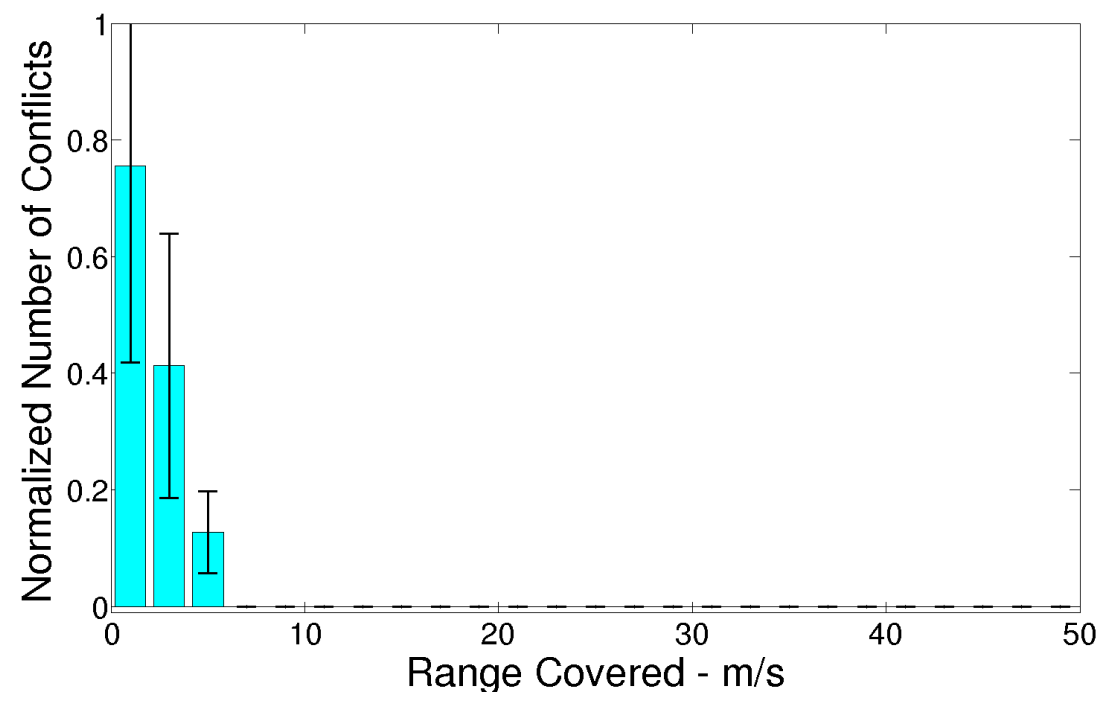

Figure 5.78: The $\Delta V$ distribution for seven influential neighbors and $\delta_{\text {attraction }}=$ infinity for the Trial03 barn swallow data set under the global coordinate system with the constant speed maneuver.

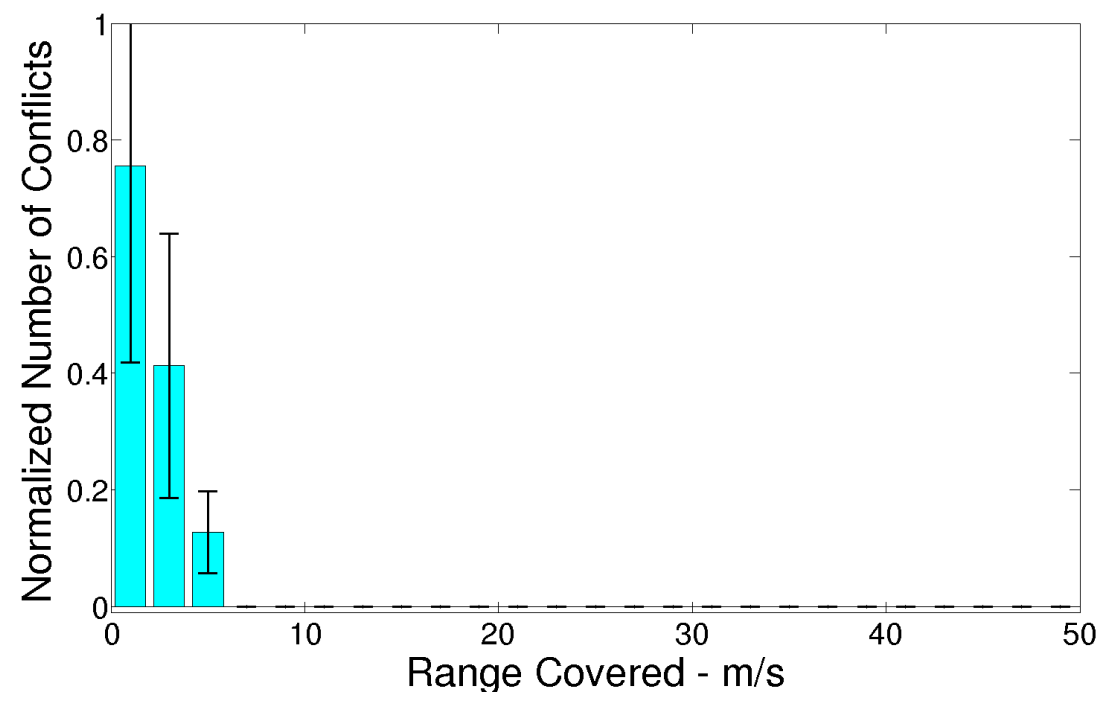

Figure 5.79: The $\Delta V$ distribution for the maximum number of influential neighbors and $\delta_{\text {attraction }}=$ infinity for the Trial03 barn swallow data set under the global coordinate system with the constant speed maneuver. 


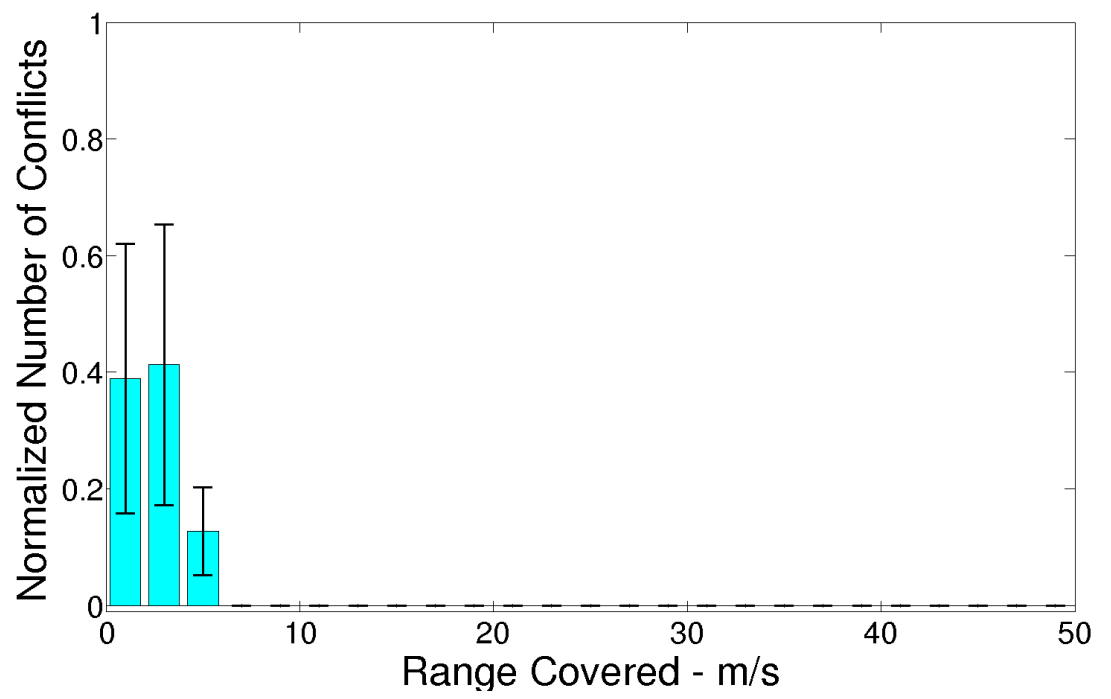

Figure 5.80: The $\Delta V$ distribution for seven influential neighbors and $\delta_{\text {attraction }}=5 \mathrm{~m}$ for the Trial03 barn swallow data set under the global coordinate system with the constant speed maneuver.

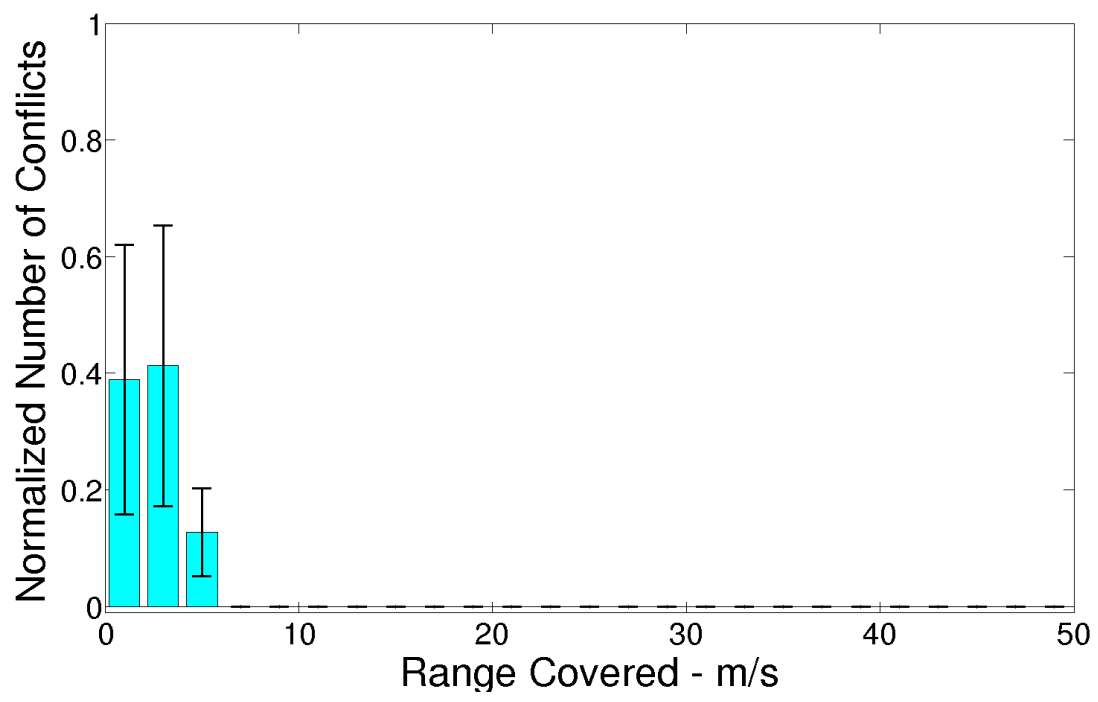

Figure 5.81: The $\Delta V$ distribution for seven influential neighbors and $\delta_{\text {attraction }}=3 \mathrm{~m}$ for the Trial03 barn swallow data set under the global coordinate system with the constant speed maneuver. 


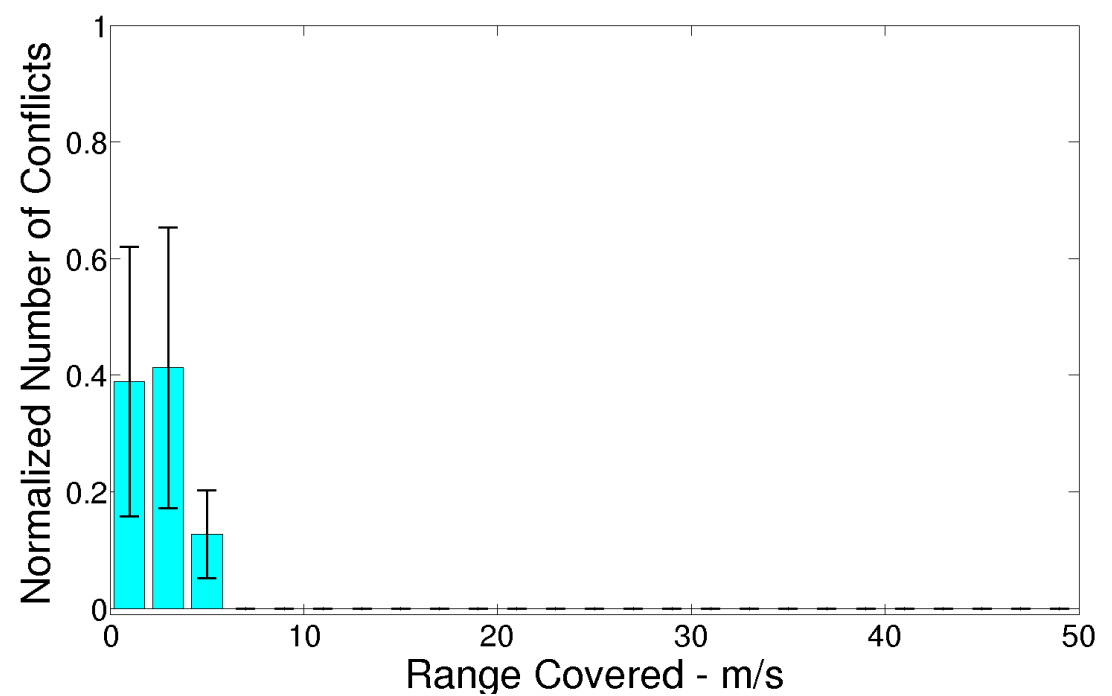

Figure 5.82: The $\Delta V$ distribution for seven influential neighbors and $\delta_{\text {attraction }}=1.5 \mathrm{~m}$ for the Trial03 barn swallow data set under the global coordinate system with the constant speed maneuver. 


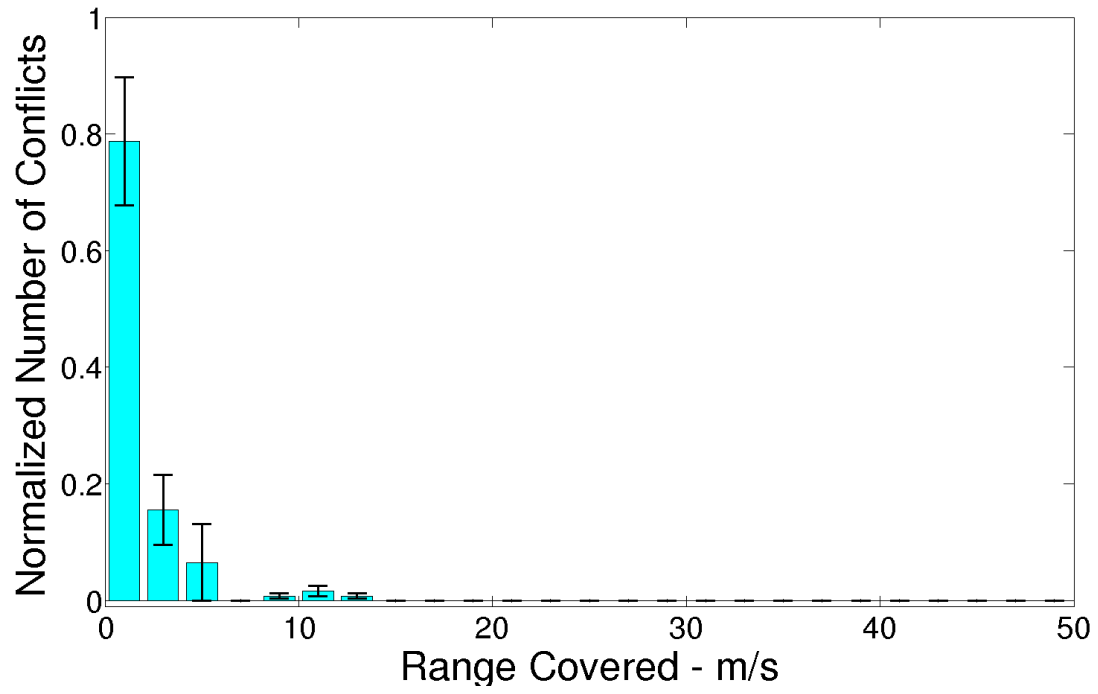

Figure 5.83: The $\Delta V$ distribution for three influential neighbors and $\delta_{\text {attraction }}=$ infinity for the Trial04 barn swallow data set under the global coordinate system with the constant speed maneuver. 


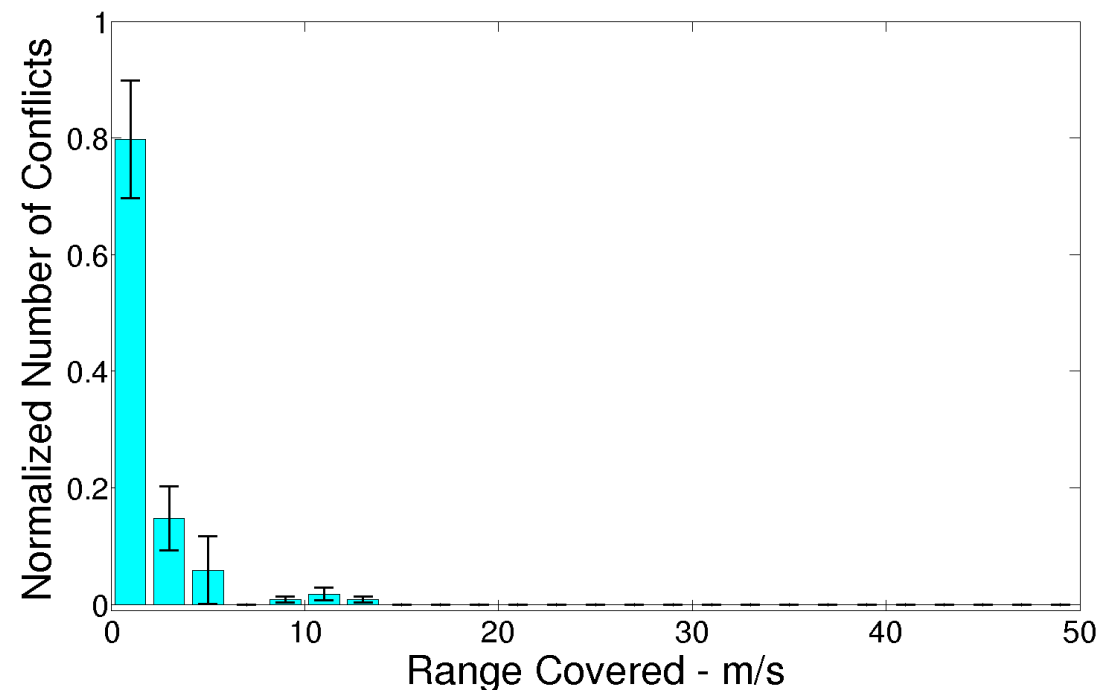

Figure 5.84: The $\Delta V$ distribution for three influential neighbors and $\delta_{\text {attraction }}=5 \mathrm{~m}$ for the Trial04 barn swallow data set under the global coordinate system with the constant speed maneuver.

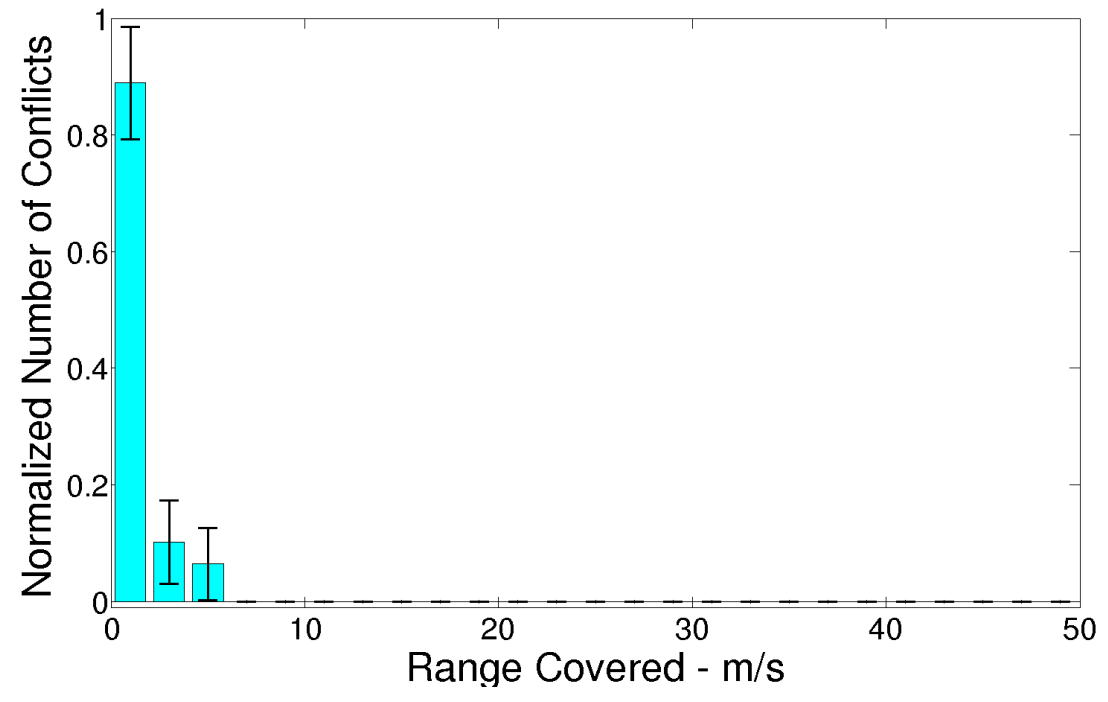

Figure 5.85: The $\Delta V$ distribution for three influential neighbors and $\delta_{\text {attraction }}=3 \mathrm{~m}$ for the Trial04 barn swallow data set under the global coordinate system with the constant speed maneuver. 


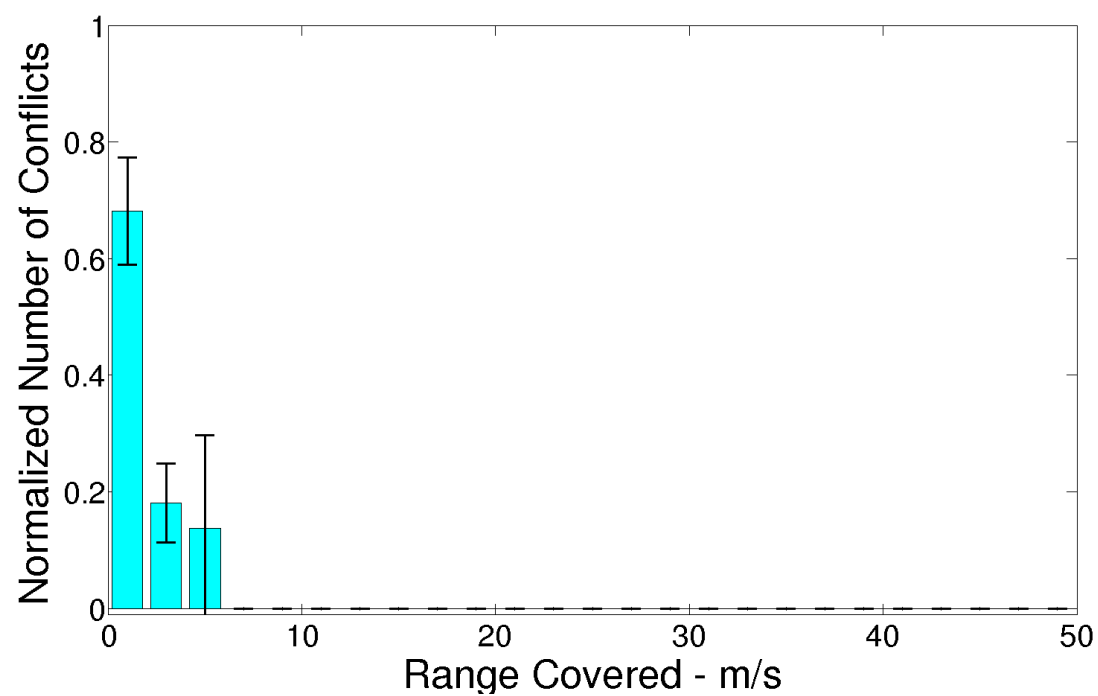

Figure 5.86: The $\Delta V$ distribution for three influential neighbors and $\delta_{\text {attraction }}=1.5 \mathrm{~m}$ for the Trial04 barn swallow data set under the global coordinate system with the constant speed maneuver. 


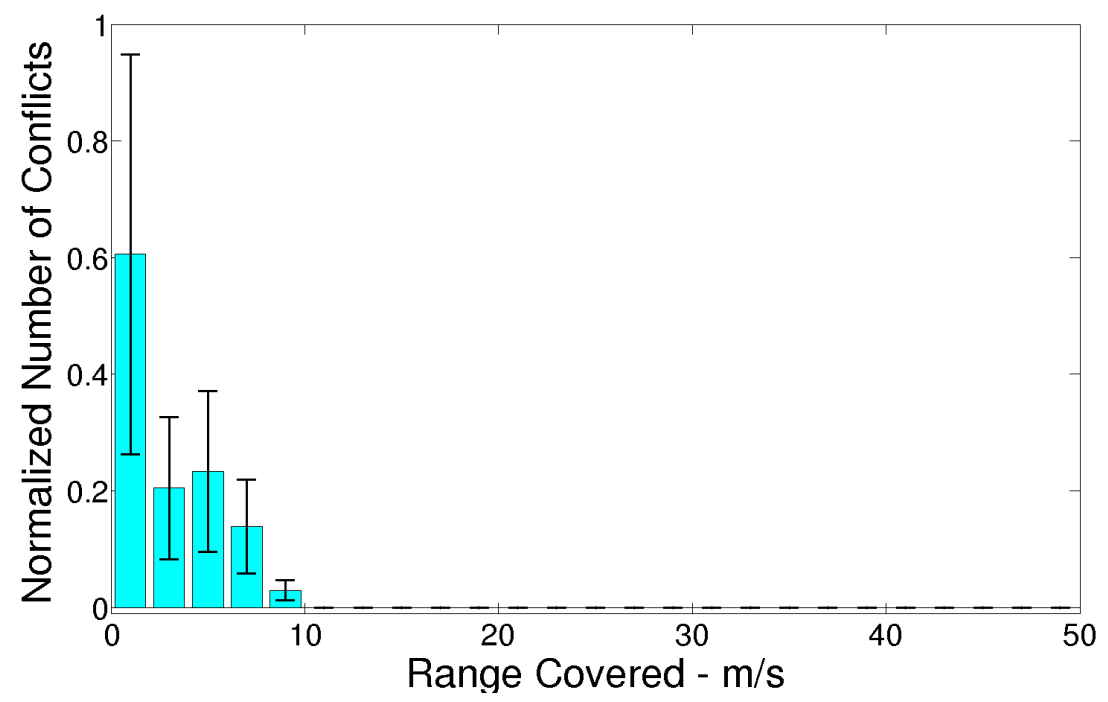

Figure 5.87: The $\Delta V$ distribution for three influential neighbors and $\delta_{\text {attraction }}=$ infinity for the Trial02-2012 barn swallow data set under the global coordinate system with the constant speed maneuver. 


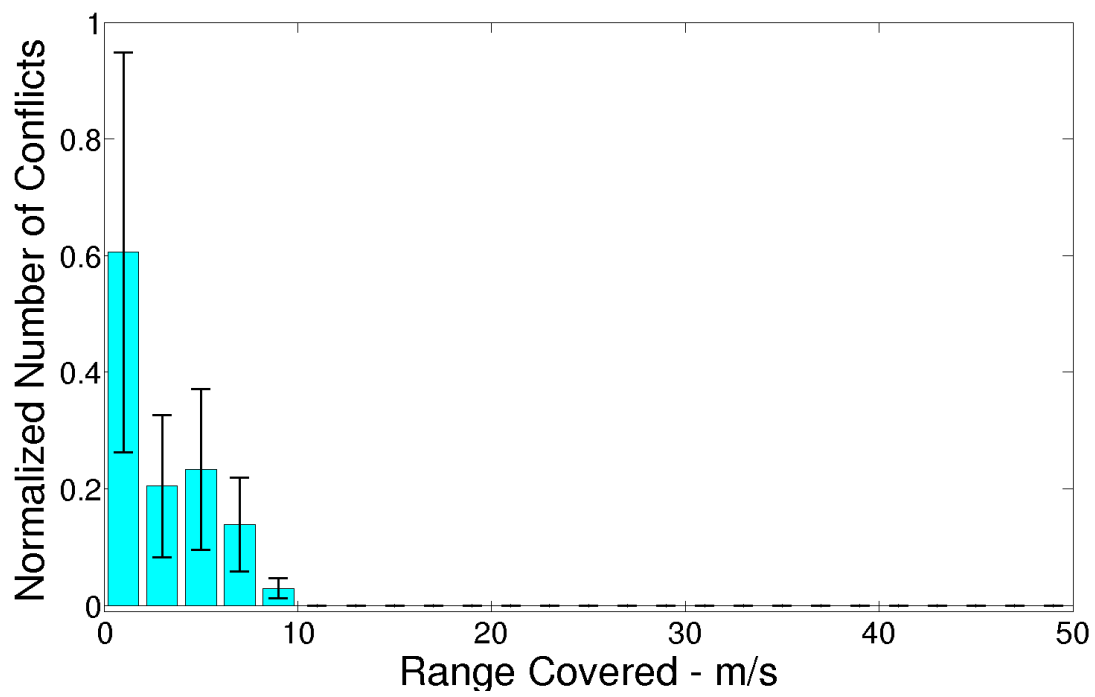

Figure 5.88: The $\Delta V$ distribution for three influential neighbors and $\delta_{\text {attraction }}=5 \mathrm{~m}$ for the Trial02-2012 barn swallow data set under the global coordinate system with the constant speed maneuver.

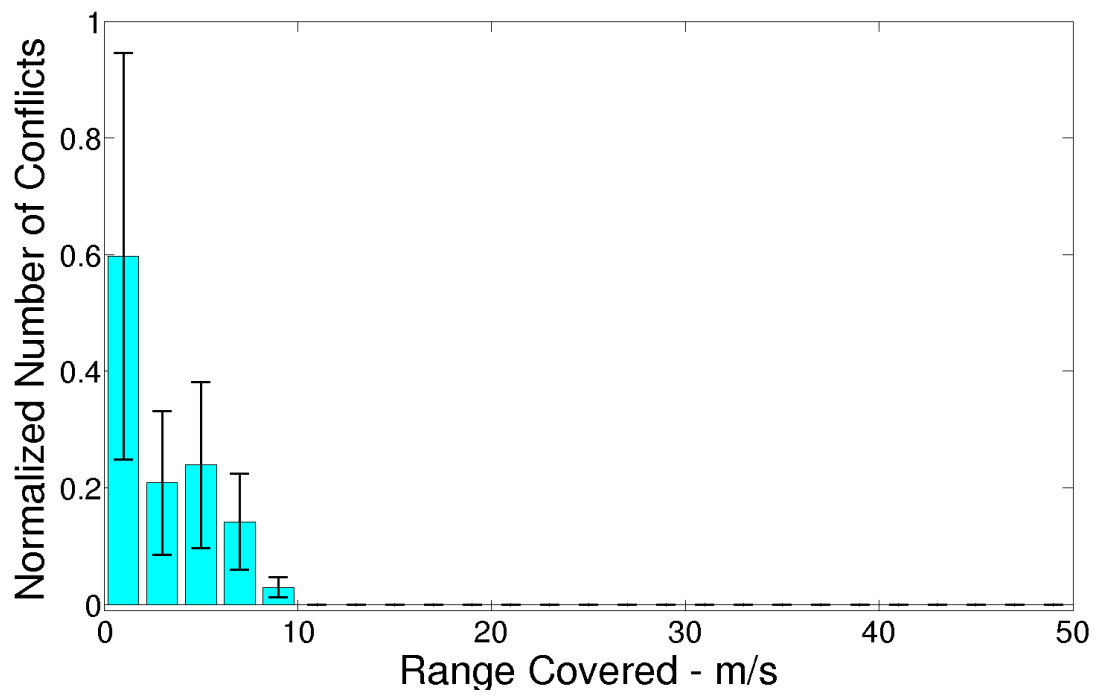

Figure 5.89: The $\Delta V$ distribution for three influential neighbors and $\delta_{\text {attraction }}=3 \mathrm{~m}$ for the Trial02-2012 barn swallow data set under the global coordinate system with the constant speed maneuver. 


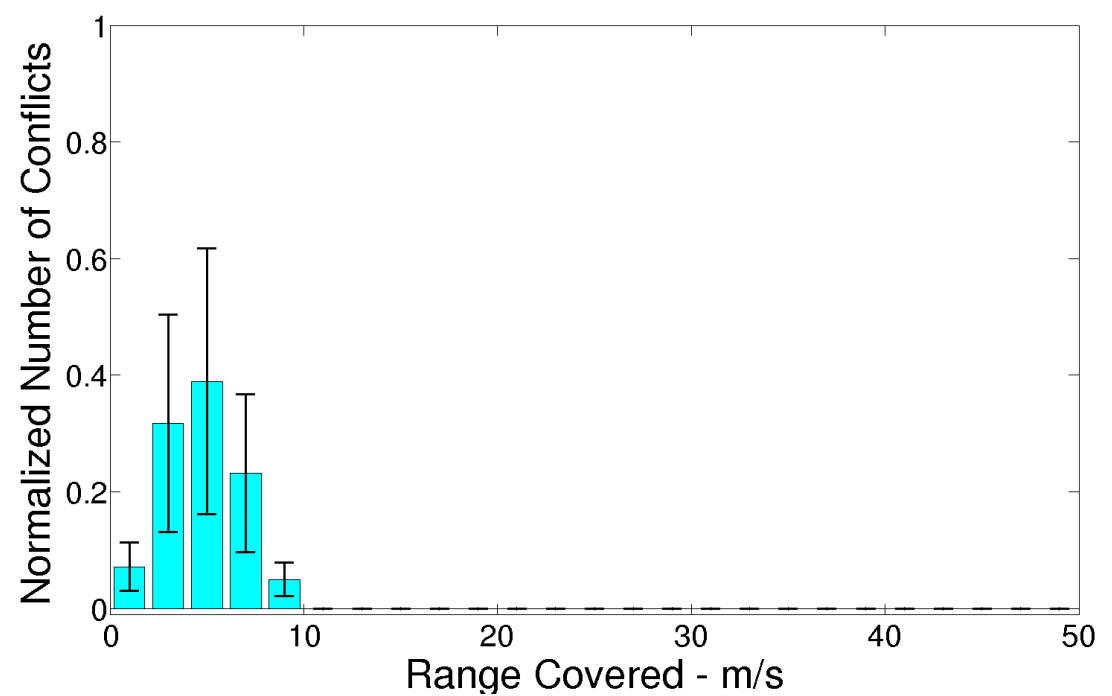

Figure 5.90: The $\Delta V$ distribution for three influential neighbors and $\delta_{\text {attraction }}=1.5 \mathrm{~m}$ for the Trial02-2012 barn swallow data set under the global coordinate system with the constant speed maneuver. 


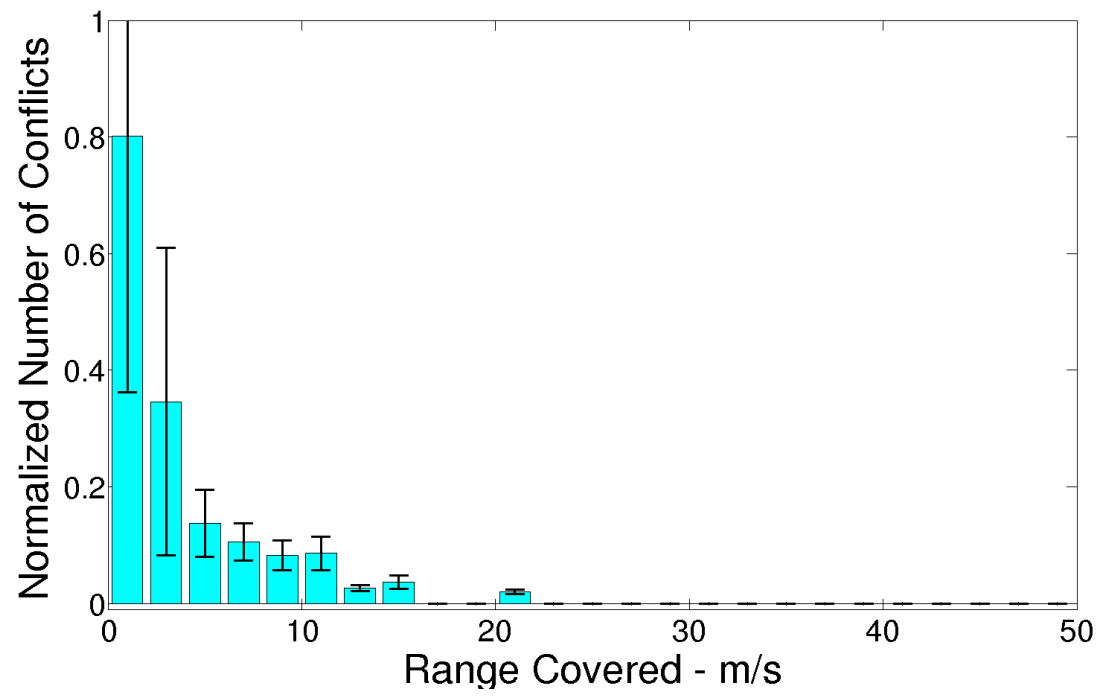

Figure 5.91: The $\Delta V$ distribution for three influential neighbors and $\delta_{\text {attraction }}=$ infinity for the Flock barn swallow data set under the global coordinate system with the variable speed maneuver. 


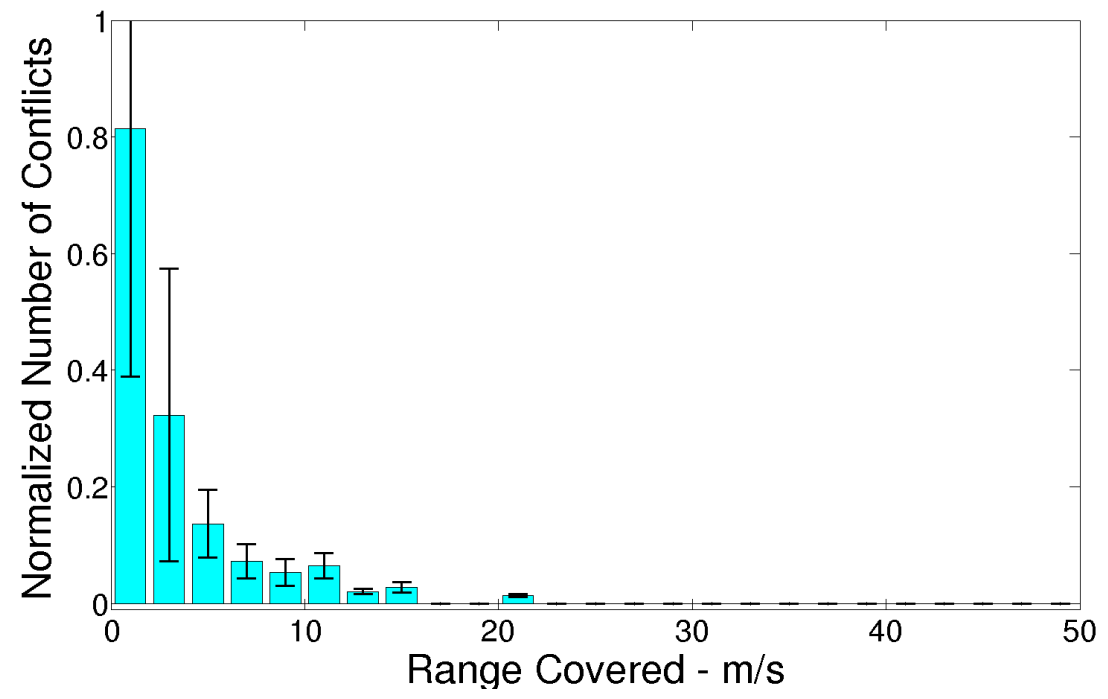

Figure 5.92: The $\Delta V$ distribution for seven influential neighbors and $\delta_{\text {attraction }}=$ infinity for the Flock barn swallow data set under the global coordinate system with the variable speed maneuver.

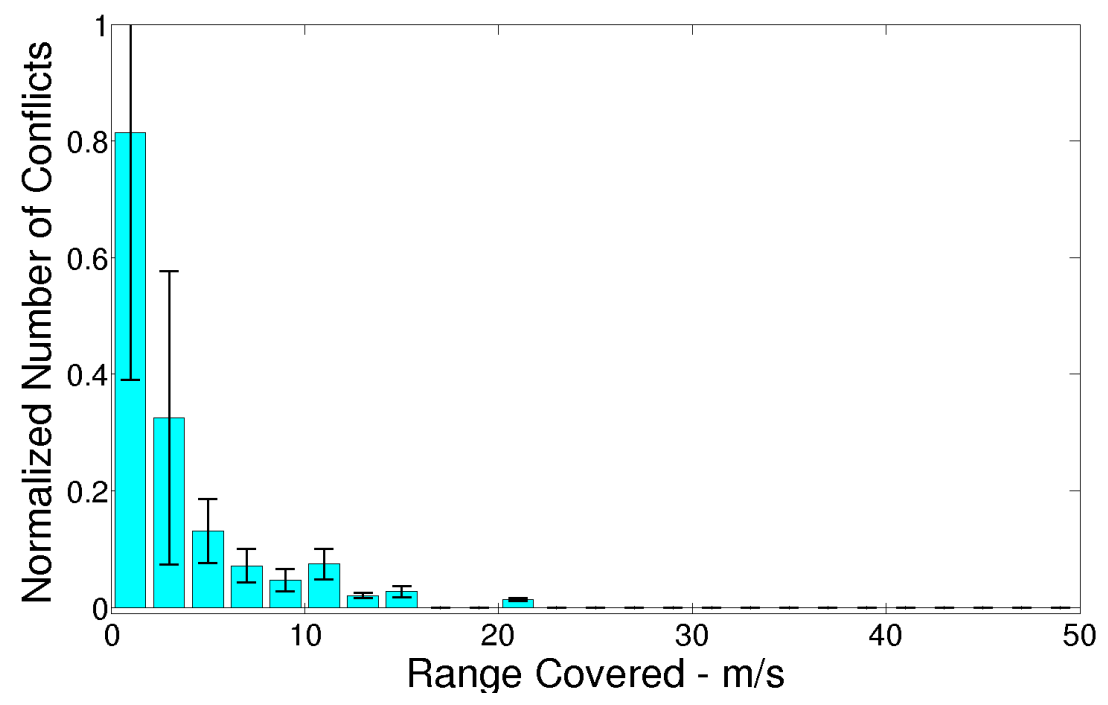

Figure 5.93: The $\Delta V$ distribution for the maximum number of influential neighbors and $\delta_{\text {attraction }}=$ infinity for the Flock barn swallow data set under the global coordinate system with the variable speed maneuver. 


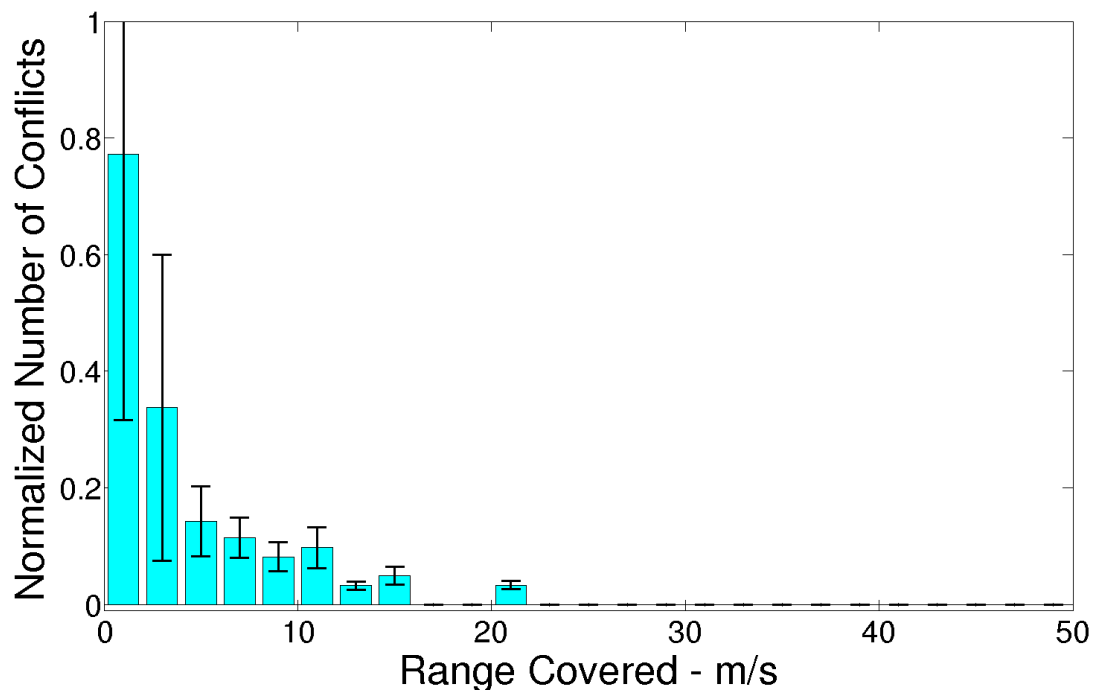

Figure 5.94: The $\Delta V$ distribution for seven influential neighbors and $\delta_{\text {attraction }}=5 \mathrm{~m}$ for the Flock barn swallow data set under the global coordinate system with the variable speed maneuver.

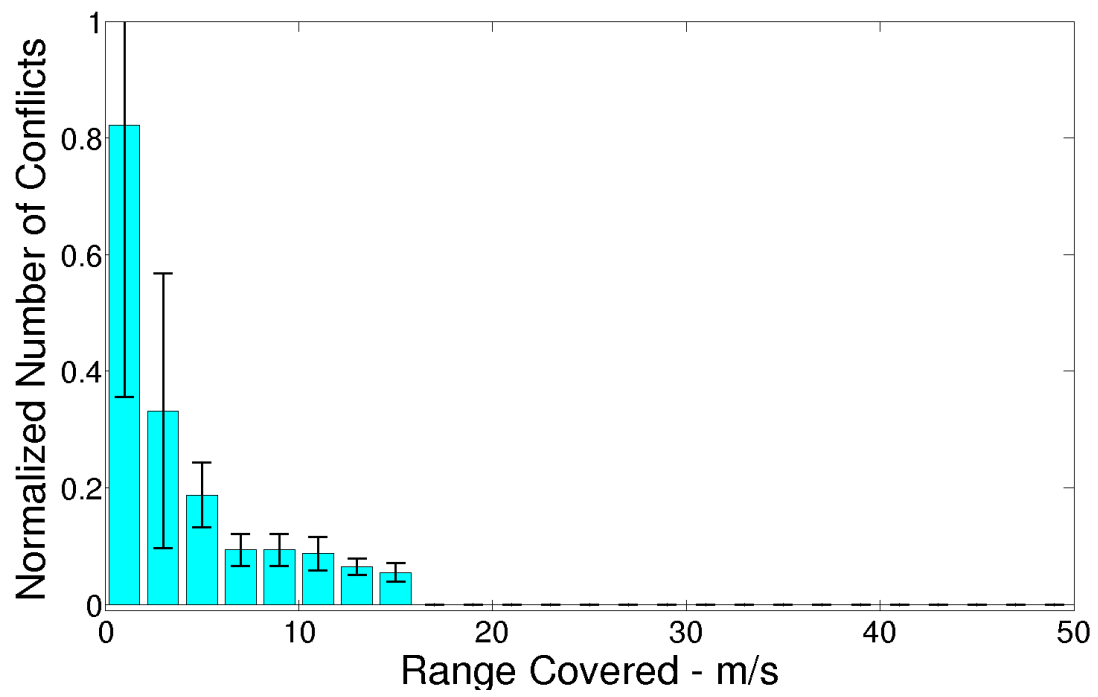

Figure 5.95: The $\Delta V$ distribution for seven influential neighbors and $\delta_{\text {attraction }}=3 \mathrm{~m}$ for the Flock barn swallow data set under the global coordinate system with the variable speed maneuver. 


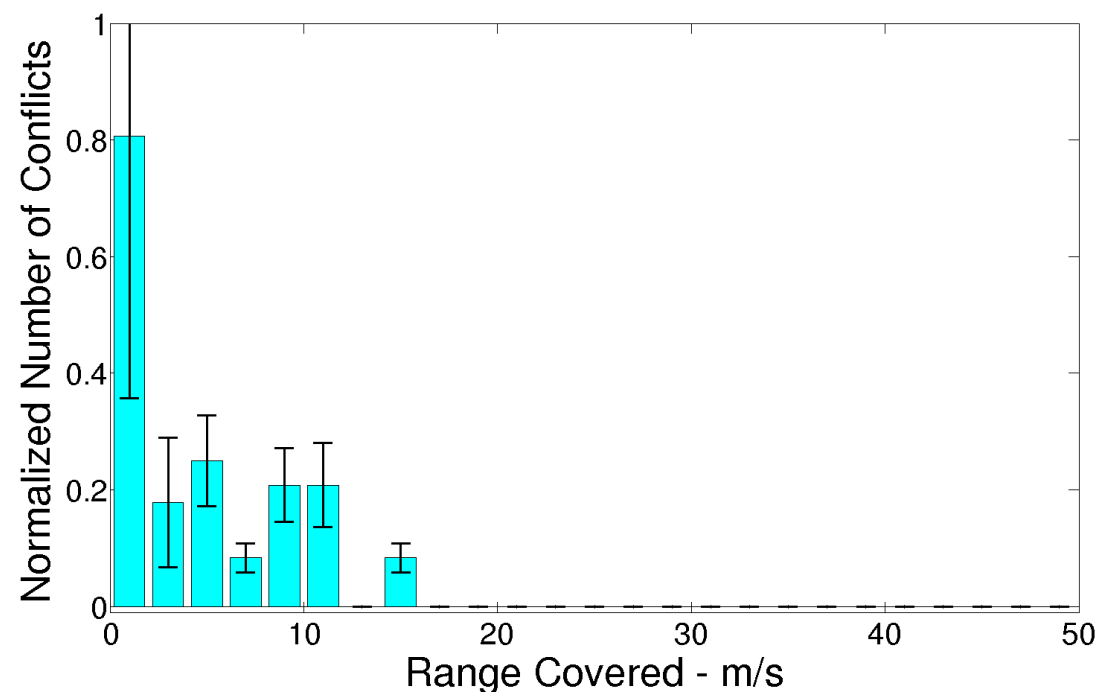

Figure 5.96: The $\Delta V$ distribution for seven influential neighbors and $\delta_{\text {attraction }}=1.5$ for the Flock barn swallow data set under the global coordinate system with the variable speed maneuver. 


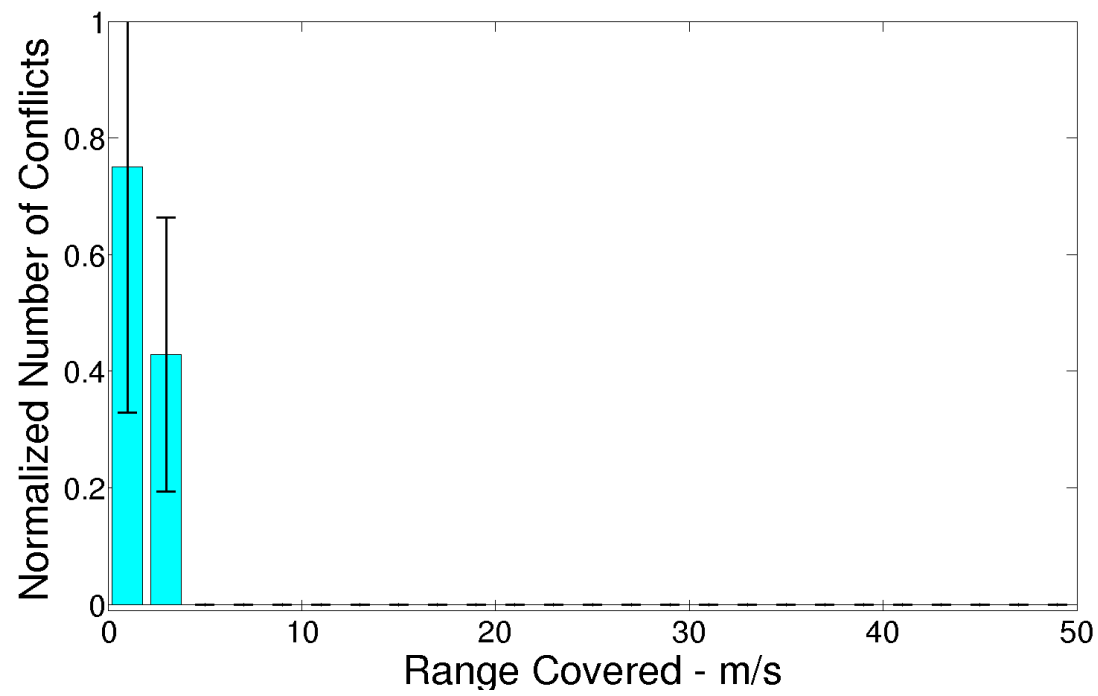

Figure 5.97: The $\Delta V$ distribution for three influential neighbors and $\delta_{\text {attraction }}=$ infinity for the Trial03 barn swallow data set under the global coordinate system with the variable speed maneuver. 


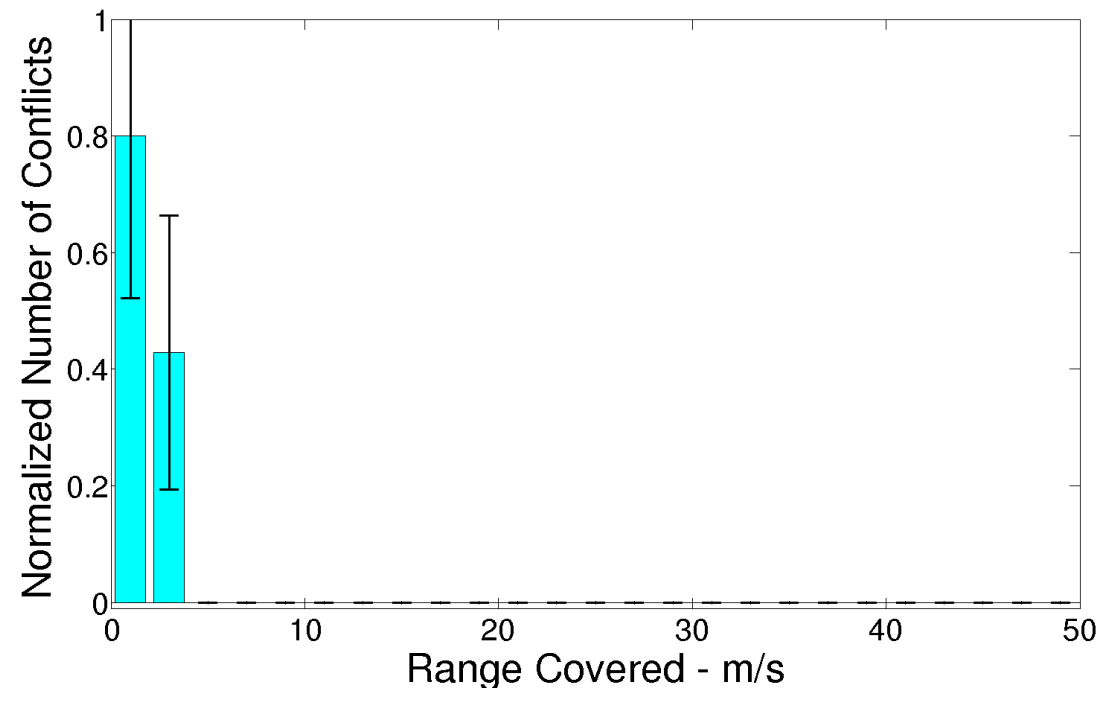

Figure 5.98: The $\Delta V$ distribution for seven influential neighbors and $\delta_{\text {attraction }}=$ infinity for the Trial03 barn swallow data set under the global coordinate system with the variable speed maneuver.

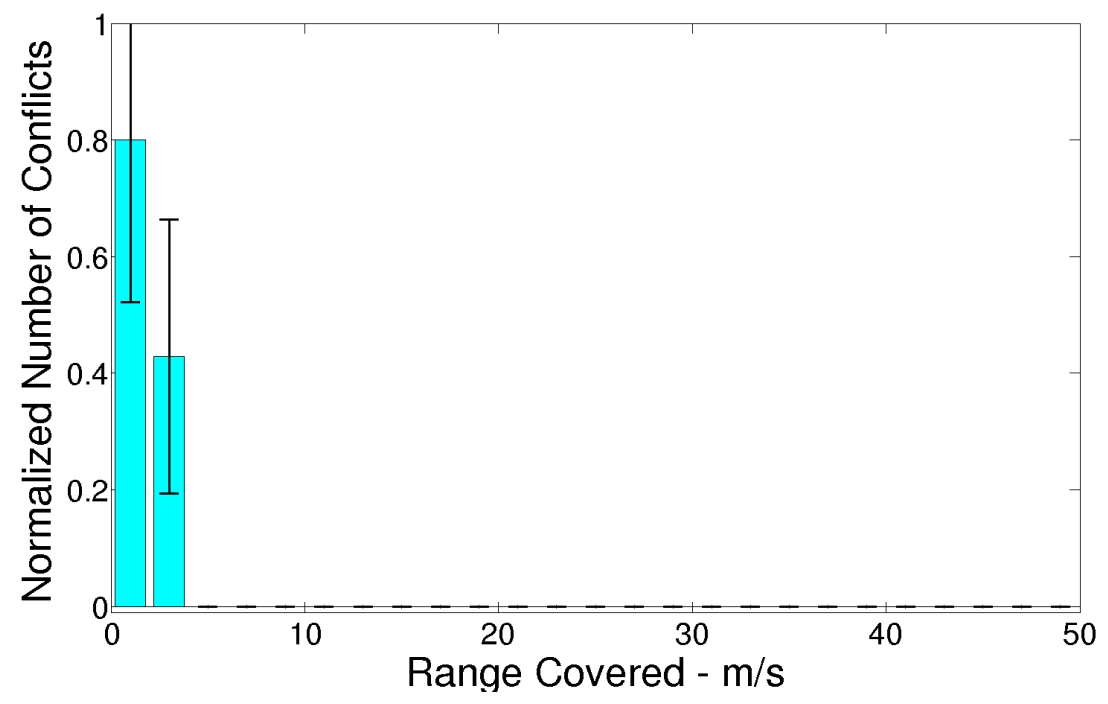

Figure 5.99: The $\Delta V$ distribution for the maximum number of influential neighbors and $\delta_{\text {attraction }}=$ infinity for the Trial03 barn swallow data set under the global coordinate system with the variable speed maneuver. 


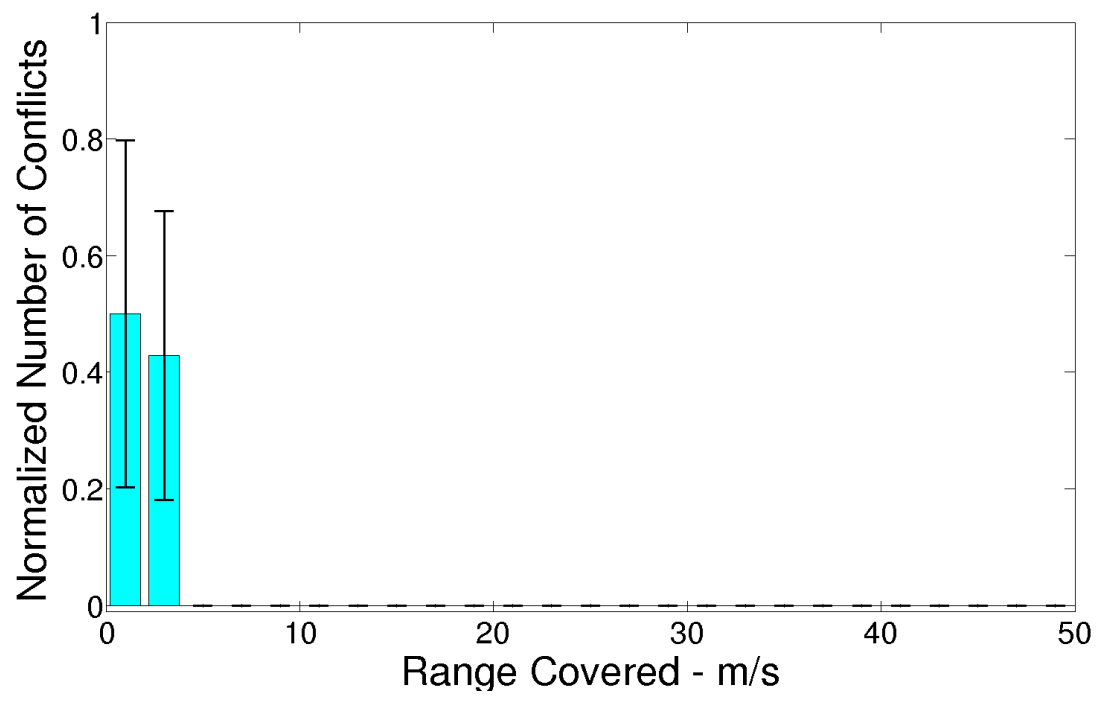

Figure 5.100: The $\Delta V$ distribution for seven influential neighbors and $\delta_{\text {attraction }}=5 \mathrm{~m}$ for the Trial03 barn swallow data set under the global coordinate system with the variable speed maneuver.

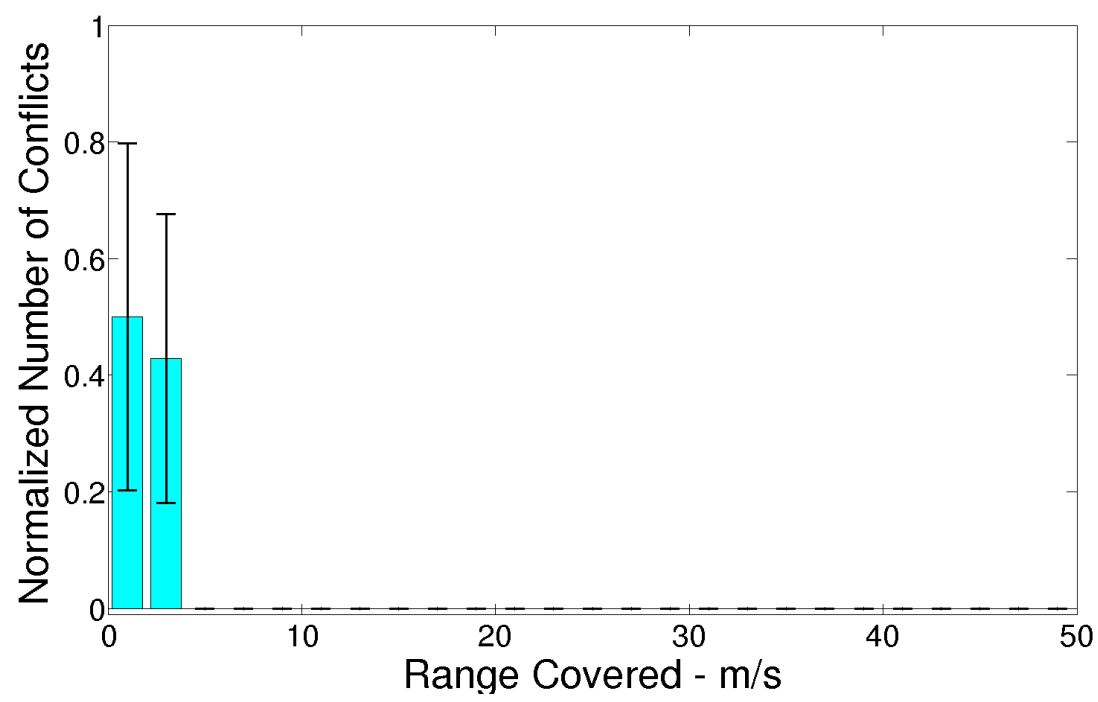

Figure 5.101: The $\Delta V$ distribution for seven influential neighbors and $\delta_{\text {attraction }}=3 \mathrm{~m}$ for the Trial03 barn swallow data set under the global coordinate system with the variable speed maneuver. 


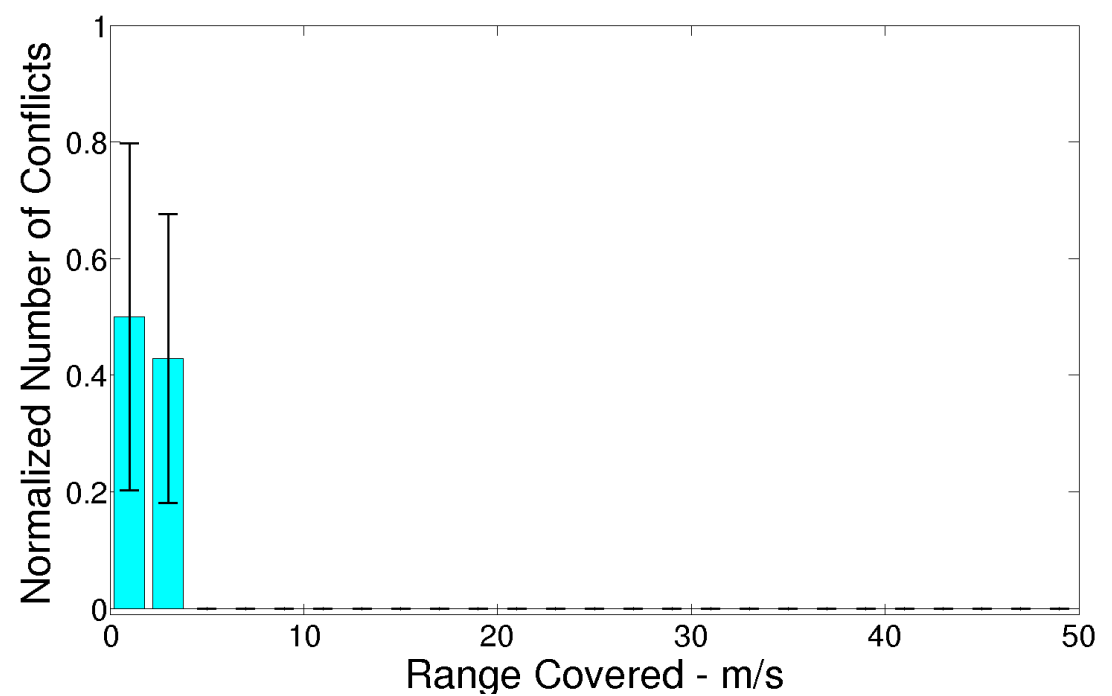

Figure 5.102: The $\Delta V$ distribution for seven influential neighbors and $\delta_{\text {attraction }}=1.5 \mathrm{~m}$ for the Trial03 barn swallow data set under the global coordinate system with the variable speed maneuver. 


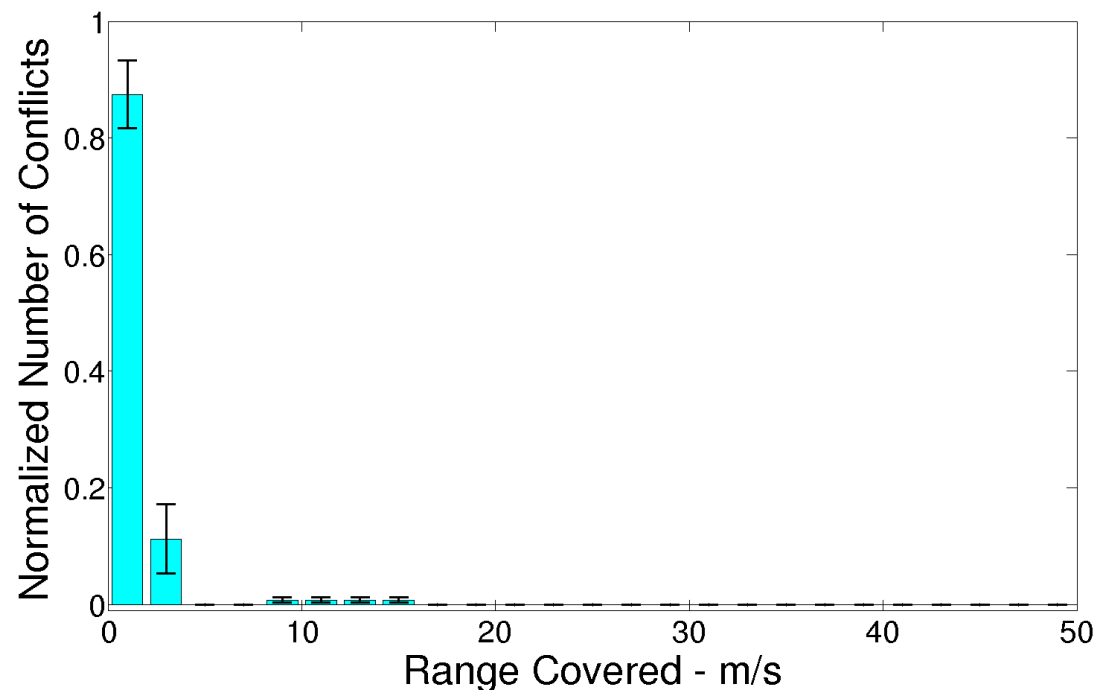

Figure 5.103: The $\Delta V$ distribution for three influential neighbors and $\delta_{\text {attraction }}=$ infinity for the Trial04 barn swallow data set under the global coordinate system with the variable speed maneuver. 


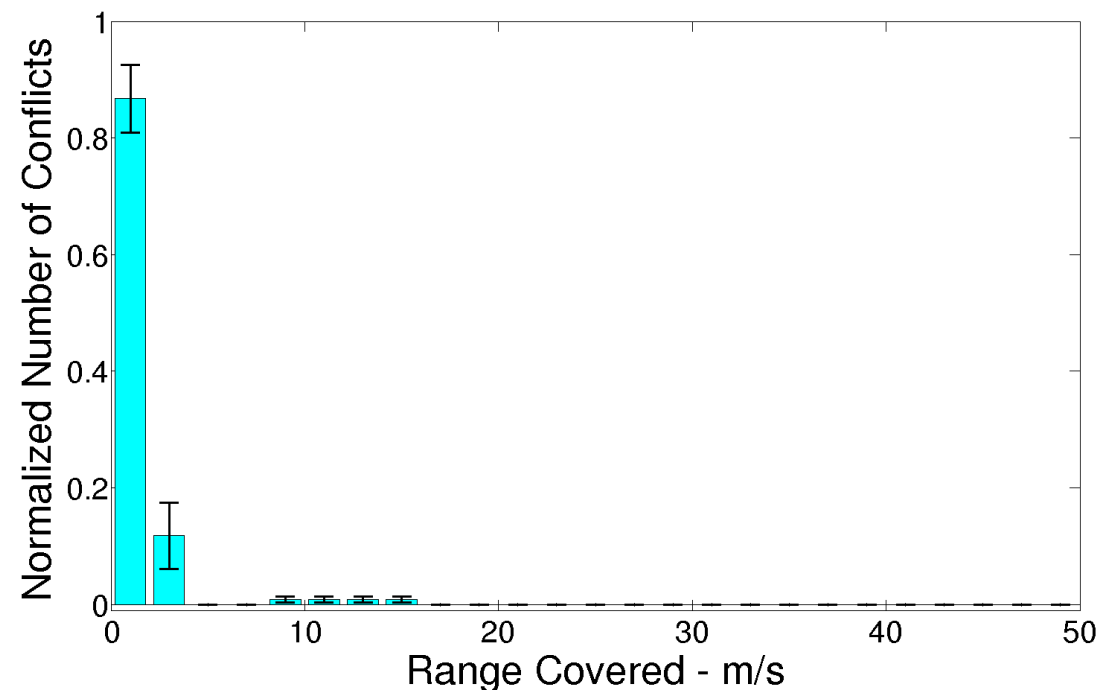

Figure 5.104: The $\Delta V$ distribution for three influential neighbors and $\delta_{\text {attraction }}=5 \mathrm{~m}$ for the Trial04 barn swallow data set under the global coordinate system with the variable speed maneuver.

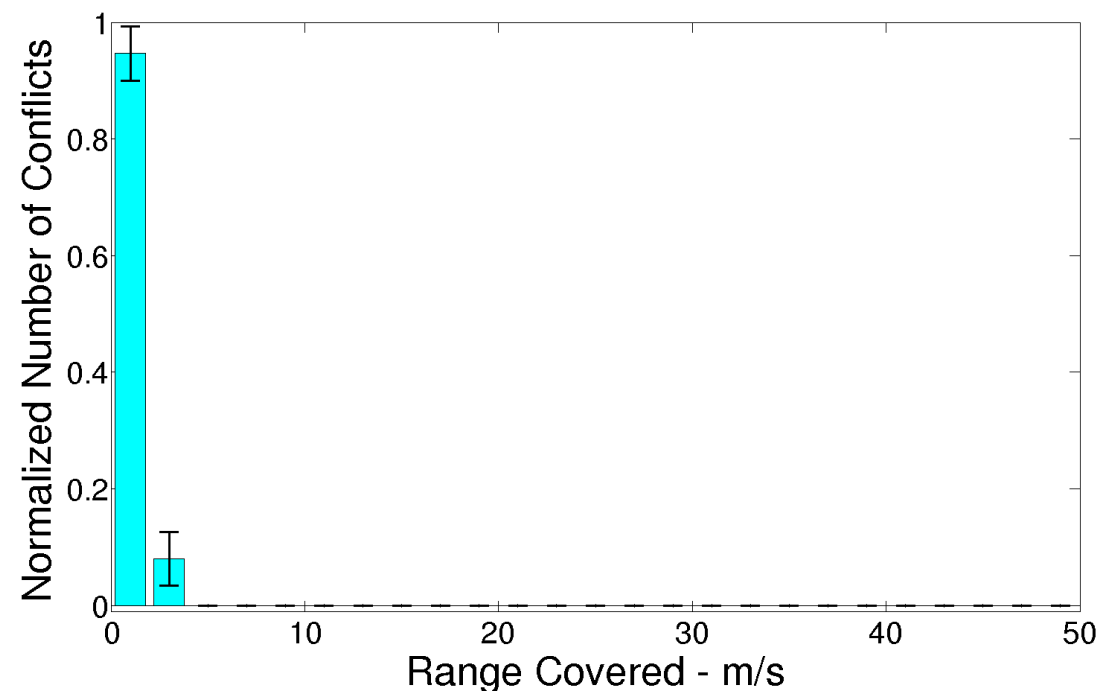

Figure 5.105: The $\Delta V$ distribution for three influential neighbors and $\delta_{\text {attraction }}=3 \mathrm{~m}$ for the Trial04 barn swallow data set under the global coordinate system with the variable speed maneuver. 


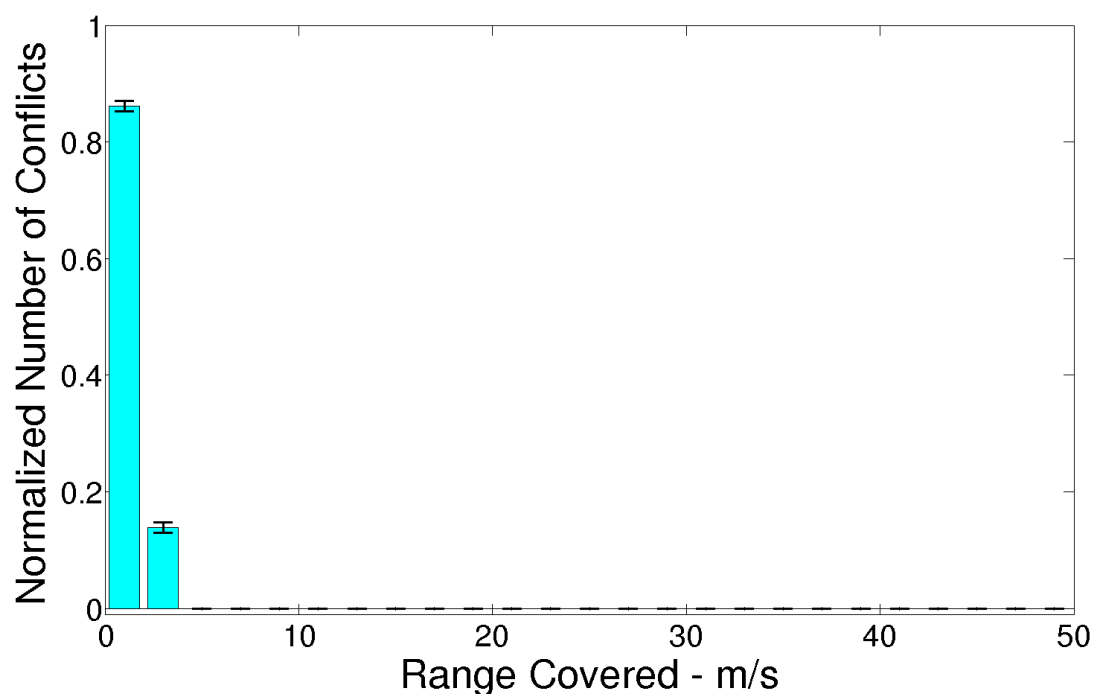

Figure 5.106: The $\Delta V$ distribution for three influential neighbors and $\delta_{\text {attraction }}=1.5 \mathrm{~m}$ for the Trial04 barn swallow data set under the global coordinate system with the variable speed maneuver. 


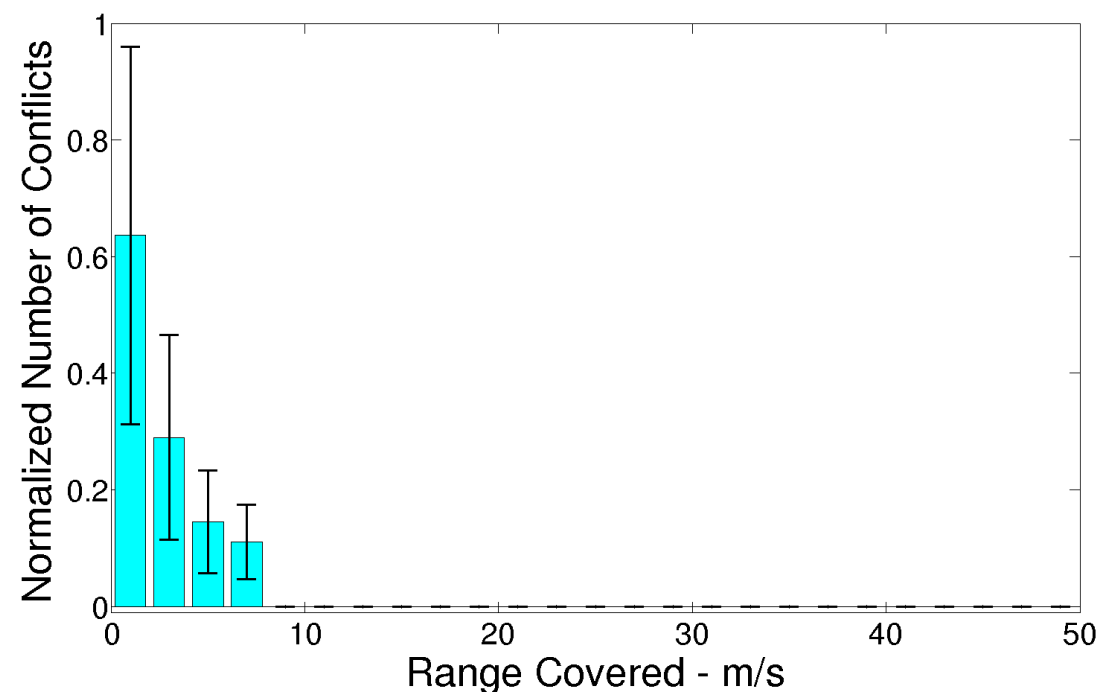

Figure 5.107: The $\Delta V$ distribution for three influential neighbors and $\delta_{\text {attraction }}=$ infinity for the Trial02-2012 barn swallow data set under the global coordinate system with the variable speed maneuver. 


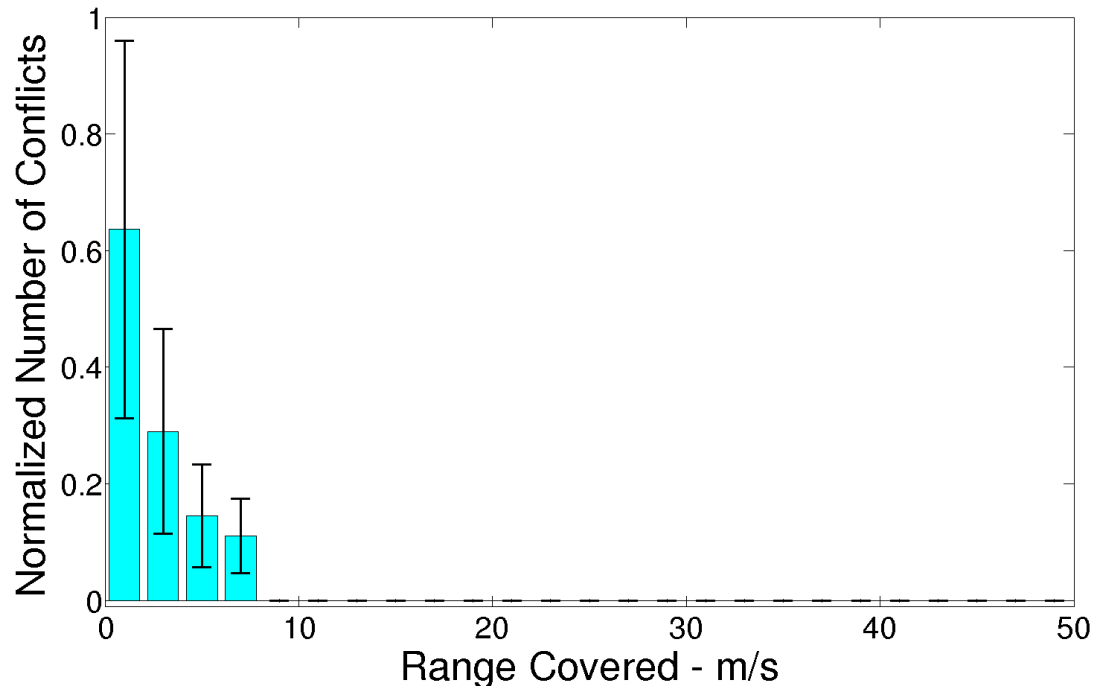

Figure 5.108: The $\Delta V$ distribution for three influential neighbors and $\delta_{\text {attraction }}=5 \mathrm{~m}$ for the Trial02-2012 barn swallow data set under the global coordinate system with the variable speed maneuver.

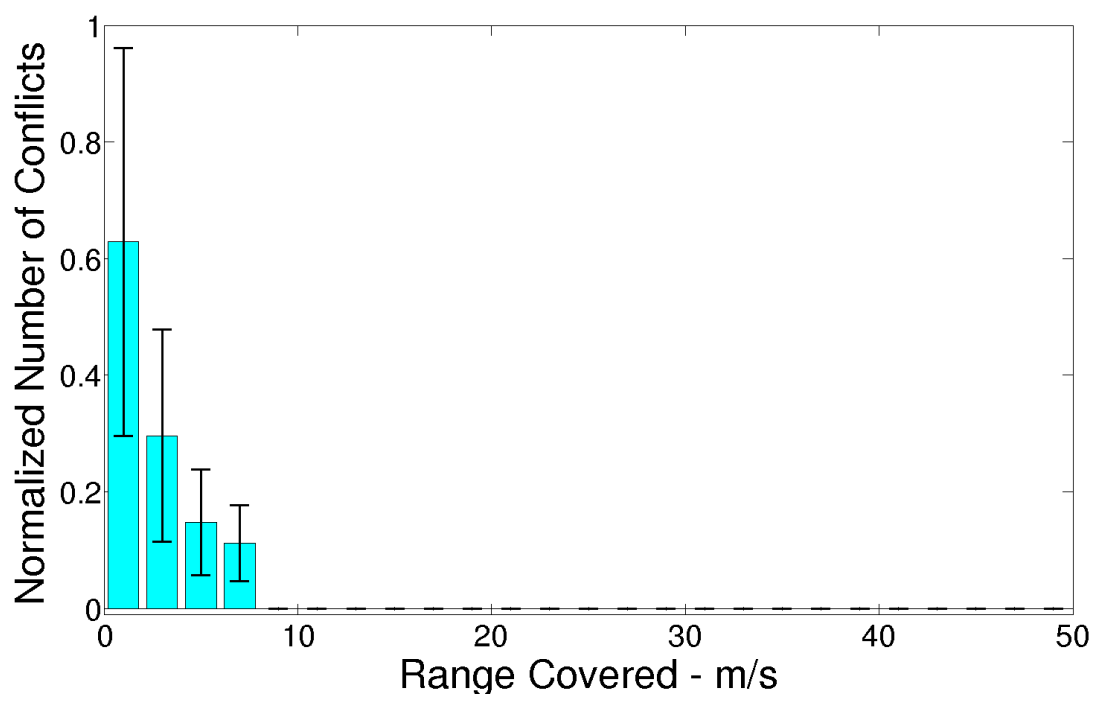

Figure 5.109: The $\Delta V$ distribution for three influential neighbors and $\delta_{\text {attraction }}=3 \mathrm{~m}$ for the Trial02-2012 barn swallow data set under the global coordinate system with the variable speed maneuver. 


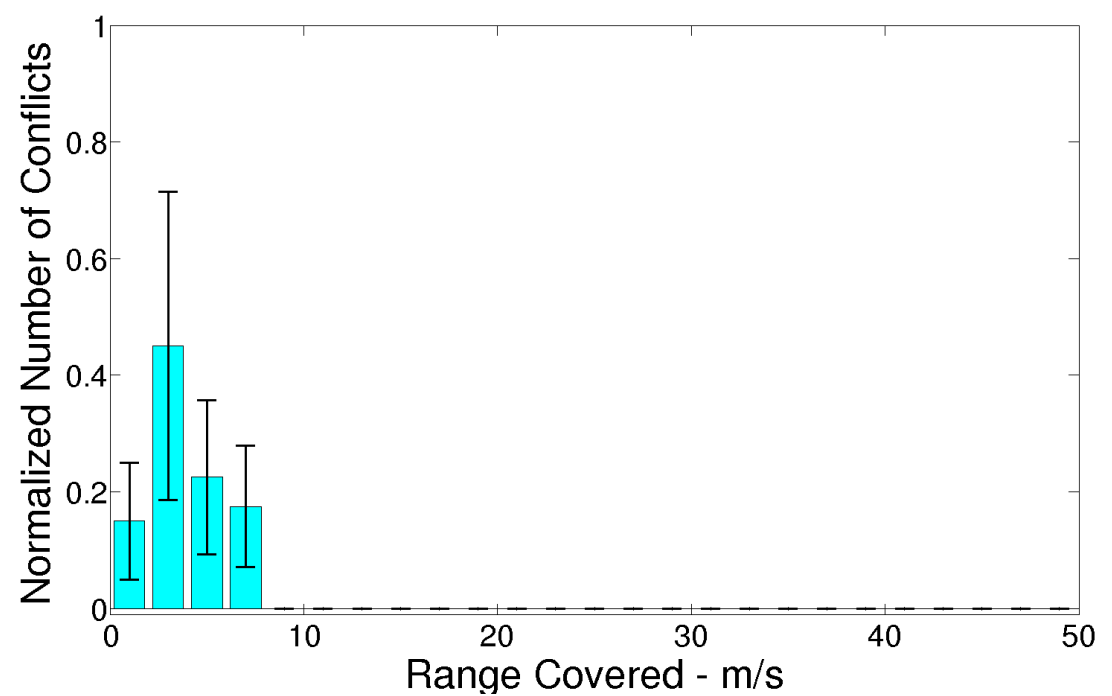

Figure 5.110: The $\Delta V$ distribution for three influential neighbors and $\delta_{\text {attraction }}=1.5 \mathrm{~m}$ for the Trial02-2012 barn swallow data set under the global coordinate system with the variable speed maneuver. 


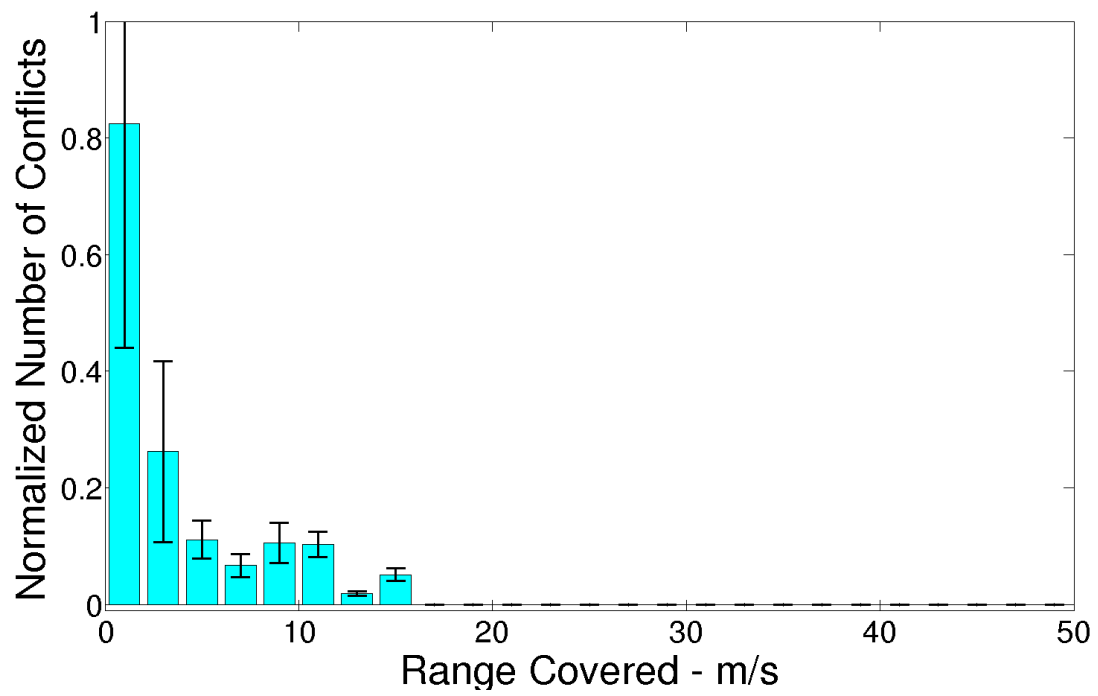

Figure 5.111: The $\Delta V$ distribution for three influential neighbors and $\delta_{\text {attraction }}=$ infinity for the Flock barn swallow data set under the body-fixed coordinate system with the constant speed maneuver. 


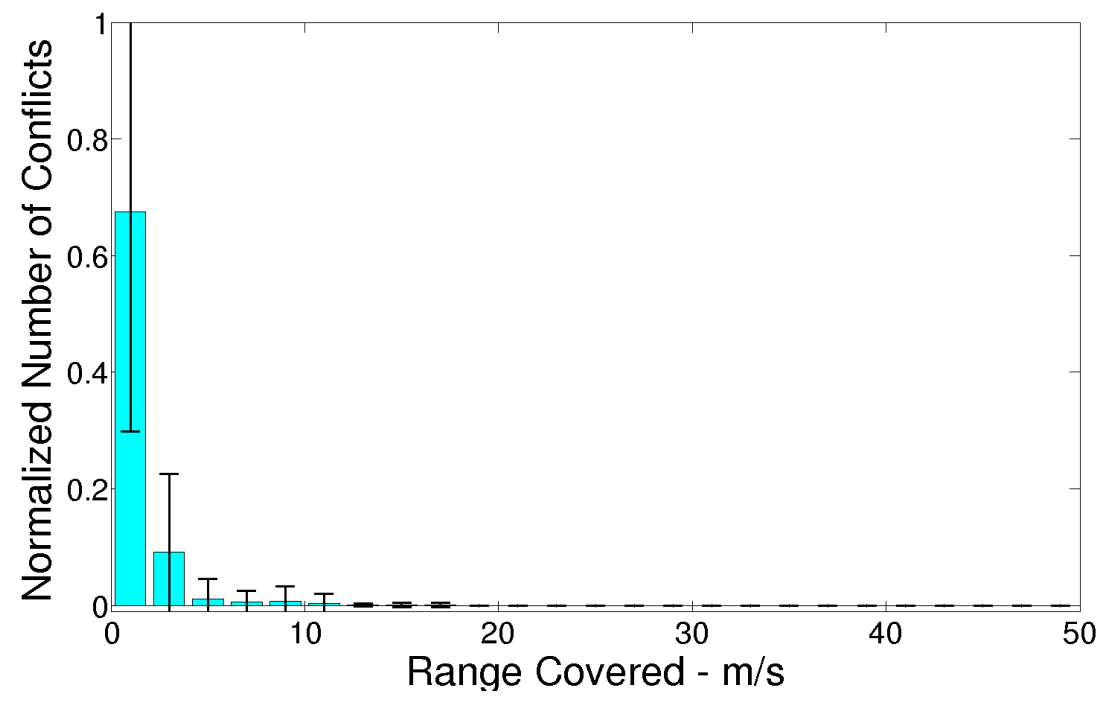

Figure 5.112: The $\Delta V$ distribution for seven influential neighbors and $\delta_{\text {attraction }}=$ infinity for the Flock barn swallow data set under the body-fixed coordinate system with the constant speed maneuver.

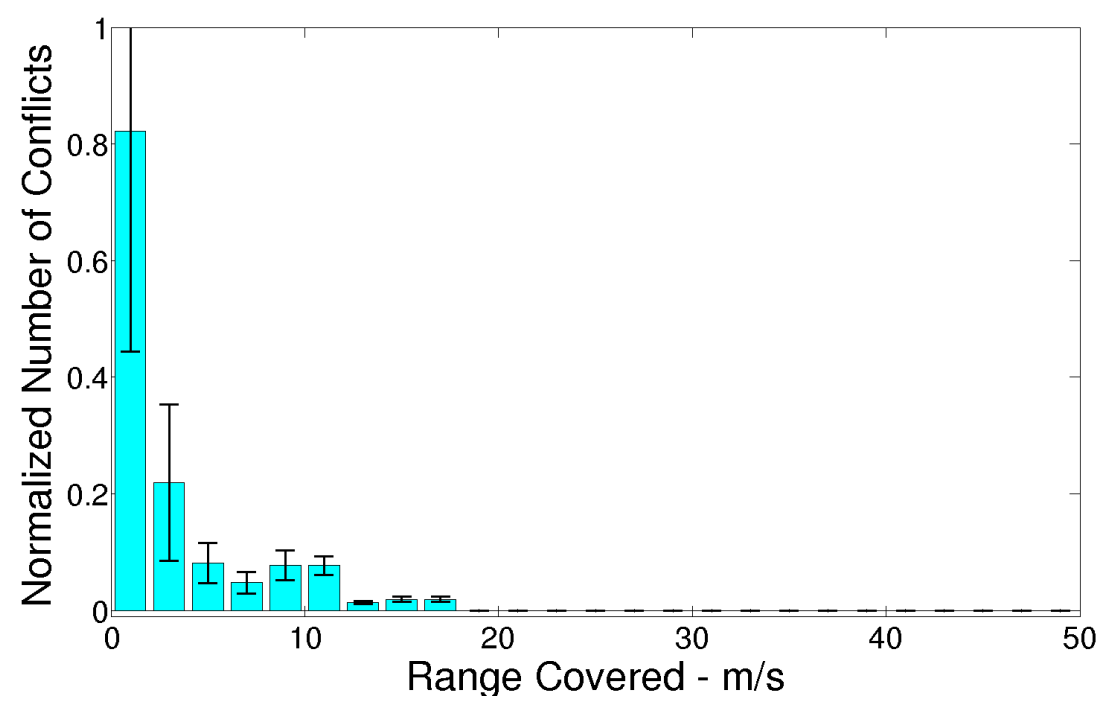

Figure 5.113: The $\Delta V$ distribution for the maximum number of influential neighbors and $\delta_{\text {attraction }}=$ infinity for the Flock barn swallow data set under the body-fixed coordinate system with the constant speed maneuver. 


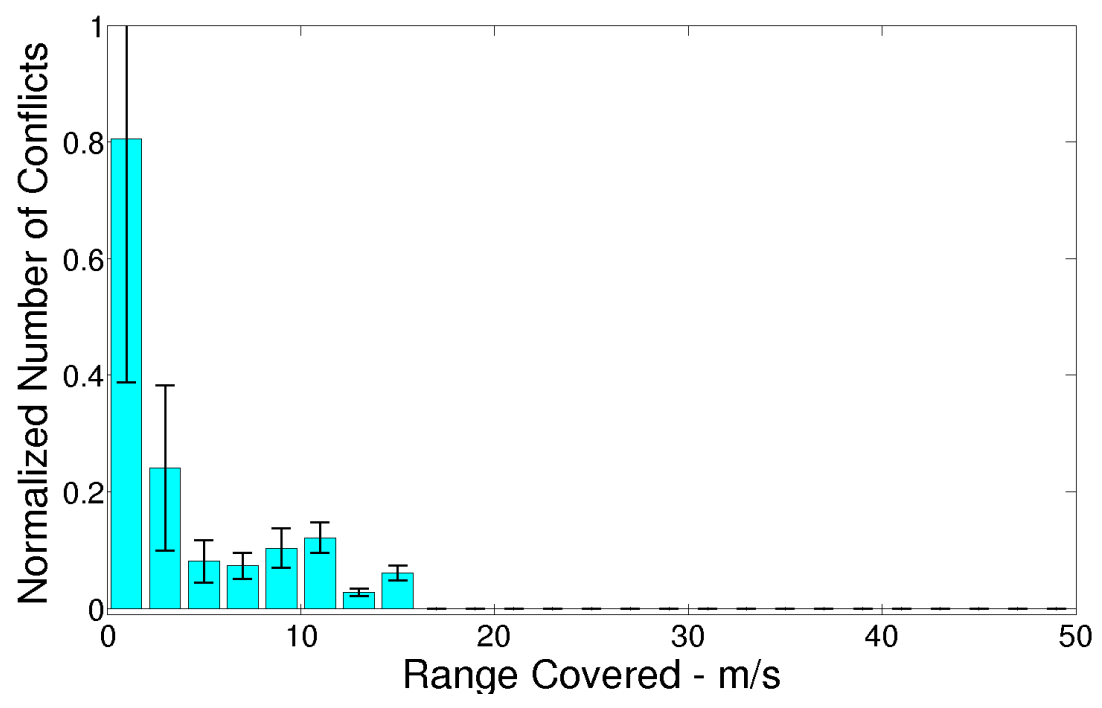

Figure 5.114: The $\Delta V$ distribution for seven influential neighbors and $\delta_{\text {attraction }}=5 \mathrm{~m}$ for the Flock barn swallow data set under the body-fixed coordinate system with the constant speed maneuver.

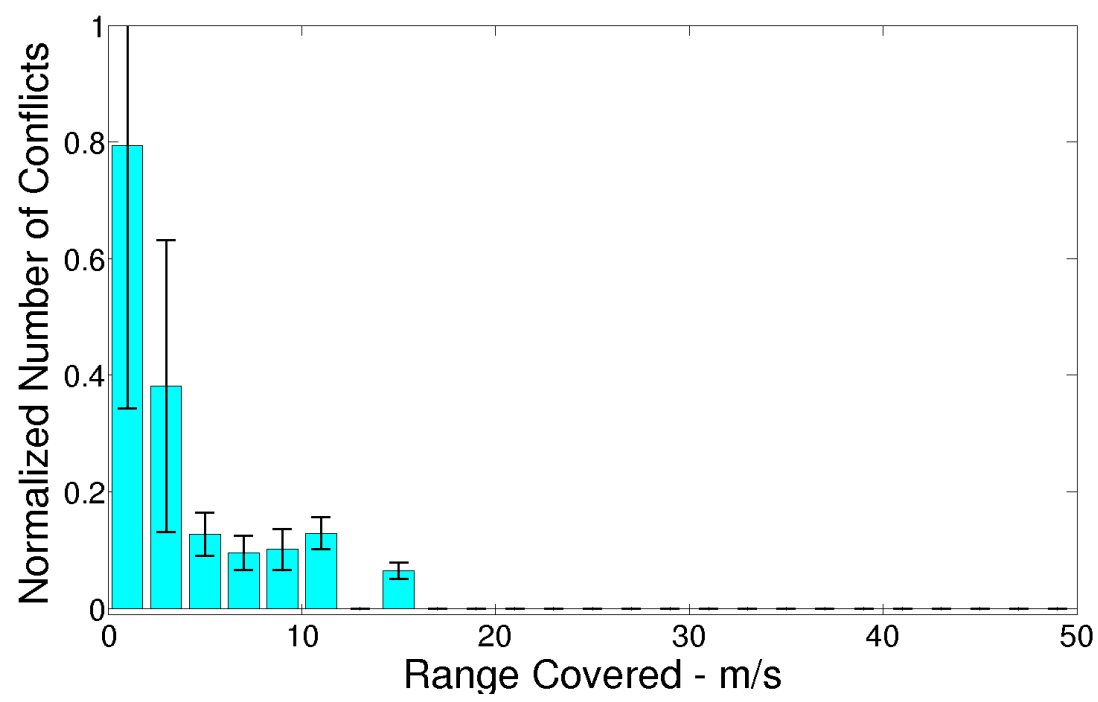

Figure 5.115: The $\Delta V$ distribution for seven influential neighbors and $\delta_{\text {attraction }}=3 \mathrm{~m}$ for the Flock barn swallow data set under the body-fixed coordinate system with the constant speed maneuver. 


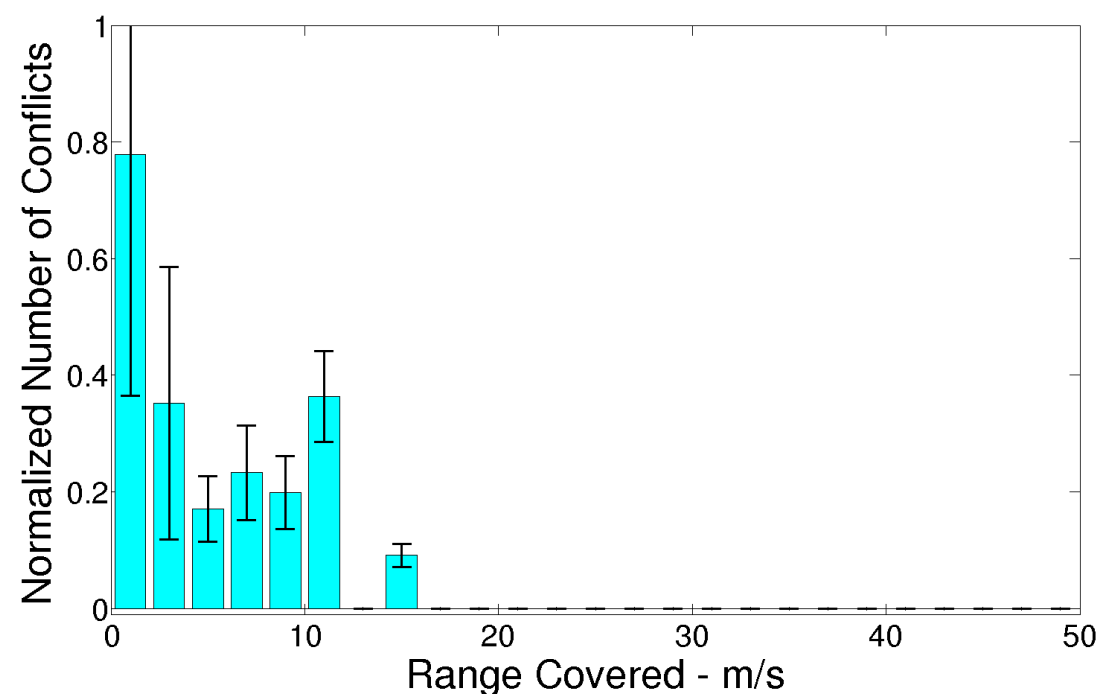

Figure 5.116: The $\Delta V$ distribution for seven influential neighbors and $\delta_{\text {attraction }}=1.5 \mathrm{~m}$ for the Flock barn swallow data set under the body-fixed coordinate system with the constant speed maneuver. 


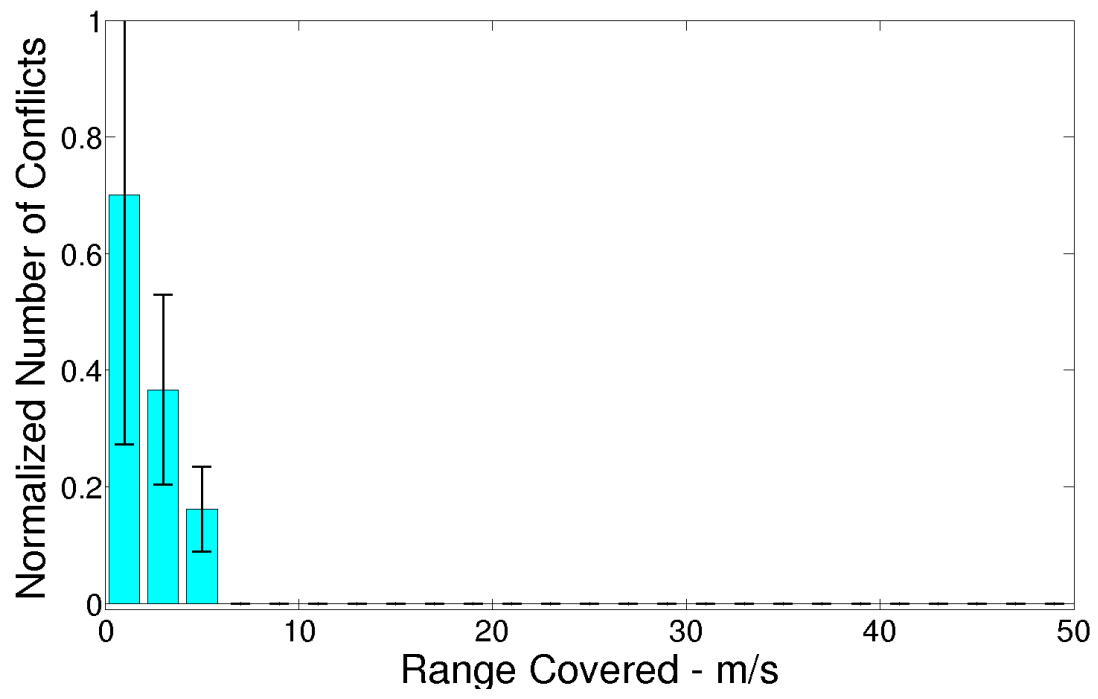

Figure 5.117: The $\Delta V$ distribution for three influential neighbors and $\delta_{\text {attraction }}=$ infinity for the Trial03 barn swallow data set under the body-fixed coordinate system with the constant speed maneuver. 


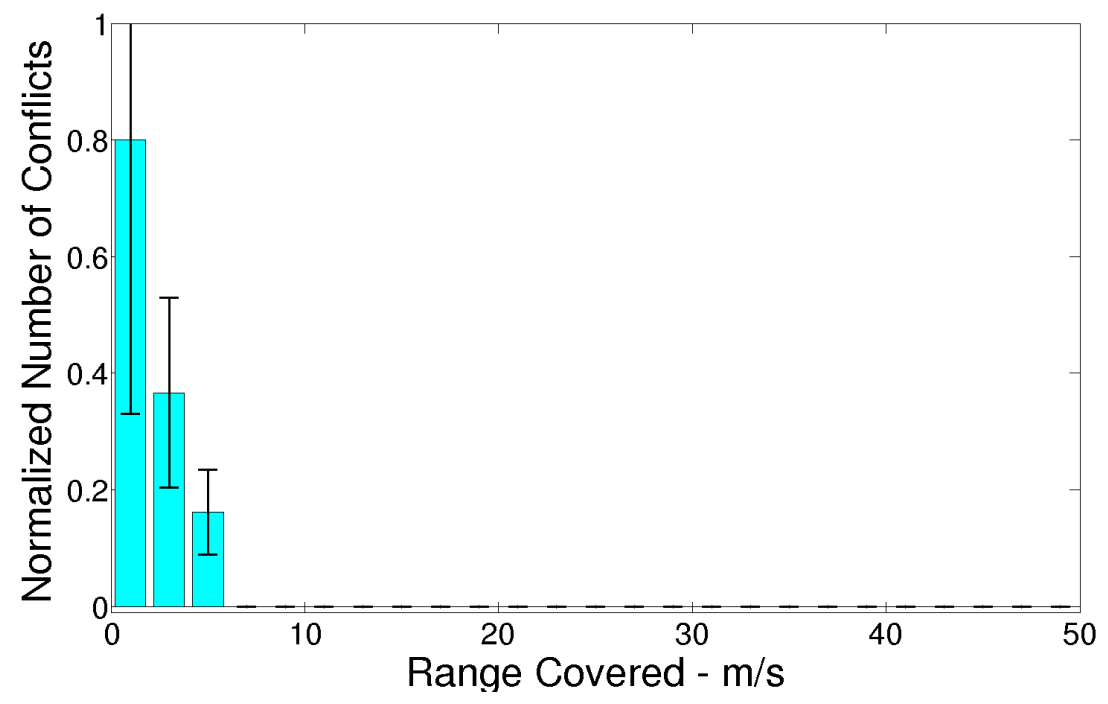

Figure 5.118: The $\Delta V$ distribution for seven influential neighbors and $\delta_{\text {attraction }}=$ infinity for the Trial03 barn swallow data set under the body-fixed coordinate system with the constant speed maneuver.

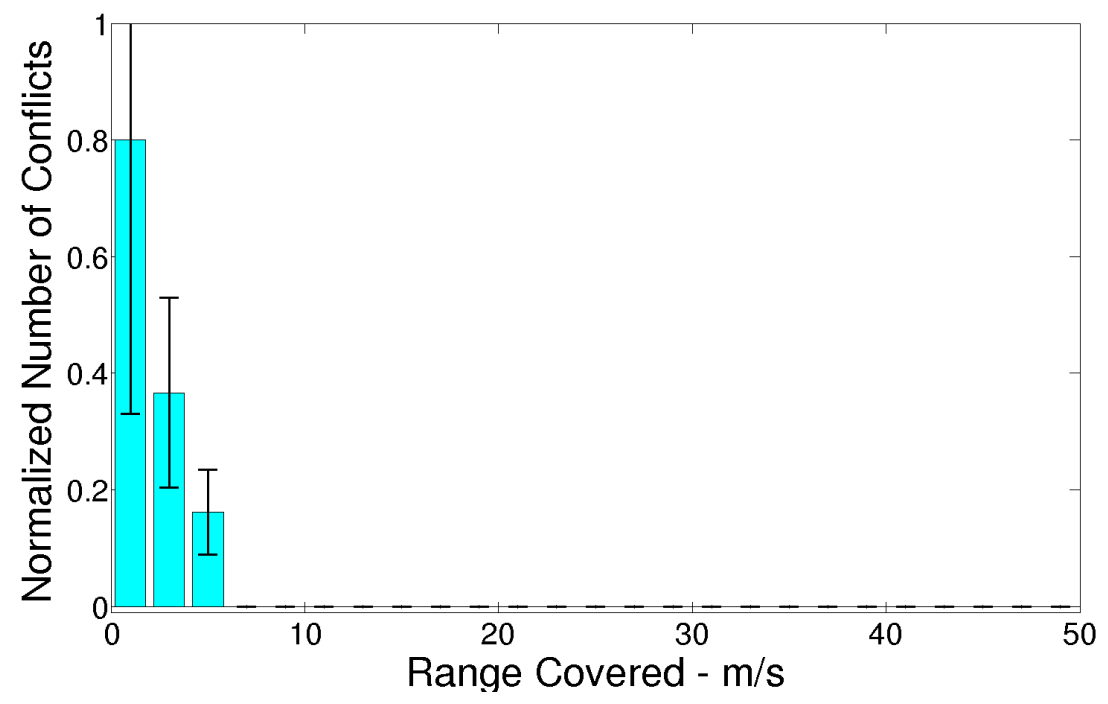

Figure 5.119: The $\Delta V$ distribution for the maximum number of influential neighbors and $\delta_{\text {attraction }}=$ infinity for the Trial03 barn swallow data set under the body-fixed coordinate system with the constant speed maneuver. 


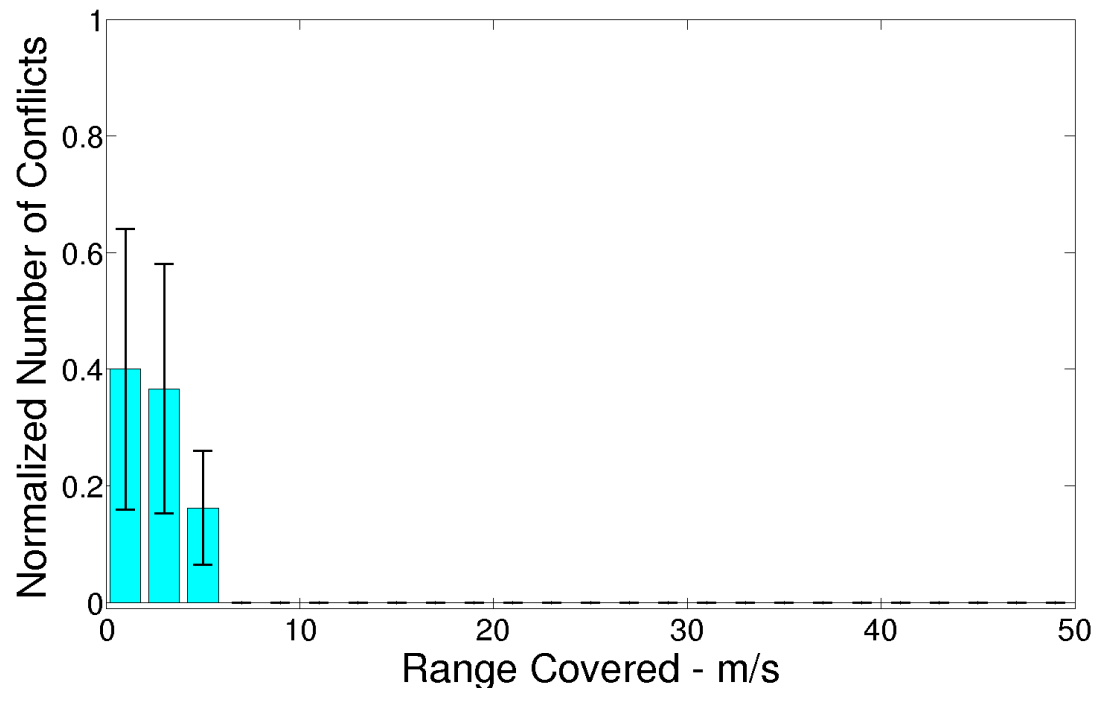

Figure 5.120: The $\Delta V$ distribution for seven influential neighbors and $\delta_{\text {attraction }}=5 \mathrm{~m}$ for the Trial03 barn swallow data set under the body-fixed coordinate system with the constant speed maneuver.

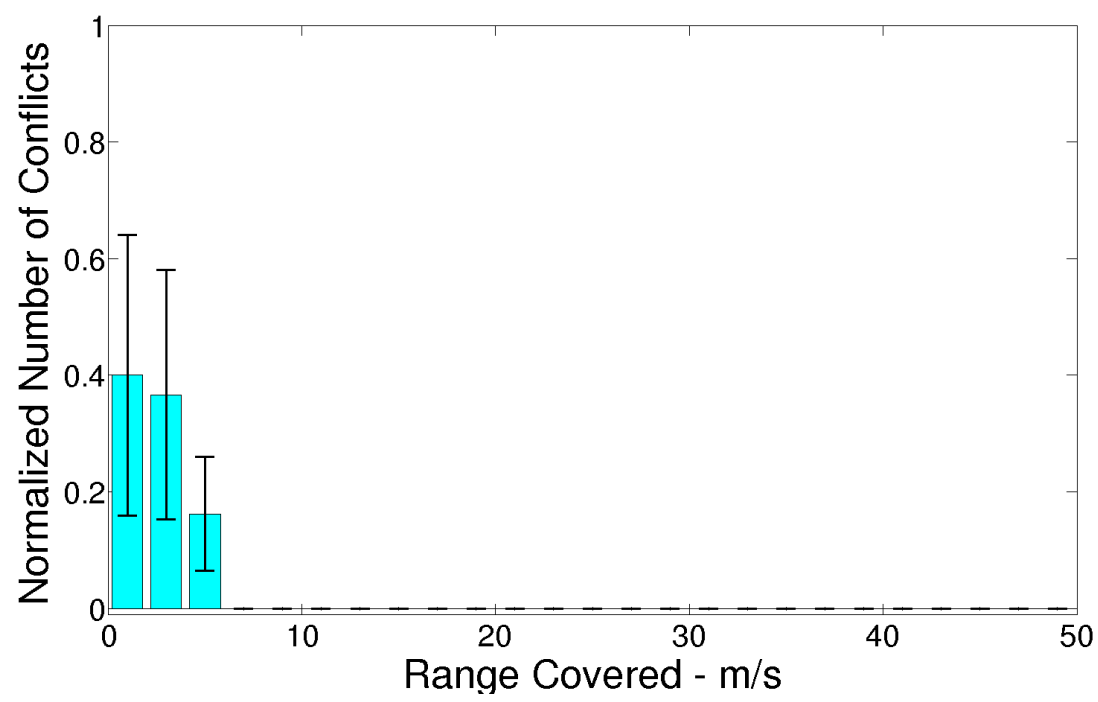

Figure 5.121: The $\Delta V$ distribution for seven influential neighbors and $\delta_{\text {attraction }}=3 \mathrm{~m}$ for the Trial03 barn swallow data set under the body-fixed coordinate system with the constant speed maneuver. 


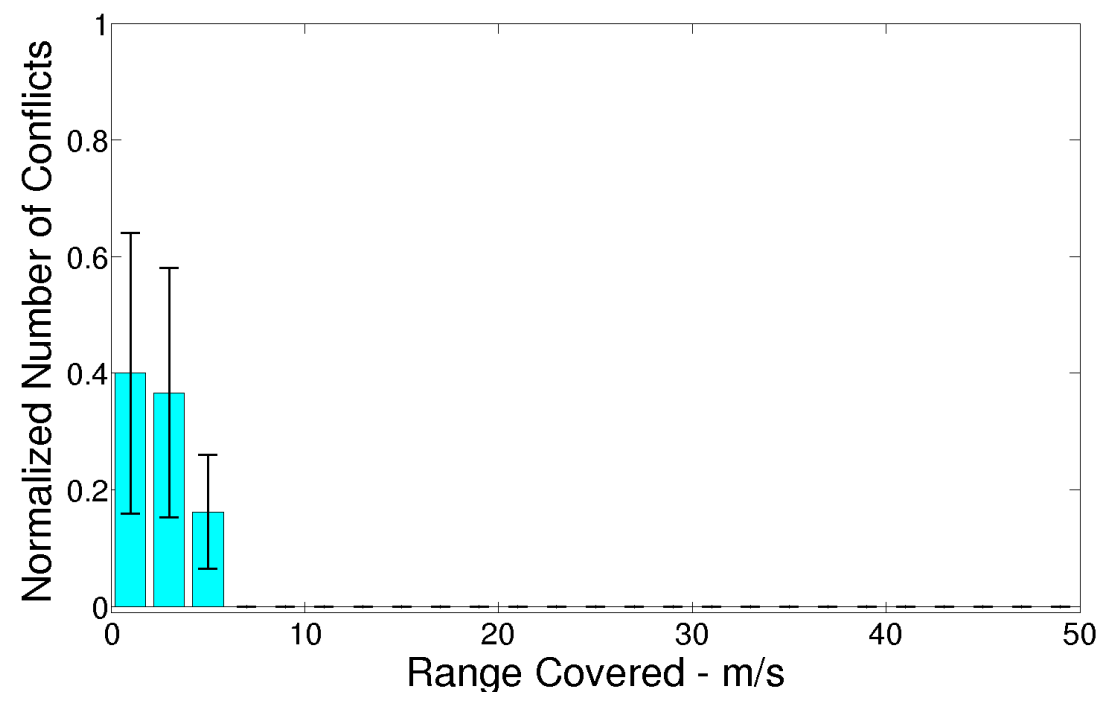

Figure 5.122: The $\Delta V$ distribution for seven influential neighbors and $\delta_{\text {attraction }}=1.5 \mathrm{~m}$ for the Trial03 barn swallow data set under the body-fixed coordinate system with the constant speed maneuver.

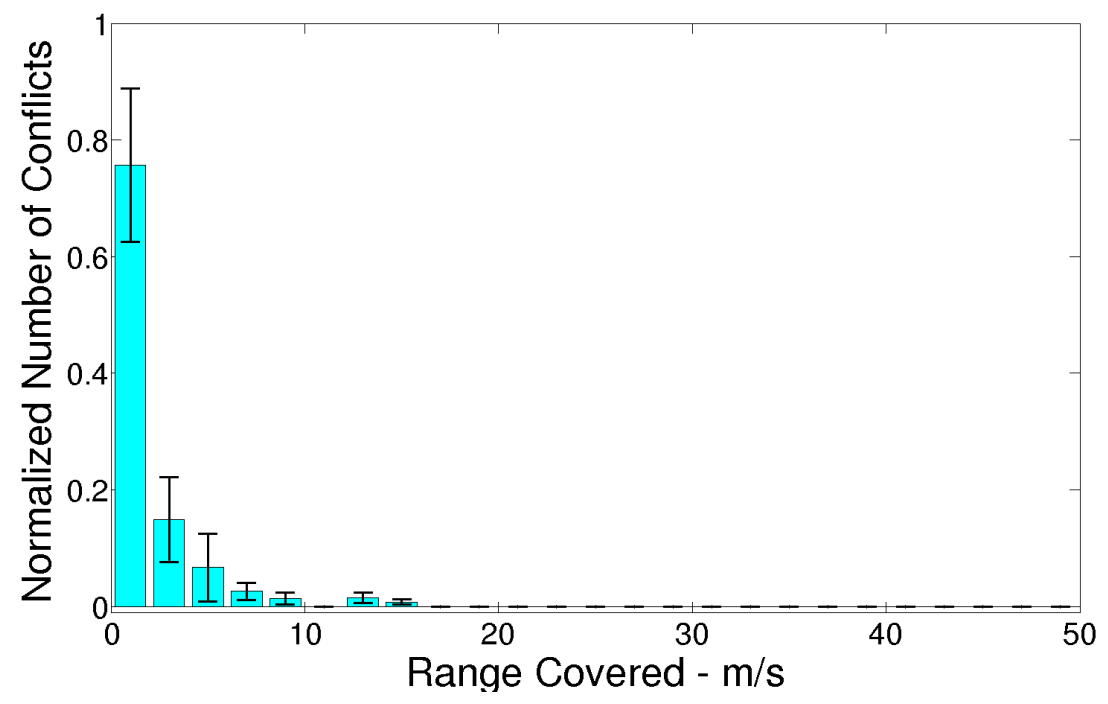

Figure 5.123: The $\Delta V$ distribution for three influential neighbors and $\delta_{\text {attraction }}=$ infinity for the Trial04 barn swallow data set under the body-fixed coordinate system with the constant speed maneuver. 


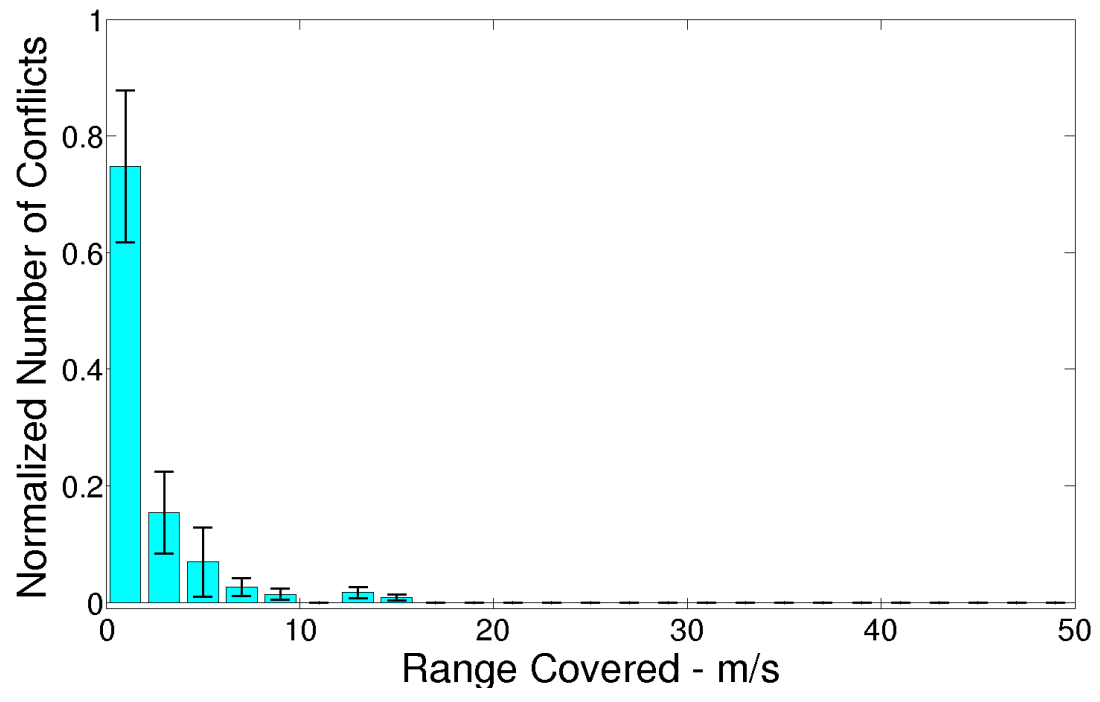

Figure 5.124: The $\Delta V$ distribution for three influential neighbors and $\delta_{\text {attraction }}=5 \mathrm{~m}$ for the Trial04 barn swallow data set under the body-fixed coordinate system with the constant speed maneuver.

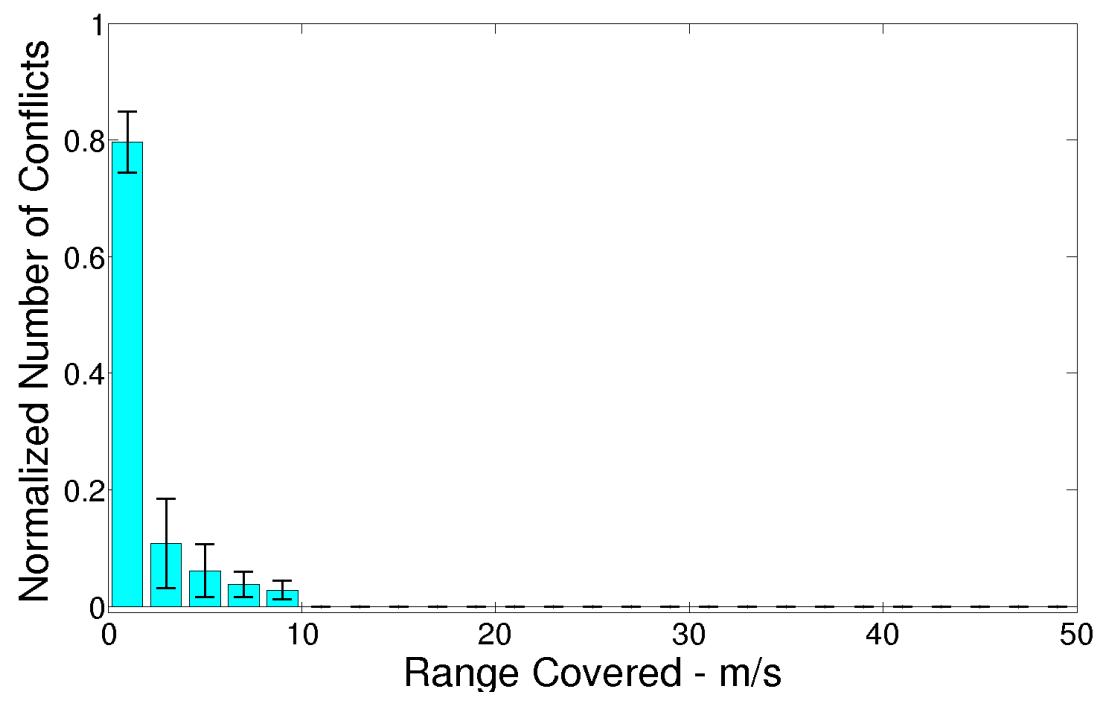

Figure 5.125: The $\Delta V$ distribution for three influential neighbors and $\delta_{\text {attraction }}=3 \mathrm{~m}$ for the Trial04 barn swallow data set under the body-fixed coordinate system with the constant speed maneuver. 


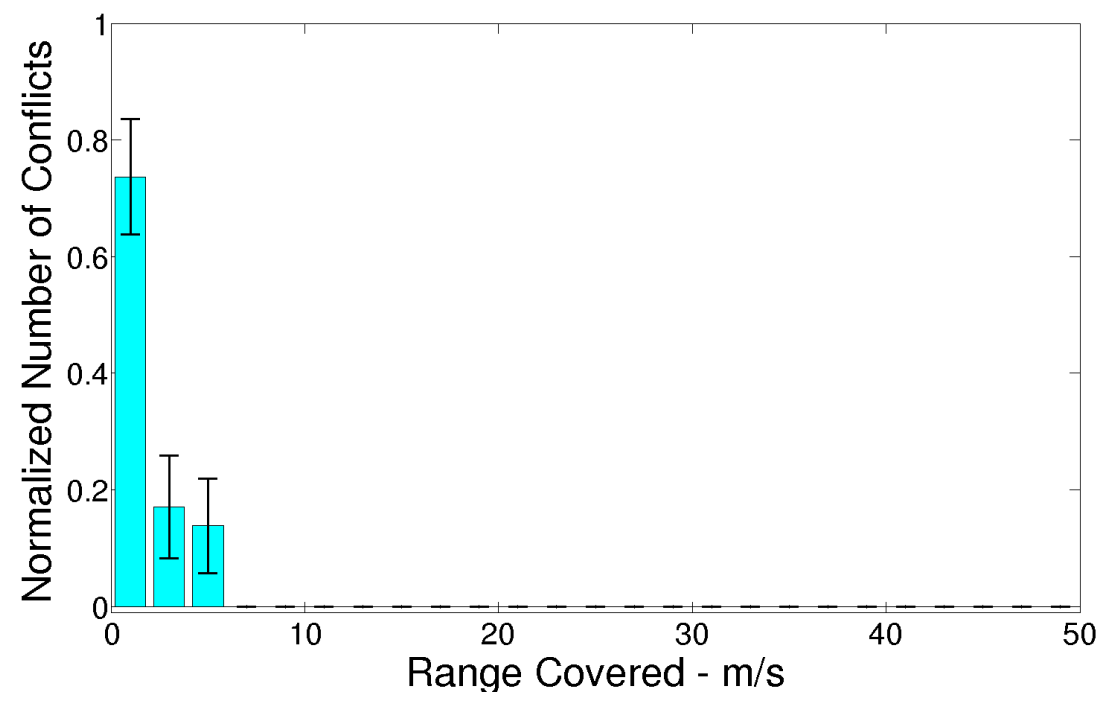

Figure 5.126: The $\Delta V$ distribution for three influential neighbors and $\delta_{\text {attraction }}=1.5 \mathrm{~m}$ for the Trial04 barn swallow data set under the body-fixed coordinate system with the constant speed maneuver.

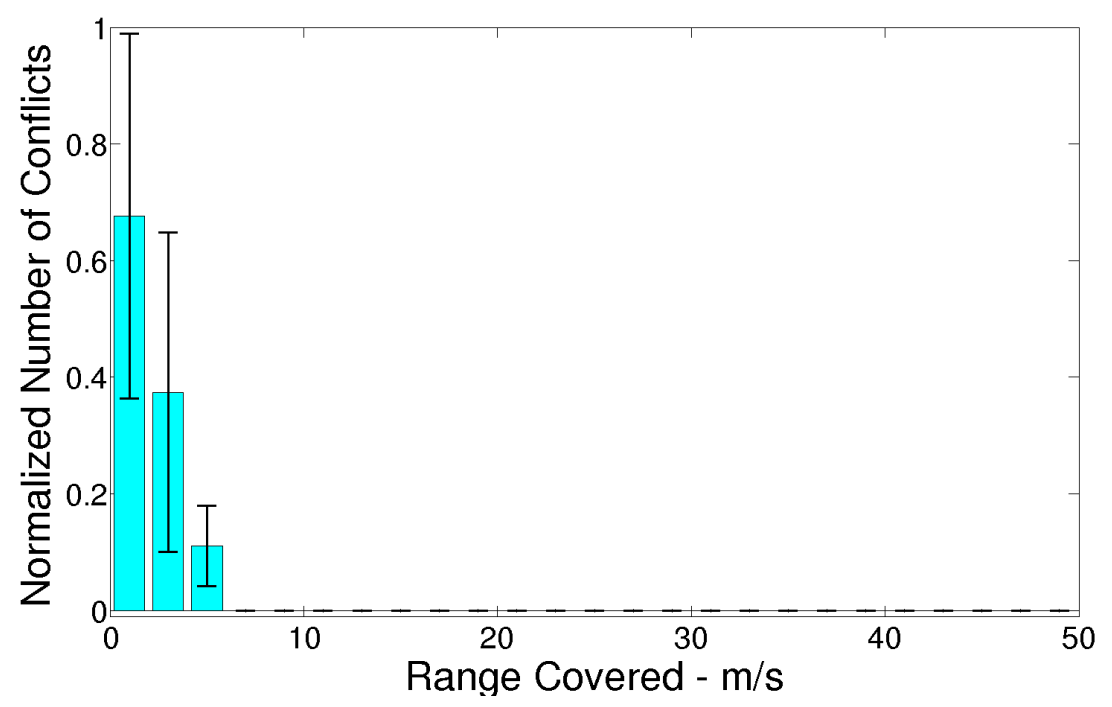

Figure 5.127: The $\Delta V$ distribution for three influential neighbors and $\delta_{\text {attraction }}=$ infinity for the Trial02-2012 barn swallow data set under the body-fixed coordinate system with the constant speed maneuver. 


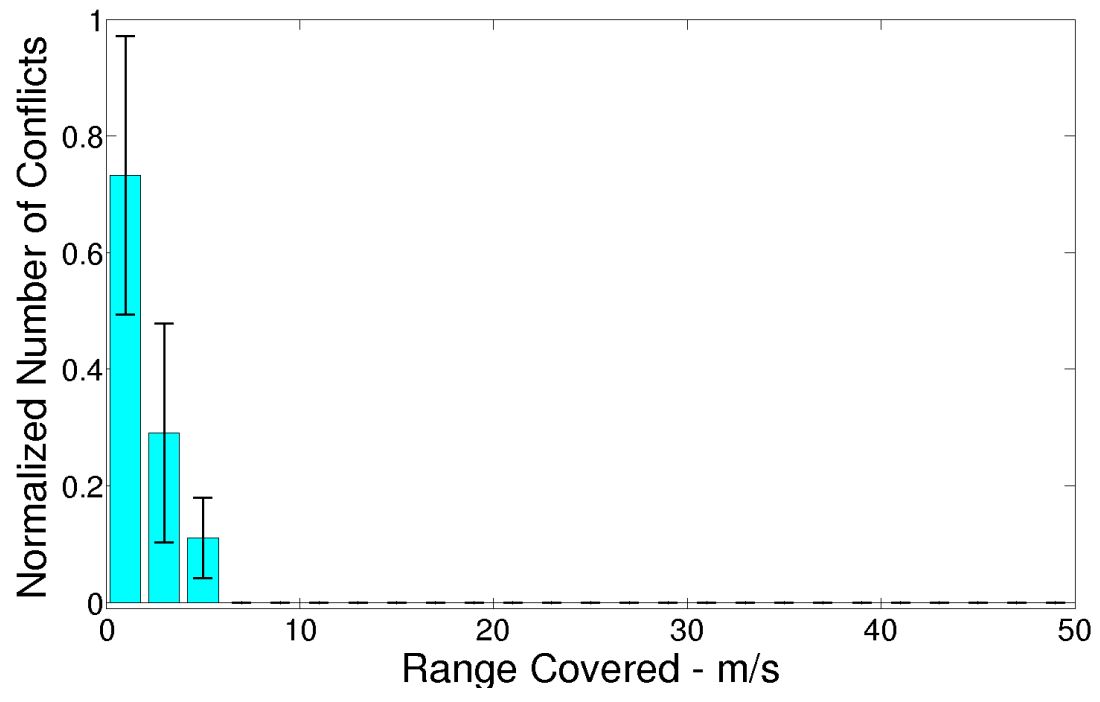

Figure 5.128: The $\Delta V$ distribution for three influential neighbors and $\delta_{\text {attraction }}=5 \mathrm{~m}$ for the Trial02-2012 barn swallow data set under the body-fixed coordinate system with the constant speed maneuver.

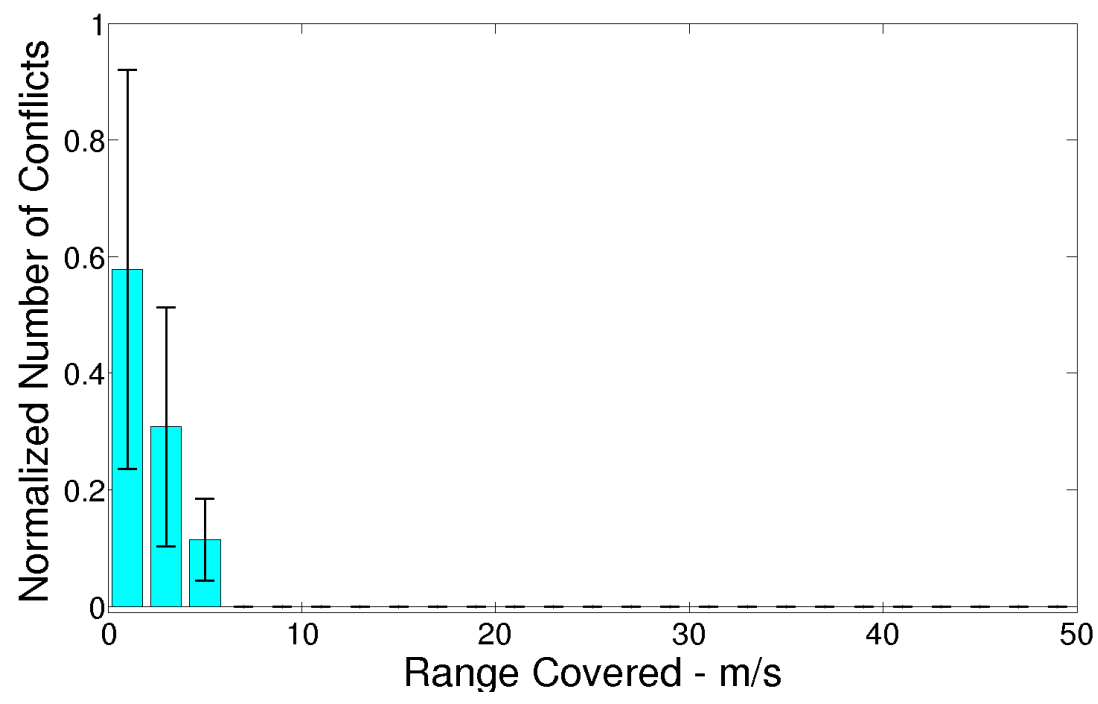

Figure 5.129: The $\Delta V$ distribution for three influential neighbors and $\delta_{\text {attraction }}=3 \mathrm{~m}$ for the Trial02-2012 barn swallow data set under the body-fixed coordinate system with the constant speed maneuver. 


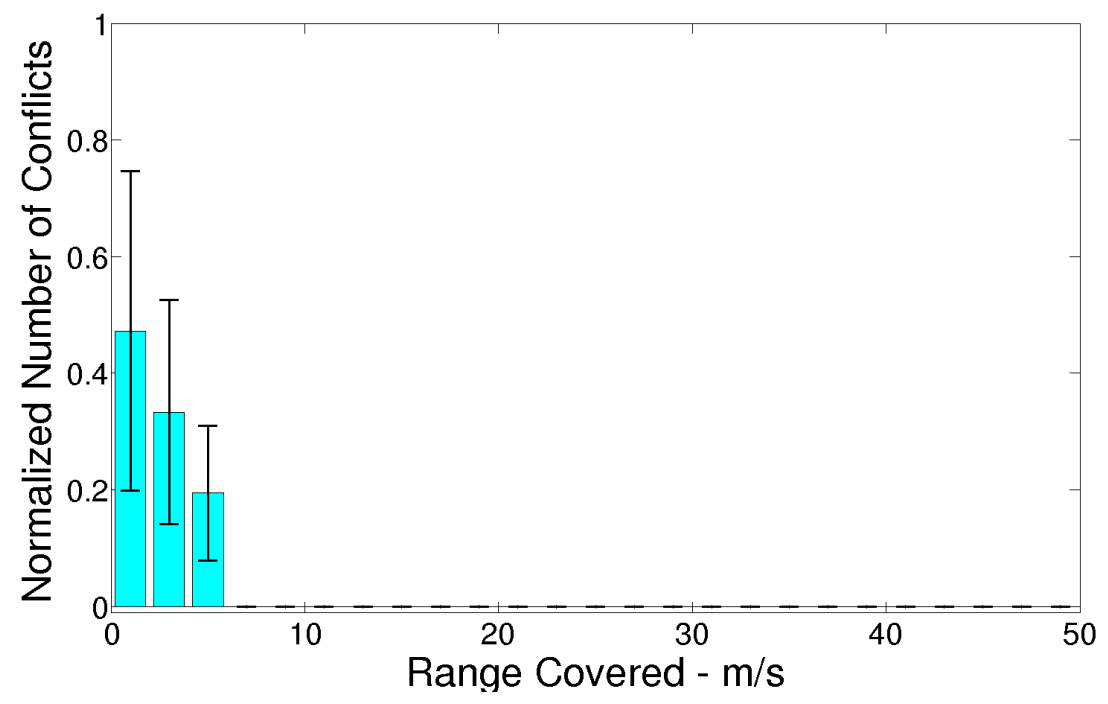

Figure 5.130: The $\Delta V$ distribution for three influential neighbors and $\delta_{\text {attraction }}=1.5 \mathrm{~m}$ for the Trial02-2012 barn swallow data set under the body-fixed coordinate system with the constant speed maneuver.

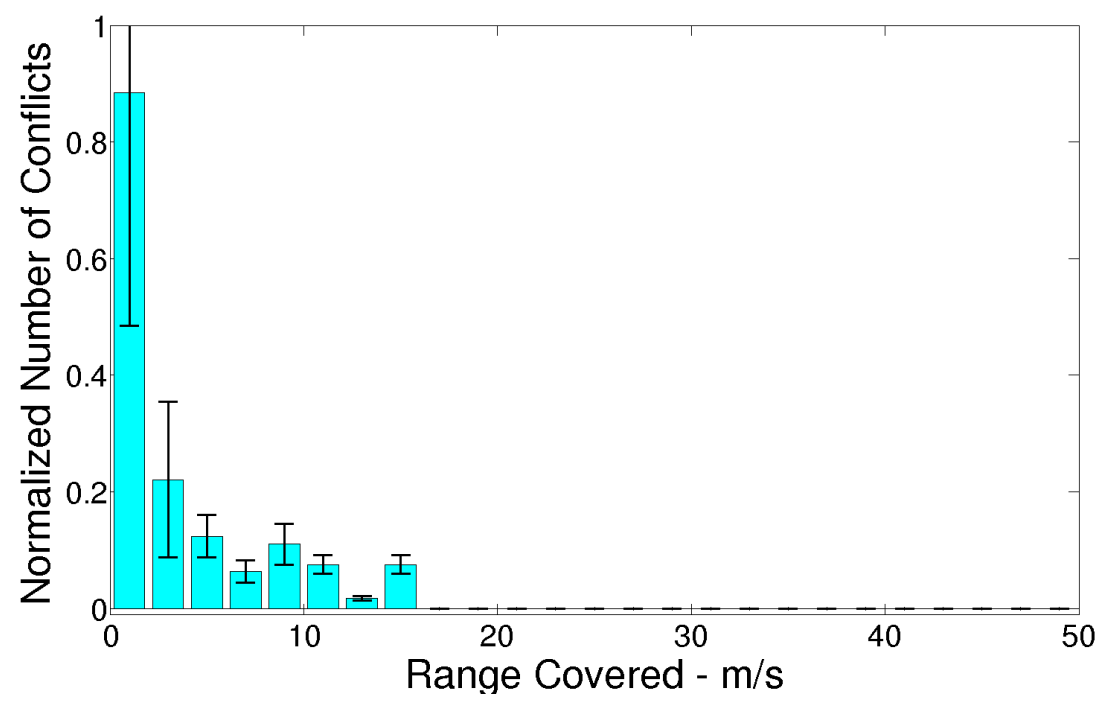

Figure 5.131: The $\Delta V$ distribution for three influential neighbors and $\delta_{\text {attraction }}=$ infinity for the Flock barn swallow data set under the body-fixed coordinate system with the variable speed maneuver. 


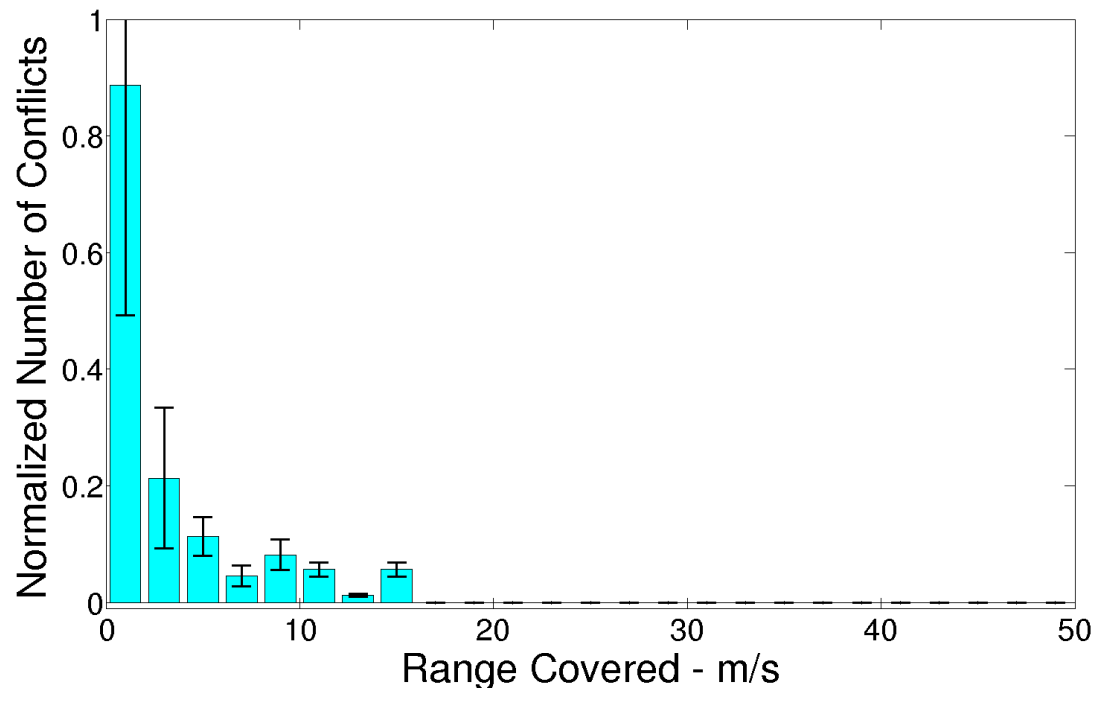

Figure 5.132: The $\Delta V$ distribution for seven influential neighbors and $\delta_{\text {attraction }}=$ infinity for the Flock barn swallow data set under the body-fixed coordinate system with the variable speed maneuver.

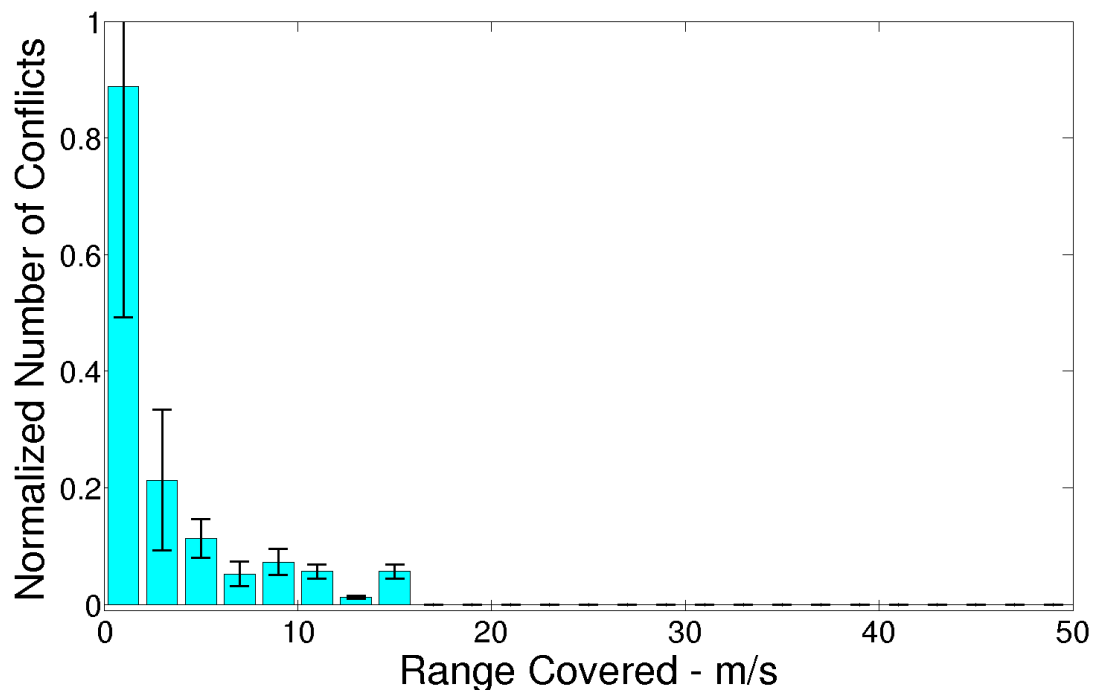

Figure 5.133: The $\Delta V$ distribution for the maximum number of influential neighbors and $\delta_{\text {attraction }}=$ infinity for the Flock barn swallow data set under the body-fixed coordinate system with the variable speed maneuver. 


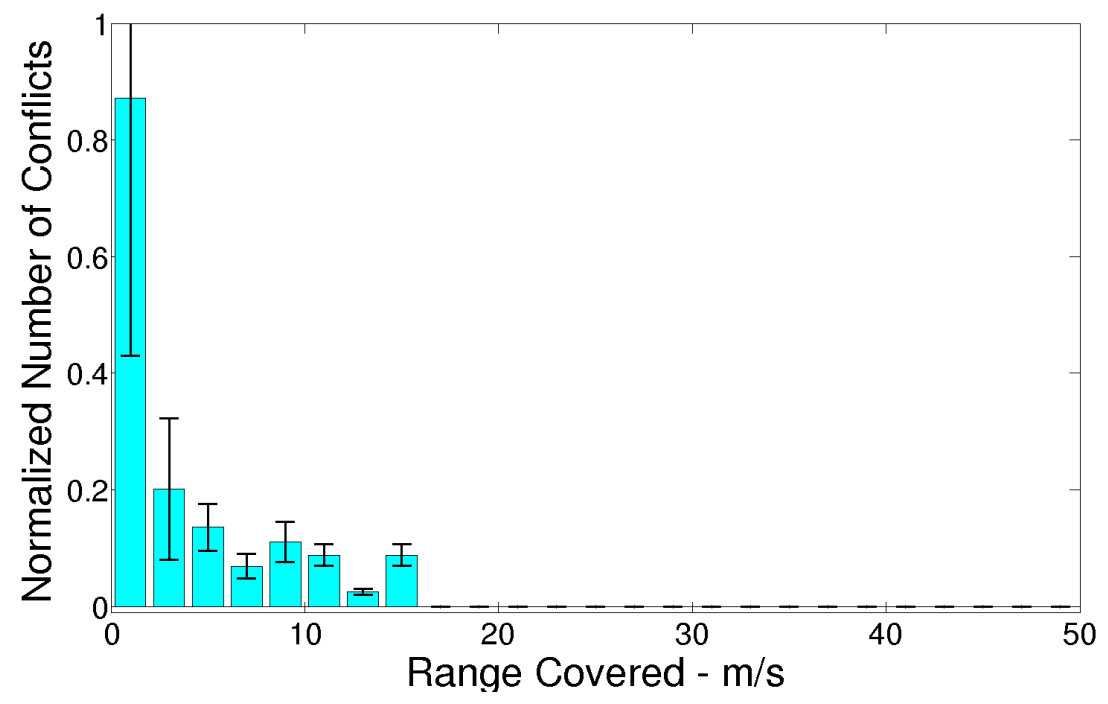

Figure 5.134: The $\Delta V$ distribution for seven influential neighbors and $\delta_{\text {attraction }}=5 \mathrm{~m}$ for the Flock barn swallow data set under the body-fixed coordinate system with the variable speed maneuver.

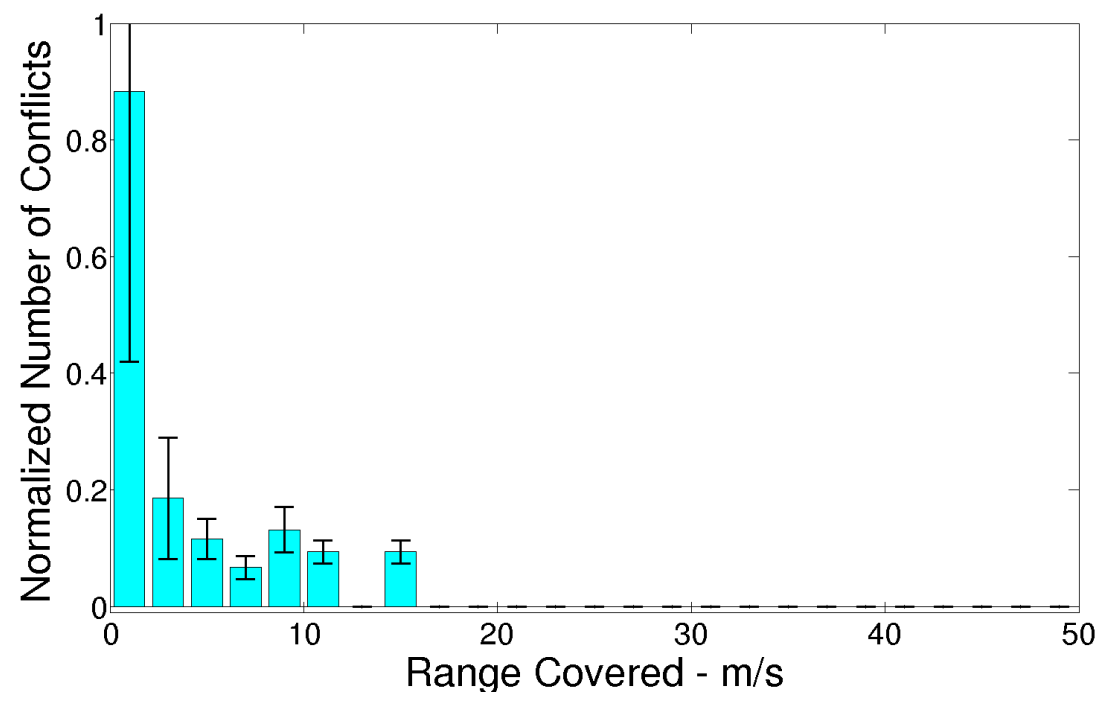

Figure 5.135: The $\Delta V$ distribution for seven influential neighbors and $\delta_{\text {attraction }}=3 \mathrm{~m}$ for the Flock barn swallow data set under the body-fixed coordinate system with the variable speed maneuver. 


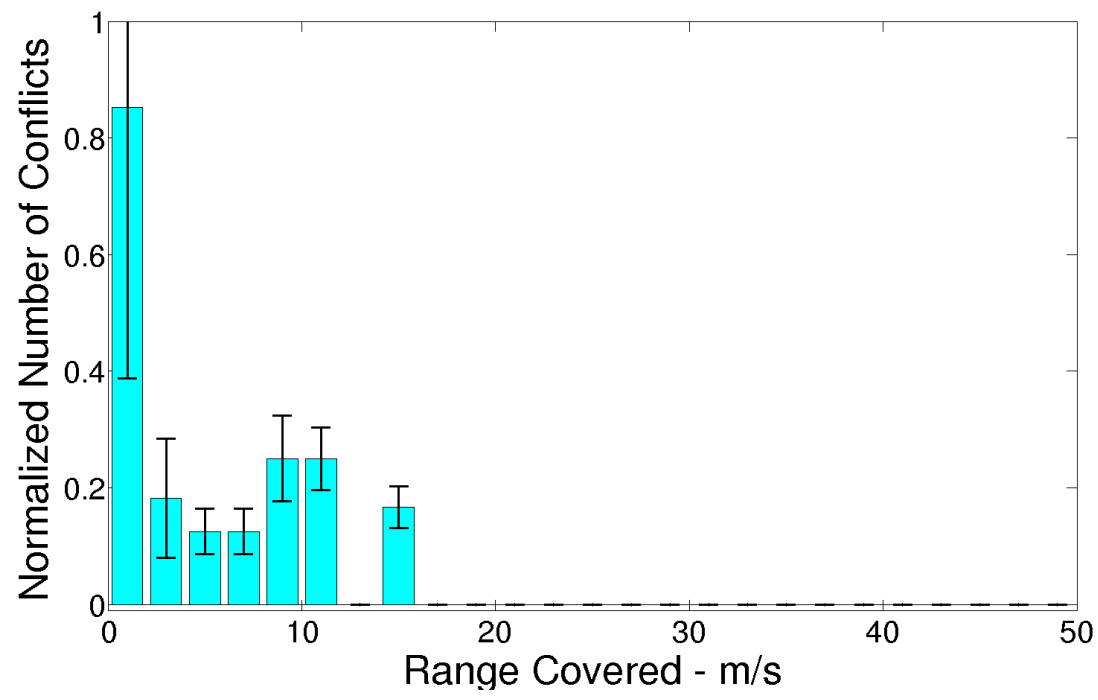

Figure 5.136: The $\Delta V$ distribution for seven influential neighbors and $\delta_{\text {attraction }}=1.5 \mathrm{~m}$ for the Flock barn swallow data set under the body-fixed coordinate system with the variable speed maneuver.

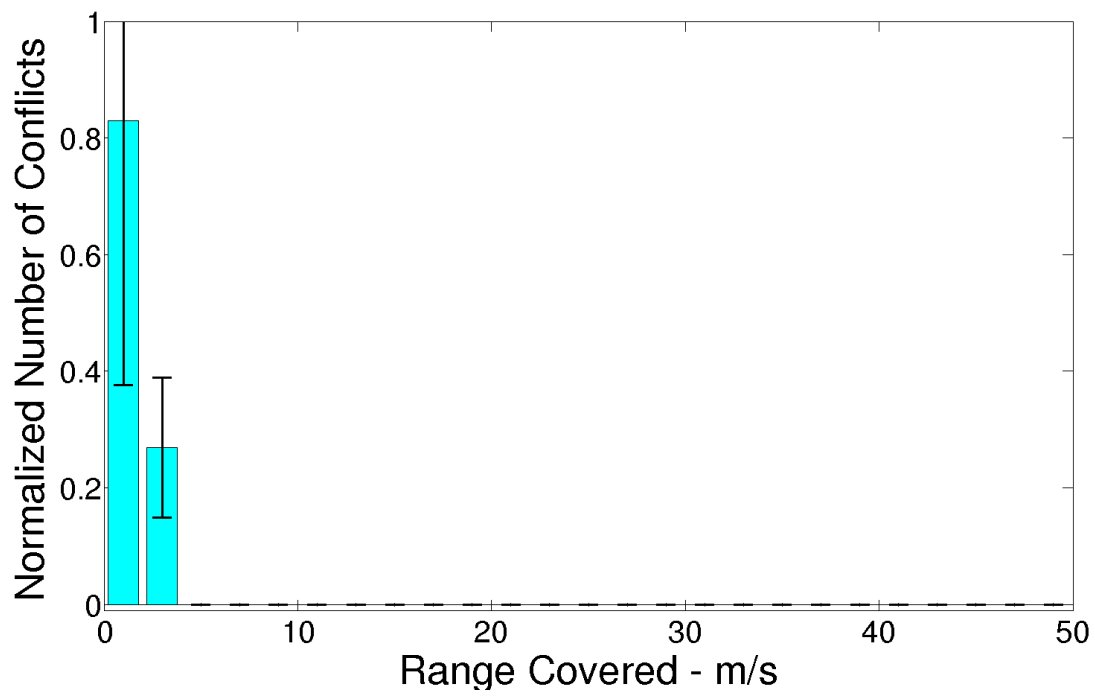

Figure 5.137: The $\Delta V$ distribution for three influential neighbors and $\delta_{\text {attraction }}=$ infinity for the Trial03 barn swallow data set under the body-fixed coordinate system with the variable speed maneuver. 


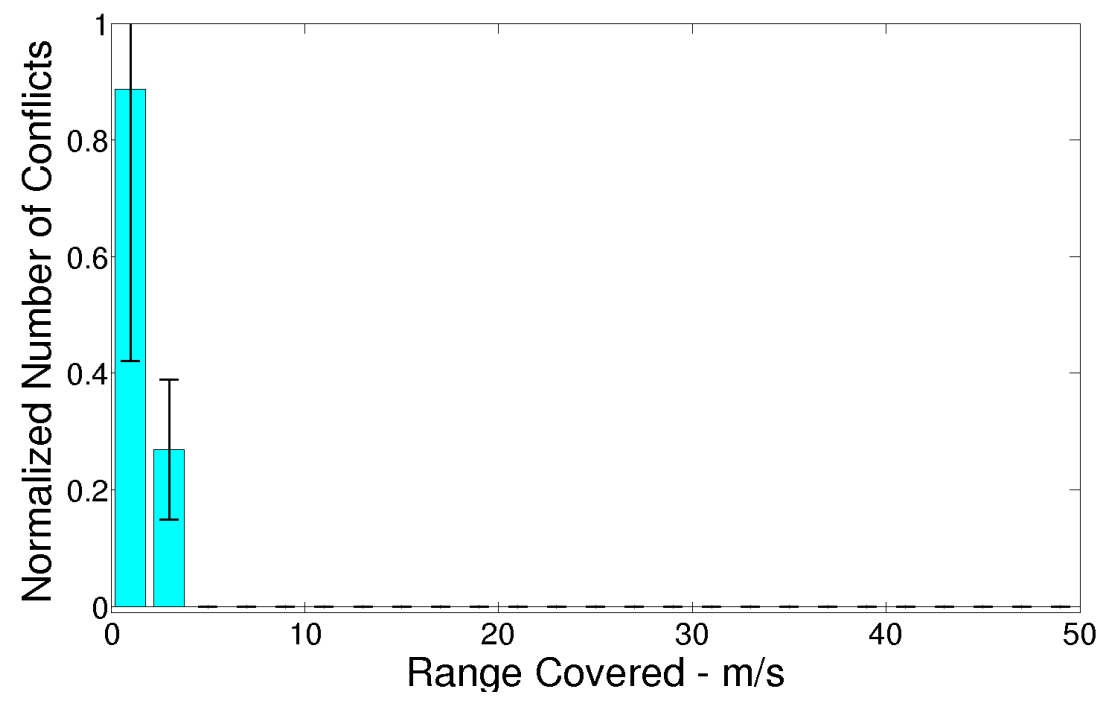

Figure 5.138: The $\Delta V$ distribution for seven influential neighbors and $\delta_{\text {attraction }}=$ infinity for the Trial03 barn swallow data set under the body-fixed coordinate system with the variable speed maneuver.

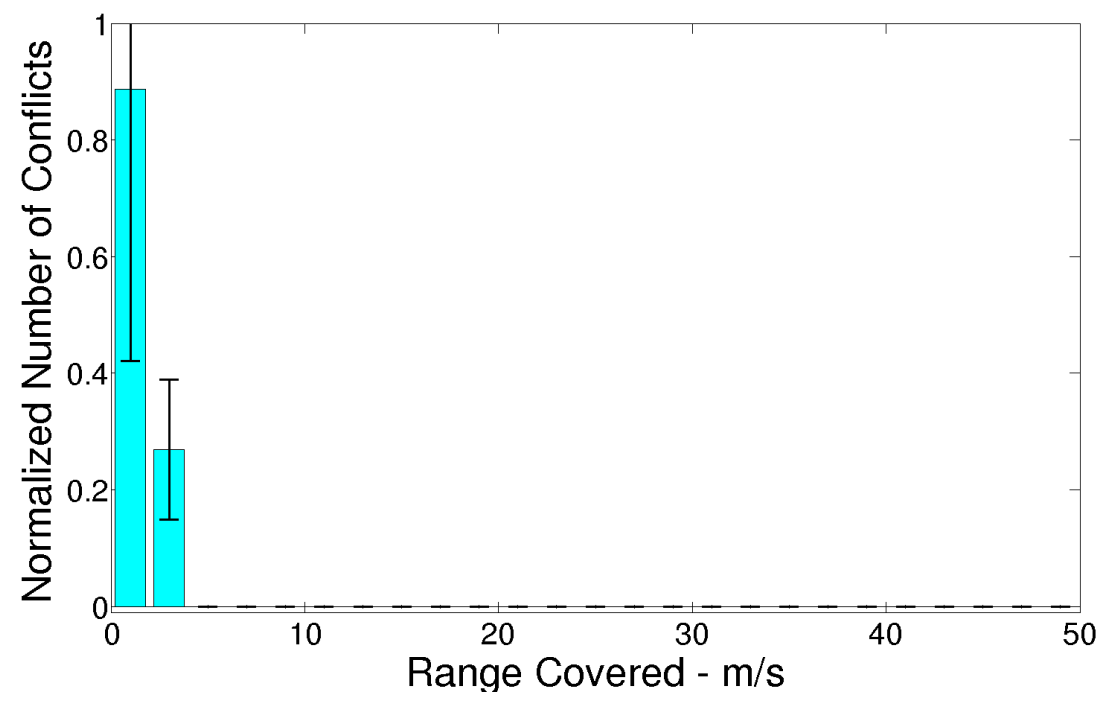

Figure 5.139: The $\Delta V$ distribution for the maximum number of influential neighbors and $\delta_{\text {attraction }}=$ infinity for the Trial03 barn swallow data set under the body-fixed coordinate system with the variable speed maneuver. 


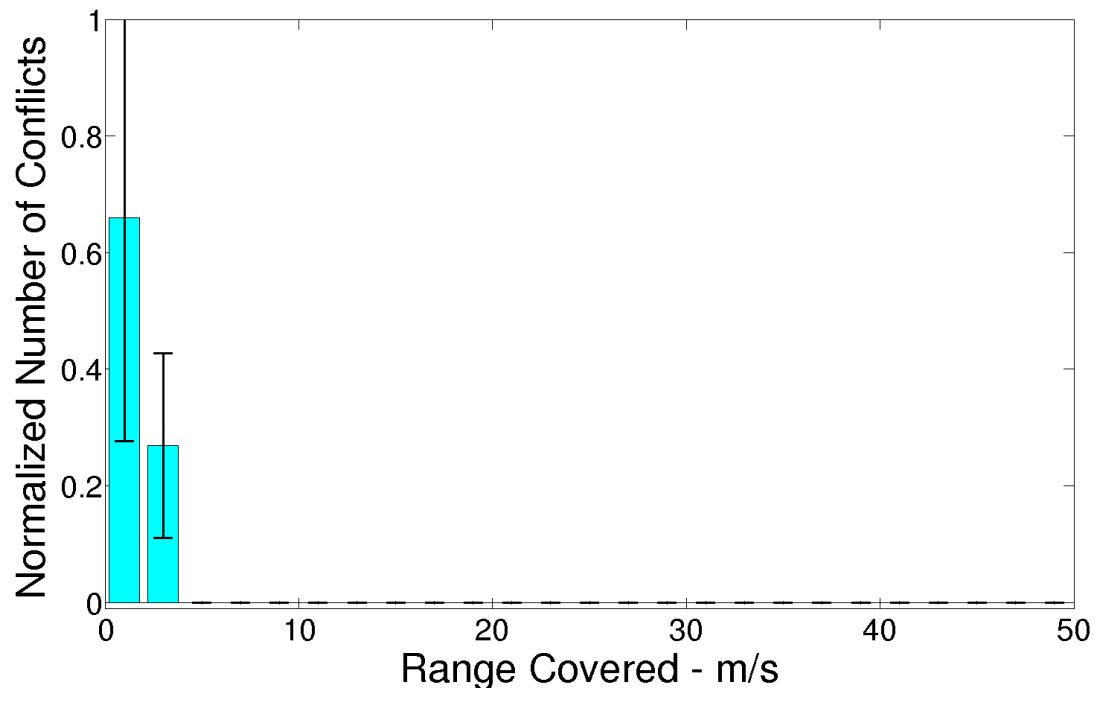

Figure 5.140: The $\Delta V$ distribution for seven influential neighbors and $\delta_{\text {attraction }}=5 \mathrm{~m}$ for the Trial03 barn swallow data set under the body-fixed coordinate system with the variable speed maneuver.

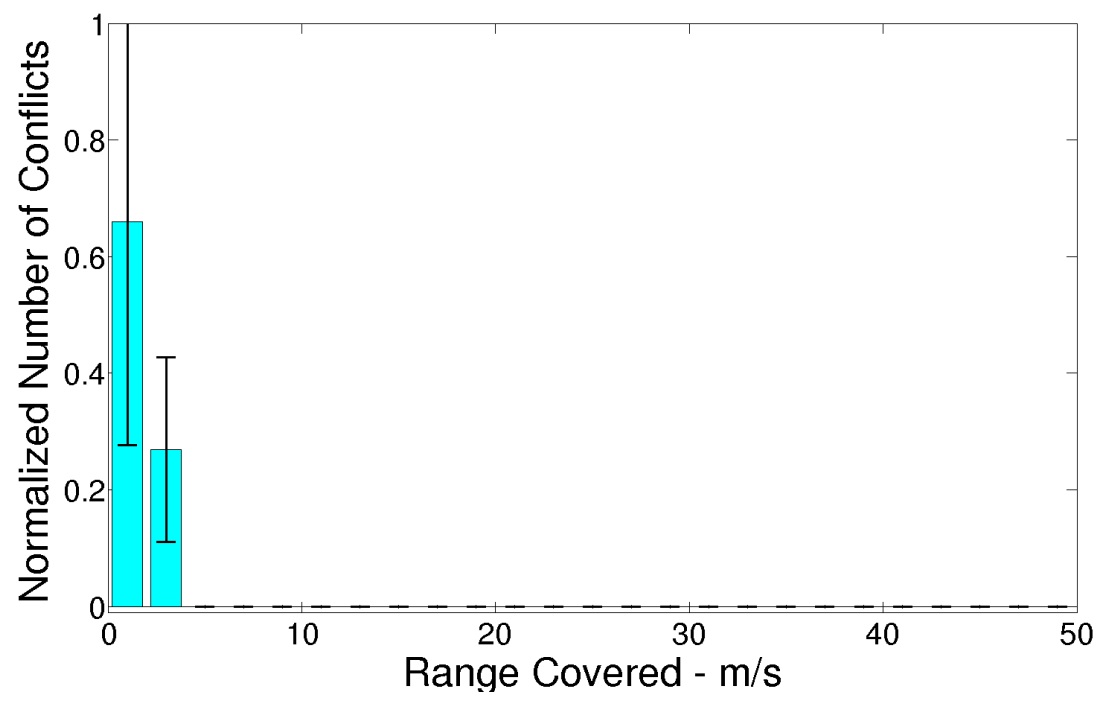

Figure 5.141: The $\Delta V$ distribution for seven influential neighbors and $\delta_{\text {attraction }}=3 \mathrm{~m}$ for the Trial03 barn swallow data set under the body-fixed coordinate system with the variable speed maneuver. 


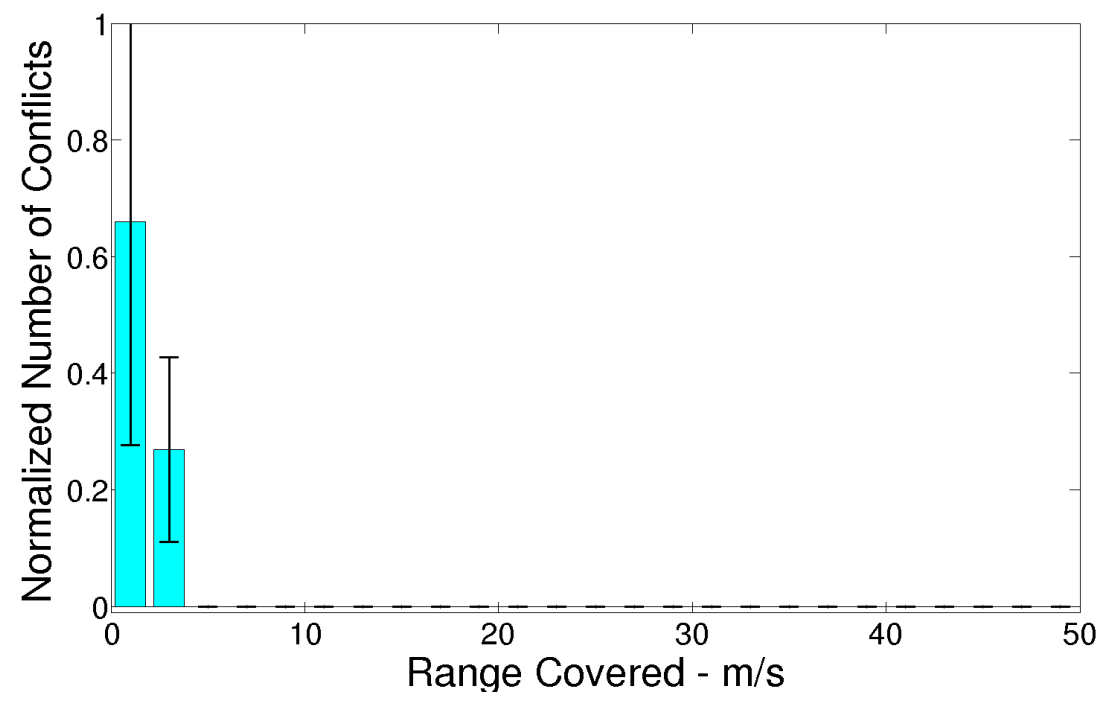

Figure 5.142: The $\Delta V$ distribution for seven influential neighbors and $\delta_{\text {attraction }}=1.5 \mathrm{~m}$ for the Trial03 barn swallow data set under the body-fixed coordinate system with the variable speed maneuver.

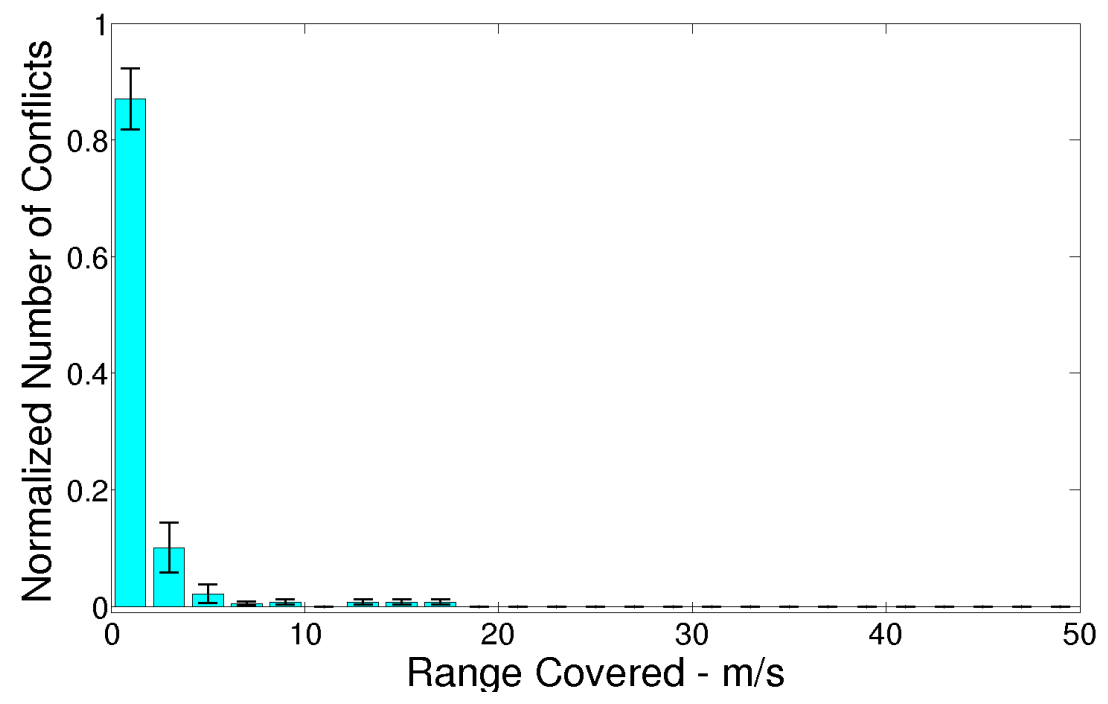

Figure 5.143: The $\Delta V$ distribution for three influential neighbors and $\delta_{\text {attraction }}=$ infinity for the Trial04 barn swallow data set under the body-fixed coordinate system with the variable speed maneuver. 


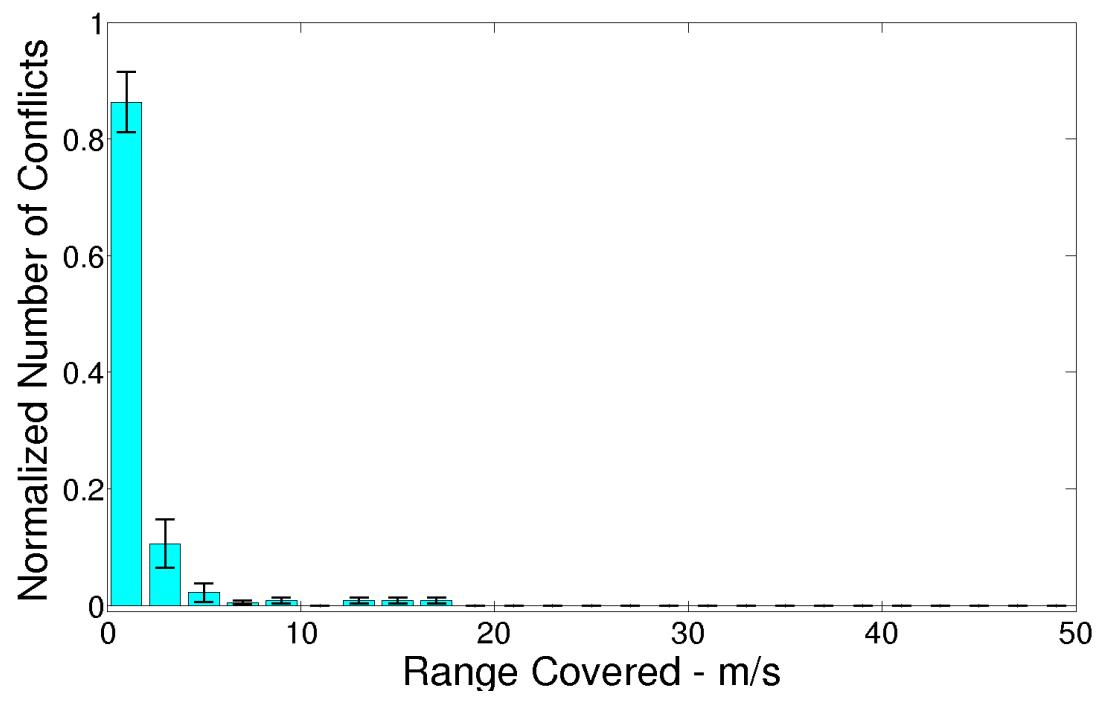

Figure 5.144: The $\Delta V$ distribution for three influential neighbors and $\delta_{\text {attraction }}=5 \mathrm{~m}$ for the Trial04 barn swallow data set under the body-fixed coordinate system with the variable speed maneuver.

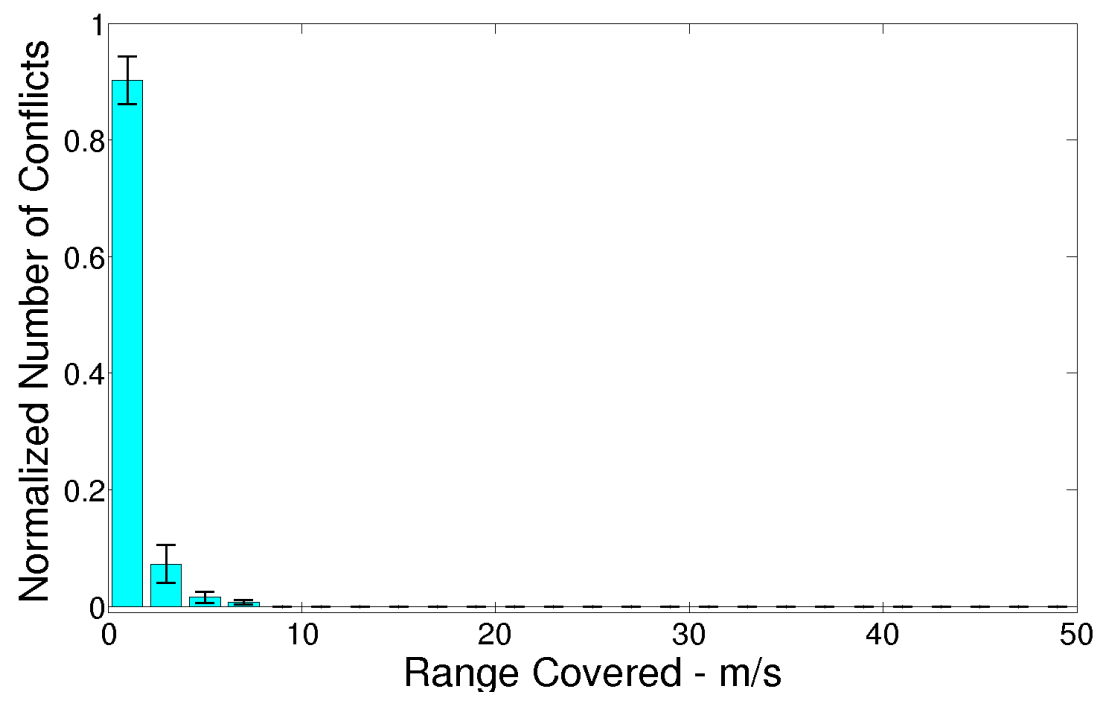

Figure 5.145: The $\Delta V$ distribution for three influential neighbors and $\delta_{\text {attraction }}=3 \mathrm{~m}$ for the Trial04 barn swallow data set under the body-fixed coordinate system with the variable speed maneuver. 


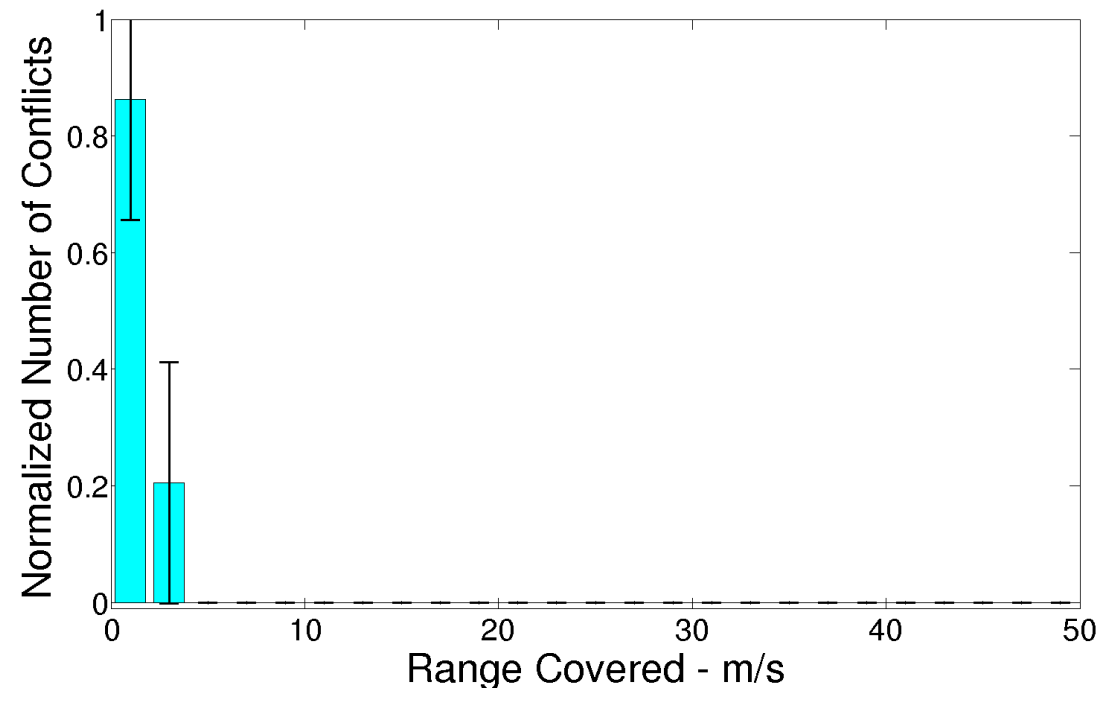

Figure 5.146: The $\Delta V$ distribution for three influential neighbors and $\delta_{\text {attraction }}=1.5 \mathrm{~m}$ for the Trial04 barn swallow data set under the body-fixed coordinate system with the variable speed maneuver.

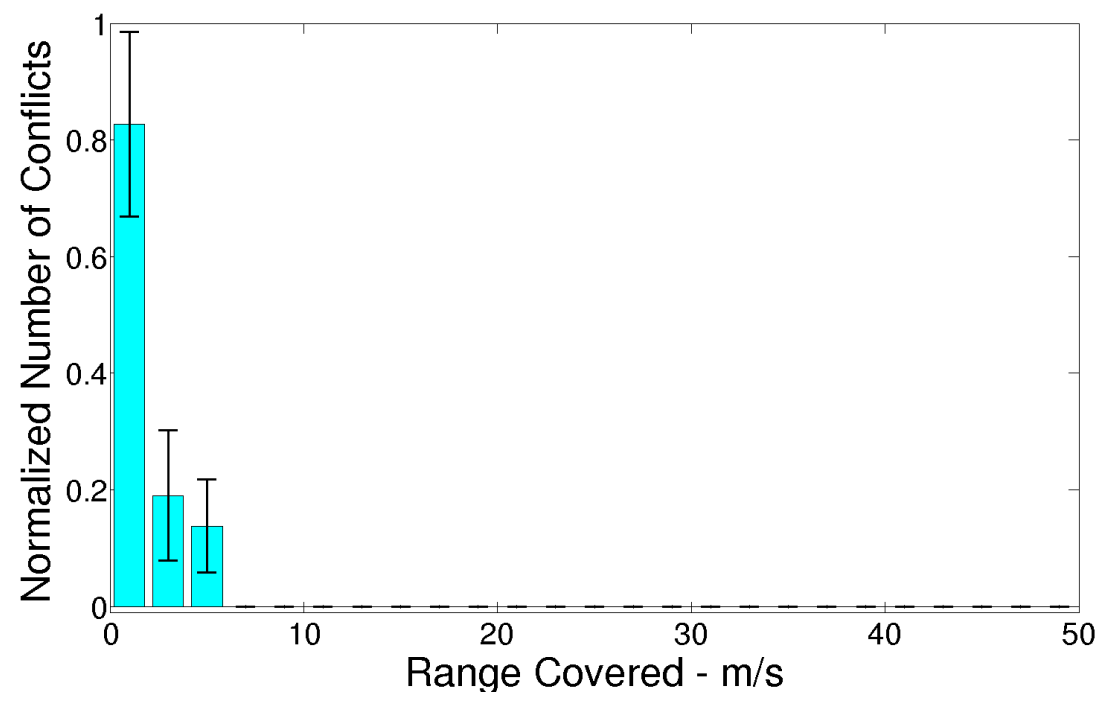

Figure 5.147: The $\Delta V$ distribution for three influential neighbors and $\delta_{\text {attraction }}=$ infinity for the Trial02-2012 barn swallow data set under the body-fixed coordinate system with the variable speed maneuver. 


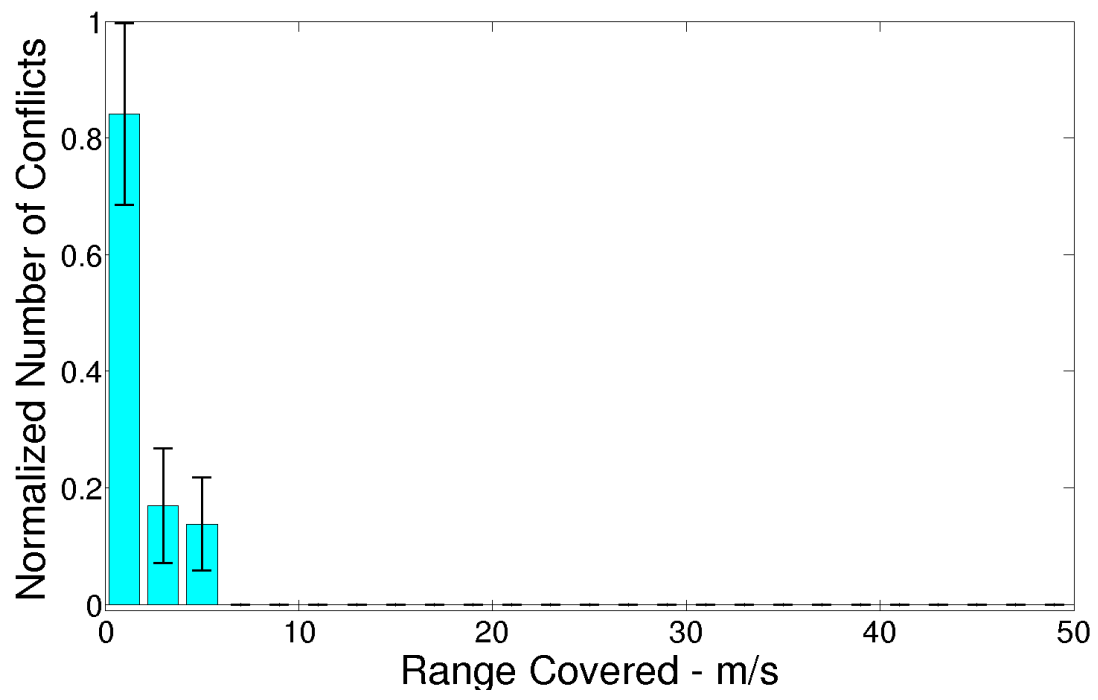

Figure 5.148: The $\Delta V$ distribution for three influential neighbors and $\delta_{\text {attraction }}=5 \mathrm{~m}$ for the Trial02-2012 barn swallow data set under the body-fixed coordinate system with the variable speed maneuver.

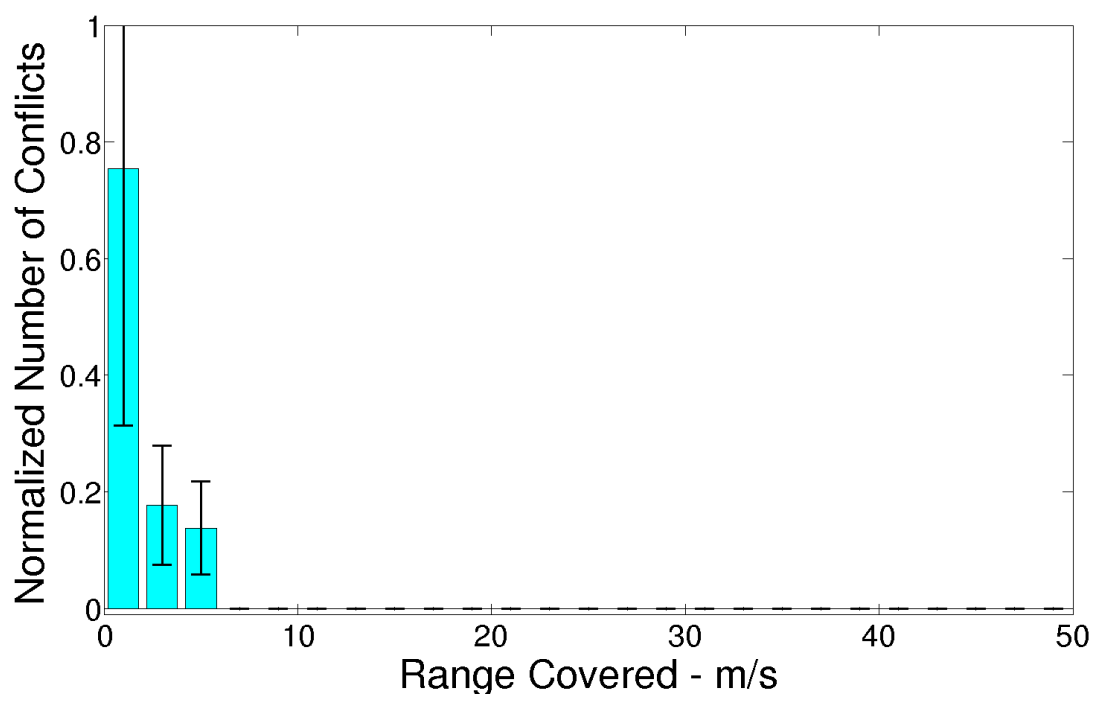

Figure 5.149: The $\Delta V$ distribution for three influential neighbors and $\delta_{\text {attraction }}=3 \mathrm{~m}$ for the Trial02-2012 barn swallow data set under the body-fixed coordinate system with the variable speed maneuver. 


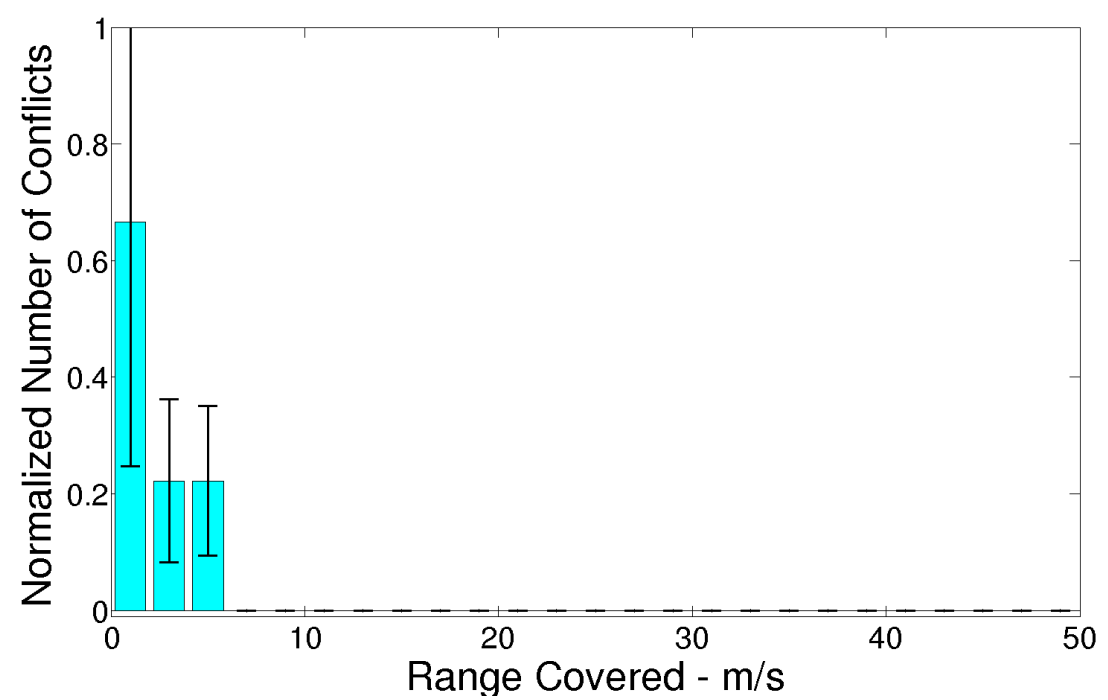

Figure 5.150: The $\Delta V$ distribution for three influential neighbors and $\delta_{\text {attraction }}=1.5 \mathrm{~m}$ for the Trial02-2012 barn swallow data set under the body-fixed coordinate system with the variable speed maneuver. 


\subsection{Cave Bats}

The values of $\delta_{\text {attraction }}$ used in the bat data analysis were $0.5 \mathrm{~m}, 1 \mathrm{~m}, 3 \mathrm{~m}$ and infinite. The number of influential nearest neighbors were chosen from 3,5, 7 and all. The $d_{s e p}$ value used was $0.135 \mathrm{~m}$ for fellow bats and $0.09 \mathrm{~m}$ for the static obstacles. These values were chosen based on the wingspan of the bats. The maximum velocity of the bats was chosen to be 20 $\mathrm{m} / \mathrm{s}$ [13]. For analysis, the average $\Delta V$ was calculated and the distribution plotted. Each bin in the distribution had a width of $4 \mathrm{~m} / \mathrm{s}$.

\subsubsection{Global Coordinates}

First, the global coordinate frame was analyzed for both the constant and variable speed maneuver.

\section{Constant Speed Maneuver}

To begin, the analysis of the global coordinate frame constant speed maneuver, $\delta_{\text {attraction }}$ was held constant at infinity, and the number of influential nearest neighbors was varied, Figures 5.151-5.154. The results showed little change between the different number of influential nearest neighbors. A $\Delta V$ of less than $4 \mathrm{~m} / \mathrm{s}$ occured just over $60 \%$ of the time between the data and algorithm. The next bin, containing the $\Delta V$ values between 4 and $8 \mathrm{~m} / \mathrm{s}$ dropped down to occurring less than $20 \%$ of the time. Table 5.2 contained the average number of frames in which an individual bat experienceed at least one conflict for the global coordinate constant speed scenario. The table showed a greater increase in the number of conflicts when increasing the maximum sensing range than it did when increasing the number of influential neighbors. Table 5.3 contains the average time, in seconds, a bat took to become conflict free. This reaction time was fairly consistent for all of the parameter variations at about 2.3 seconds.

\section{Variable Speed Maneuver}

Holding $\delta_{\text {attraction }}$ at infinity and varying the number of influential nearest neighbors produced an average $\Delta V$ of less than $4 \mathrm{~m} / \mathrm{s}$ just over $60 \%$ of the time, Figures 5.155-5.158. 
The 4 to $8 \mathrm{~m} / \mathrm{s}$ range of $\Delta V$ occured less than $20 \%$ of the time. This immediate decay and subsequent decays are an indicator of the small standard deviation of $\Delta V$. Table 5.4 contains the average number of frames for which an individual bat experienced at least one conflict for the global coordinate variable speed scenario. The table showed a greater increase in the number of conflicts when increasing the maximum sensing range than it did when increasing the number of influential neighbors. Table 5.5 contains the average time, in seconds, a bat took to become conflict free. This reaction time was fairly consistent for all the parameter variations at about 2.3 seconds. While the algorithm did not fit poorly with how the bats move, it was not as highly correlated as in some of the cases of the other two species.

\subsubsection{Body Coordinates}

\section{Constant Speed Maneuver}

First, $\delta_{\text {attraction }}$ was held constant at infinity, and the number of influential nearest neighbors was varied (Figures 5.159-5.162). The distributions showed an improvement from the global coordinate frame. The average $\Delta V$ values were less than $4 \mathrm{~m} / \mathrm{s}$ approximately $70 \%$ of the time for the entire range of influential nearest neighbors. The value of $\Delta V$ was between 4 and $8 \mathrm{~m} / \mathrm{s}$ less than $20 \%$ of the time.

Next, the number of influential nearest neighbors was held constant at the maximal value,

and $\delta_{\text {attraction }}$ was varied through all its values (Figures 5.163-5.165). For $\delta_{\text {attraction }}=3 \mathrm{~m}$, the distribution showed an average $\Delta V$ less than $4 \mathrm{~m} / \mathrm{s}$ occurring just over $70 \%$ of the time and between 4 and $8 \mathrm{~m} / \mathrm{s}$ less than $20 \%$ of the time. As $\delta_{\text {attraction }}$ decreased, the correlation decreases. For $\delta_{\text {attraction }}=1 \mathrm{~m}, \Delta V$ was less than $4 \mathrm{~m} / \mathrm{s}$ approximately $50 \%$ of the time and was within $4-8 \mathrm{~m} / \mathrm{s}$ just over $10 \%$ of the time. When looking at the $\Delta V$ distribution for $\delta_{\text {attraction }}=0.5 \mathrm{~m}$, the less than $4 \mathrm{~m} / \mathrm{s}$ bin was between $30 \%$ and $40 \%$ which does not indicate a strong correlation between data and algorithm.

From these results, the inference can be made that the best fitting parameters for the constant speed maneuver in body coordinates were seven influential nearest neighbors and $\delta_{\text {attraction }}=3 \mathrm{~m}$. Figure 5.166 shows the distribution with the results for this particular set of 
parameters. The average $\Delta V$ was less than $4 \mathrm{~m} / \mathrm{s}$ over $70 \%$ of the time and between 4 and 8 $\mathrm{m} / \mathrm{s}$ less than $20 \%$ of the time. This result was comparable to the best bat results seen above for the body coordinate constant speed maneuver. This algorithm with the parameters of $\delta_{\text {attraction }}=3 \mathrm{~m}$ and seven influential nearest neighbors, correlated well with what bats did to avoid collisions in the data presented here. Table 5.6 contains the average number of frames in which an individual bat experienced at least one conflict for the body coordinate constant speed scenario. The table showed a greater increase in the number of conflicts when increasing the maximum sensing range than it did when increasing the number of influential neighbors. Table 5.7 contains the average time, in seconds, bats took to become conflict free. This reaction time was fairly consistent for all the parameter variations at about 2.3 seconds.

\section{Variable Speed Maneuver}

Lastly, the body coordinate frame variable speed maneuver was analyzed. Varying the number of influential nearest neighbors and keeping $\delta_{\text {attraction }}$ constant at infinity produced the distributions in Figures 5.167-5.170. The distributions showed a slight change with respect to the number of influential nearest neighbors. The distributions for three and five influential nearest neighbors showed that the average $\Delta V$ was less $4 \mathrm{~m} / \mathrm{s}$ about $70 \%$ of the time, while the seven and all nearest neighbors scenarios showed this bin being just above $70 \%$. To determine the best value of $\delta_{\text {attraction }}$, the number of influential nearest neighbors was set to the maximal value while $\delta_{\text {attraction }}$ was varied (Figures 5.171-5.173). For $\delta_{\text {attraction }}=3 \mathrm{~m}$, Figure $5.163, \Delta V$ was less than $4 \mathrm{~m} / \mathrm{s}$ over $70 \%$ of the time. As $\delta_{\text {attraction }}$ decreased, the percent of the time that $\Delta V$ was less than $4 \mathrm{~m} / \mathrm{s}$ decreased. For $\delta_{\text {attraction }}=1 \mathrm{~m}$ the correlation was just above $50 \%$, and for $\delta_{\text {attraction }}=0.5 \mathrm{~m}$, it was just above $30 \%$; neither result was particularly strong. Table 5.8 contains the average number of frames for which an individual bat experienced at least one conflict for the body coordinate variable speed scenario. The table shows a greater increase in the number of conflicts when increasing the maximum sensing range than it does when increasing the number of influential neighbors. Table 5.9 contains the average time, in seconds, a bat needed to become conflict 


\begin{tabular}{cc|cccc}
\hline & & \multicolumn{4}{|c}{ Maximum Sensing Range } \\
& & $0.5 \mathrm{~m}$ & $1 \mathrm{~m}$ & $3 \mathrm{~m}$ & infinite \\
\hline \multirow{2}{*}{ Number of } & 5 & 8.92 & 15.93 & 22.20 & 23.20 \\
Influencial Neighbors & 7 & 8.92 & 16.67 & 26.35 & 29.20 \\
& All & 8.92 & 16.67 & 28.60 & 32.80 \\
& & & 28.80 & 36.40 \\
\hline
\end{tabular}

Table 5.2: The average number of frames with at least one conflict seen by an individual bat in the global coordinate frame for a constant speed maneuver. The top parameter is the maximum sensing range in meters, and the left parameter is the number of neighbors being sensed. A total of 1179 frames were used in the analysis.

free. This reaction time was fairly consistent for all the parameter variations at about 2.3 seconds.

From the results above, the best parameters for the body frame variable speed maneuver were $\delta_{\text {attraction }}=3 \mathrm{~m}$ and seven influential nearest neighbors. The distribution corresponding to these parameters, Figure 5.174 , showed that the average $\Delta V$ was less than $4 \mathrm{~m} / \mathrm{s}$ over $70 \%$ of the time and between 4 and $8 \mathrm{~m} / \mathrm{s}$ less than $20 \%$ of the time.

\subsubsection{Discussion of Bat Data Analysis}

While the body frame algorithms showed stronger correlation relative to over the global coordinate frame maneuvers, the variable speed did not show an improvement over the constant speed. The variable speed having a higher correlation than the constant speed maneuver suggests that, relative to the algorithm considered here, the bats plan maneuvers in the body coordinate frame. The negligible differences in results for variable speed relative to constant speed maneuvers suggests that bats do not change their speed as much as they do their heading when avoiding collisions. The analysis of the cave bat reaction time consistently yielded an average of 2.3s. 


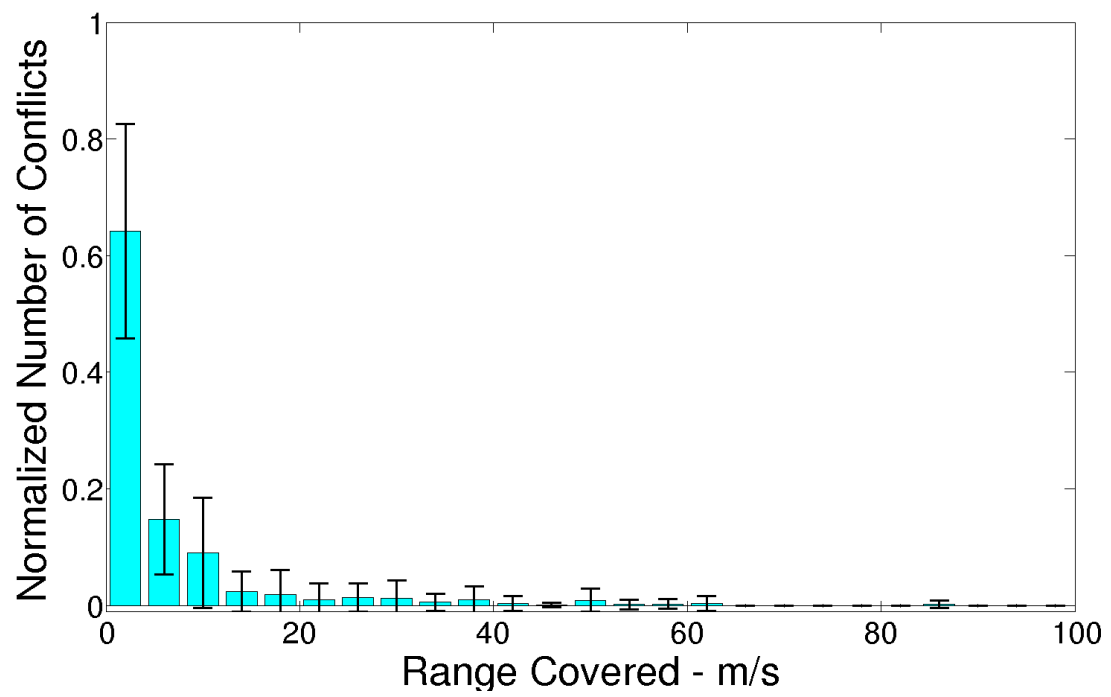

Figure 5.151: The $\Delta V$ distribution for three influential neighbors and $\delta_{\text {attraction }}=$ infinity for the bat data set under the global coordinate system with the constant speed maneuver.

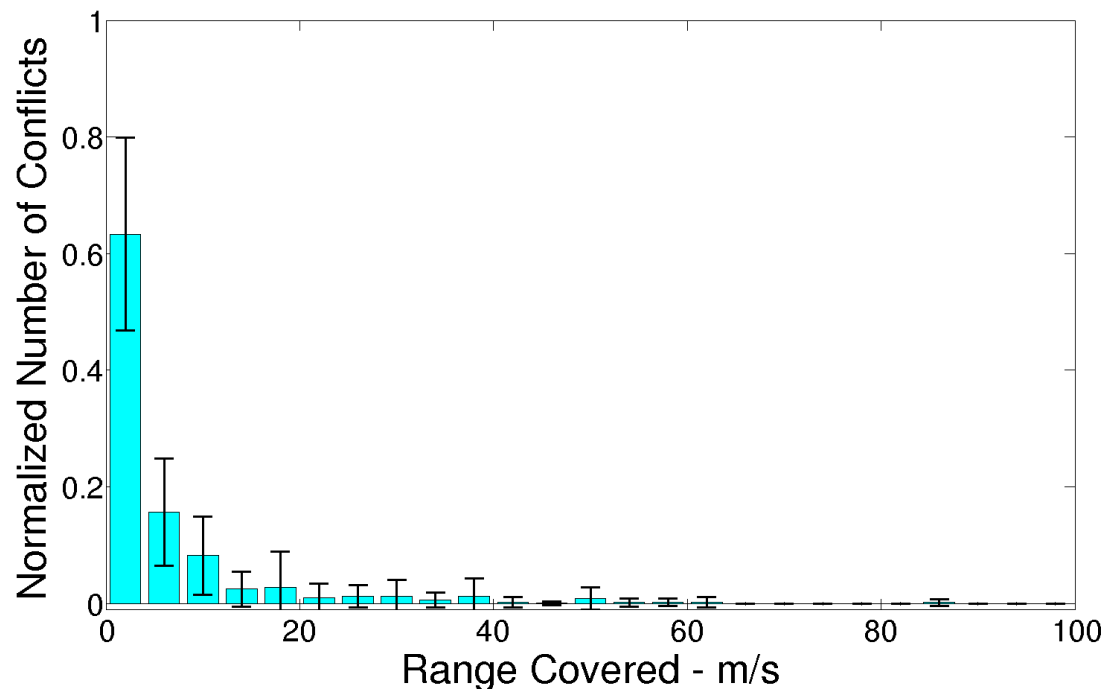

Figure 5.152: The $\Delta V$ distribution for five influential neighbors and $\delta_{\text {attraction }}=$ infinity for the bat data set under the global coordinate system with the constant speed maneuver. 


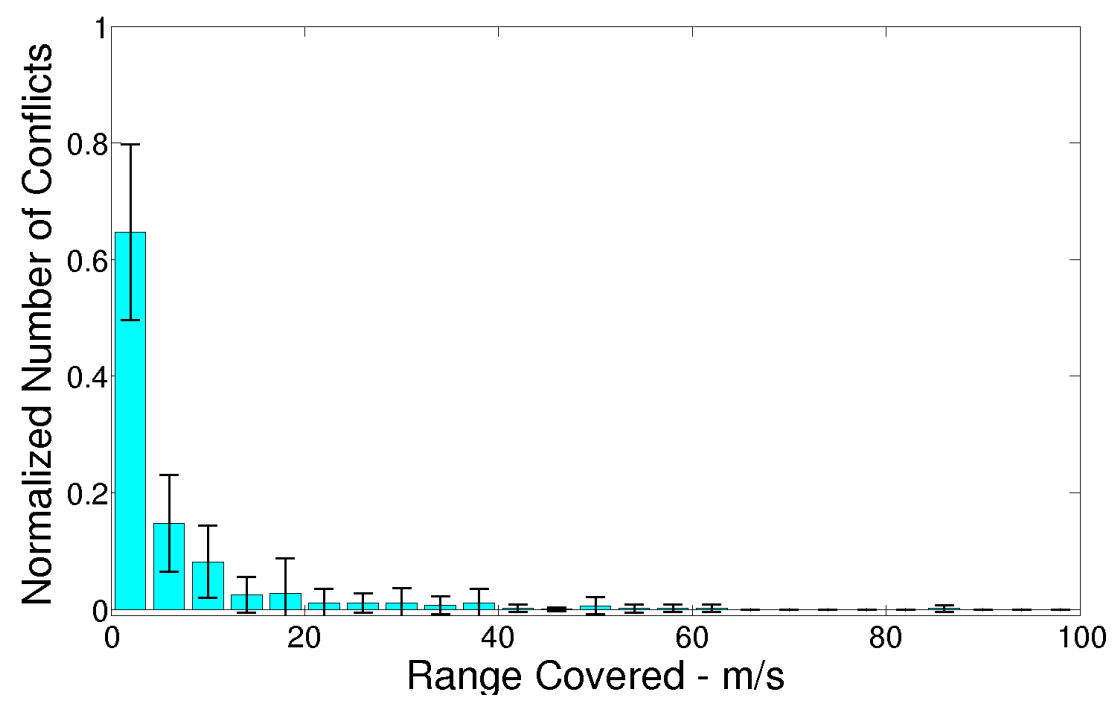

Figure 5.153: The $\Delta V$ distribution for seven influential neighbors and $\delta_{\text {attraction }}=$ infinity for the bat data set under the global coordinate system with the constant speed maneuver.

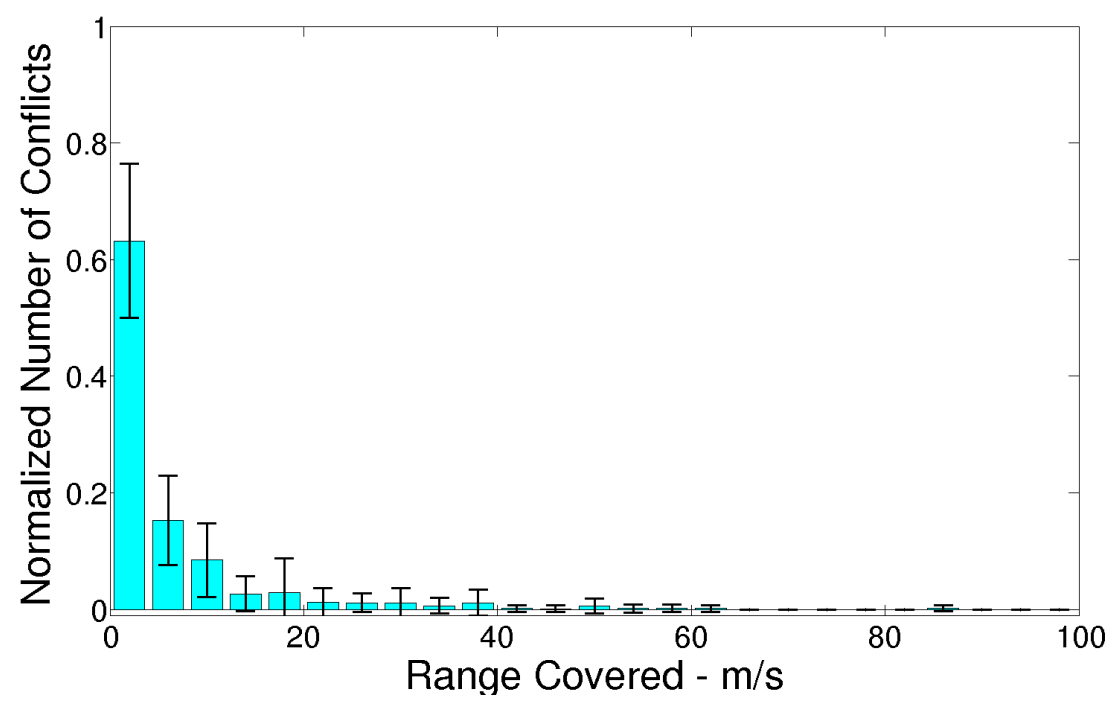

Figure 5.154: The $\Delta V$ distribution for the maximum number of influential neighbors and $\delta_{\text {attraction }}=$ infinity for the bat data set under the global coordinate system with the constant speed maneuver. 


\begin{tabular}{cl|cccc}
\hline & & \multicolumn{4}{|c}{ Maximum Sensing Range } \\
& 3 & $0.5 \mathrm{~m}$ & $1 \mathrm{~m}$ & $3 \mathrm{~m}$ & infinite \\
\hline \multirow{2}{*}{ Number of } & 5 & $2.29 \mathrm{~s}$ & $2.29 \mathrm{~s}$ & $2.33 \mathrm{~s}$ & $2.33 \mathrm{~s}$ \\
Influential Neighbors & 7 & $2.29 \mathrm{~s}$ & $2.33 \mathrm{~s}$ & $2.44 \mathrm{~s}$ \\
& All & $2.29 \mathrm{~s}$ & $2.29 \mathrm{~s}$ & $2.33 \mathrm{~s}$ & $2.44 \mathrm{~s}$ \\
& & $2.29 \mathrm{~s}$ & $2.33 \mathrm{~s}$ & $2.44 \mathrm{~s}$ \\
\hline
\end{tabular}

Table 5.3: The average time, in seconds, in the global coordinate frame scenario with the constant speed maneuver for an individual bat to shift its velocity vector closest to that of the optimal. The top parameter is the maximum sensing range in meters, and the left parameter is the number of neighbors being sensed.

\begin{tabular}{cc|cccc}
\hline & & \multicolumn{4}{|c}{ Maximum Sensing Range } \\
& & $0.5 \mathrm{~m}$ & $1 \mathrm{~m}$ & $3 \mathrm{~m}$ & infinitye \\
\hline \multirow{2}{*}{ Number of } & 5 & 9.92 & 16.87 & 23.35 & 24.40 \\
Influential Neighbors & 7 & 9.92 & 17.60 & 27.50 & 30.40 \\
& & 9.92 & 17.60 & 29.80 & 34.15 \\
& All & 9.92 & 17.60 & 30.00 & 37.75 \\
\hline
\end{tabular}

Table 5.4: The average number of frames with at least one conflict seen by an individual bat in the global coordinate frame for a variable speed maneuver. The top parameter is the maximum sensing range in meters, and the left parameter is the number of neighbors being sensed. A total of 1179 frames are used in the analysis.

\begin{tabular}{cl|cccc}
\hline & & \multicolumn{4}{|c}{ Maximum Sensing Range } \\
& & $0.5 \mathrm{~m}$ & $1 \mathrm{~m}$ & $3 \mathrm{~m}$ & infinite \\
\hline \multirow{3}{*}{ Number of } & 5 & $2.29 \mathrm{~s}$ & $2.29 \mathrm{~s}$ & $2.33 \mathrm{~s}$ & $2.33 \mathrm{~s}$ \\
Influential Neighbors & 7 & $2.29 \mathrm{~s}$ & $2.29 \mathrm{~s}$ & $2.33 \mathrm{~s}$ & $2.44 \mathrm{~s}$ \\
& All & $2.29 \mathrm{~s}$ & $2.29 \mathrm{~s}$ & $2.33 \mathrm{~s}$ & $2.44 \mathrm{~s}$ \\
& & $2.29 \mathrm{~s}$ & $2.33 \mathrm{~s}$ & $2.44 \mathrm{~s}$ \\
\hline
\end{tabular}

Table 5.5: The average time, in seconds, in the global coordinate frame scenario with the variable speed maneuver for an indivual bat uses to shift its velocity vector closest to that of the optimal. The top parameter is the maximum sensing range in meters, and the left parameter is the number of neighbors being sensed. 


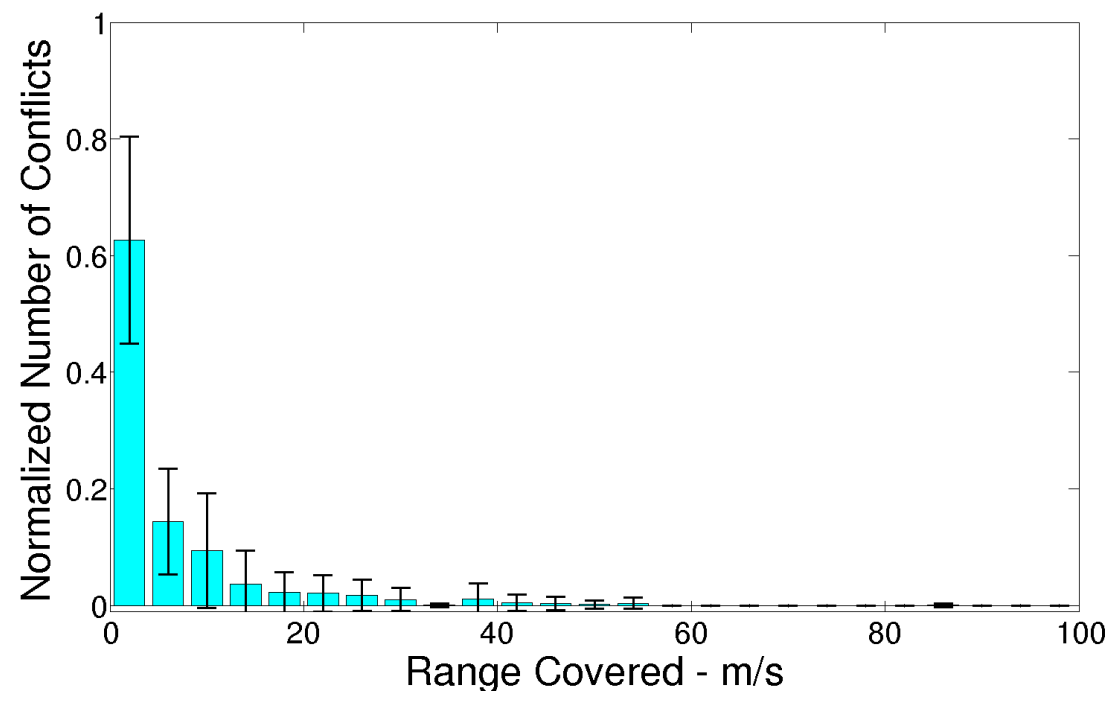

Figure 5.155: The $\Delta V$ distribution for three influential neighbors and $\delta_{\text {attraction }}=$ infinity for the bat data set under the global coordinate system with the variable speed maneuver.

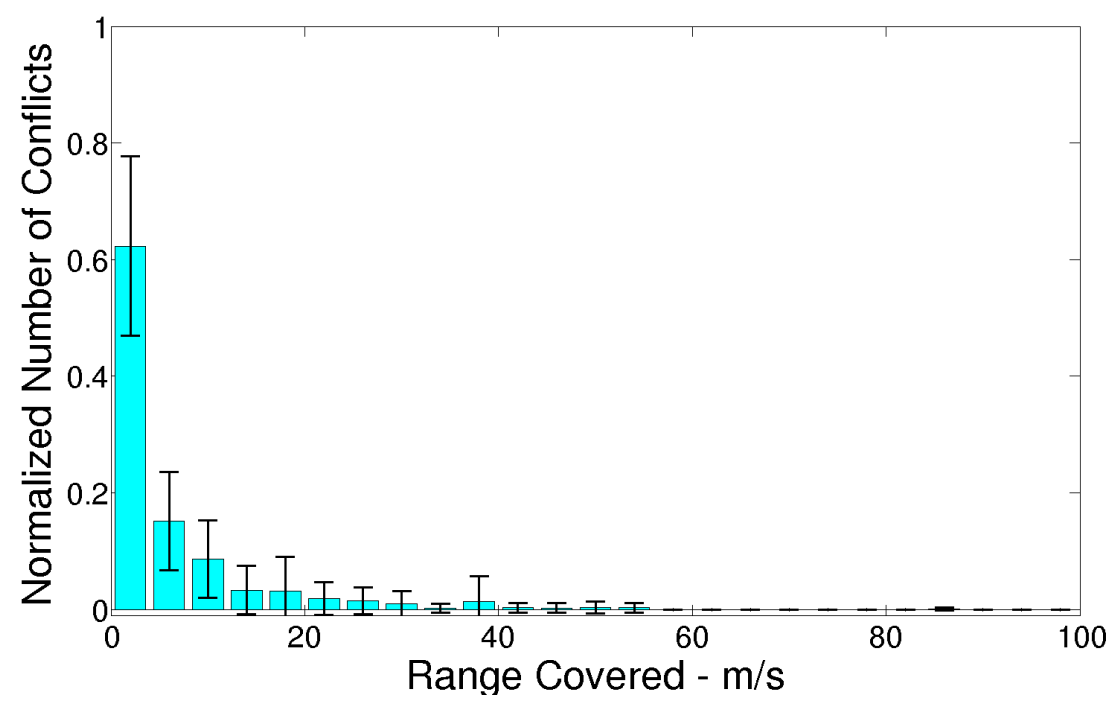

Figure 5.156: The $\Delta V$ distribution for five influential neighbors and $\delta_{\text {attraction }}=$ infinity for the bat data set under the global coordinate system with the variable speed maneuver. 


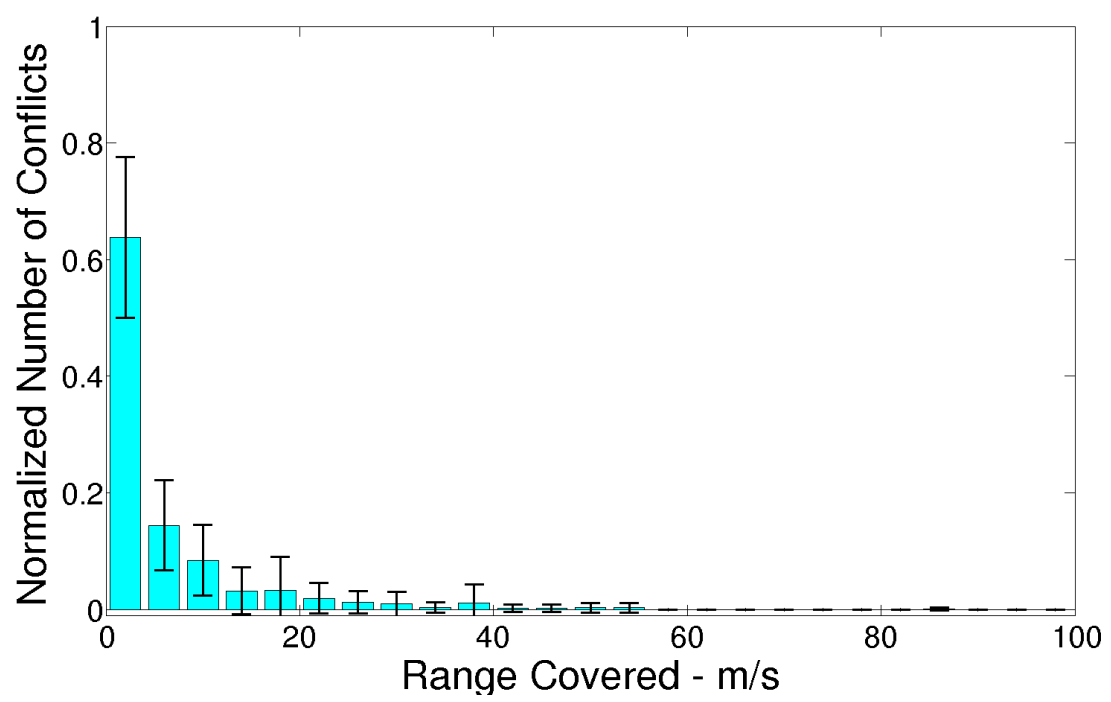

Figure 5.157: The $\Delta V$ distribution for seven influential neighbors and $\delta_{\text {attraction }}=$ infinity for the bat data set under the global coordinate system with the variable speed maneuver.

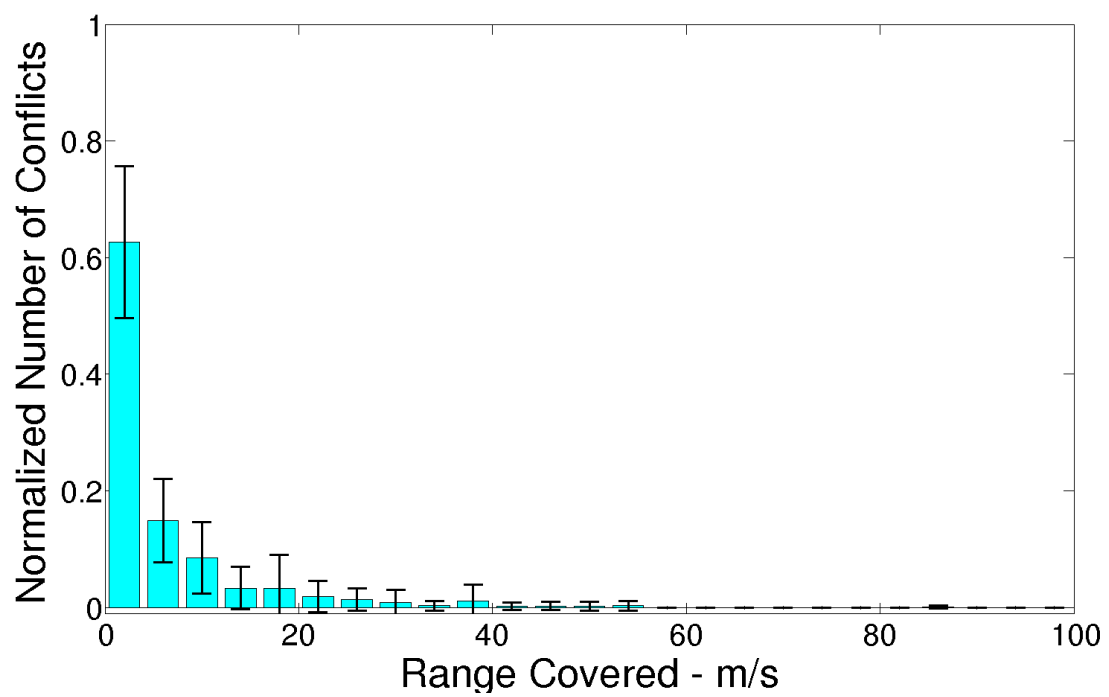

Figure 5.158: The $\Delta V$ distribution for the maximum number of influential neighbors and $\delta_{\text {attraction }}=$ infinity for the bat data set under the global coordinate system with the variable speed maneuver. 


\begin{tabular}{cc|cccc}
\hline & & \multicolumn{4}{|c}{ Maximum Sensing Range } \\
& & $0.5 \mathrm{~m}$ & $1 \mathrm{~m}$ & $3 \mathrm{~m}$ & infinite \\
\hline \multirow{3}{*}{ Number of } & 5 & 8.47 & 16.19 & 24.40 & 25.30 \\
Influential Neighbors & 7 & 8.47 & 16.75 & 28.00 & 30.30 \\
& All & 8.47 & 16.88 & 30.50 & 34.00 \\
& & & 16.88 & 30.70 & 37.15 \\
\hline
\end{tabular}

Table 5.6: The average number of frames with at least one conflict seen by an individual bat in the body coordinate frame for a constant speed maneuver. The top parameter is the maximum sensing range, and the left parameter is the number of neighbors being sensed. A total 1179 frames are used in the analysis.

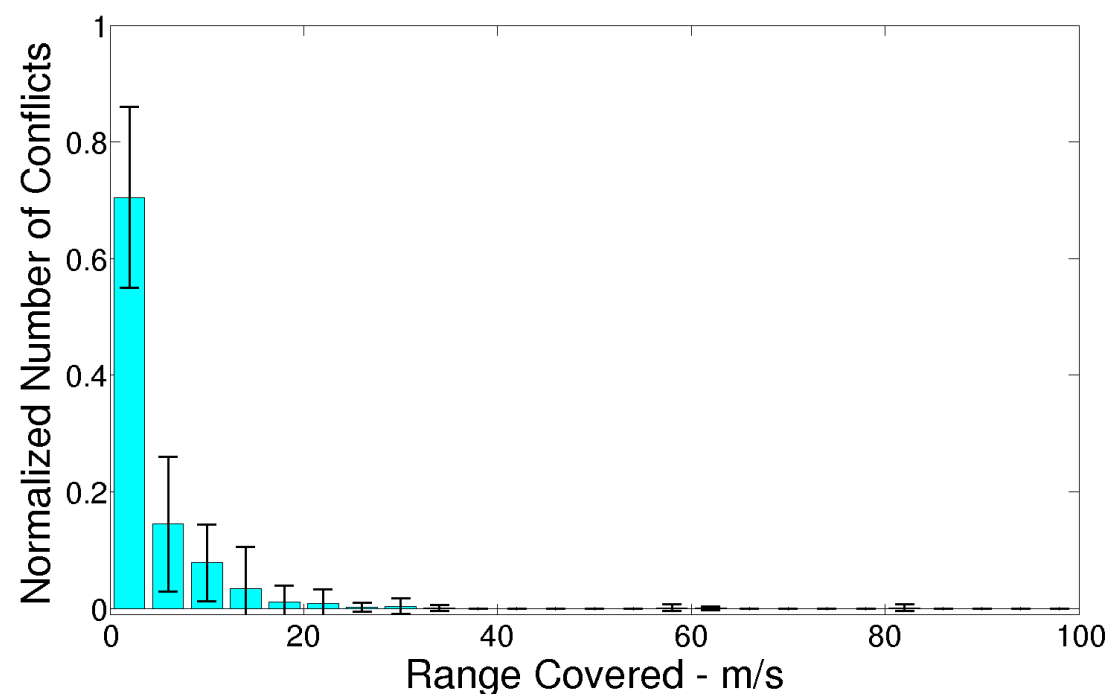

Figure 5.159: The $\Delta V$ distribution for three influential neighbors and $\delta_{\text {attraction }}=$ infinity for the bat data set under the body-fixed coordinate system with the constant speed maneuver. 


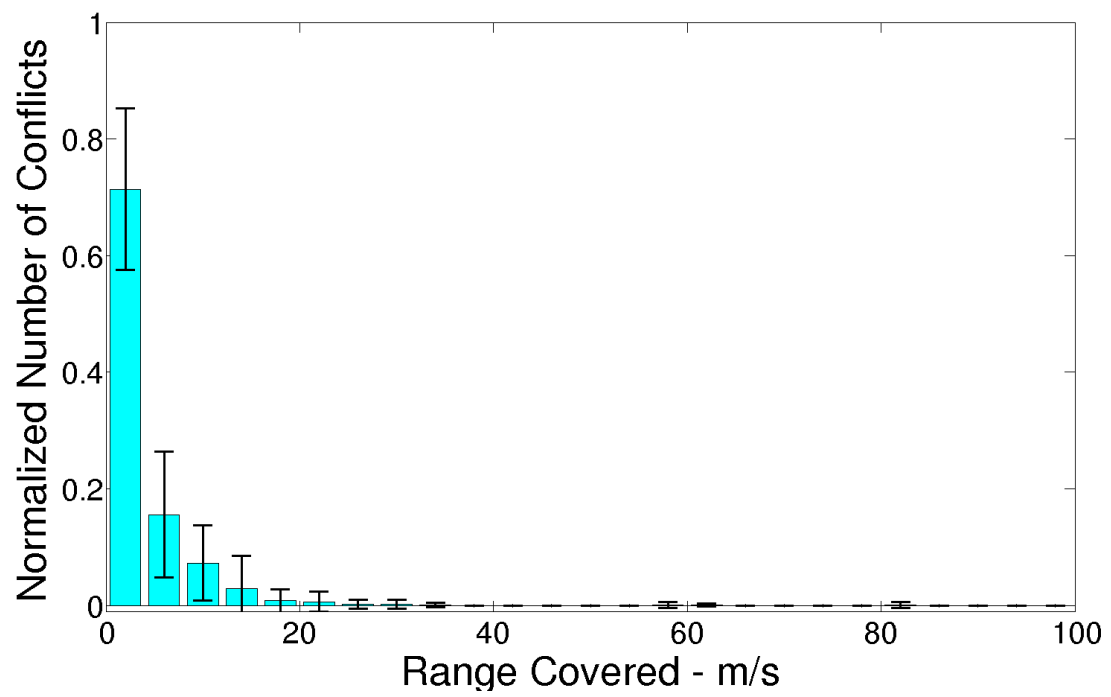

Figure 5.160: The $\Delta V$ distribution for five influential neighbors and $\delta_{\text {attraction }}=$ infinity for the bat data set under the body-fixed coordinate system with the constant speed maneuver.

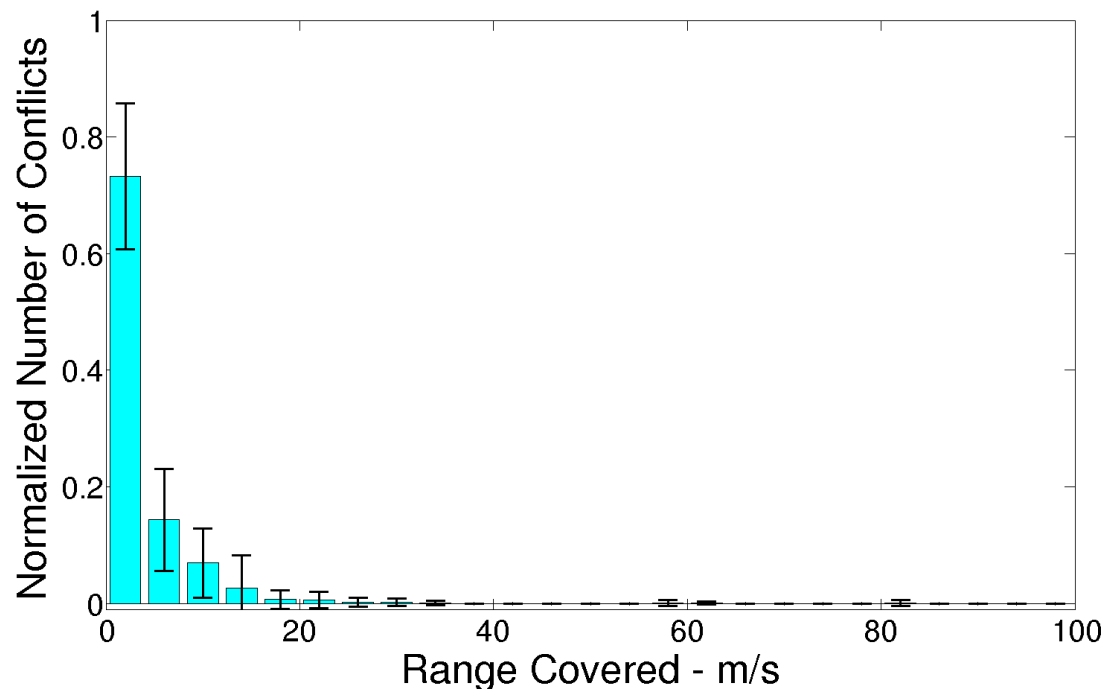

Figure 5.161: The $\Delta V$ distribution for seven influential neighbors and $\delta_{\text {attraction }}=$ infinity for the bat data set under the body-fixed coordinate system with the constant speed maneuver. 


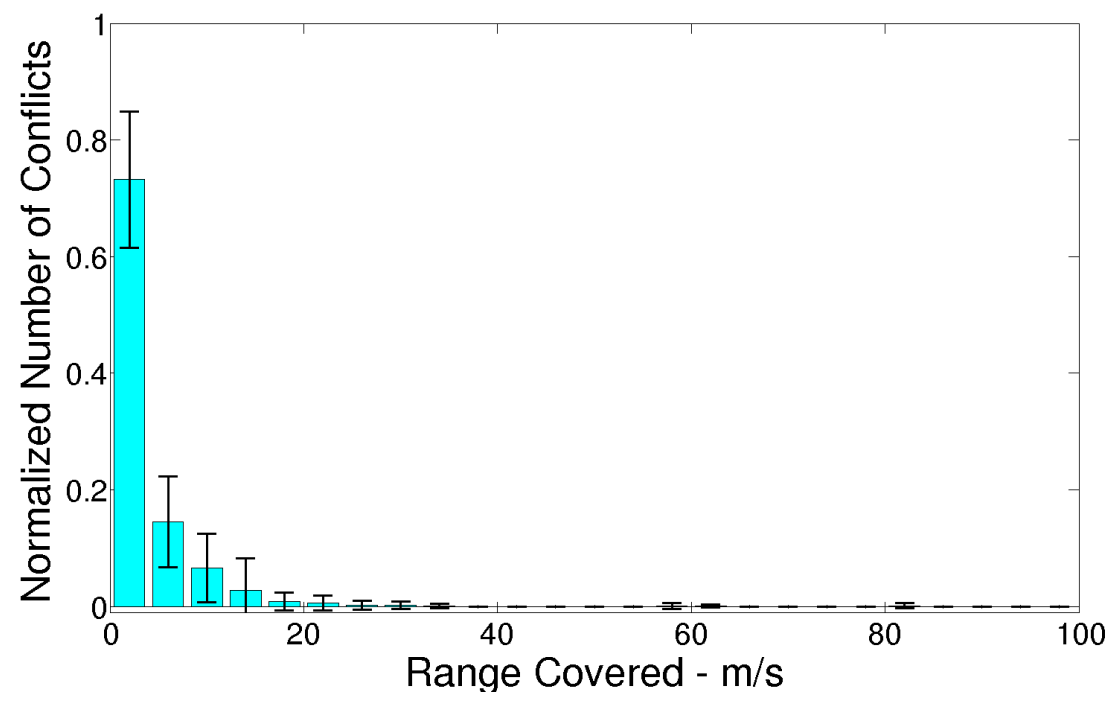

Figure 5.162: The $\Delta V$ distribution for the maximum number of influential neighbors and $\delta_{\text {attraction }}=$ infinity for the bat data set under the body-fixed coordinate system with the constant speed maneuver.

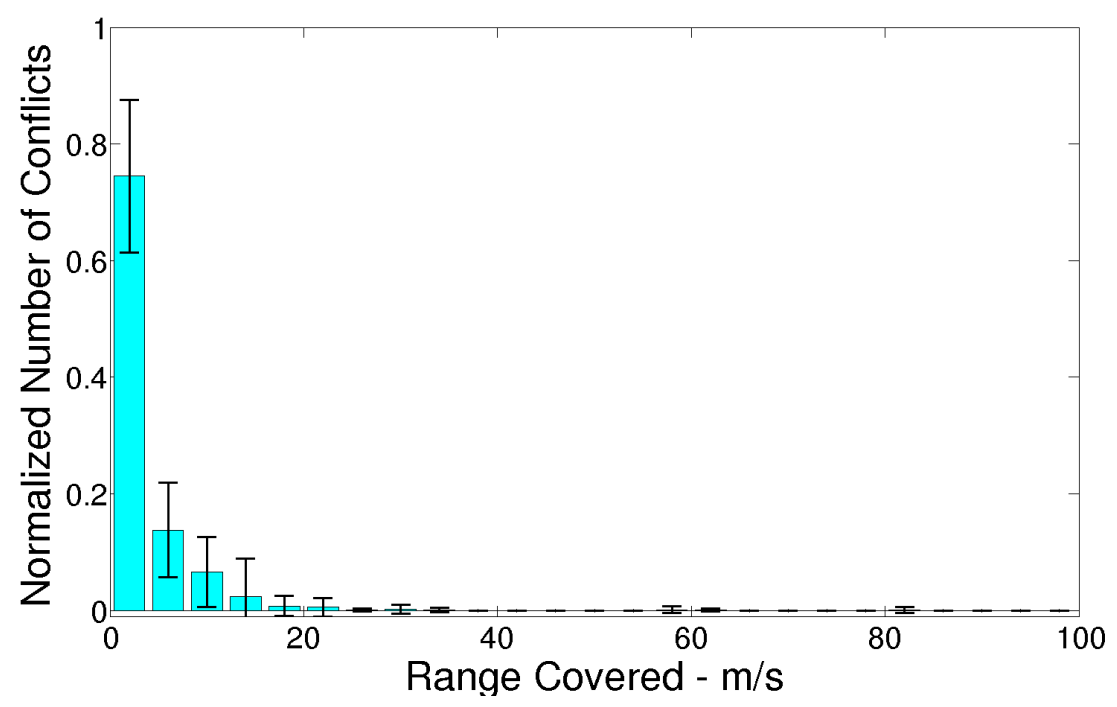

Figure 5.163: The $\Delta V$ distribution for the maximum number of influential neighbors and $\delta_{\text {attraction }}=3 \mathrm{~m}$ for the bat data set under the body-fixed coordinate system with the constant speed maneuver. 


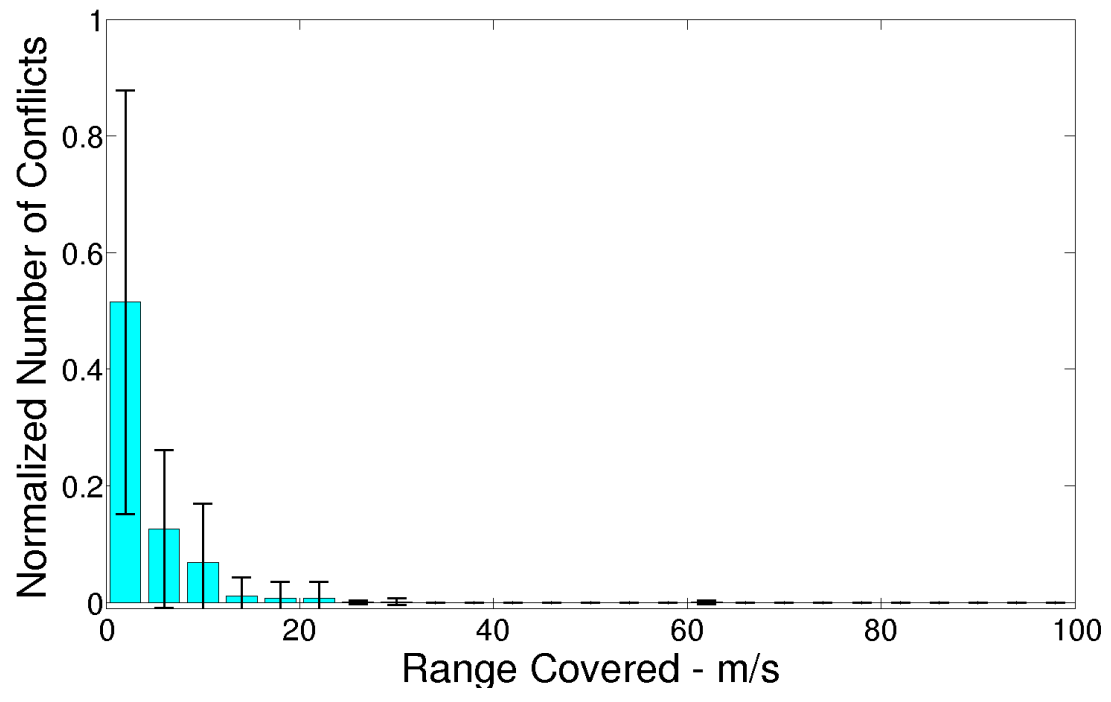

Figure 5.164: The $\Delta V$ distribution for the maximum number of influential neighbors and $\delta_{\text {attraction }}=1 \mathrm{~m}$ for the bat data set under the body-fixed coordinate system with the constant speed maneuver.

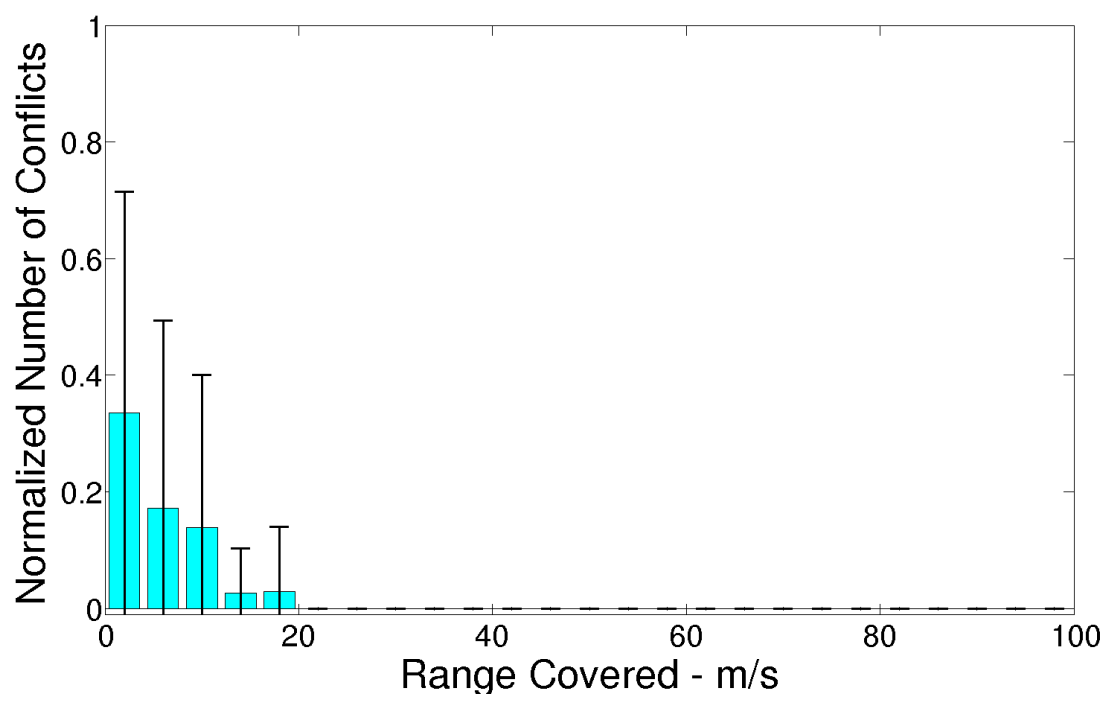

Figure 5.165: The $\Delta V$ distribution for the maximum number of influential neighbors and $\delta_{\text {attraction }}=0.5 \mathrm{~m}$ for the bat data set under the body-fixed coordinate system with the constant speed maneuver. 


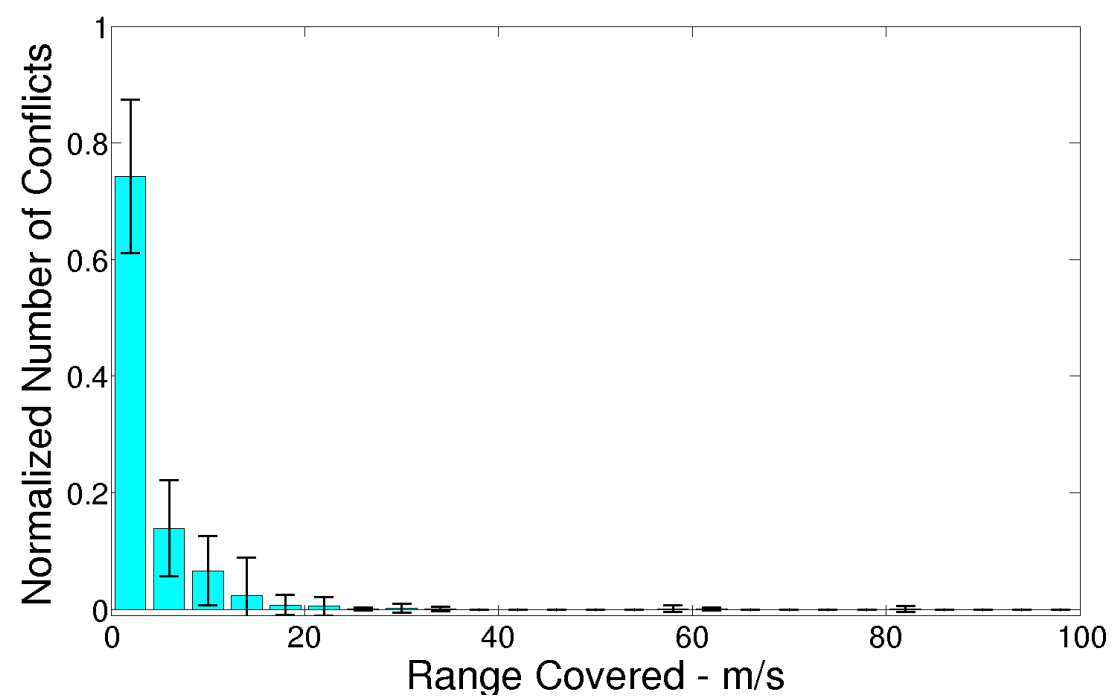

Figure 5.166: The $\Delta V$ distribution for seven influential neighbors and $\delta_{\text {attraction }}=3 \mathrm{~m}$ for the bat data set under the body-fixed coordinate system with the constant speed maneuver.

\begin{tabular}{cc|cccc}
\hline & & \multicolumn{4}{|c}{ Maximum Sensing Range } \\
& & $0.5 \mathrm{~m}$ & $1 \mathrm{~m}$ & $3 \mathrm{~m}$ & infinite \\
\hline \multirow{2}{*}{ Number of } & 5 & $2.27 \mathrm{~s}$ & $2.27 \mathrm{~s}$ & $2.33 \mathrm{~s}$ & $2.33 \mathrm{~s}$ \\
influential Neighbors & 7 & $2.27 \mathrm{~s}$ & $2.27 \mathrm{~s}$ & $2.33 \mathrm{~s}$ & $2.33 \mathrm{~s}$ \\
& All & $2.27 \mathrm{~s}$ & $2.27 \mathrm{~s}$ & $2.33 \mathrm{~s}$ & $2.33 \mathrm{~s}$ \\
& & & $2.27 \mathrm{~s}$ & $2.33 \mathrm{~s}$ & $2.33 \mathrm{~s}$ \\
\hline
\end{tabular}

Table 5.7: The average time, in seconds, in the body coordinate frame scenario with the contstant speed maneuver for an individual bat used to shift its velocity vector closest to that of the optimal. The top parameter is the maximum sensing range in meters, and the left parameter is the number of neighbors being sensed. 


\begin{tabular}{cc|cccc}
\hline & & \multicolumn{4}{|c}{ Maximum Sensing Range } \\
& & $0.5 \mathrm{~m}$ & $1 \mathrm{~m}$ & $3 \mathrm{~m}$ & infinite \\
\hline \multirow{2}{*}{ Number of } & 5 & 9.33 & 17.00 & 25.25 & 26.20 \\
Influential Neighbors & 7 & 9.33 & 17.56 & 28.85 & 31.20 \\
& All & 9.33 & 17.69 & 31.35 & 34.95 \\
& & 17.69 & 31.55 & 38.10 \\
\hline
\end{tabular}

Table 5.8: The average number of frames with at least one conflict seen by an individual bat in the body coordinate frame for a variable speed maneuver. The top parameter is the maximum sensing range, and the left parameter is the number of neighbors being sensed. A total 1179 frames are used in the analysis.

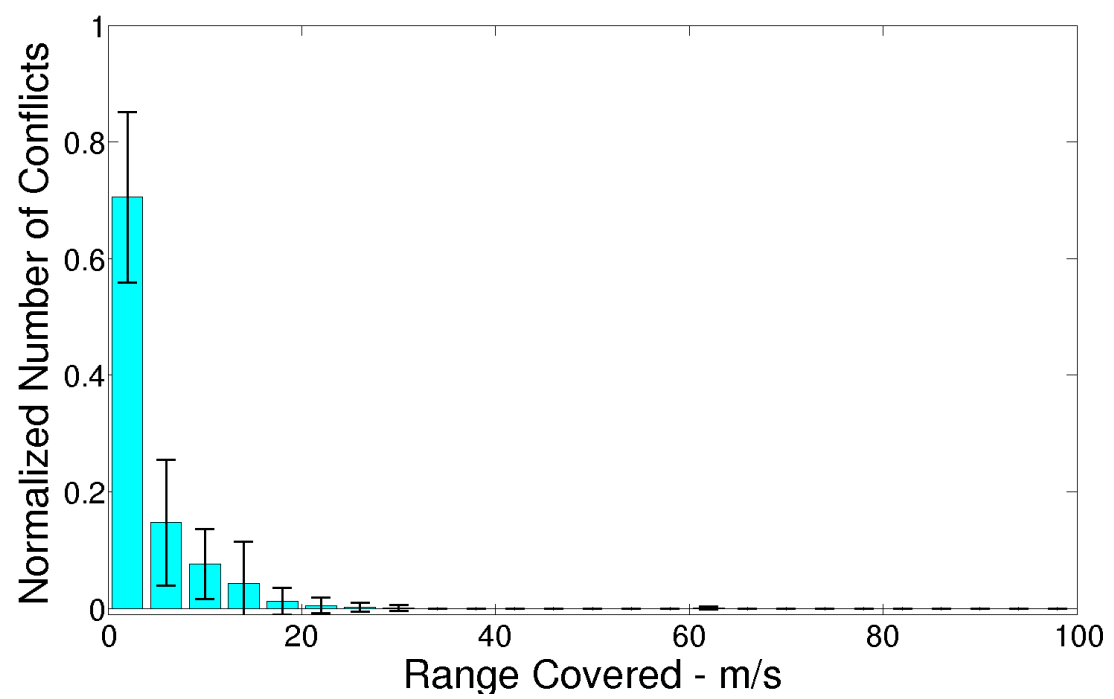

Figure 5.167: The $\Delta V$ distribution for three influential neighbors and $\delta_{\text {attraction }}=$ infinity for the bat data set under the body-fixed coordinate system with the variable speed maneuver. 


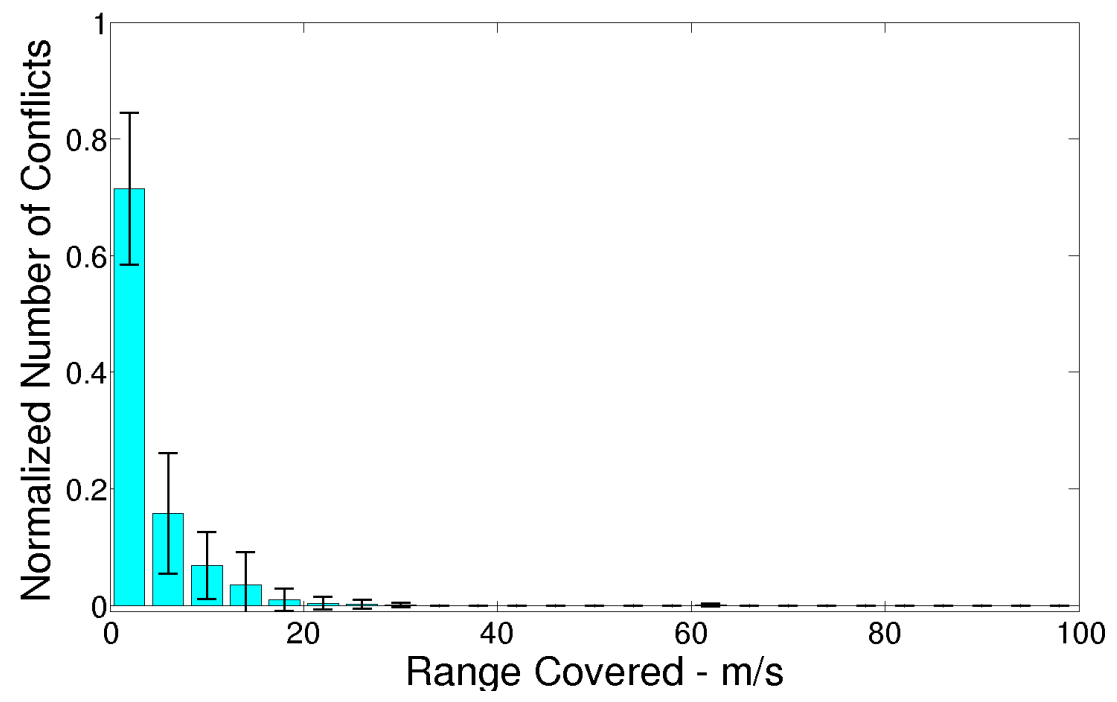

Figure 5.168: The $\Delta V$ distribution for five influential neighbors and $\delta_{\text {attraction }}=$ infinity for the bat data set under the body-fixed coordinate system with the variable speed maneuver.

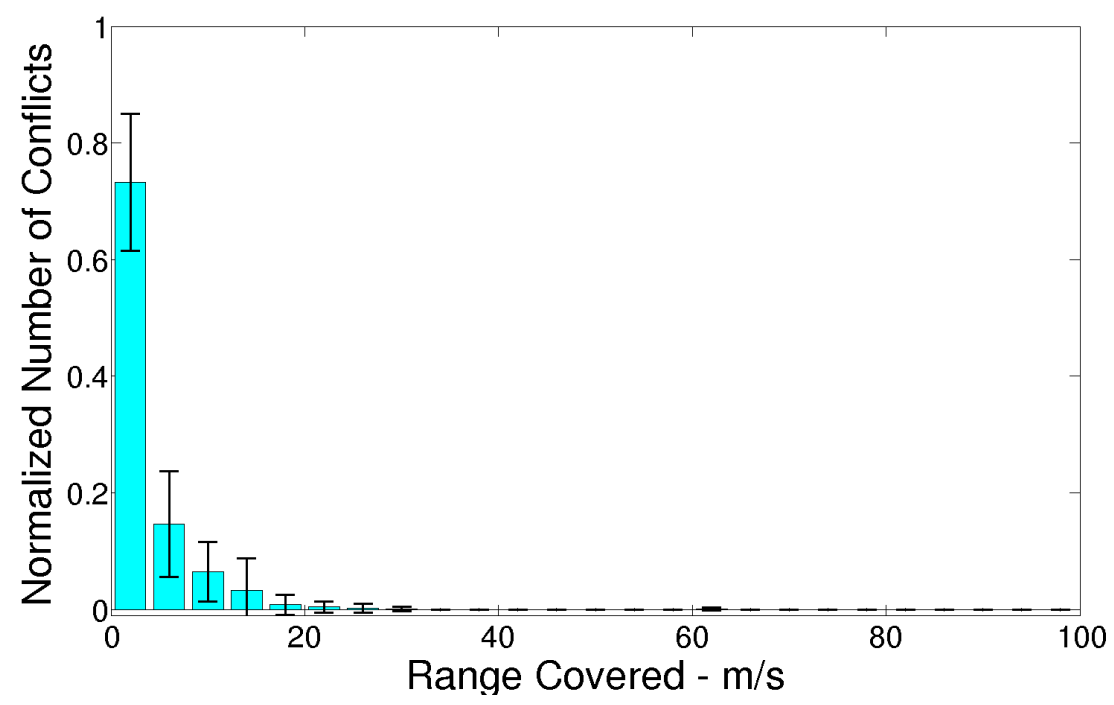

Figure 5.169: The $\Delta V$ distribution for seven influential neighbors and $\delta_{\text {attraction }}=$ infinity for the bat data set under the body-fixed coordinate system with the variable speed maneuver. 


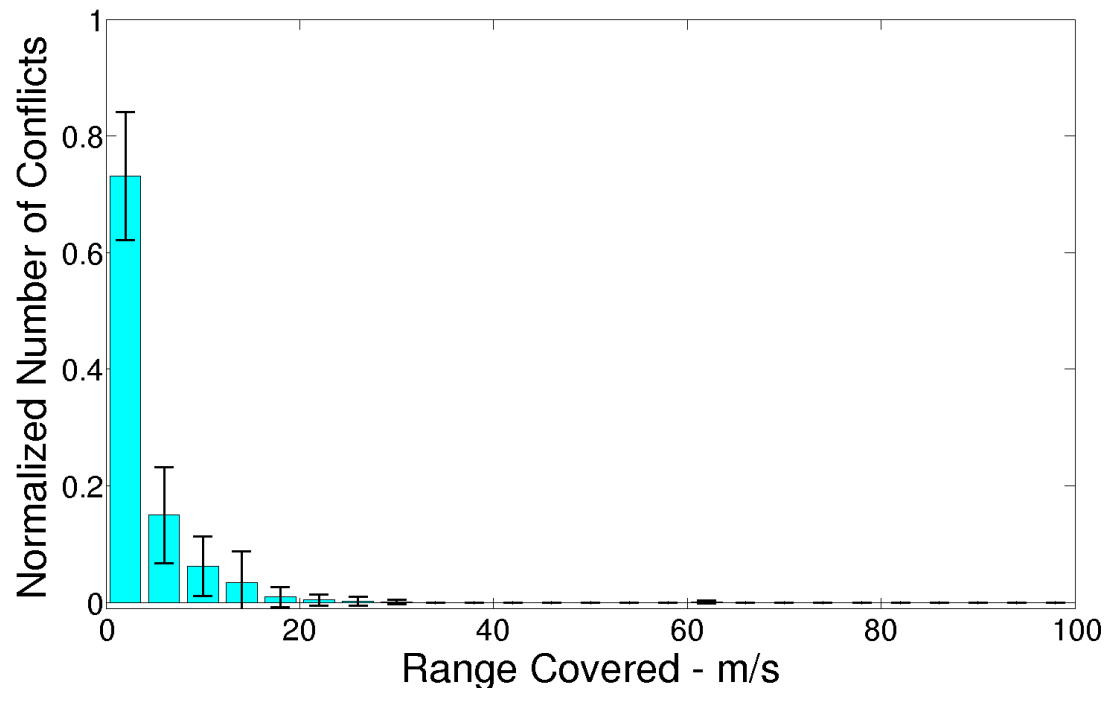

Figure 5.170: The $\Delta V$ distribution for the maximum number of influential neighbors and $\delta_{\text {attraction }}=$ infinity for the bat data set under the body-fixed coordinate system with the variable speed maneuver.

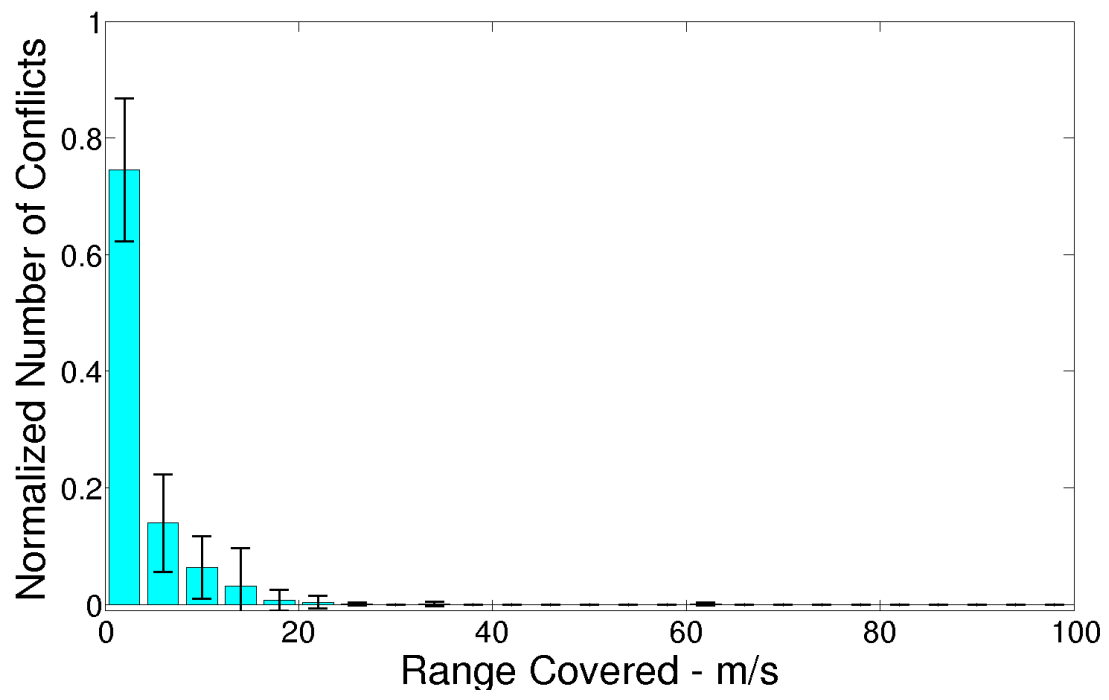

Figure 5.171: The $\Delta V$ distribution for the maximum number of influential neighbors and $\delta_{\text {attraction }}=3 \mathrm{~m}$ for the bat data set under the body-fixed coordinate system with the variable speed maneuver. 


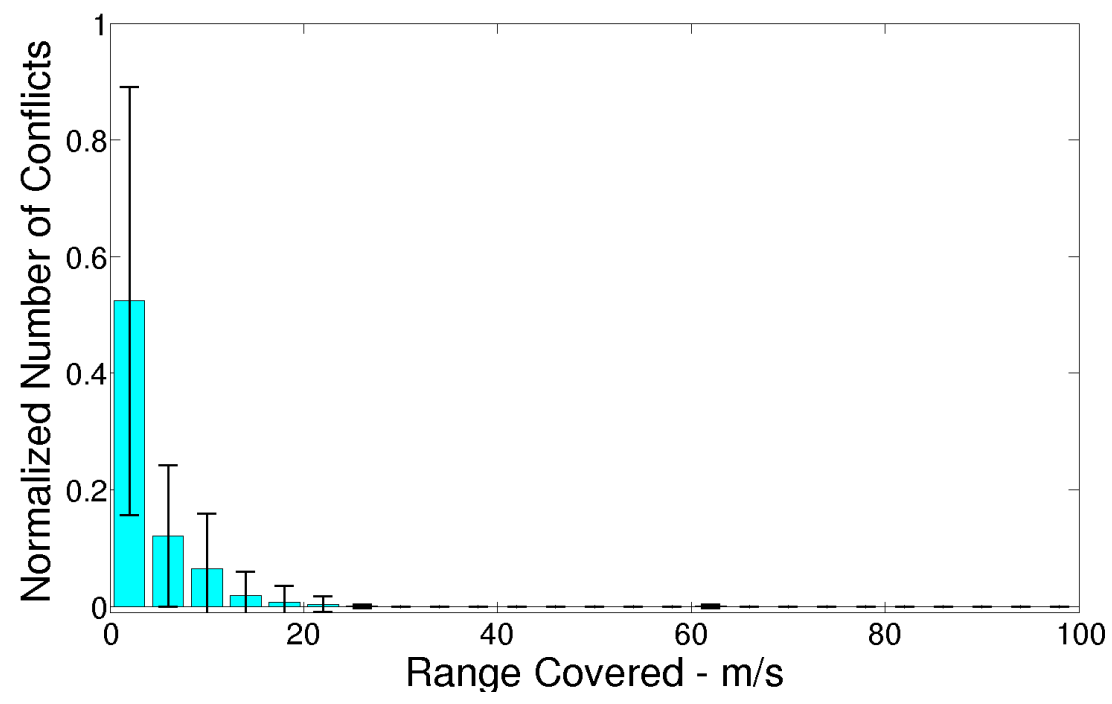

Figure 5.172: The $\Delta V$ distribution for the maximum number of influential neighbors and $\delta_{\text {attraction }}=1 \mathrm{~m}$ for the bat data set under the body-fixed coordinate system with the variable speed maneuver.

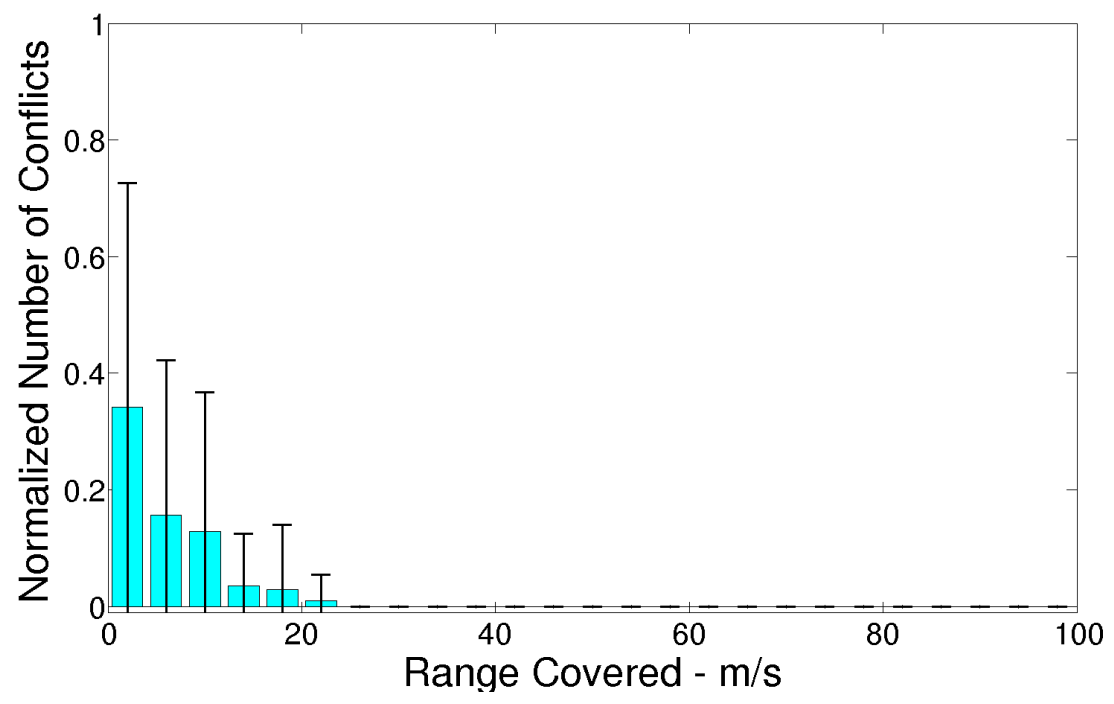

Figure 5.173: The $\Delta V$ distribution for the maximum number of influential neighbors and $\delta_{\text {attraction }}=0.5 \mathrm{~m}$ for the bat data set under the body-fixed coordinate system with the variable speed maneuver. 


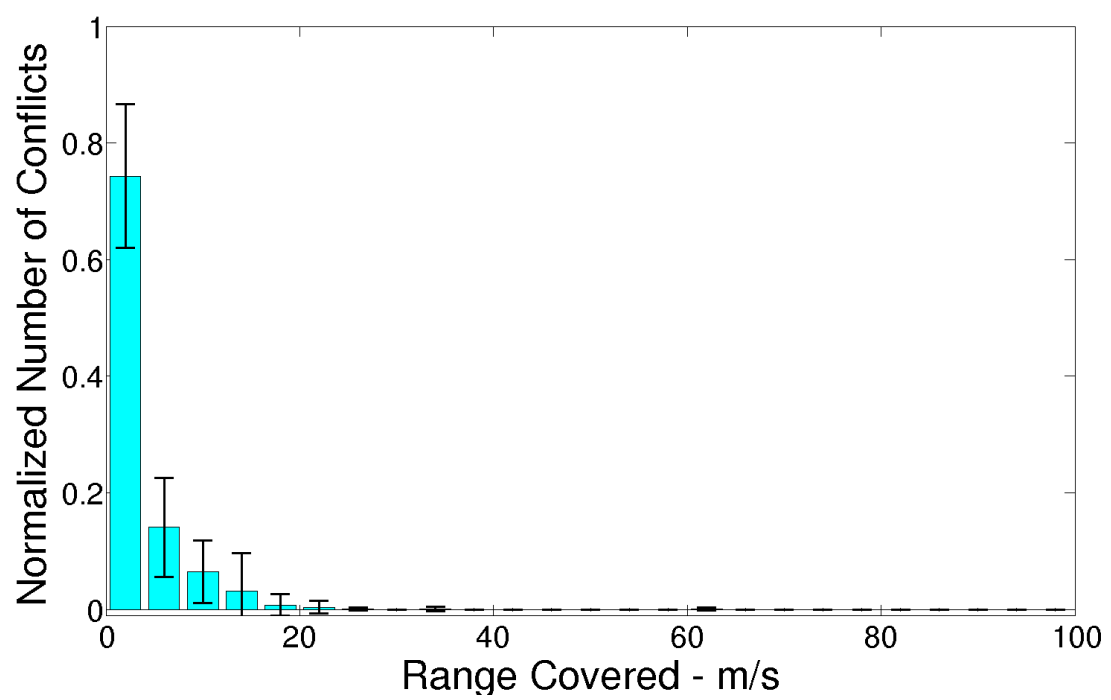

Figure 5.174: The $\Delta V$ distribution for seven influential neighbors and $\delta_{\text {attraction }}=3 \mathrm{~m}$ for the bat data set under the body-fixed coordinate system with the variable speed maneuver.

\begin{tabular}{cl|cccc}
\hline & & \multicolumn{4}{|c}{ Maximum Sensing Range } \\
& & $0.5 \mathrm{~m}$ & $1 \mathrm{~m}$ & $3 \mathrm{~m}$ & infinite \\
\hline \multirow{3}{*}{ Number of } & 5 & $2.27 \mathrm{~s}$ & $2.27 \mathrm{~s}$ & $2.33 \mathrm{~s}$ & $2.33 \mathrm{~s}$ \\
Influential Neighbors & 7 & $2.27 \mathrm{~s}$ & $2.27 \mathrm{~s}$ & $2.33 \mathrm{~s}$ & $2.33 \mathrm{~s}$ \\
& All & $2.27 \mathrm{~s}$ & $2.27 \mathrm{~s}$ & $2.33 \mathrm{~s}$ & $2.33 \mathrm{~s}$ \\
& & $2.27 \mathrm{~s}$ & $2.33 \mathrm{~s}$ & $2.33 \mathrm{~s}$ \\
\hline
\end{tabular}

Table 5.9: The average time, in seconds, in the body coordinate frame scenario with the variable speed maneuver for an individual bat used to shift its velocity vector closest to that of the optimal. The top parameter is the maximum sensing range in meters, and the left parameter is the number of neighbors being sensed. 
Chapter 6

\section{CONCLUSION AND FUTURE WORK}

This thesis is a comparison of an analytical deconfliction algorithm, based on collision cones, to a spectrum of biological flight data. Three biological species were used for this comparison: giant Danios, barn swallows, and cave bats. The collision avoidance algorithm comparison analyzed with respect to two different coordinate systems, inertia and bodyfixed and the parameters of number of influential neighbors ad the maximum sensing range based on a zonal model..

The best correlation between the giant Danio data and the algorithm was found for the global coordinate variable speed maneuver, with the verage $\Delta V$ less than $4 \mathrm{~cm} / \mathrm{s}$ an average of $70 \%$ of the time. The giant Danio's perception of their underwater environment may be different than expected, causing the body-fixed coordinate system to produce lower correlated results compared to the global coordinate system. The Mid segment consistently yielded the lowest correlations of all the segments. This lower correlation could be a result of the fish actively searching for food and caring less about optimally avoiding collisions with their neighbors.

The analysis of the barn swallow data showed that the variable speed maneuver yielded slightly better correlations than did the constant speed maneuver. The variable speed maneuver was what was expected since the swallows can change thier speed during flight. No appreciable change in the correlations between swallow data and the algorithm could be found when using the global coordinate system versus the body-fixed coordinate system. Little to no difference in how well correlated the data and algorithm were, when using data sets with or without static obstacles, suggests that swallows were avoiding static obstacles in a similar way to how they avoid fellow swallows. The swallow data produced the highest correlated results when the number of influential neighbors was limited, which is congruent with the results in [1]. To fully analyze the barn swallow data, a three-dimensional algorithm 
is needed, especially when obstacles such as rooflines are present in the data.

Analysis of the bat data showed that the data and algorithm were more highly correlated when the algorithm used the body-fixed coordinate system instead of the global coordinate system. The correlation between the bat data and the algoithm did not change much when using the constant speed maneuver versus the variable speed maneuver. Unlike the swallow data, the bat data correlation results varied more when changing the maximum sensing range as opposed to changing the number of influencial neighbors.

\subsection{Future Work}

In the future, a three-dimensional algorithm should be created to fully analyze the flying species. Also, another coordinate system could be developed in hopes of better representing how the different species view their surroundings. Incorporating the head pose rather than the body velocity direction, particularly for bats and swallows, might be important. Comparing the data against other deconfliction algorithms, i.e. potential functions, could yield results that are more highly correlated than the collision cone approach used in this thesis. Scenarios may exist, such as during the food stimulus, where another, more basic, deconfliction algorithm would be more highly correlated to the data. When food is a factor, animals may not care about deconfliction as long as they get the most food. More data sets for all species would be helpful in confirming the results presented here. The majority of the data sets presented in this thesis are unique with respect to the number of animals present or the type of obstacles. A more complete analysis of the response time of each species should be done in the near future and compared to other studies of reaction time. To make the algorithm more realistic, limiting the sensing in areas to create blind spots may be useful. 


\section{BIBLIOGRAPHY}

[1] M. Ballerini, N. Cabibbo, R. Candelier, A. Cavagna, E. Cisbani, I. Giardina, V. Lecomte, A. Orlandi, G. Parisi, A. Procaccini, et al. Interaction ruling animal collective behavior depends on topological rather than metric distance: Evidence from a field study. Proceedings of the National Academy of Sciences, 105(4):1232, 2008.

[2] J. Buhl, D.J.T. Sumpter, I.D. Couzin, J.J. Hale, E. Despland, E.R. Miller, and S.J. Simpson. From disorder to order in marching locusts. Science, 312(5778):1402-1406, 2006.

[3] S. Camazine. Self-organization in biological systems. Princeton University Press, 2003.

[4] D.S. Cambuí and A. Rosas. Density induced transition in a school of fish. Physica A: Statistical Mechanics and its Applications, 391:3908-3914, 2012.

[5] A. Chakravarthy and D. Ghose. Obstacle avoidance in a dynamic environment: A collision cone approach. IEEE Transactions on Systems, Man and Cybernetics, Part A: Systems and Humans, 28(5):562-574, 1998.

[6] I.D. Couzin and N.R. Franks. Self-organized lane formation and optimized traffic flow in army ants. Proceedings of the Royal Society of London, Series B: Biological Sciences, 270(1511):139-146, 2003.

[7] I.D. Couzin, C.C. Ioannou, G. Demirel, T. Gross, C.J. Torney, A. Hartnett, L. Conradt, S.A. Levin, and N.E. Leonard. Uninformed individuals promote democratic consensus in animal groups. Science, 334(6062):1578-1580, 2011.

[8] M.S. Eby and W.E. Kelly III. Free flight separation assurance using distributed algorithms. In IEEE Aerospace Conference Proceedings, volume 2, pages 429-441. IEEE, 1999 .

[9] E. Frazzoli, Z.H. Mao, J.H. Oh, and E. Feron. Resolution of conflicts involving many aircraft via semidefinite programming. AIAA Journal of Guidance, Control, and Dynamics, 24:79-86, 2001.

[10] G. Grégoire, H. Chaté, and Y. Tu. Moving and staying together without a leader. Physica D: Nonlinear Phenomena, 181(3-4):157-170, 2003.

[11] D. Grünbaum. Tracker 3d movement analysis software. User's guide, 2003. 
[12] R. Hartley and A. Zisserman. Multiple view geometry in computer vision. Cambridge University Press, 2003.

[13] Bruce Hayward and Russell Davis. Flight speeds in western bats. Journal of Mammalogy, 45(2):236-242, 1964.

[14] T.L. Hedrick. Software techniques for two-and three-dimensional kinematic measurements of biological and biomimetic systems. Bioinspiration \& Biomimetics, 3(3):1-6, 2008 .

[15] J.E. Herbert-Read, A. Perna, R.P. Mann, T.M. Schaerf, D.J.T. Sumpter, and A.J.W. Ward. Inferring the rules of interaction of shoaling fish. Proceedings of the National Academy of Sciences, 108(46):18726-18731, 2011.

[16] G.M. Hoffmann and C.J. Tomlin. Decentralized cooperative collision avoidance for acceleration constrained vehicles. In 47 th IEEE Conference on Decision and Control, pages 4357-4363. IEEE, 2008.

[17] I. Hwang, J. Kim, and C. Tomlin. Protocol-based conflict resolution for air traffic control. Air Traffic Control Quarterly, 15(1):1-34, 2007.

[18] Y. Inada and K. Kawachi. Order and flexibility in the motion of fish schools. Journal of Theoretical Biology, 214(3):371-387, 2002.

[19] J.K. Kuchar and L.C. Yang. A review of conflict detection and resolution modeling methods. IEEE Transactions on Intelligent Transportation Systems, 1(4):179-189, 2000 .

[20] E. Lalish. Distributed reactive collision avoidance for multivehicle systems. PhD thesis, University of Washington, 2009.

[21] E. Lalish, K.A. Morgansen, and T. Tsukamaki. Decentralized reactive collision avoidance for multiple unicycle-type vehicles. In American Control Conference, 2008, pages 5055-5061. IEEE, 2008.

[22] K.C. Mushambi. Application of modern filtering techniques for 3D localisation in biological and robotic systems. Master's thesis, University of Washington, 2006.

[23] L. Pallottino, V.G. Scordio, A. Bicchi, and E. Frazzoli. Decentralized cooperative policy for conflict resolution in multivehicle systems. IEEE Transactions on Robotics, 23(6):1170-1183, 2007.

[24] J.K. Parrish, S.V. Viscido, and D. Grünbaum. Self-organized fish schools: an examination of emergent properties. The Biological Bulletin, 202(3):296-305, 2002. 
[25] A. Pongpunwattana and R. Rysdyk. Real-time planning for multiple autonomous vehicles in dynamic uncertain environments. Journal of Aerospace Computing, Information, and Communication, 1(12):580-604, 2004.

[26] A.R. Rahmani. Networked dynamic systems: Theory and applications. PhD thesis, University of Washington, 2008.

[27] A. Stanley. Flight path deconfliction of autonomous uavs. AIAA Infotech@ Aerospace, 2005 .

[28] S.V. Viscido, J.K. Parrish, and D. Grünbaum. Individual behavior and emergent properties of fish schools: A comparison of observation and theory. Marine Ecology Progress Series, 273:239-249, 2004.

[29] S.V. Viscido, J.K. Parrish, and D. Grünbaum. The effect of population size and number of influential neighbors on the emergent properties of fish schools. Ecological Modelling, 183(2):347-363, 2005.

[30] S.V. Viscido, J.K. Parrish, and D. Grünbaum. Factors influencing the structure and maintenance of fish schools. Ecological modelling, 206(1):153-165, 2007.

[31] S. Wang and H. Schaub. Spacecraft collision avoidance using coulomb forces with separation distance and rate feedback. Journal of Guidance, Control, and Dynamics, $31(3): 740-750,2008$.

[32] Y.T. Yang, A. Quitmeyer, B. Hrolenok, H. Shang, D.B. Nguyen, T. Balch, T. Medina, C. Sherer, M. Hybinette, and GA Athens. Ant hunt: Towards a validated model of live ant hunting behavior. In Twenty-Fifth International FLAIRS Conference, 2012. 